\title{
Dynamic Building Load Control to Facilitate High Penetration of Solar Photovoltaic Generation Final Technical Report
}

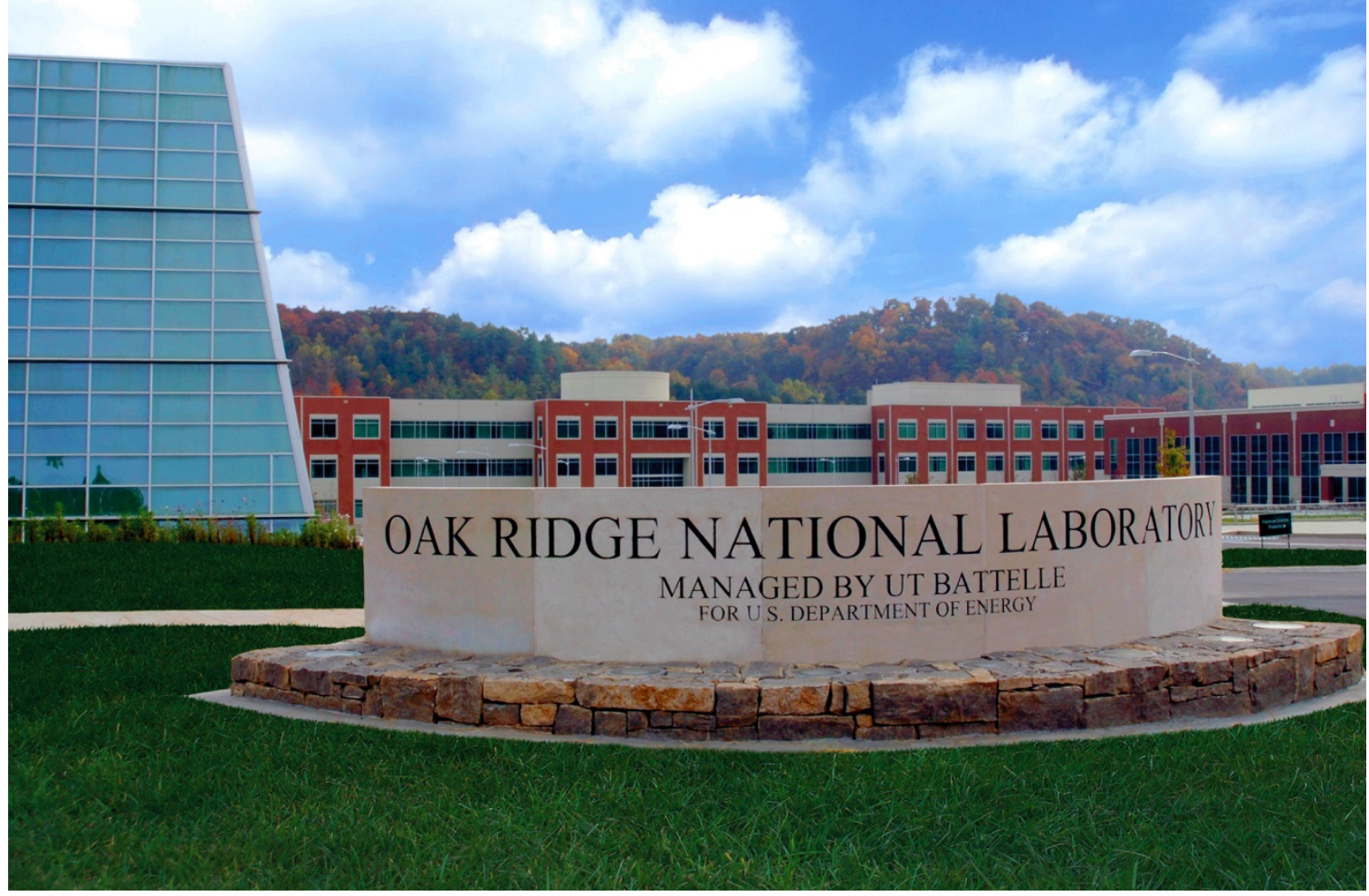

Teja Kuruganti Mohammed Olama Jin Dong Yaosuo Xue Christopher Winstead James Nutaro Seddik Djouadi Linquan Bai Godfried Augenbroe Justin Hill Approved for public release. Distribution is unlimited.

September 2021 


\title{
DOCUMENT AVAILABILITY
}

Reports produced after January 1, 1996, are generally available free via US Department of Energy (DOE) SciTech Connect.

Website www.osti.gov

Reports produced before January 1, 1996, may be purchased by members of the public from the following source:

\author{
National Technical Information Service \\ 5285 Port Royal Road \\ Springfield, VA 22161 \\ Telephone 703-605-6000 (1-800-553-6847) \\ TDD 703-487-4639 \\ Fax 703-605-6900 \\ E-mail info@ntis.gov \\ Website http://classic.ntis.gov/
}

Reports are available to DOE employees, DOE contractors, Energy Technology Data Exchange representatives, and International Nuclear Information System representatives from the following source:

Office of Scientific and Technical Information

PO Box 62

Oak Ridge, TN 37831

Telephone 865-576-8401

Fax 865-576-5728

E-mail reports@osti.gov

Website https://www.osti.gov/

This report was prepared as an account of work sponsored by an agency of the United States Government. Neither the United States Government nor any agency thereof, nor any of their employees, makes any warranty, express or implied, or assumes any legal liability or responsibility for the accuracy, completeness, or usefulness of any information, apparatus, product, or process disclosed, or represents that its use would not infringe privately owned rights. Reference herein to any specific commercial product, process, or service by trade name, trademark, manufacturer, or otherwise, does not necessarily constitute or imply its endorsement, recommendation, or favoring by the United States Government or any agency thereof. The views and opinions of authors expressed herein do not necessarily state or reflect those of the United States Government or any agency thereof. 
Computational Sciences and Engineering Division

\title{
DYNAMIC BUILDING LOAD CONTROL TO FACILITATE HIGH PENETRATION OF SOLAR PHOTOVOLTAIC GENERATION FINAL TECHNICAL REPORT
}

\author{
Teja Kuruganti \\ Mohammed Olama \\ Jin Dong \\ Yaosuo Xue \\ Christopher Winstead \\ James Nutaro \\ Seddik Djouadi \\ Linquan Bai \\ Godfried Augenbroe \\ Justin Hill
}

September 2021

\author{
Prepared by \\ OAK RIDGE NATIONAL LABORATORY \\ Oak Ridge, TN 37831-6283 \\ managed by \\ UT-BATTELLE LLC \\ for the \\ US DEPARTMENT OF ENERGY \\ under contract DE-AC05-00OR22725
}





\section{CONTENTS}

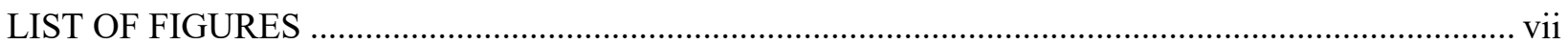

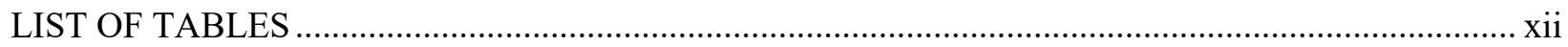

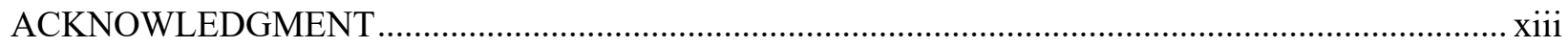

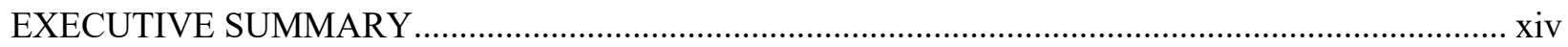

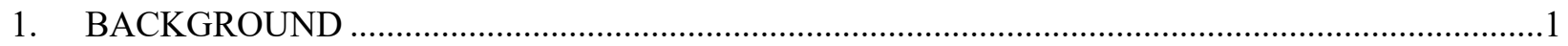

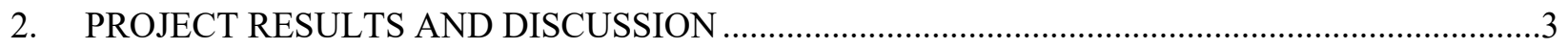

2.1 SPECTRAL ANALYSIS OF SOLAR PV POWER OUTPUT ..................................................

2.2 FREQUENCY-DOMAIN ANALYSES FOR THE VARIOUS BUILDING TCLS...................7

2.3 DISTRIBUTION-LEVEL PV INTEGRATION AND PENETRATION ANALYSIS ..............8

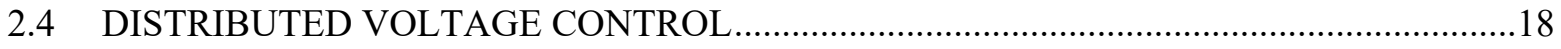

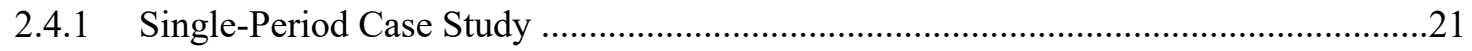

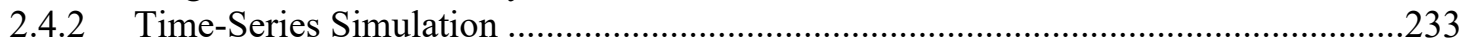

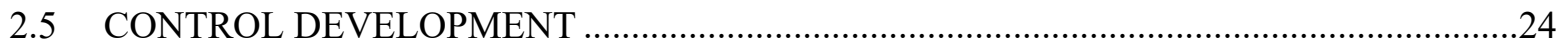

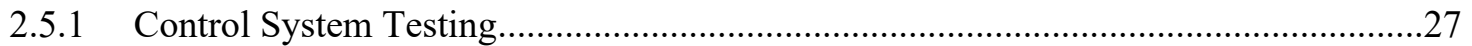

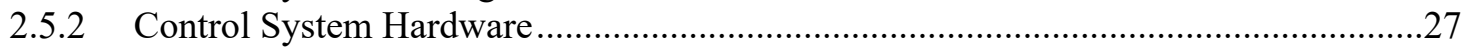

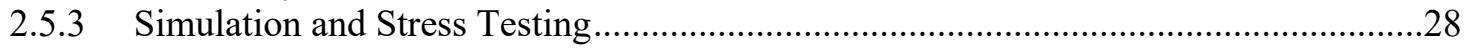

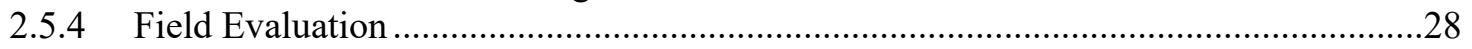

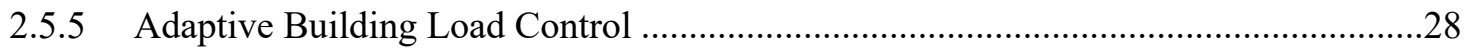

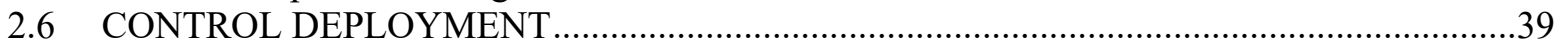

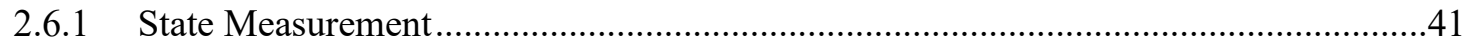

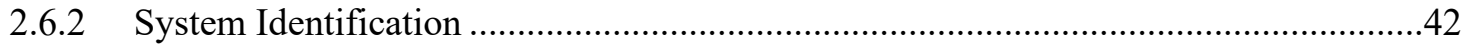

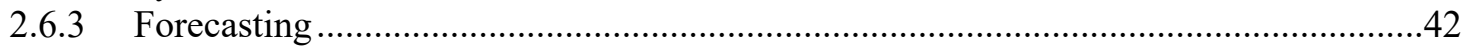

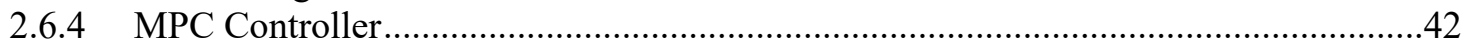

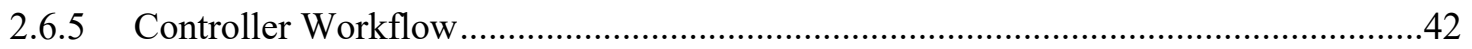

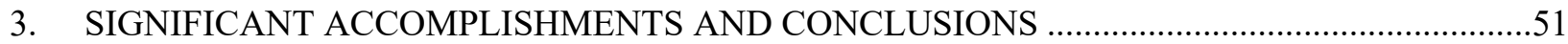

4. INVENTIONS, PATENTS, PUBLICATIONS, AND OTHER RESULTS...........................................

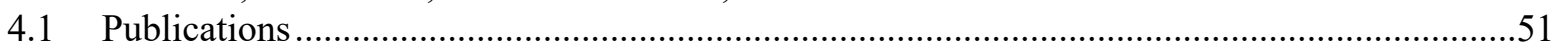

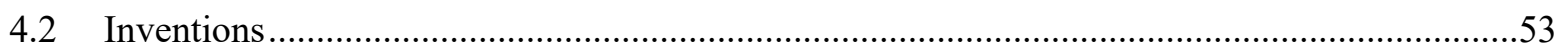

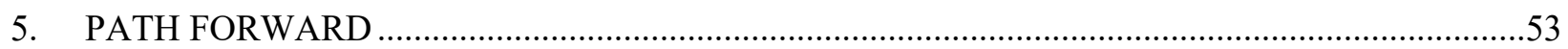

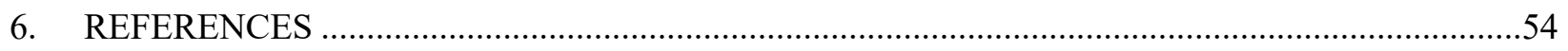

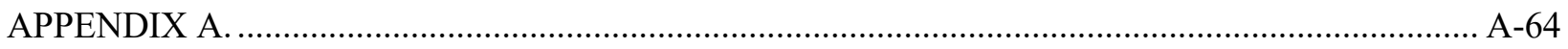

A.1 BACKGROUND ON DISTRIBUTION SYSTEM VOLTAGE CONTROL.................... A-64

A.1.1 Hardware Components of Solar PV Systems.................................................... A-64

A.1.2 Power Electronics Architectures for Solar PV Grid Interconnection ..................... A-64

A.1.3 Distribution System Voltage Control...................................................................... A-64

A.2 COORDINATION OF BUILDING HVAC LOAD CONTROL WITH SMART PV

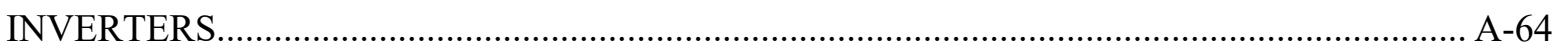

A.2.1 Control Integration at ADMS Level .......................................................... A -64

A.2.2 Control Coordination at Local Node........................................................... A-64

A.2.3 The Need for Distributed Control Approach .................................................. A-64

A.2.4 Concept of a New DVC ............................................................................ A-64

A.3 IEEE 13-NODE TEST FEEDER DISTRIBUTION-LEVEL PV PENETRATION

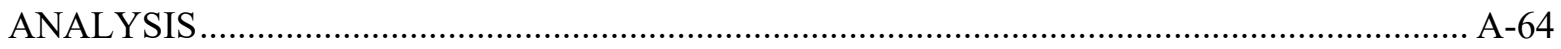

A.3.1 Annual Load and Solar Power Profiles ............................................................ A-64

A.3.2 Solar Power Production at Different Penetration Levels ....................................... A-64

A.3.3 Impact on Steady-State Voltage Profile .............................................................. A-64

A.3.4 Impact on Substation Voltage, PF, and Capacitor Voltage..................................... A-64

A.3.5 Impact on Voltage Regulator LTC Operation.................................................... A-64 
A.3.6 Smart PV Inverter with Voltage Regulation Function......................................... A-64

A.3.7 Dedicated Volt/Var Compensation Devices ...................................................... A-64

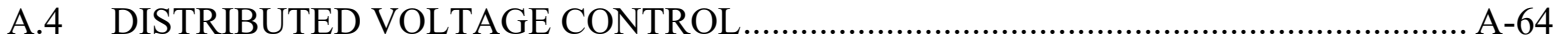

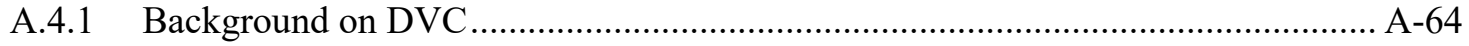

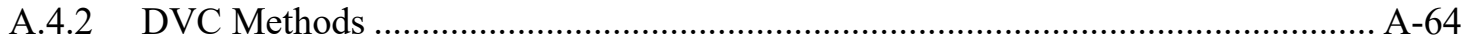

A.4.3 Adaptive and/or Feedback Voltage Control........................................................ A-64

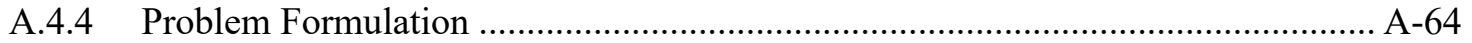

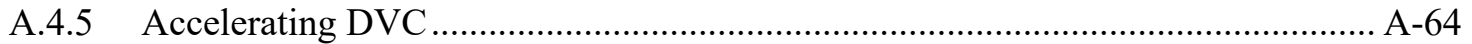

A.5 BUILDING MODEL PARAMETERS ESTIMATION …......................................... A-64

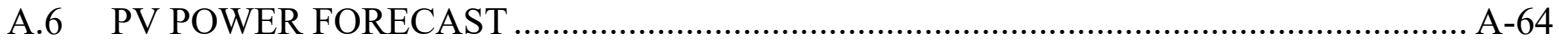

A.7 ADAPTIVE BUILDING LOAD CONTROL (MFC) …................................................ A-64

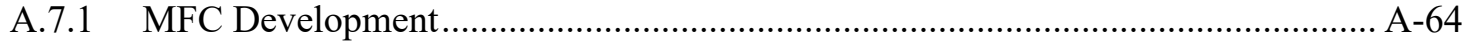

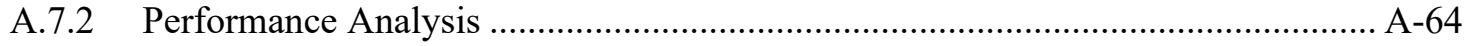

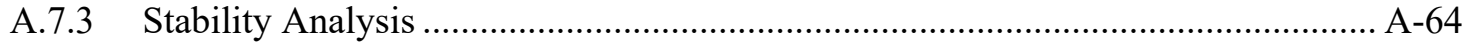

A.7.4 Generalization of MFC to Different Types of Loads .......................................... A-64

A.8 DETAILS ON CONTROLLER DEPLOYMENT _...................................................... A-64

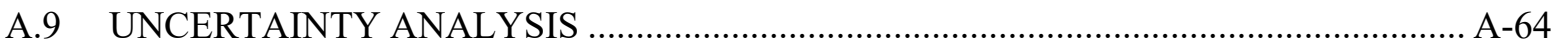

A.9.1 Uncertainty Analysis for Building Power Consumption .................................... A-64

A.9.2 The Analysis of Zonal Controllability in HVAC System Power Interruption........ A-64

A.9.3 Uncertainty Quantification of RC Model.............................................................. A-64

A.9.4 Integrating the Building Energy Model Inside the Overall System Architecture ... A-64 


\section{LIST OF FIGURES}

Figure 1. The proposed PV power filtering approach.

Figure 2. (a) Filtering process of input PV power; (b) PV power profile for one summer day in

2015 and its corresponding low-, medium-, and high-frequency content.

Figure 3. (a) The time-domain solar PV power profiles for eight summer days collected from a 13

$\mathrm{kW}$ solar PV panel showing fast and large fluctuations of PV outputs and (b) their

corresponding frequency-domain power profiles.

Figure 4. The PV power output time-frequency PSD for one summer day, illustrating how the PV power spectrum of frequencies varies with time.

Figure 5. Boxplots illustrating the 2015 (a) annual and (b) seasonal statistics for the spectrum of PV power outputs

Figure 6. (a) Charging/discharging power levels and (b) SOC of the battery.

Figure 7. Boxplots illustrating the annual statistics for the spectrum of (a) on/off HVAC, (b) WH, and (c) refrigeration power consumptions and (d) compared with the one of $\mathrm{PV}$.........................8

Figure 8. Comparison of duration curves for HVAC, WH, and PV powers. .............................................

Figure 9. Circuit WEBB DS 28266 separated using OpenDSS Tools....................................................

Figure 10. Circuit WEBB DS 28260 separated using OpenDSS Tools.................................................

Figure 11. WEBB feeder daily load profile (measurements on July 2, 2016) ........................................10

Figure 12. Distribution voltage regulation problems due to solar PV generation. ....................................10

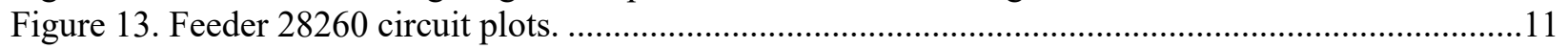

Figure 14. Feeder 28260 snapshot voltage profiles (spider plots). ......................................................12

Figure 15. Power and voltage dynamics during a 1 day simulation. ................................................13

Figure 16. Voltage regulator LTC positions over a 1 week period for baseline vs. 30\% PV....................14

Figure 17. Voltage fluctuations and load tap changes due to solar power ramping for baseline vs. 30\% PV. (a) Phase A, (b) Phase B, (c) Phase C. .........................................................................14

Figure 18. Cumulative LTC operation counts for different PV penetrations. .........................................15

Figure 19. Cumulative LTC operation counts for different PV penetrations. .............................................15

Figure 20. Solar PV inverter model in OpenDSS [157]. ...................................................................16

Figure 21. The Southern Company's feeder circuit under investigation (30\% penetration by

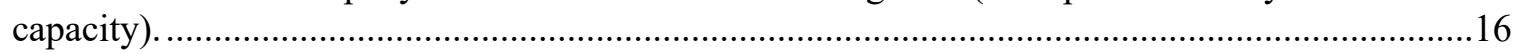

Figure 22. Feeder-distance voltage profile for the three cases under investigation................................17

Figure 23. LTC cumulative operations for 1 week (30\% percent PV by capacity). ................................17

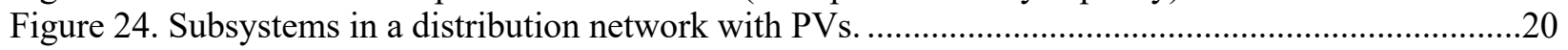

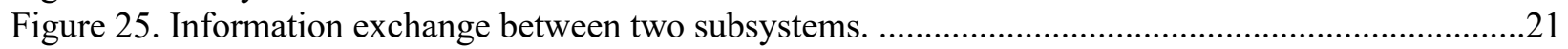

Figure 26. Flow chart of ADMM-based distributed optimal control of PVs..........................................21

Figure 27. Modified IEEE 33 node case with 15 PVs. .......................................................................22

Figure 28. Comparison of nodal voltages, network losses, and reactive power output of PVs.................22

Figure 29. Time-series distributed optimal PV control in distribution networks. ....................................23

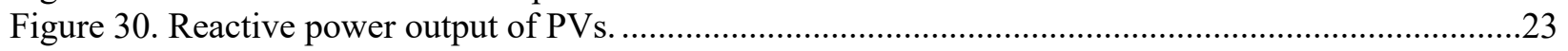

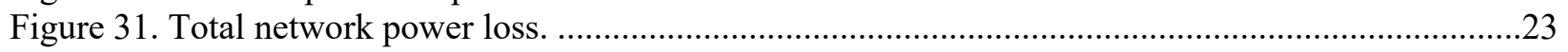

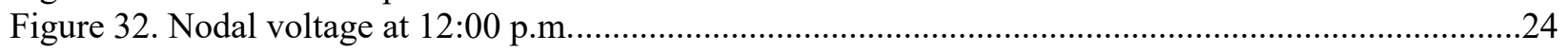

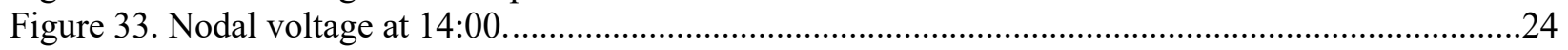

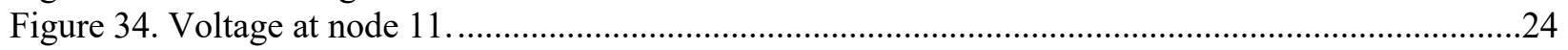

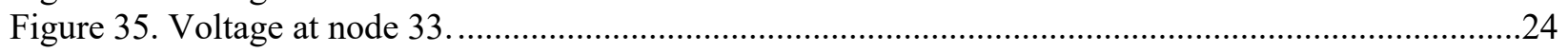

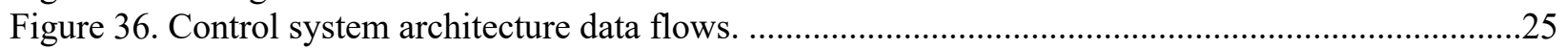

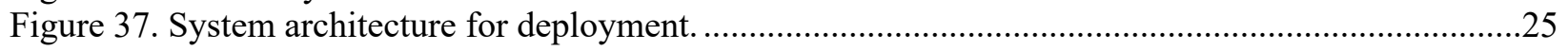

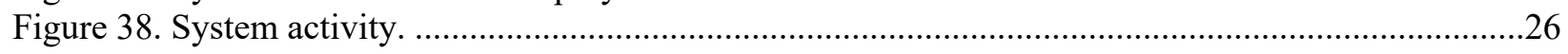

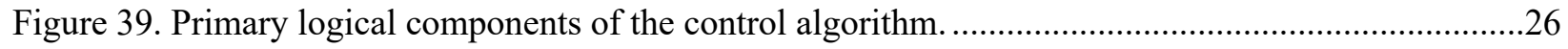

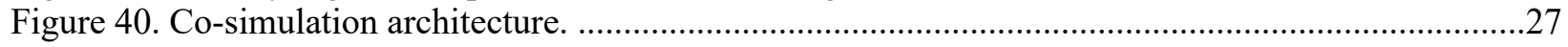


Figure 41. Simulation-based testing components for a fleet of TCLs.

Figure 42. Comparison of indoor temperatures for 45 buildings by using (a) MPC and (b) traditional control.

Figure 43. Comparison of control signals for (a) incremental control signal (fourth state) and

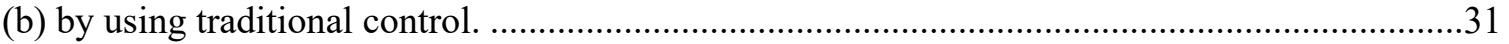

Figure 44. Comparison of tracking performance by using (a) MPC and (b) traditional control. ................32

Figure 45. Comparison of battery usage by using (a) MPC and (b) traditional control...........................32

Figure 46. Duty cycle and energy consumption comparison for Building 18 for (a) basic control and (b) MPC.

Figure 47. Duty cycle and energy consumption comparison for average building for (a) basic

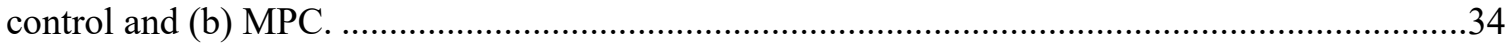

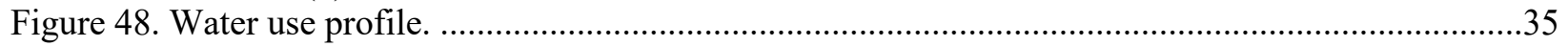

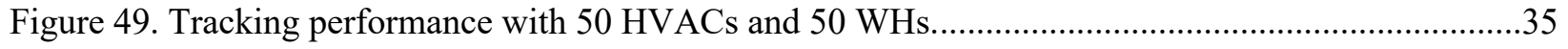

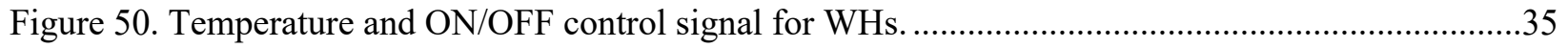

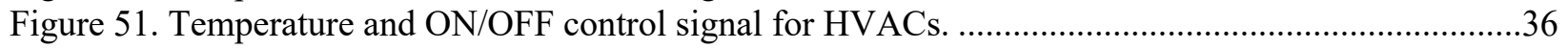

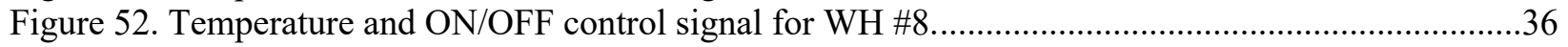

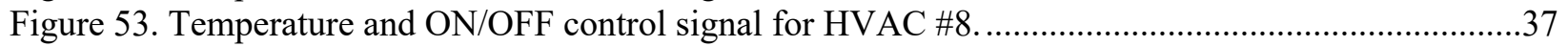

Figure 54. Simulation results for 100 ON/OFF HVAC systems using PuLP..........................................37

Figure 55. Daily HVAC switching count with (a) 50\% and (b) $30 \%$ PV penetration...............................38

Figure 56. Tracking performance for PV forecasting error by (a) 20, (b) 30, (c) 40, (d) 50, and (e) 60 min persistence intervals and (f) their corresponding tracking errors. ....................................38

Figure 57. Tracking performance for PV forecasting error represented by (a) 10, (b) 20, (c) 30, (d) 40 , and (e) $50 \%$ noise level and (f) their corresponding tracking errors. ........................................39

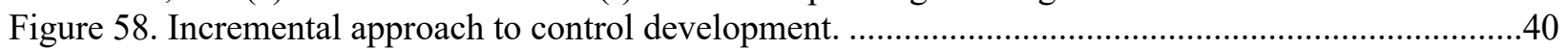

Figure 59. Controller infrastructure with external data sources.......................................................41

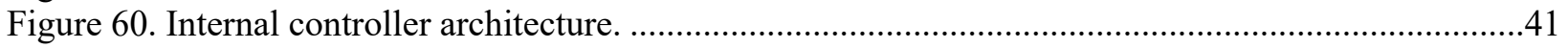

Figure 61. State-space model estimation via least-square system identification vs. true state-space

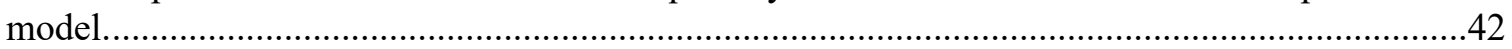

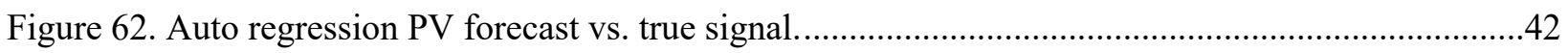

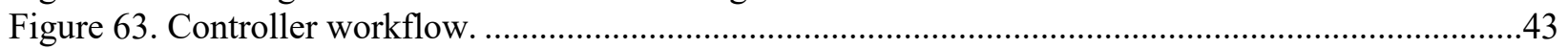

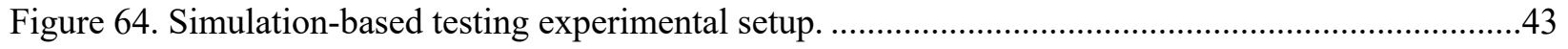

Figure 65. Simulation-based testing PV tracking and temperature data............................................44

Figure 66. State-space model estimation via least-square system identification with (a) $5 \%$ noise

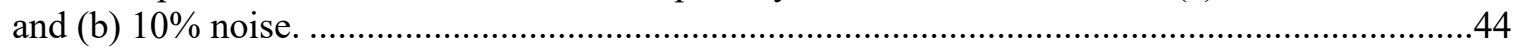

Figure 67. PV tracking and temperature measurements with (a) 5\% noise and (b) $10 \%$ noise..................45

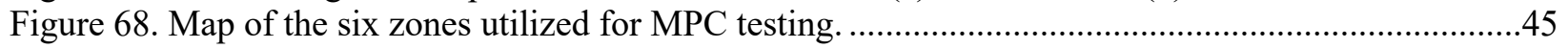

Figure 69. Ecobee thermostat in enclosure (left) with faceplate attached (right). ...................................46

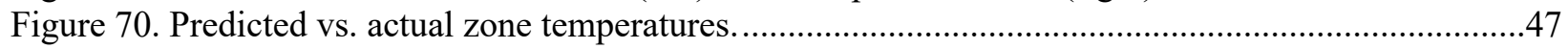

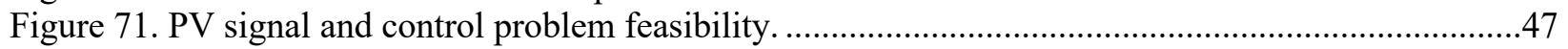

Figure 72. (a) MPC PV tracking test \#1 and (b) zone temperatures test \#1 . ..........................................47

Figure 73. (a) MPC PV tracking test \#2 and (b) zone temperatures test \#2. ...........................................48

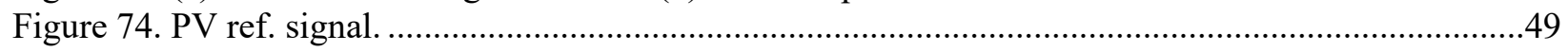

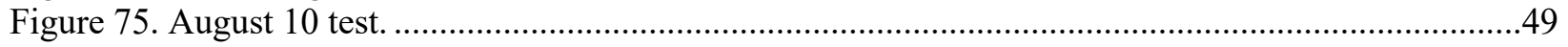

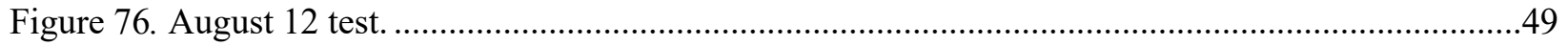

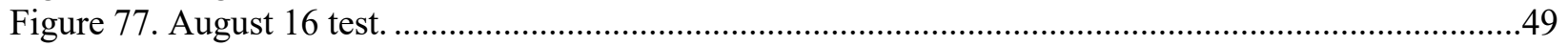

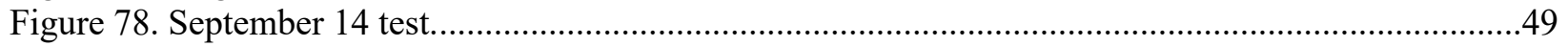

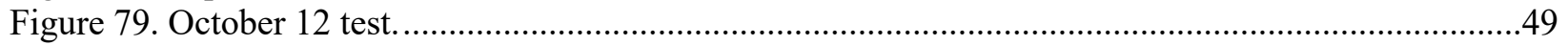

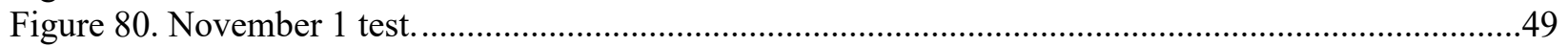

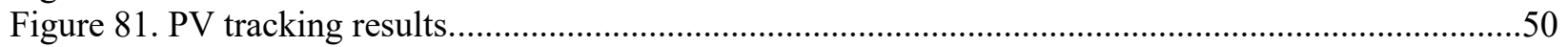

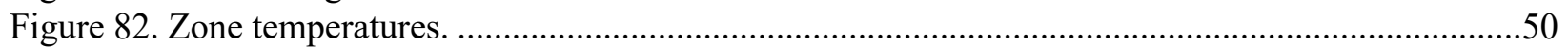

Figure A-1. Solar PV configuration from cell to array...................................................................... A-65 


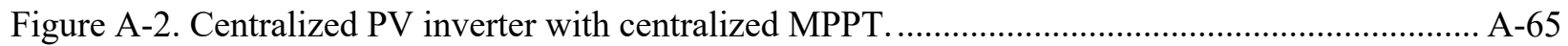

Figure A-3. PV inverter configurations in a large-scale solar farm................................................... A-66

Figure A-4. Centralized inverter with distributed MPPT at string level with low-voltage DC link....... A-67

Figure A-5. Centralized inverter with medium-voltage DC transmission configuration [70]............... A-67

Figure A-6. Centralized PV inverter with cascaded H-bridge configuration [75] .............................. A-68

Figure A-7. Centralized PV inverter with differential power processing (DPP) configurations:

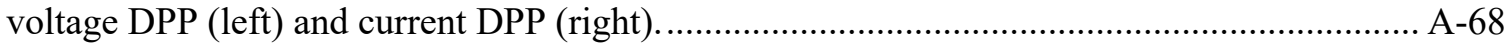

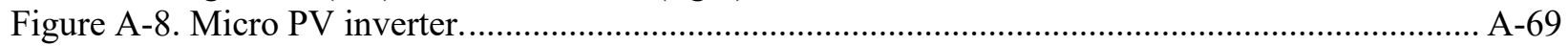

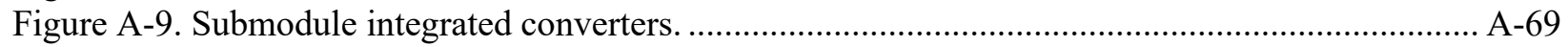

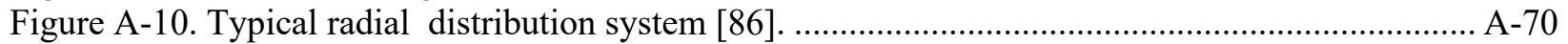

Figure A-11. Secondary grid network distribution system [87] .................................................. A-70

Figure A-12. Distribution system voltage regulation zones [156] ................................................ A-71

Figure A-13. Conventional voltage and reactive power control in distribution systems [93] .............. A-72

Figure A-14. Control architecture with and without remote monitoring [91] ................................... A-73

Figure A-15. The SCADA rule-based VVO approach [94] ............................................................. A-73

Figure A-16. The DMS model-driven IVVC scheme [94] ............................................................. A-74

Figure A-17. Topology of a realistic MV distribution feeder system in New South Wales,

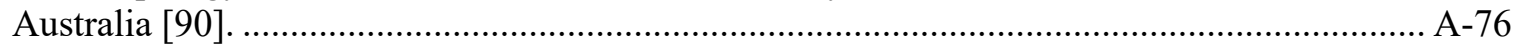

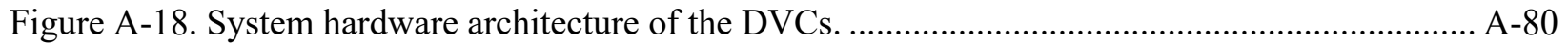

Figure A-19. One-line circuit of IEEE 13-Node Test Feeder............................................................. A-82

Figure A-20. Annual load profile for single-phase and three-phase loads. ........................................ A-83

Figure A-21. Annual solar PV generation profile...................................................................... A-83

Figure A-22. Solar PV generation in 1 week in August at different penetration levels. ....................... A-85

Figure A-23. Steady-state voltage profile with no solar PV ........................................................ A-86

Figure A-24. Steady-state voltage profile with 50\% solar PV penetration........................................ A-86

Figure A-25. Solar PV generation for a typical day in August..................................................... A-87

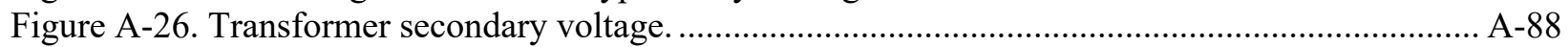

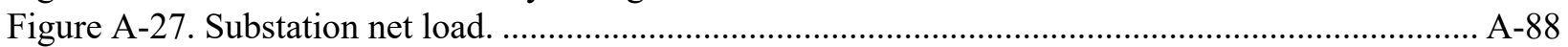

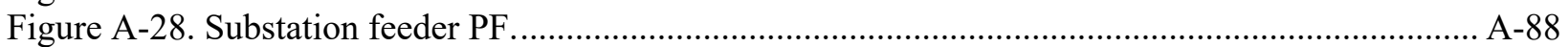

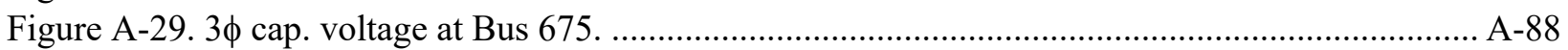

Figure A-30. Single-phase capacitor voltage at Bus 611 (Phase C)............................................... A-89

Figure A-31. Tap changes (left: no PV, right: with 50\% PV). ................................................... A-89

Figure A-32. Cumulative regulator tap changes over a 1 year period for different solar PV

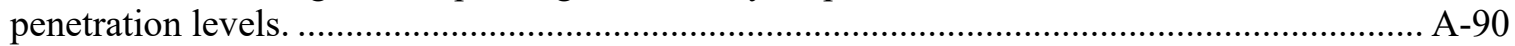

Figure A-33. Proposed DVC control structure ......................................................................... A-92

Figure A-34. A simple radial distribution feeder circuit with DVC communication scheme. ............. A-93

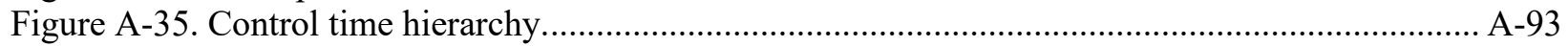

Figure A-36. Voltage control curve smoothed by a sigmoid function [48] ........................................ A-95

Figure A-37. Voltage control coordination at different timescales. .................................................... A-96

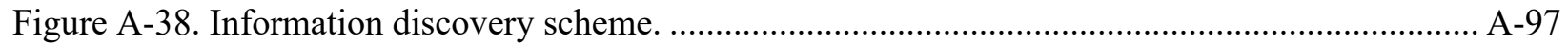

Figure A-39. Context of distributed optimization and control problem. ......................................... A-97

Figure A-40. Framework of proposed multi-timescale co-simulation platform............................... A-101

Figure A-41. An experimental implementation of the co-simulation platform. ............................... A-101

Figure A-42. Power network partition for co-simulation. .......................................................... A-102

Figure A-43. Convergence of the objective functions. $\alpha=1.0$ is the general ADMM, and $\alpha=1.8$

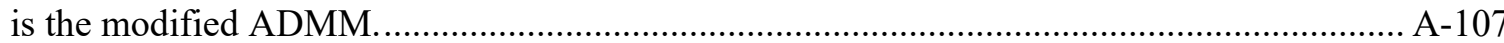

Figure A-44. The coordination between distributed optimal PV control and building control in

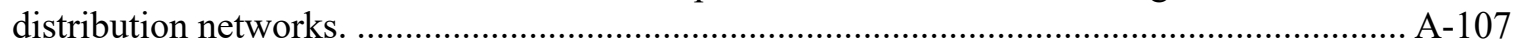

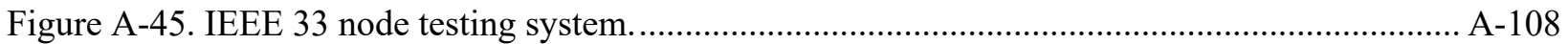

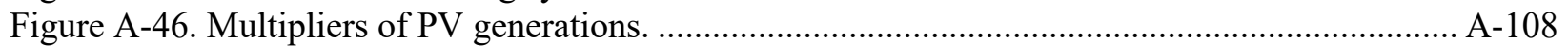

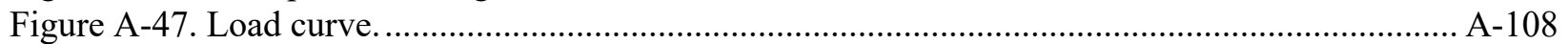


Figure A-48. Comparison between the proposed model and OpenDSS.

Figure A-49. Comparison between the proposed method and constant PF control.

Figure A-50. Comparison of distributed optimal PV control with and without coordination with building control.

Figure A-51. Inputs and output of the HVAC model system.

Figure A-52. Evaluating multiple estimated models, $\lambda$ is the regularization parameter of N2SID..... A-113

Figure A-53. Accuracy analysis of the estimated model.

Figure A-54. Validating the entire dataset using a single model.....

Figure A-55. Accuracy analysis of the model estimated with RN2SID.

Figure A-56. Order of the model estimated with RN2SID.

Figure A-57. (a) Self-validation and (b) inputs given to the plant and the estimated model............... A-115

Figure A-58. (a) Cross-validation and (b) inputs given to the plant and the estimated model............. A-115

Figure A-59. RMSE of indoor temperature prediction for different prediction horizons using LS and compared with SID.

Figure A-60. Prediction of indoor temperatures using LS for several prediction horizons: $15 \mathrm{~s}, 1$ min, 5 mins, 10 mins, 30 mins, and 60 mins.

Figure A-61. Performance of PV power output prediction using AR(2)....

Figure A-62. Performance of PV power output prediction using ARIMA(2,1,2).

Figure A-63. Indoor temperatures obtained by using (a) MFC, (b) MPC, and (c) total energy consumption by 100 buildings obtained with MFC and MPC vs. generated PV energy. ....... A-121

Figure A-64. The duty cycles for 100 HVAC systems using (a) MFC and (b) MPC......................... A-122

Figure A-65. The total number of state switching for 100 HVAC systems using (a) MFC and (b) MPC.

Figure A-66. Error in indoor air temperature for cases of unconstrained and constrained control input $\left({ }^{\circ} \mathrm{C}\right)$.

Figure A-67. (a) Control input for cases of unconstrained and constrained control input. (b) Indoor air temperature for cases of unconstrained and constrained control input.

Figure A-68. Indoor temperatures and control inputs for 100 residential and 100 commercial buildings controlled by using MFC without PV power constraint.

Figure A-69. Total power consumed by 100 residential and 100 commercial buildings controlled by using MFC without PV power constraint and compared with the generated PV power.... A-127

Figure A-70. Projection of the ideal number of residential and commercial HVAC units to turn on $(\boldsymbol{c 1}, \boldsymbol{c 2})$ onto the constraint line in Eq. (A-62). A-128

Figure A-71. Indoor temperatures, control inputs, and total power consumed for 100 residential and 100 commercial buildings controlled by using MFC with PV power constraint.....

Figure A-72. Indoor temperatures, control inputs, and total power consumed for 100 residential and 100 commercial buildings controlled by using MFC with PV power constraint............. A-130

Figure A-73. Indoor temperatures, control inputs, and total power consumed for 100 residential and 100 commercial buildings controlled by using MFC with PV power constraint............. A-131

Figure A-74. Mapping importance weight to projection angle. A-132

Figure A-75. Indoor temperatures and total power consumed for 100 residential and 100 commercial buildings controlled by using MFC with PV power constraint $(w=0.9)$

Figure A-76. Indoor temperatures and total power consumed for 100 residential and 100 commercial buildings controlled by using MFC with PV power constraint $(w=0.5)$

Figure A-77. Indoor temperatures and total power consumed for 100 residential and 100 commercial buildings controlled by using MFC with PV power constraint $(w=0.15) \ldots \ldots \ldots . . . . \mathrm{A}-135$

Figure A-78. Indoor temperatures and total power consumed for 400 residential HVAC units controlled by using MFC with equal priorities and PV power constraint.

Figure A-79. Indoor temperatures and total power consumed for 400 residential HVAC units controlled by using MFC with different priorities and PV power constraint. 
Figure A-80. Indoor temperatures and total power consumed for 100 residential HVAC units, 100 commercial HVAC units, and 100 WHs controlled by using MFC with equal priorities and PV power constraint.

Figure A-81. Indoor temperatures and total power consumed for 100 residential HVAC units, 100 commercial HVAC units, and 100 WHs controlled by using MFC with different priorities and PV power constraint.

Figure A-82. Indoor temperatures and total power consumed for 100 residential HVAC units, 100 commercial HVAC units, and 100 WHs controlled by using MFC with different priorities and PV power constraint.

Figure A-83. Control architecture.

Figure A-84. Device/data management and optimization linked by the database.

Figure A-85. Control flow.

Figure A-86. Application flow diagram.

A-141

Figure A-87. Timing diagram for the MPC controller. A-142

Figure A-88. Control workflow for the MPC control. A-142

Figure A-89. The architecture of the UA tool (GURA-W) A-143

Figure A-90. Power duration curve for a building power consumption. A-143

Figure A-91. Sensitivity analysis results for a building power consumption. A-143

Figure A-92. Frequency-domain analysis of frequencies for a building power consumption.............. A-144

Figure A-93. Sensitivity analysis results for the frequencies in the building power consumption....... A-144

Figure A-94. Value of the postulated measure with the value of the measure based on EnergyPlus results.

Figure A-95. Distribution of the prediction error after a certain time.

Figure A-96. RC building model calibration using the MFU quantification process. A-150

Figure A-97. The MFU quantification in the RC model over 144 time steps. A-150

Figure A-98. The temperature prediction with SU compared with the actual temperature. A-151

Figure A-99. Control information flow. A-152

Figure A-100. In vitro testing in simulation test bank. A-153 


\section{LIST OF TABLES}

Table 1. WEBB peak load based on 15 min measurements from July 1 to September 1, 2016................10

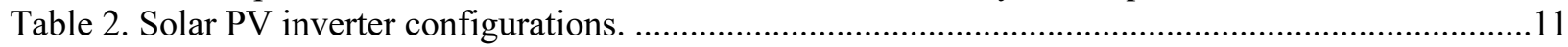

Table 3. HVAC model parameter definitions. ........................................................................................29

Table A-1. Number of conflicting operations associated with DG voltage-regulating device interactions, as found in Ranamuka et al. [90]................................................................. A-76

Table A-2. Solar power generation at different penetration levels. ................................................... A-85

Table A-3. Comparison results of the two methods........................................................................ A-107

Table A-4. Evaluation of the accuracy of the estimated model. ..................................................... A-116

Table A-5. The standard statistical methodology to construct AR and ARIMA models. ................... A-118

Table A-6. Comparison of MFC and MPC using tracking error metrics. ......................................... A-121

Table A-7. Comparison of MFC and MPC in terms of HVAC duty cycle and number of state

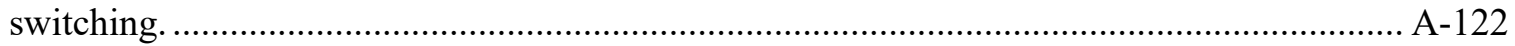

Table A-8. Experimental setup for the controllability test.......................................................... A-146

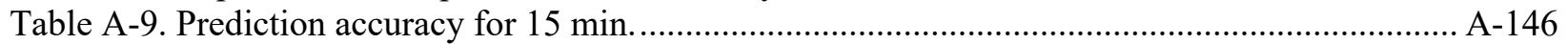

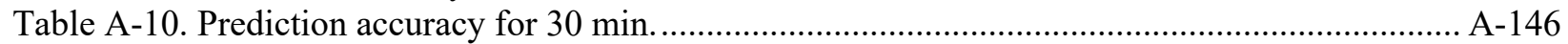

Table A-11. The average prediction accuracy of the RC model over all uncertainty runs.................. A-148

Table A-12. Temperature prediction accuracy. ................................................................................. A-149

Table A-13. Temperature prediction accuracy. ................................................................................. A-151 


\section{ACKNOWLEDGMENT}

This material is based upon work supported by the U.S. Department of Energy, Office of Energy Efficiency and Renewable Energy, the SunShot National Laboratory Multiyear Partnership (SuNLaMP) program under Award Number 31081. 



\section{EXECUTIVE SUMMARY}

Solar photovoltaic (PV) resources are the most common form of distributed generation in residential and commercial customer premises within electric distribution networks. A higher penetration of PV generation in distribution circuits will impose challenges on maintaining service voltages within the range of industry standards, power quality, and power flow. Buildings consume $74 \%$ of the electricity produced in the United States, and a significant portion of the building load is dispatchable, making them responsive to electrical grid needs. Oak Ridge National Laboratory-in collaboration with Southern Company; the University of Tennessee, Knoxville; and the Georgia Institute of Technology - is examining the PV integration issues in distribution-level electrical grids and developing integrated demand-side control and communication systems to enable responsive loads. The proposed responsive loads mechanism performs renewable generation following to increase the penetration of solar PV within each feeder. The specific objectives of this project are to (1) examine distribution-level PV integration scenarios to understand requirements, (2) undertake an end-to-end simulation-based design of a distributed control strategy of loads geographically near the PV generation asset to minimize the effect on the distribution feeder, (3) deploy and demonstrate the control technology developed in partnership with utilities, and (4) perform a scalability analysis at the utility scale.

This 3-year integrated project aims to develop, demonstrate, and validate demand-side control technology to enable increased the penetration of renewables while mitigating challenges that arise due to their intermittency. Activities in Budget Period (BP) 1 focused on a literature review and the formal design of a control system for integrating local distribution with generation and loads. The team used modeling and simulation to evaluate the impact of varying buildings loads, variable PV generation, and power flow dynamics on the distribution circuit. The dynamic models developed in BP 1 were used in BP 2 to develop a model-based control design and a test bed. The test bed has enabled the simulation-based testing and comparison of different control designs and formulations applied to different configurations of the distribution grid, PVs, and building loads. The control approaches developed in BP 2 were implemented in BP 3 in the form of hardware deployed at the Central Baptist Church (CBC) in Knoxville, Tennessee, for testing and evaluation.

The outcome of this project was the development and demonstration of open-source, low-cost, low-touch sensing and control retrofits to distributed PV generation and building loads that, in a coordinated fashion, provide the load-shaping response needed to integrate high levels of renewable penetration. This research addresses the target metrics by dynamically controlling a load with solar generation variability to minimize the extent of two-way power flow, enhance reliability, facilitate high PV penetration ( $>100 \%$ of peak load in a line segment), and generate scalable software and hardware solutions adaptable to any penetration levels. The research and development activities are focused and designed to be impactful within the relevant 2020 targets time frame.

An accurate open-source integration simulation framework for end-to-end control design was developed and deployed at the CBC facility for testing and evaluation. This final report provides a detailed review of the technical results achieved during this 3-year integrated project. A novel spectral analysis of PV data is demonstrated to derive the requirements of the control design. A detailed simulation-based analysis of PV integration at increasing penetration levels is presented using 1 year of PV data to demonstrate the impact on the distribution circuits. Two different control strategies were developed and demonstrated via simulation to track variable PV generation with adaptive load dispatch. The report concludes with a summary of accomplishments and recommendations for a path forward. 



\section{BACKGROUND}

Solar photovoltaic (PV) resources are the most common form of distributed generation (DG) in residential and commercial customer premises within an electric distribution network. Rooftop solar PV units can serve part of the local loads, thereby decreasing stress on the distribution feeder and improving overall system performance (e.g., by reducing feeder losses). However, the large, rapid swings in power output from a very large numbers of PV resources can have the opposite effect, increasing stress on the distribution feeder, degrading system performance, and consequently creating serious challenges for distribution network operators. When PV generation is greater than the local demand, the excess power flow from PV inverters produces a reverse power flow in the feeder. This creates a voltage rise, possibly causing a violation of node voltage limits, and reduces the useful life of transformers due to rapid tap adjustments that are made while attempting to regulate the feeder voltage [1]. These observed effects are a clear sign that conventional forms of voltage regulation, which are predicated on power flowing in one direction, are insufficient to incorporate large amounts of solar power. The present inability to regulate voltage in the presence of high PV penetration is a primary cause of the relatively limited PV deployments seen in current circuits that support largely residential and commercial loads.

Existing control strategies can be enhanced to overcome this problem by introducing responsive loads that perform renewable generation following. By causing demand for electricity to follow the availability of solar power, we can significantly reduce or even eliminate the reverse power flows across the feeder and the associated undesirable voltage fluctuations. There are two general methods for managing loads: indirect load control and direct load control. With indirect load control, the power consumption of a load is controlled manually by the energy customer or automatically by software within the load per local considerations (e.g., electricity prices). With direct load control [2-4], the power consumption of a load is controlled by the utility or system operator, regardless of the customer's local circumstances. With direct local control, it is possible to precisely adjust electricity use, but it is difficult to gain customer acceptance if local needs are compromised. International experiences with direct and indirect load control are reviewed in Heffner et al. [5] in which the authors identify specific approaches that have enabled customer loads to effectively participate in power system control.

Several types of distributed energy resources (DERs) have been proposed to augment direct and indirect control systems, including battery storage [6], plug-in electric vehicles [7-9], electrically powered residential water heaters (WHs) [10-11], and thermostatically controlled loads (TCLs) used for residential space heating and cooling [12-15]. WHs and TCLs are interesting because they store energy but do so in a thermal form rather than an electrical form. These loads can generally withstand relatively frequent, short interruptions without a significant reduction in end-use performance. However, these DERs are relatively small and require a communication infrastructure to aggregate sufficient numbers of devices to perform as a useful control resource.

A significant amount of research has focused on aggregating many small, individually controlled on/off TCLs [16-20]. The results indicate that low-dimensional controls, such as global adjustments of thermostat settings or switching the state of many individual TCLs, can create hour-long correlations between the dynamics of individual TCLs. Several approaches have been pursued, including statistically based methods [16], linear-response transfer functions [14-15], state estimation-based methods [17], and deterministic protocols [13]. Based on this body of work, aggregating many small, residential-scale TCLs for a relatively fast and flexible demand response (DR) appears to be feasible.

TCLs - such as air-conditioning systems, heating systems, and WHs - are present in most residential and commercial buildings, and they are particularly well suited to large-scale, distributed control [16]. However, these types of loads present difficult control challenges that arise from the complex, nontrivial thermodynamic and hydrodynamic coupling between the individual cooling and/or heating loads and the 
many state variables that must be handled. Although the dynamics of large commercial heating, ventilation, and air-conditioning (HVAC) systems in particular might be complex, a low-dimensional control is economically necessary (i.e., the control must use relatively few inputs and outputs to enable accurate control over the total HVAC power consumption while keeping a building's occupants comfortable).

To provide a response that can influence the performance of the distribution circuit, many thermostats must be "ganged" together so that their operations can be coordinated. This method has been used in several other works [17-21] for infrequent DR, such as peak shaving, and in Goddard, Klose, and Backhaus [18] for faster DR control. Lu [22] presents the direct control of HVAC units that follows regulation signals and proposes a direct load control algorithm that provides intra-hour load-balancing services by using aggregated HVAC loads. This could enable small residential or commercial customers to participate in ancillary service markets in the future. Callaway [12] proposes a system identification approach based on Fokker-Planck diffusion models to design a direct control strategy to manage large numbers of HVAC units. An extended optimal centralized control strategy with comfort-constraints is proposed in Parkinson et al. [23] and Wang et al. [24]. This method was implemented on a simulation test bed to investigate the regulation and load-shifting service supported by HVAC units to offset the intermittency of renewable resources in a self-regulating distribution system. Katipamula and $\mathrm{Lu}$ [25] studied various residential HVAC load control strategies that can be employed to mitigate price volatility. Yoon, Bladick, and Novoselac [26] propose a controller that can be used to control an HVAC system in response to the retail price of electricity.

To summarize this review, buildings can play a significant role in fast DER programs. For example, it is estimated that some buildings can shed $60 \%$ of HVAC demand for $2 \mathrm{~h}$ load shed events and $80 \%$ for 20 min events for facilities with rooftop chiller units that can turn off compressors [27]. Large office and college buildings that can make set point adjustments to reduce demand can shed $50 \%$ for both $2 \mathrm{~h}$ events and 20 min events [27]. Based on prior research, refrigerated warehouses - particularly mediumtemperature refrigeration systems - are known from to be able to reduce their loads by at least $25 \%$ for $2 \mathrm{~h}$ without a serious change in temperature. Data centers can temporarily reduce their HVAC use also. Such loads are a resource that can give grid operators the tool they need to manage an electrical grid that receives an intermittent supply from a high percentage of renewable power sources.

The high penetration of utility-scale solar PV power will inevitably bring challenges to power system planning and operations [28-29] due to the variability and nonexistent or limited capability to dispatch solar power. Because the PV inverters' objective is to maximize energy output, changes in solar irradiance directly impact the produced power. The burden and expense for system operators to include solar generation come in the form of an increased need for fast-ramping spinning reserves. One typical method for addressing this challenge is to integrate advanced solar forecasting techniques into the operations center.

The impact on system protection is more eminent in the radial distribution system. Traditional distribution systems are designed for unidirectional power flow from a substation bus to the feeder end. Accordingly, the protection devices react to the current flow from the bus side in case of short-circuit fault. With solar PV installations on the feeder, bidirectional power flow will occur and could unnecessarily trip circuit breakers and reclosers. Moreover, the short-circuit fault current contribution is dramatically smaller from a PV system than from a synchronous generator, which brings challenges when setting relays and might require increased low current-sensing accuracy.

The impact of solar PV systems on distribution feeder voltage has been investigated in several US Department of Energy projects, such as the Renewable System Interconnection Study, and the High Penetration PV Integration, as well as research papers [30-34]. One common finding is that an 
overvoltage event could be triggered when the solar PV system is installed at the end of a feeder. During high PV generation and low load periods, voltage rise could occur due to reverse power flow. Active power curtailment of PV inverters [31] and the integration of distributed energy storage systems [32] can help address overvoltage issues. A coordination strategy of various reactive power resources-including substation electronic load tap changer (LTC), capacitor bank, energy storage, and PV inverter reactive power injection - is required to optimally manage the distribution voltage profile while minimizing the solar power curtailment.

Power converters serve as the interface between PVs and the power grid. These converters are crucial for power conversion and conditioning, maximum power point (MPP) tracking, and potential grid ancillary services. In this project, the team developed an end-to-end proactive automated control of responsive building loads with the objective to minimize voltage impacts due to high penetrations of PVs while maintaining customer comfort.

\section{PROJECT RESULTS AND DISCUSSION}

\subsection{SPECTRAL ANALYSIS OF SOLAR PV POWER OUTPUT}

This section presents the spectral analytics of solar PV power generation and the power consumption of multiple building TCLs. Spectral analytics of solar PV power provide an understanding of the PV frequency content due to solar irradiance and disturbance in PV generation. The analyses define the bandwidth over which TCLs can operate to verify that their frequency bandwidths match the one for the solar PV generation. This information can be used to optimally assign the TCLs in different buildings to the appropriate frequency band. Figure 1 illustrates the proposed approach. First, the spectral (i.e., frequency) contents of the collected time-domain solar PV and building TCLs power data are captured via Fourier transform operations. The filtered PV power is then fed to the controller along with other parameters to dispatch different building TCLs that match the corresponding PV frequency content (i.e., timescale).

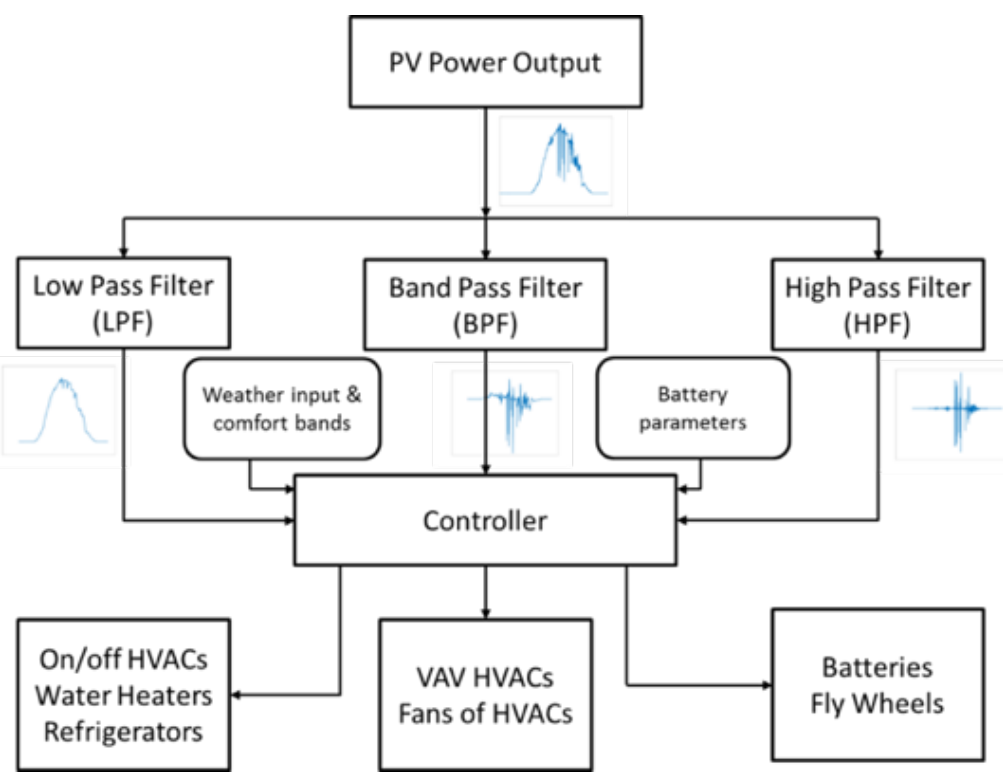

Figure 1. The proposed PV power filtering approach. 
The frequency-domain analysis was conducted on 12-month PV and buildings' TCLs measurement power data. The PV measurement power data were collected from a 13-kW solar PV panel located on the roof of a building at Oak Ridge National Laboratory (ORNL). The relatively fast, medium, and slow frequency bands illustrated in Figure 2(a) are based on measured solar power output recorded during a sample day, where $L P F$ stands for low-pass filter, BPF stands for band-pass filter, and HPF stands for high-pass filter. Figure 2(b) shows the time-domain PV power profile for one summer day and its corresponding low- $(<1 \mathrm{mHz})$, medium- (1-10 mHz), and high- $(>10 \mathrm{mHz})$ frequency contents.

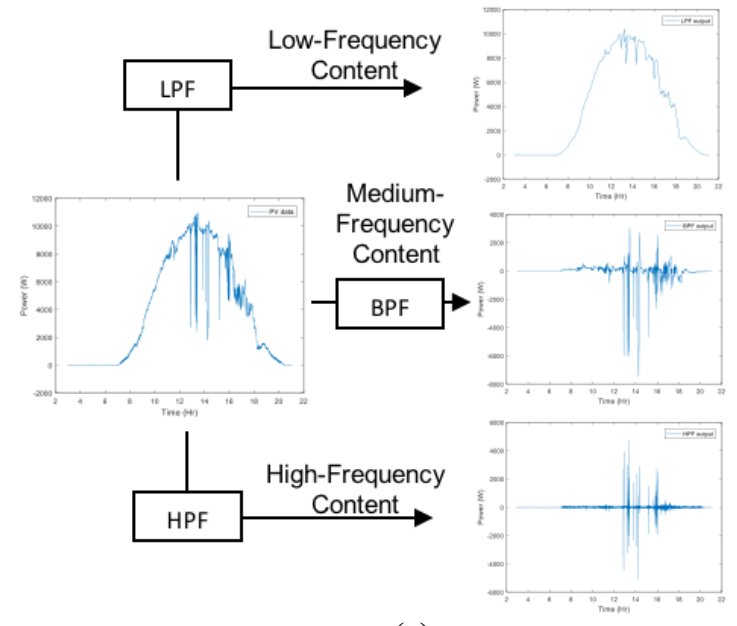

(a)

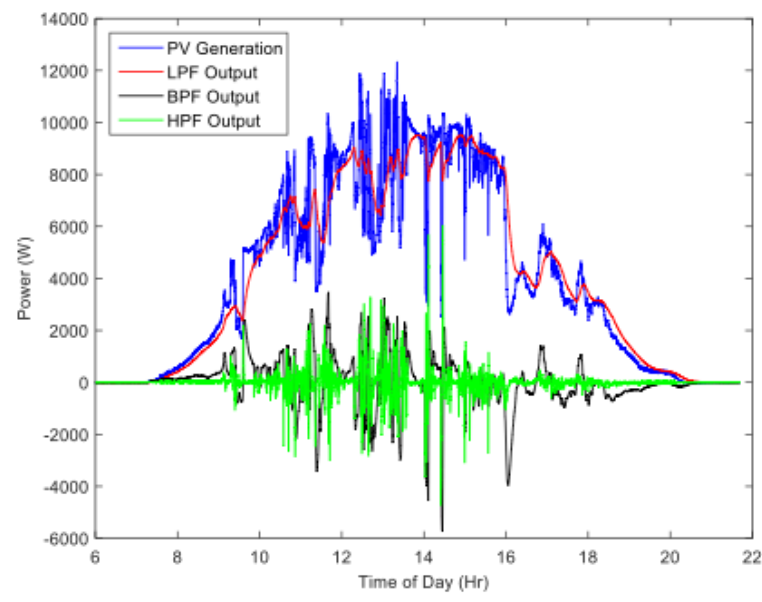

(b)

Figure 2. (a) Filtering process of input PV power; (b) PV power profile for one summer day in 2015 and its corresponding low-, medium-, and high-frequency content.

The time-domain PV power data are converted to frequency-domain data via the Fourier transform operation [35]. Figure 3 shows $24 \mathrm{~h}$ time-domain PV power profiles for eight summer days selected at random and their corresponding frequency-domain profiles. Significant generation variability (i.e., fluctuations) in the time-domain power profiles is illustrated in Figure 3(a). In Figure 3(b), the spectral analysis of solar PV power data is observed to contain a wide range of frequencies (i.e., timescales), ranging from a few hertz and fractions of hertz (sub-seconds to seconds) to multiple millihertz (minutes) and fractions of millihertz (hours). This wide range in frequency can be divided into relative categories of fast, medium, and slow frequency ranges. 


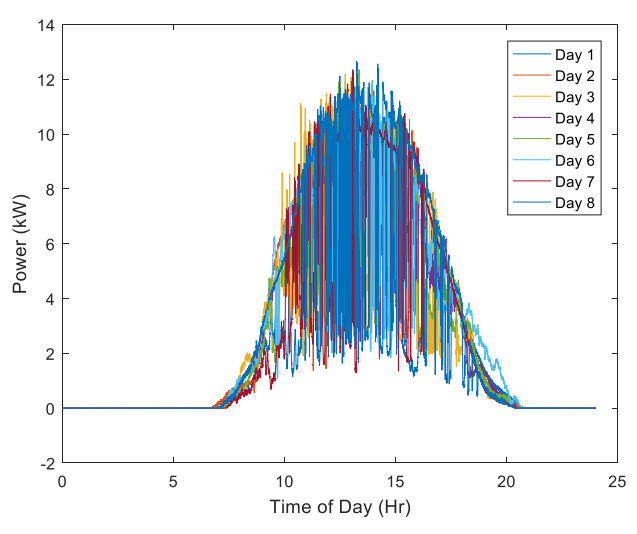

(a)

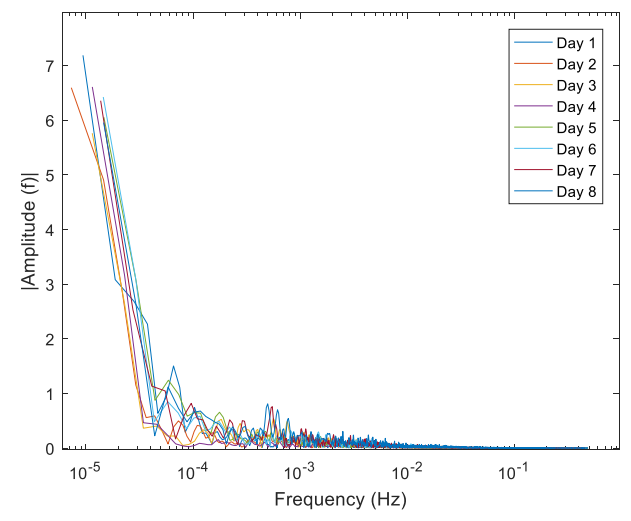

(b)

Figure 3. (a) The time-domain solar PV power profiles for eight summer days collected from a 13-kW solar $P V$ panel showing fast and large fluctuations of PV outputs and (b) their corresponding frequency-domain power profiles.

Most of the PV energy is found at frequencies lower than $1 \mathrm{mHz}(\sim 15 \mathrm{~min})$. Also, only a small amount of $\mathrm{PV}$ energy is found at frequencies between 1 and $100 \mathrm{mHz}(\sim 15 \mathrm{~min}$ to $10 \mathrm{~s})$. PV energy at frequencies higher than $100 \mathrm{mHz}$ is almost negligible. The low-frequency content corresponds to daily solar power variations, which are mainly from the parabola shape in the time-domain power profiles. Mediumfrequency contents come from changes in solar irradiance due to temporary cloud cover and other factors. High-frequency contents come from changes due to other disturbances (e.g., passing flocks of birds, local intermittent shading).

The spectrogram [36] is used to visualize the time-frequency content of the PV power. It visualizes how the PV power spectrum of frequencies varies with time and is used to compute the time-frequency power spectral density (PSD) [37-38]. Figure 4 demonstrates the time-frequency PSD for the PV power output for one randomly selected summer day. The high-frequency content, which corresponds to the fast fluctuations of the PV power in the middle of the day, has been captured at the true time moments.

Boxplots [39] are used to visualize such statistics of PV frequency content. The lower and upper lines of the "box" represent the 25th and 75th percentiles of the sample,

respectively; the line in the middle of the box is the sample median; and the extended lines from each end of the box represent the minimum and maximum values in the sample. Figure 5(a) illustrates the 2015 annual statistics of the PV power output frequency contents. About 98\% of the PV energy is located at the lowfrequency band (lower than $1 \mathrm{mHz}$ [ 15 min]). Also, only a little of the PV energy (about 2\%) is found at frequencies between 1 and $100 \mathrm{mHz}$ ( $\sim 15 \mathrm{~min}$ to $10 \mathrm{~s}$ ). PV energy at frequencies higher than $100 \mathrm{mHz}$ is almost negligible and is not shown in Figure 5. The dispersion from the medians is very large, especially at low

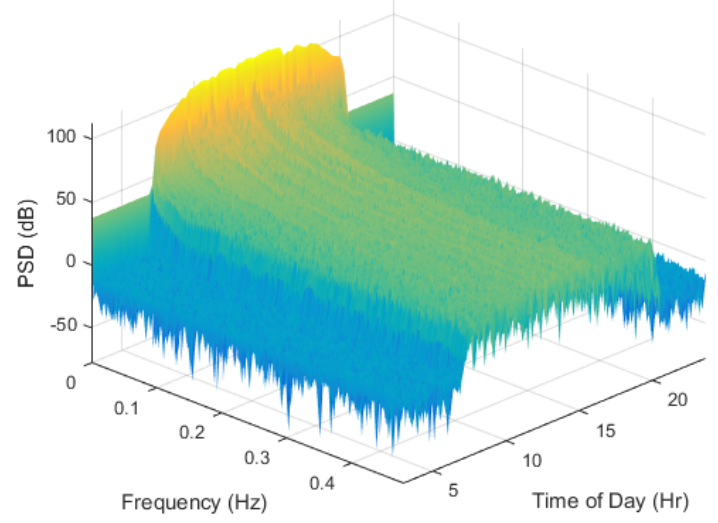

Figure 4. The PV power output time-frequency PSD for one summer day, illustrating how the PV power spectrum of frequencies varies with time. 
frequencies (e.g., $\sim 3 \mathrm{~kW}$ at the $0.01 \mathrm{mHz}, \sim 0.3 \mathrm{~kW}$ at the $0.05 \mathrm{mHz}$ ) due to many factors, such as daily/hourly variations and diffuse/beam solar irradiance. Figure 5(b) illustrates the 2015 seasonal statistics of the PV power output frequency contents. The median powers are highest in summer, moderate in fall and spring, and lowest in winter at this location.

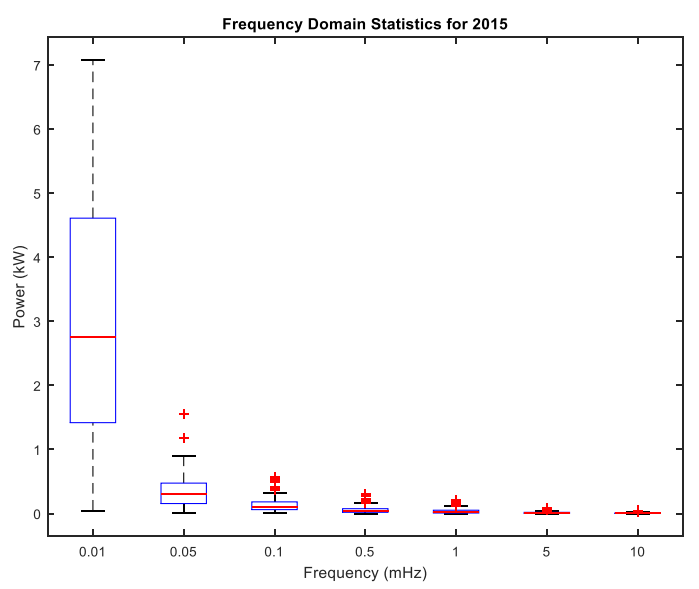

(a)
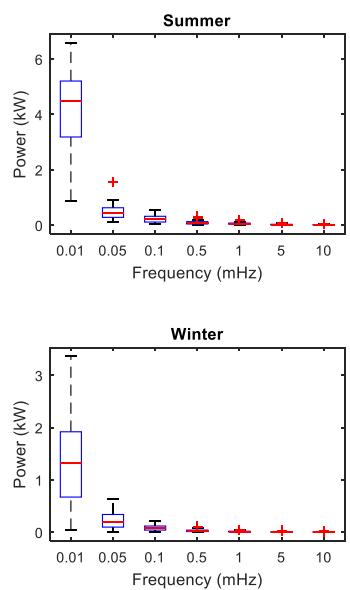

(b)
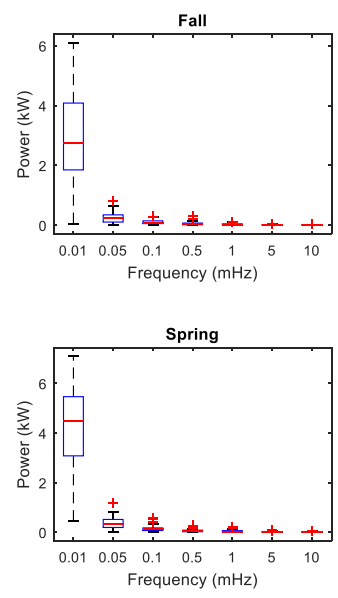

Figure 5. Boxplots illustrating the 2015 (a) annual and (b) seasonal statistics for the spectrum of PV power outputs.

The residential homes and commercial buildings are usually equipped with batteries for energy storage. The batteries are sized based on the solar PV rating [40]. For example, for the solar PV power output shown in Figure 2, total energy over 1 day that must be stored is $61.5 \mathrm{kWh}$, assuming that all solar PV energy is going to the battery. However, a spectral analysis of solar PV helps divide PV power to low-, medium-, and high-frequency components. This report shows that HVAC loads can be used to consume low and medium frequencies of solar PV power, which leaves high-frequency components. Batteries can be used to store/offset the high-frequency components. By doing so, the size of the battery is reduced because the amount of energy over 1 day that must be stored will be reduced significantly (only the highfrequency part of the PV generation is stored). In this case, for the solar PV profile in Figure 2, only $0.76 \mathrm{kWh}$ must be stored over that day. For this particular day, there is a huge reduction in total solar PV energy consumed by the battery over 1 day. Figure 6(a) shows battery states for the solar PV profile in Figure 2. Using these values, the state-of-charge (SOC) of the battery is illustrated in Figure 6(b). It is assumed that the initial SOC of the battery was $50 \%$. 


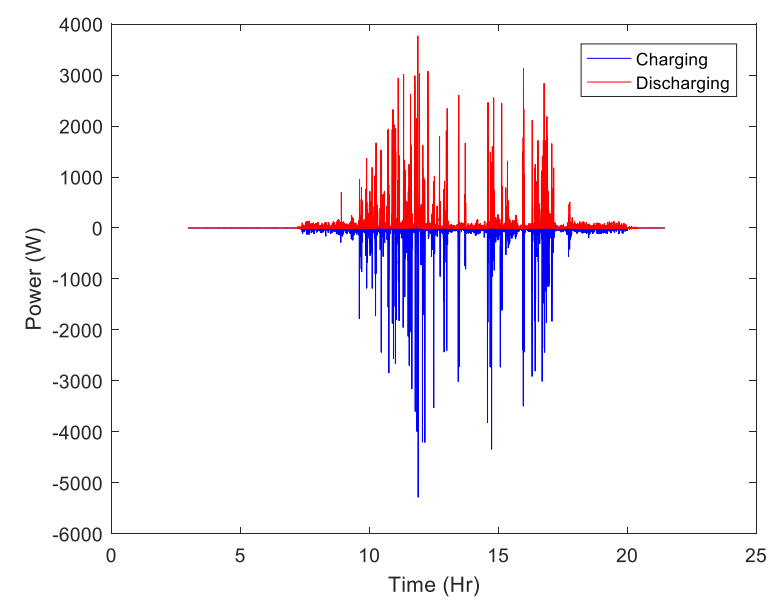

(a)

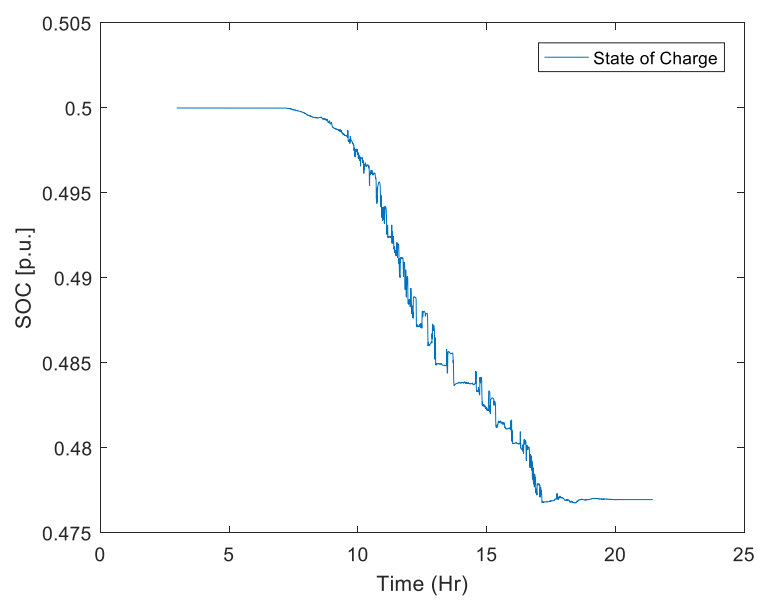

(b)

Figure 6. (a) Charging/discharging power levels and (b) SOC of the battery.

As seen in Figure 6, battery level changes from charging to discharging mode in seconds. Although there is almost negligible decrease in the SOC (about 2.5\%), the battery switches from discharging to charging mode 7,440 times in that day. This could damage the battery or reduce its life cycle. Another option is to use a flywheel storage system [41] to alleviate this problem.

\subsection{FREQUENCY-DOMAIN ANALYSES FOR THE VARIOUS BUILDING TCLS}

The team conducted frequency-domain analyses for the various building TCLs - such as WH, HVAC, and refrigeration - for optimal employment of these loads to accommodate the different frequency contents of PV generation. Figure 7(a) illustrates the annual statistics of the on/off HVAC power consumption frequency contents for a daily power consumption of a 3 ton HVAC unit recorded every 15 $\mathrm{s}$ for the year 2016. Most of the power consumed, which lies at frequencies less than $10^{-2} \mathrm{~Hz}$, corresponds to the low-frequency (less than $10^{-4} \mathrm{~Hz}$ ) and medium-frequency (between $10^{-4} \mathrm{~Hz}$ and $10^{-2} \mathrm{~Hz}$ ) bands of the PV-generated power. Figure 7(b) illustrates the annual statistics of the WH power consumption frequency contents for a daily power consumption of a 50 gal tank unit with $4 \mathrm{~kW}$ resistive heating elements and a 0.9 efficiency factor recorded at $1 \mathrm{~min}$ intervals during 2012. Figure 7(c) illustrates the 1 month statistics of the refrigerator power consumption frequency contents for the daily power consumption of a $4.5 \mathrm{~kW}$ refrigerator [42] recorded every $30 \mathrm{~s}$ for 2 months during 2016. These refrigeration compressor power consumption data are described in Fricke et al. [43]. Figure 7(d) illustrates the frequency spectrum of the average powers generated and consumed by PV/building TCLs for comparison. The frequency content of the average solar PV power output matches those for the on/off HVAC, WH, and refrigeration power consumptions. Results show that nearly all the PV output (about $98 \%$ ) is contained within frequencies lower than $1 \mathrm{mHz}$ (equivalent to $\sim 15 \mathrm{~min}$ ), which is compatible for consumption with local building TCLs.

A load duration curve is used in electric power generation to illustrate the relationship between generating capacity requirements and capacity use. A load duration curve is similar to a load curve, but the demand data are ordered in descending order of magnitude rather than chronologically. This concept was used to understand the demand of HVAC, WH, and solar PV generation over 1 year. This will determine power consumed over the year so that this information can be related with solar PV generation. 


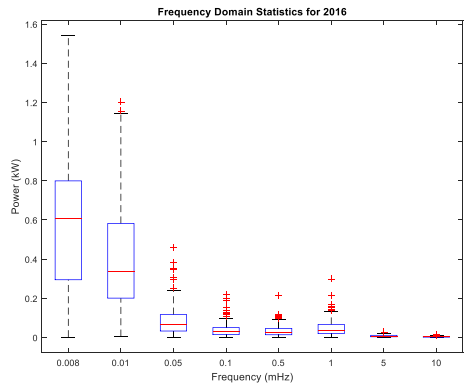

(a)

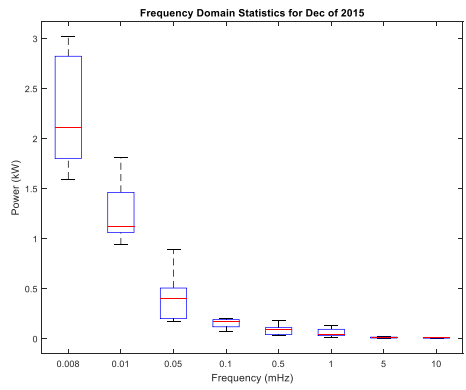

(c)

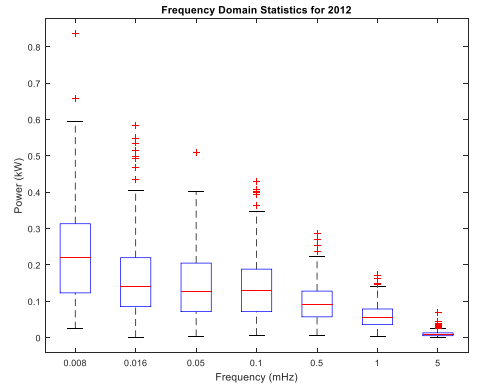

(b)

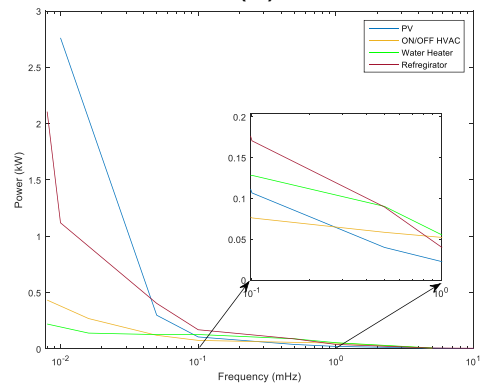

(d)

Figure 7. Boxplots illustrating the annual statistics for the spectrum of (a) on/off HVAC, (b) WH, and (c) refrigeration power consumptions and (d) compared with the one of PV.

Figure 8 compares the power consumed by a HVAC and a WH and the power generated by solar PV over 1 year. The HVAC curve shows that at $1,563 \mathrm{~h}$ in 1 year $(17.8 \%)$, HVAC demand is greater than $1.5 \mathrm{~kW}$. The WH curve shows that the WH was used for $283 \mathrm{~h}$ in that year $(3.2 \%)$. The PV curve shows that the $13 \mathrm{~kW}$ solar PV panel generates $5 \mathrm{~kW}$ ( $38 \%$ of its total capacity) for more than $1,500 \mathrm{~h}$ in 1 year. PV generation and HVAC consumption show similar behavior. Because a WH is on/off, it does not follow solar PV generation. However, by aggregating HVAC and WH loads, the solar PV signal can be closely tracked. A centralized controller will take a set of these loads and dispatch them to match solar PV generation. Details of the controller formulation found in Section 2.5.

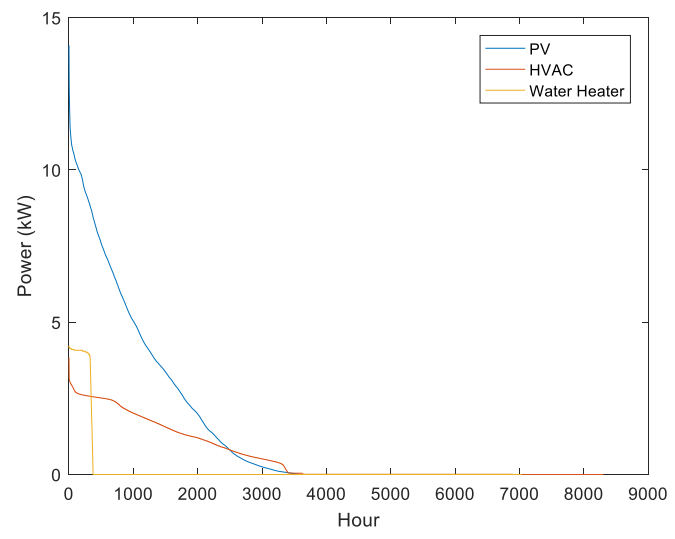

Figure 8. Comparison of duration curves for HVAC, WH, and PV powers.

\subsection{DISTRIBUTION-LEVEL PV INTEGRATION AND PENETRATION ANALYSIS}

This section presents a steady-state and quasistatic time series (QSTS) power flow analysis on the Southern Company distribution circuit that exemplifies the impact of high PV penetration on the distribution circuit. A background on distribution system voltage control is presented in Appendix A.1, while a background on coordination of building HVAC load control with smart PV inverters is presented in Appendix A.2. The team also conducted a similar QSTS power flow analysis on the Institute of 
Electrical and Electronics Engineers (IEEE) 13 Node Test Feeder, and the results are presented in Appendix A.3.

Data from two distribution feeder circuits (Feeders 1 and 2) provided by Southern Company were obtained in the format of a CymDist 5.04.12 Zipped Self-Contained Study. The data were converted to the OpenDSS data format for further study and analyses. This section initially presents power flow and LTC analyses for a feeder distribution circuit within Southern Company's power system in which three single-phase solar PV inverters that operate only in maximum power-point tracking (MPPT) mode are placed in the farthest ends of three individual phases as the worst-case scenario.

Different solar PV penetrations were investigated, and it was concluded that at a $30 \%$ penetration level, the annual accumulative LTC operation count will double as compared to the no-PV baseline case. The team further investigated the operational impacts of solar PV inverters on the same distribution feeder circuit while considering the PV inverter with constant power factor (PF) function. Feeder Circuit 1 is shown in Figure 9, and Feeder Circuit 2 is shown in Figure 10. The separated OpenDSS model for Feeder Circuit 1 is used to analyze the solar impact on the feeder.

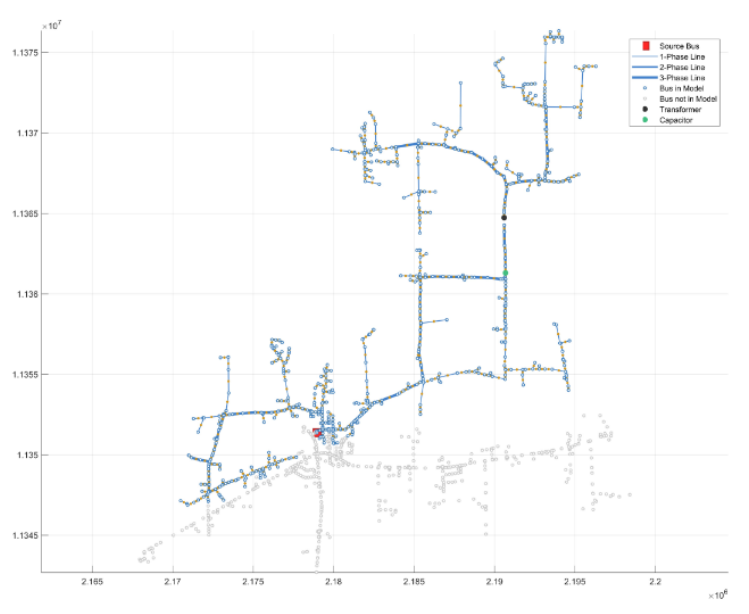

Figure 9. Feeder Circuit 1 separated using OpenDSS Tools.

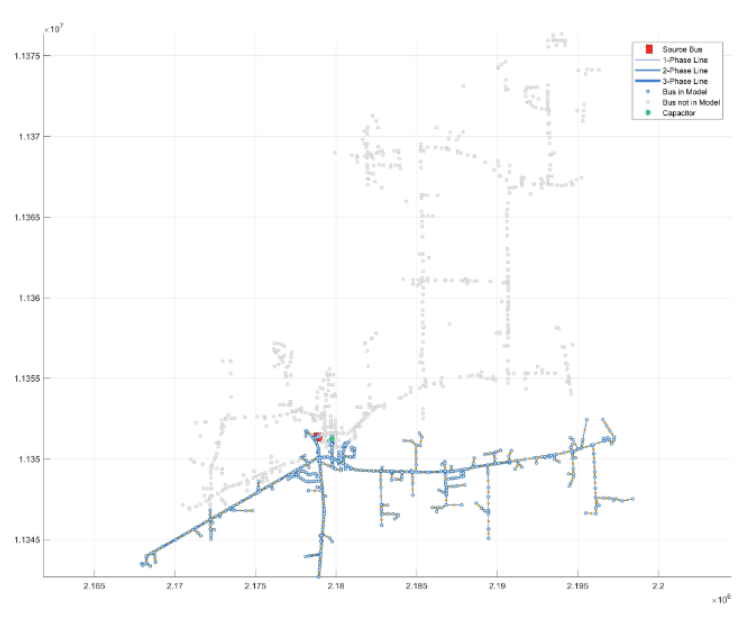

Figure 10. Feeder Circuit 2 separated using OpenDSS Tools.

Three individual single-phase feeder voltage regulation transformers are installed in the middle section of Feeder 1. Major specifications and parameters are listed as follows.

- $7.2 \mathrm{kV}, 760 \mathrm{kVA}$ per phase.

- $\quad$ LTC with 32 steps, $+/-10 \%$ and 16 steps upper, 16 steps lower.

- Potential transformer ratio is 60 .

- Regulation set point at $124 \mathrm{~V}$ with $3 \mathrm{~V}$ bandwidth.

- 45 second time delay.

- No line drop compensation.

The majority of the loads are single-phase loads with a total number of 641 in both feeders. Five large loads are three-phase loads. Load measurements were captured at 15 min intervals at both feeder circuit breakers and at the substation transformer from March 1 to May 1, 2016, and from July 1 to September 1, 2016. The daily load profiles (real power P and reactive power Q) are plotted in Figure 11 for one day on July 2, 2016. The peak loads during the data acquisition period are summarized in Table 1 . The annual 
peak load for Feeder 1 is assumed to be 2,000 kVA, which is used as the basis for the solar PV penetration level in the simulation and load tap change analyses.
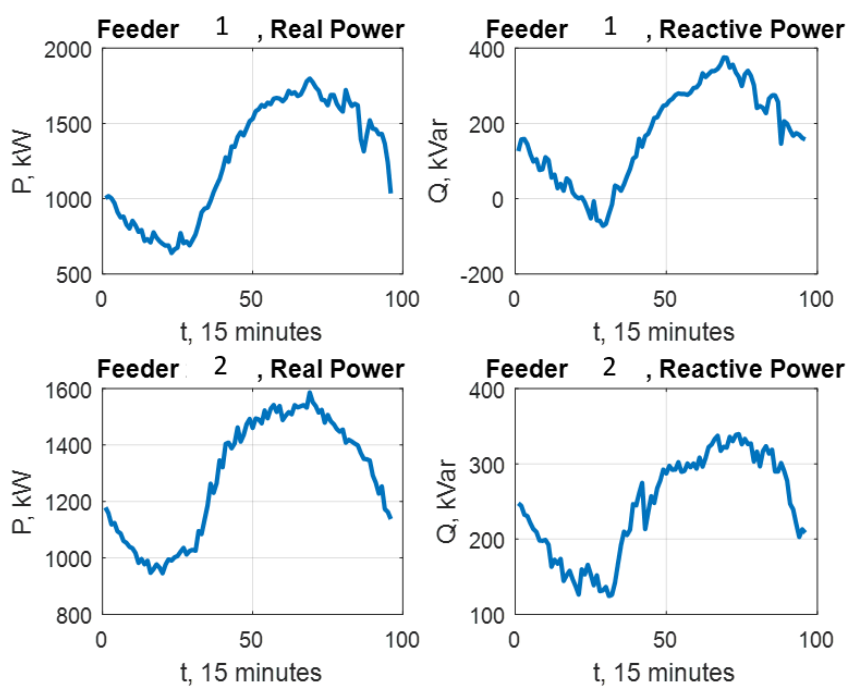

Figure 11. Feeders' daily load profiles (measurements on July 2, 2016, 00:00AM to 11:45PM).

Table 1. Peak load based on 15 min measurements from July 1 to September 1, 2016

\begin{tabular}{|c|c|c|c|c|c|c|c|c|}
\hline \multicolumn{3}{|c|}{ Feeder 1 } & \multicolumn{3}{c|}{ Feeder 2 } & \multicolumn{3}{c|}{ Substation } \\
\hline P (kW) & Q (kVar) & S (kVA) & P (kW) & Q (kVar) & S (kVA) & P (kW) & Q (kVar) & S (kVA) \\
\hline 1797.60 & 375.30 & 1836.36 & 2597.6 & 1295.20 & 2902.60 & 4905.20 & 1716.40 & 5126.23 \\
\hline
\end{tabular}

The intent is to analyze the situation when the solar PVs are installed at the very end of the feeders, which is known to be the worst-case scenario, and the associated voltage regulation problems, as depicted in Figure 12. In this study, the team focused on only one feeder, Feeder 1, since it contains an LTC transformer, i.e., voltage regulator, in the middle section of the feeder.

As aforementioned, Feeder 1 annual peak load is assumed to be $2,000 \mathrm{kVA}$ based on the fieldmeasured peak load at $1,836 \mathrm{kVA}$ during a part of the year 2016. Three single-phase solar PV inverters are placed at each phase of the feeder end and connected to the bus as listed in Table 2. Since we are investigating the LTC operations on Feeder 1, we use this feeder's annual peak load as a basis when defining solar PV penetration levels, e.g., 100\% PV penetration corresponds to 2,000 kVA solar PV installations. At the substation level, this is roughly around $30 \%$ penetration, considering peak load measurement during the summer, i.e., $5,126 \mathrm{kVA}$, and the substation transformer's rated capacity is 7,500 kVA.

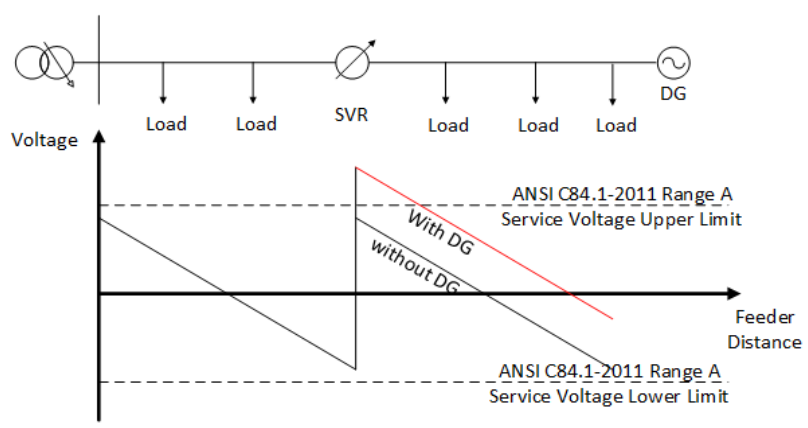

Figure 12. Distribution voltage regulation problems due to solar PV generation. 
Table 2. Solar PV inverter configurations.

\begin{tabular}{|c|c|c|c|}
\hline PV inverter & $\begin{array}{c}\text { Maximum capacity (kVA) } \\
\text { (100\% feeder penetration) }\end{array}$ & $\begin{array}{c}\text { Maximum capacity (kVA) } \\
\text { (30\% feeder penetration) }\end{array}$ & Phase \\
\hline PV1 & 667 & 200 & A \\
\hline PV2 & 667 & 200 & B \\
\hline PV3 & 667 & 200 & C \\
\hline
\end{tabular}

Snapshot power flow solutions for a typical day at noon time were derived for a baseline case (no PV), $30 \%$ PV case, and 100\% PV case, as shown in Figures 13(a)-(c). In the circuit plots, different colors denote the voltage per unit at different feeder locations, and the line thickness represents the line current. Comparing the plots, one can observe that the increased solar PV generation will create higher voltage at the feeder end where the PV is connected and the LTC transformer node.

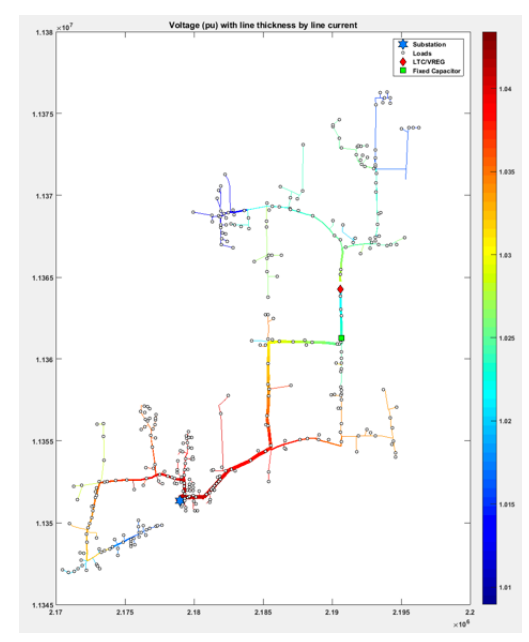

(a) Baseline case (no PV)

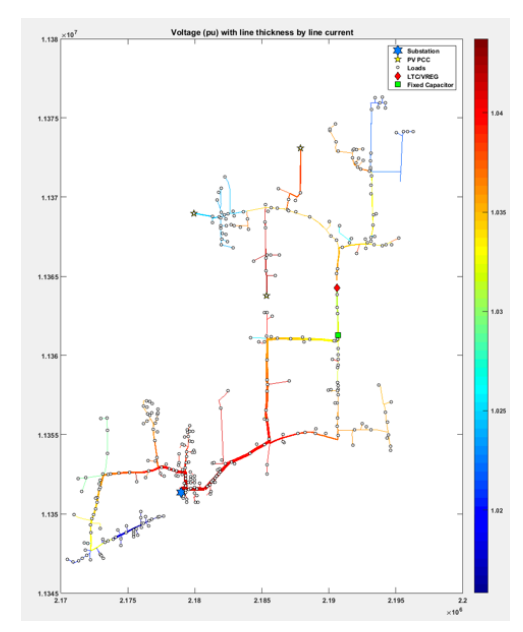

(b) $30 \%$ solar PV case

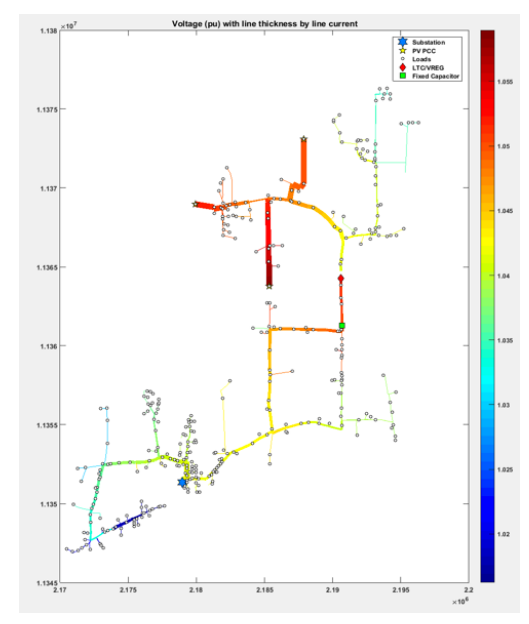

(c) $100 \%$ solar PV case

Figure 13. Snapshot power flow for Feeder 1 circuit.

The feeder voltage profiles for the same time snapshot are illustrated using spider plots as shown in Figure 14 for the baseline case (no PV), the 30\% PV case, and the 100\% PV case, which are discussed as follows:

- Baseline case (no PV): Phase A and B LTCs do not change the tap position because the voltage is within the control bandwidth, but for Phase C, the LTC has to move up to a higher position to maintain the voltage profile within the American National Standards Institute (ANSI) limit due to the heavy load in this phase and high line voltage drop.

- $\quad 30 \%$ PV case: With the same load profile, Phase A and Phase B LTCs still do not operate, but solar power generation has elevated their voltage profiles; Phase C LTC still moves up but in fewer steps.

- $\quad 100 \%$ PV case: With increasing solar power generation, both Phase A and Phase B LTCs now move down to lower positions, and Phase $\mathrm{C}$ tap moves up. This has verified the fact of increasing LTC operation due to the increased solar PV generation at one-time snapshot. The team demonstrated that 
the increased LTC operation counts over a long period of time due to the solar power fluctuations for different penetration levels.

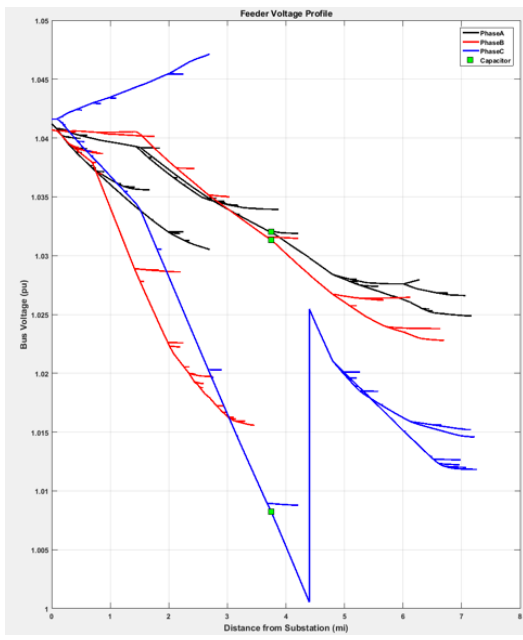

(a) Baseline case (no PV)

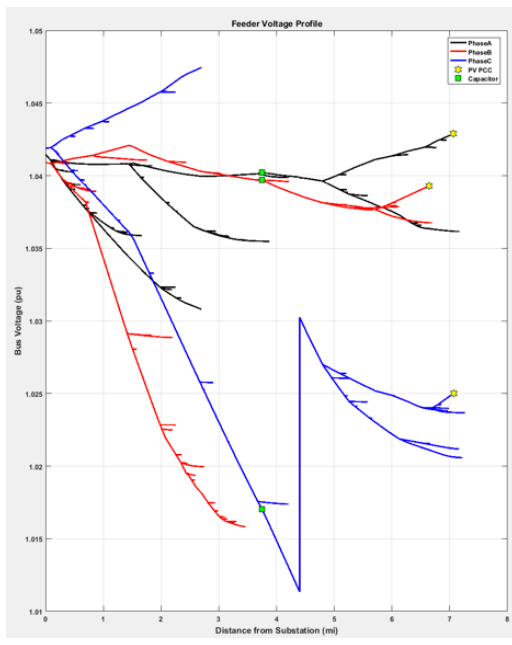

(b) $30 \%$ solar PV case

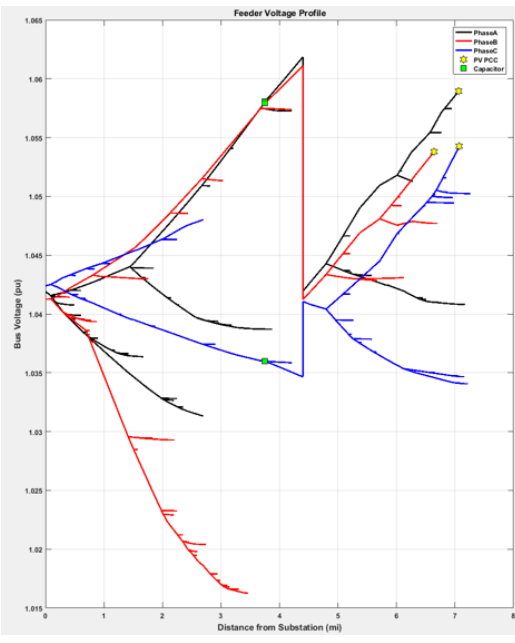

(c) $100 \%$ solar PV case

Figure 14. Feeder 1 snapshot voltage profiles (spider plots).

To closely observe the time-varying power and voltage, one day period simulations at 1 min time steps were performed by using the OpenDSS software package for the baseline case and the $100 \%$ PV penetration case for comparison. Figure 15 illustrates the power and voltage dynamics at different feeder locations, such as the feeder head, feeder end, fixed capacitor bank, and - most importantly - the LTC location. At midday, when solar power is ramping up, the feeder voltages at different locations become higher than the baseline case, and the feeder net load reduces due to the solar power generation. Figure 15(f) demonstrates significant voltage variability at the voltage regulator node during this time period, hence potentially leading to excessive tap change operations. 

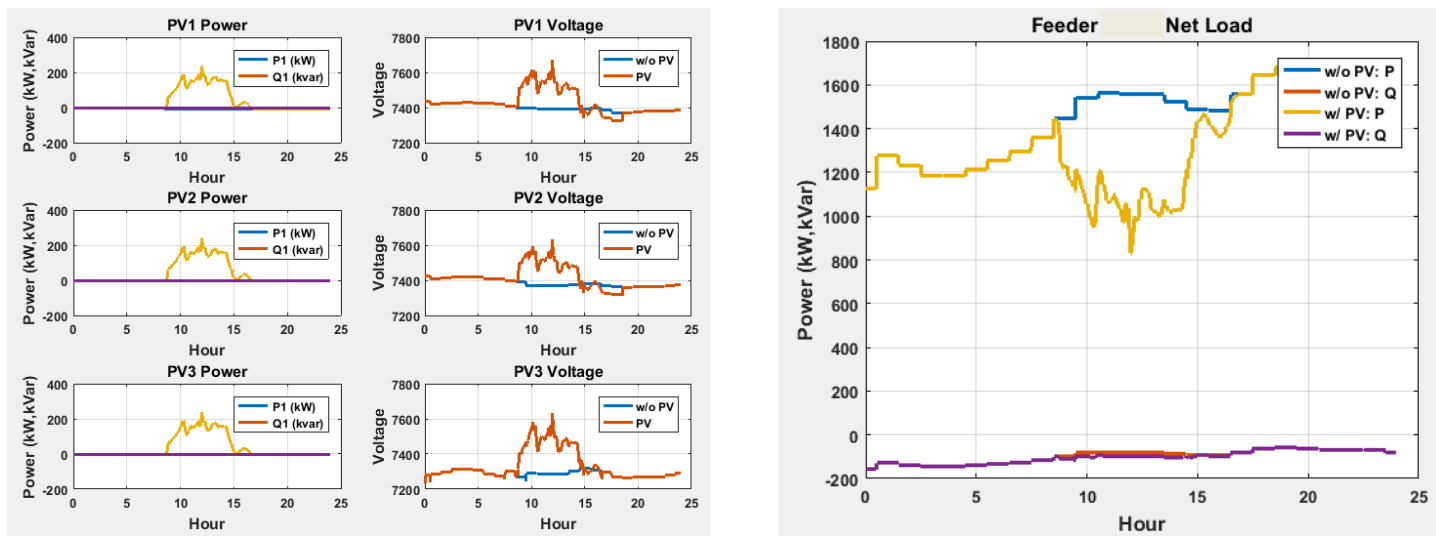

(a)

(b)
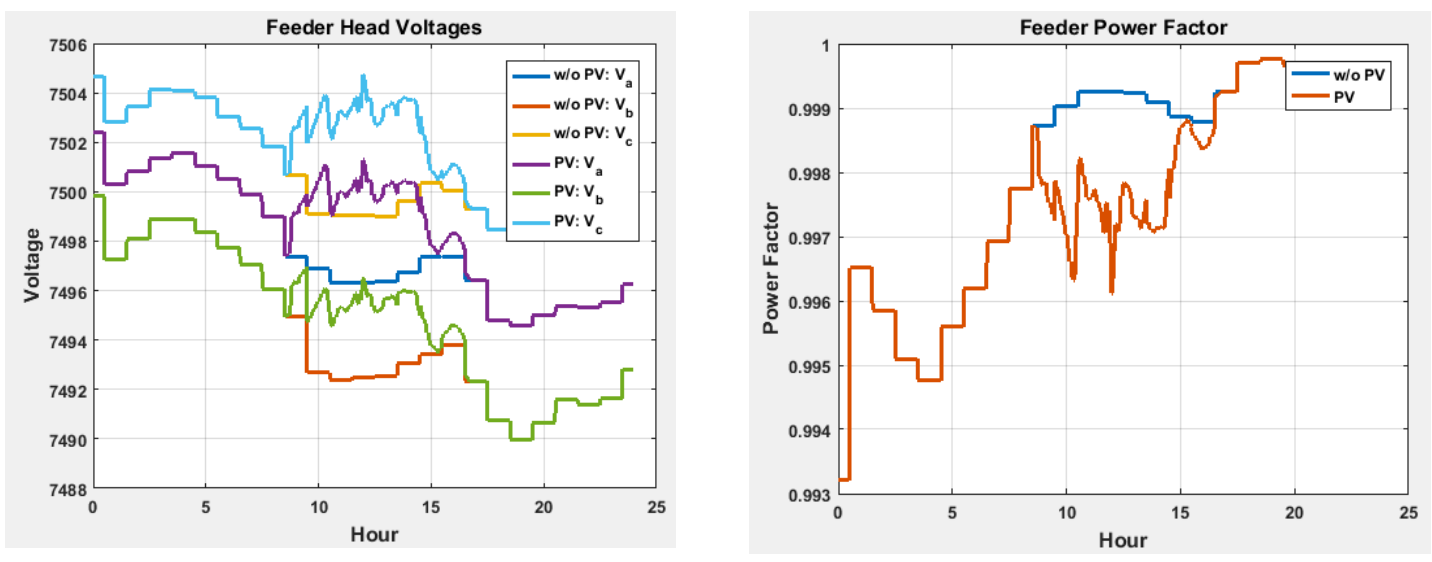

(c)

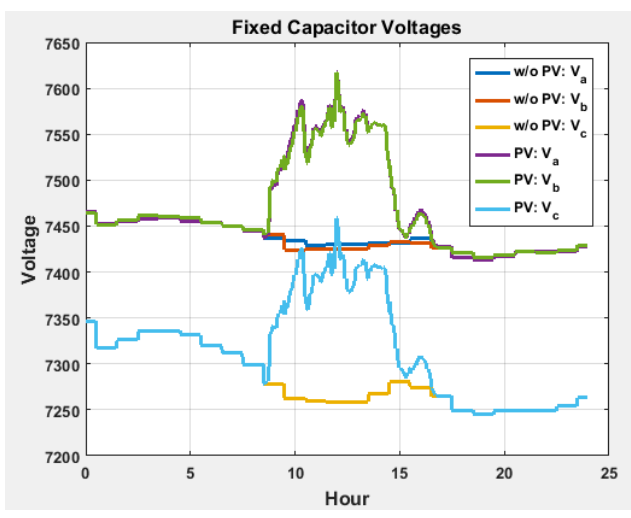

(e)

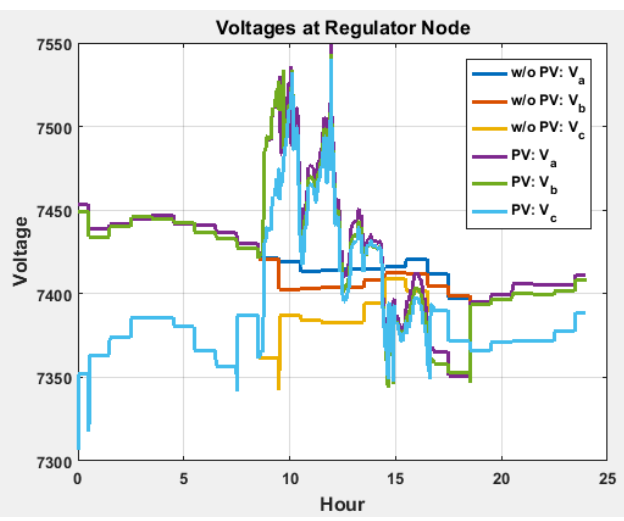

(f)

Figure 15. Power and voltage dynamics during a 1 day simulation. (a) Solar PV power generation and PV node voltage, baseline vs. 100\% PV; (b) total net load, baseline vs. 100\% PV; (c) head voltage, baseline vs. 100\% PV; (d) PF, baseline vs. 100\% PV; (e) cap bank node voltage, baseline vs. $100 \%$ PV; and (f) LTC transformer downstream-side node voltage, baseline vs. 100\% PV. 
To investigate the potential effects on LTCs, the OpenDSS simulations for a 1 week time period at a 1 min time step were run for the baseline case and 30\% PV penetration case. The voltage regulator load tap positions $(-16$ to +16$)$ for a 1 week period for baseline vs. $30 \%$ PV are plotted in Figure 16. Significant increases of tap operations are shown. The cumulative tap changes are from 1 to 6 changes for the Phase A tap, 1 to 8 changes for the Phase B tap, and 20 to 37 changes for the Phase C tap.

Figure 17 details the solar power variations and voltage fluctuations for the individual phases. When the solar power is ramping up, the voltage at the regulator node increases and might exceed the LTC control bandwidth, that is $(124 \mathrm{~V}+/-1.5 \mathrm{~V}) \times 60$. When the voltage
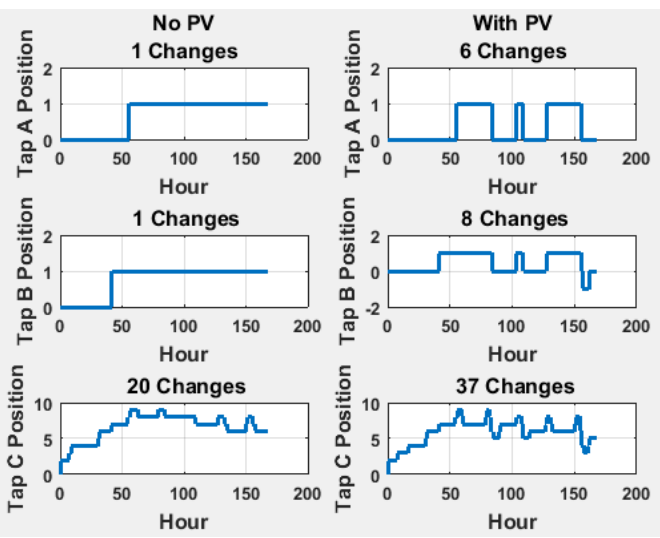

Figure 16. Voltage regulator LTC positions over a 1 week period for baseline vs. $30 \% \mathrm{PV}$. increases or decreases beyond the upper or lower bandwidth, the LTC will be enabled after a $45 \mathrm{~s}$ time delay. The load tap may move up or down several steps to bring the feeder voltage back within the limits.

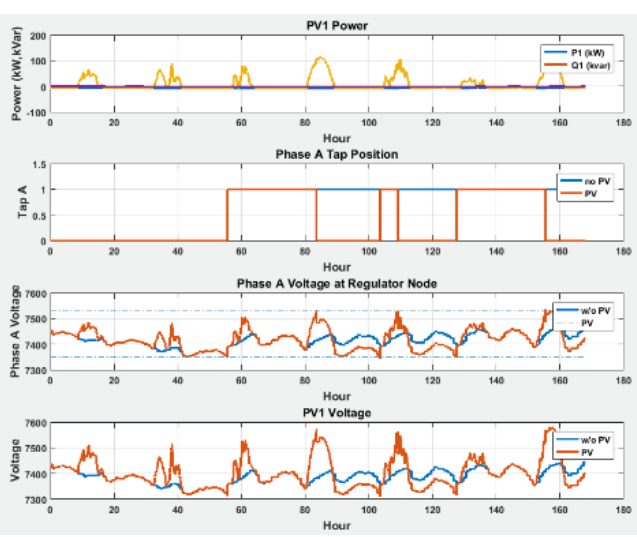

(a)

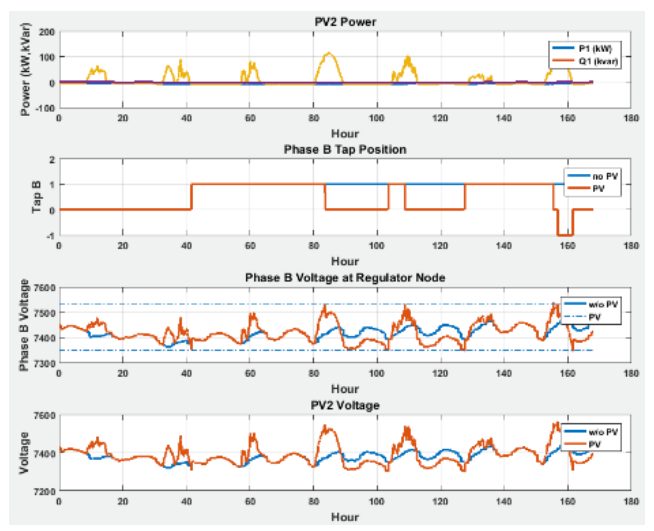

(b)

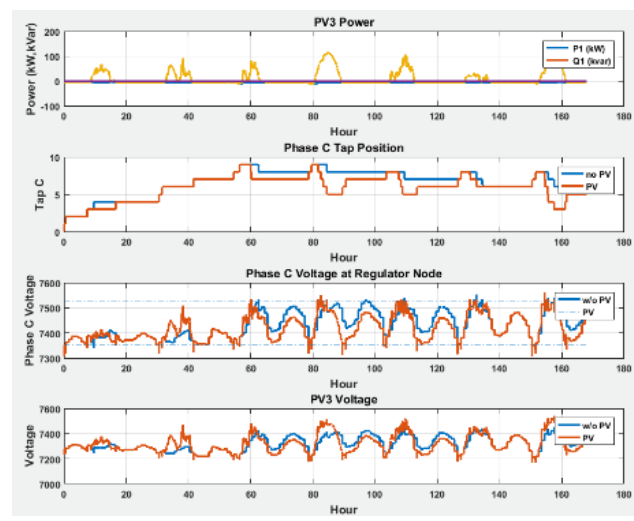

(c)

Figure 17. Voltage fluctuations and load tap changes due to solar power ramping for baseline vs. 30\% PV. (a) Phase A, (b) Phase B, (c) Phase C. 
The accumulation of load tap changes over a 1 week time period for the baseline case and various PV penetration levels from $10 \%$ up to $150 \%$ is plotted in Figure 18. At 10\% PV penetration, the load tap changes increase by $23 \%$; at $20 \%$ PV penetration, the load tap changes increase by $59 \%$. Substantial load tap changes increase will occur for further increases in PV penetration levels.

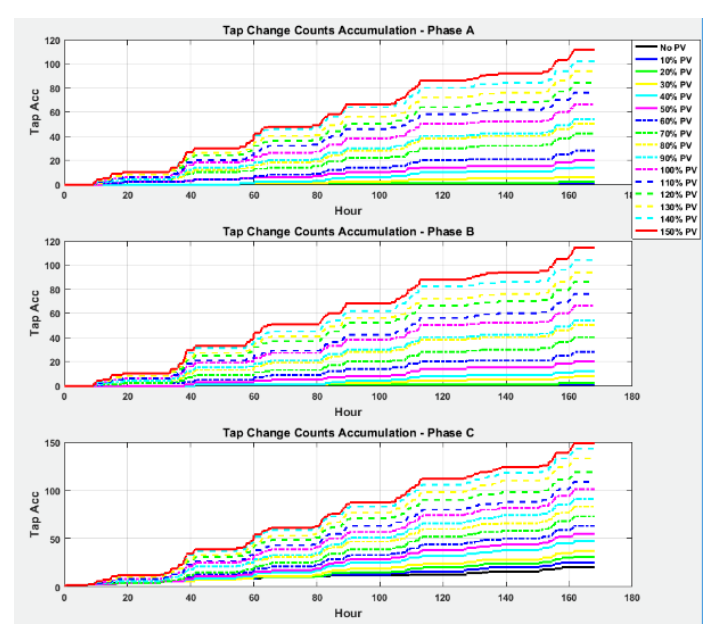

(a)

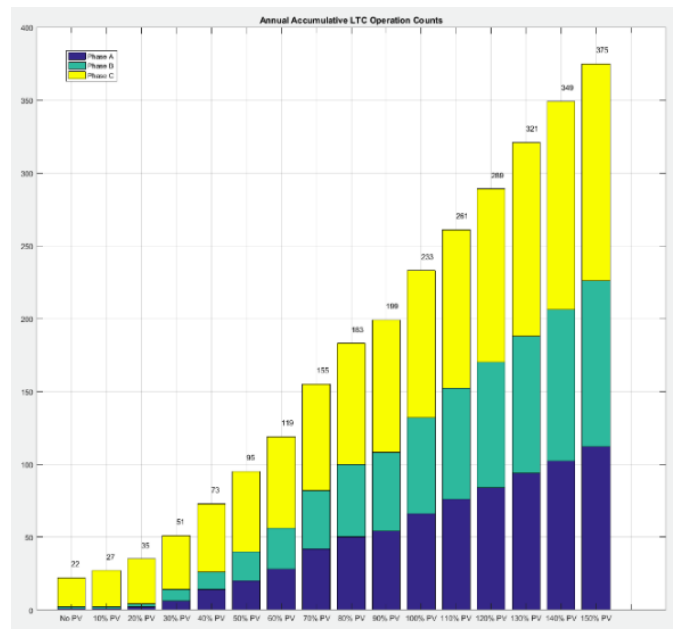

(b)

Figure 18. Cumulative LTC operation counts for different PV penetrations. (a) Load tap changes accumulation over 1 week and (b) total operation counts in 1 week.

To further validate the effects of different PV penetration levels on the LTC operations, simulations of the whole 1 year time period were conducted at a 1 min time step for 16 different scenarios, including the baseline case and the PV penetration from 10 to $150 \%$. The tap change operation count accumulation is shown in Figure 19. The simulations show that at less than 20\% PV penetration, the cumulative load tap changes are roughly the same; at 30\% PV penetration, the cumulative load tap change counts are doubled compared with the baseline case and then almost linearly increase with the increasing PV penetration levels at a rate of about 1,500-1,600 counts every $10 \%$ penetration increase.

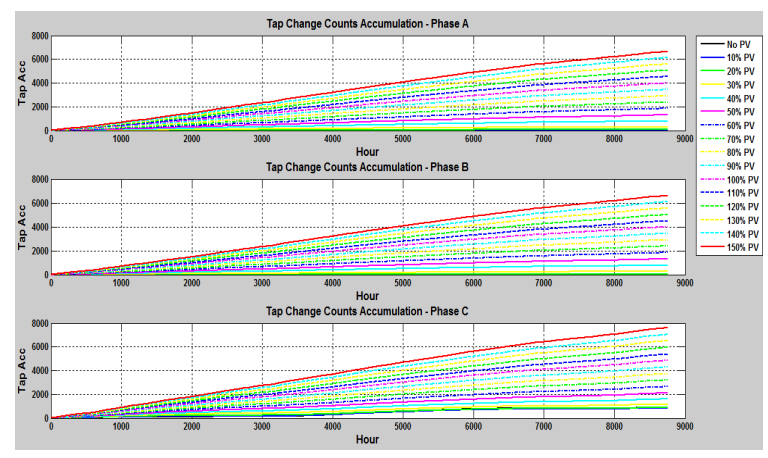

(a)

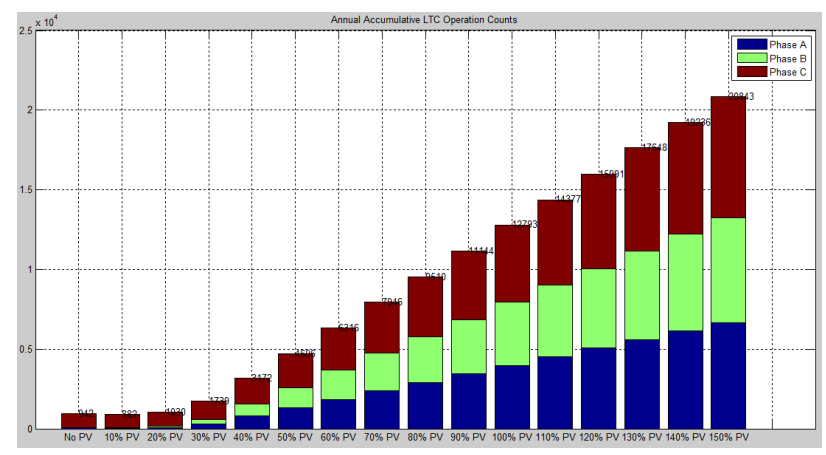

(b)

Figure 19. Cumulative LTC operation counts for different PV penetrations. (a) Total operation counts in 1 year and (b) load tap changes accumulation over a 1 year period.

Then, the team further investigated the operational impacts of solar PV inverters on the same feeder distribution circuit, but they amended the PV inverter operation from only MPPT mode to constant PF mode by using the OpenDSS solar PV inverter element, as demonstrated in Figure 20, although one snapshot of the feeder circuit voltage (by color) and current (by line thickness) is illustrated in Figure 21. 
The steady-state power flow analyses were performed in OpenDSS for the baseline (no PV), 30\% PV with PV inverter on MPPT mode only, and 30\% PV with PV inverter on MPPT and fixed PF at 0.90 (leading or lagging). When the PV inverter contributes reactive power, the active power will be limited due to the fixed or rated hardware capacity, so the MPPT will only be valid for the active power below the limit needed for reactive power compensation. Figure 22 illustrates the feeder-distance voltage profile for the three cases, and it is observed that the feeder voltage will be further elevated if the reactive power function is enabled.

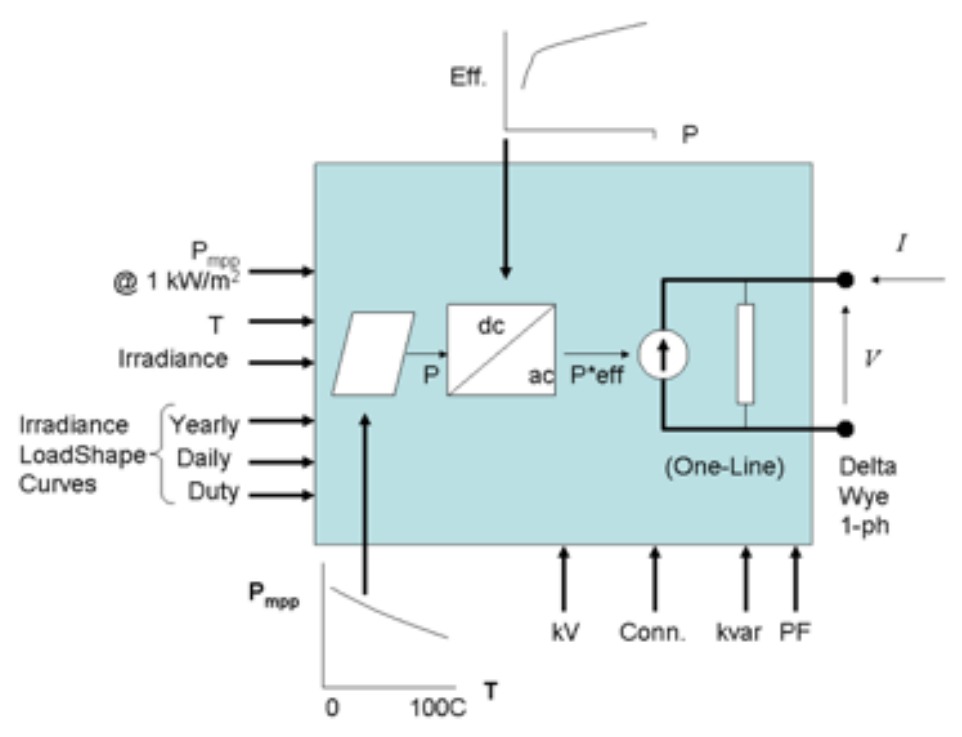

Figure 20. Solar PV inverter model in OpenDSS [157].

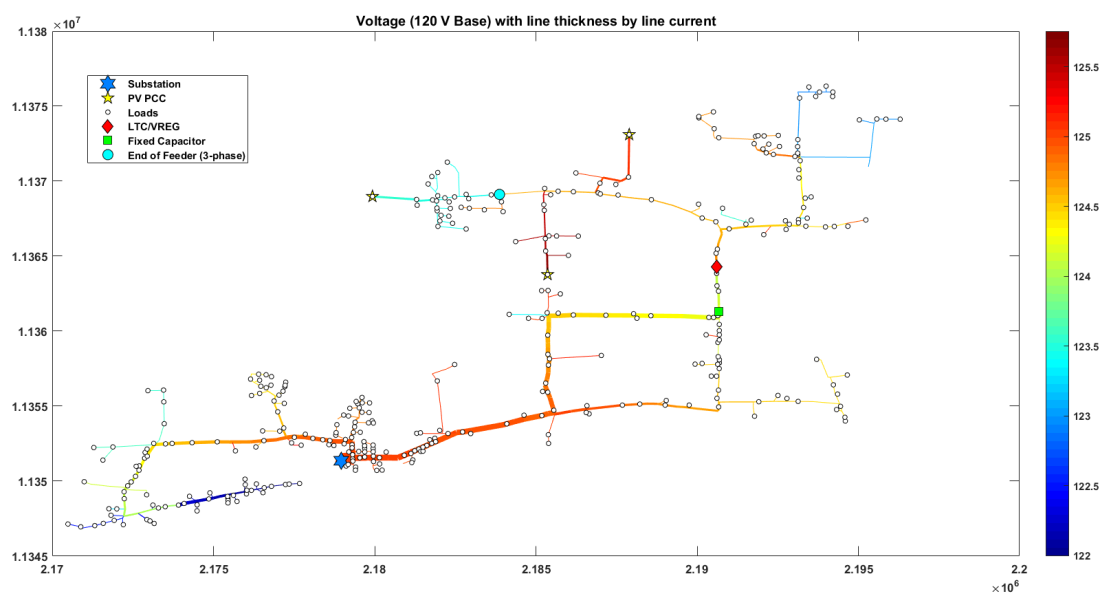

Figure 21. The Southern Company's feeder circuit under investigation (30\% penetration by capacity). 


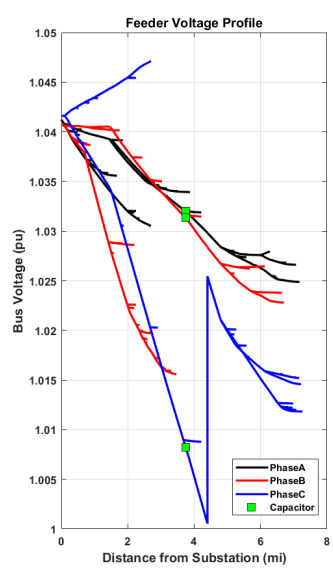

(a)

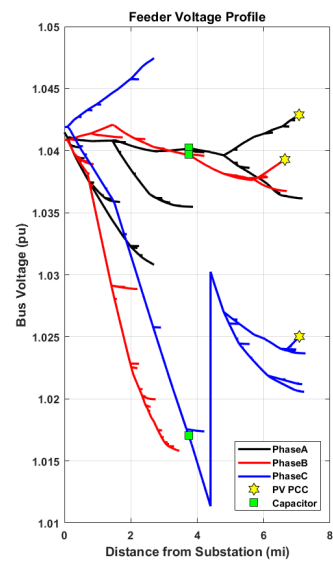

(b)

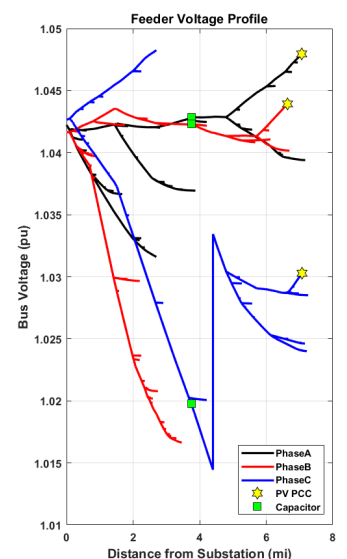

(c)

Figure 22. Feeder-distance voltage profile for the three cases under investigation. (a) No PV, (b) MPPT only, and (c) with reactive power $(\mathrm{PF}=0.9)$.

For the LTC operation impacts, the team analyzed the cumulative operation counts of the LTCs associated with the feeder voltage regulators. The results are illustrated in Figure 23 for a 1 week period. The LTC operation count was further increased with a solar PV inverter with a fixed PF function. This also concludes that without proper coordination between solar PV inverters and other voltage control devices, the operation impact tends to deteriorate. The team explored the efficacy of co-optimizing the load modulation/control schemes along with smart inverter control schemes to achieve two key objectives: absorb the PV powers and optimize the power flow.
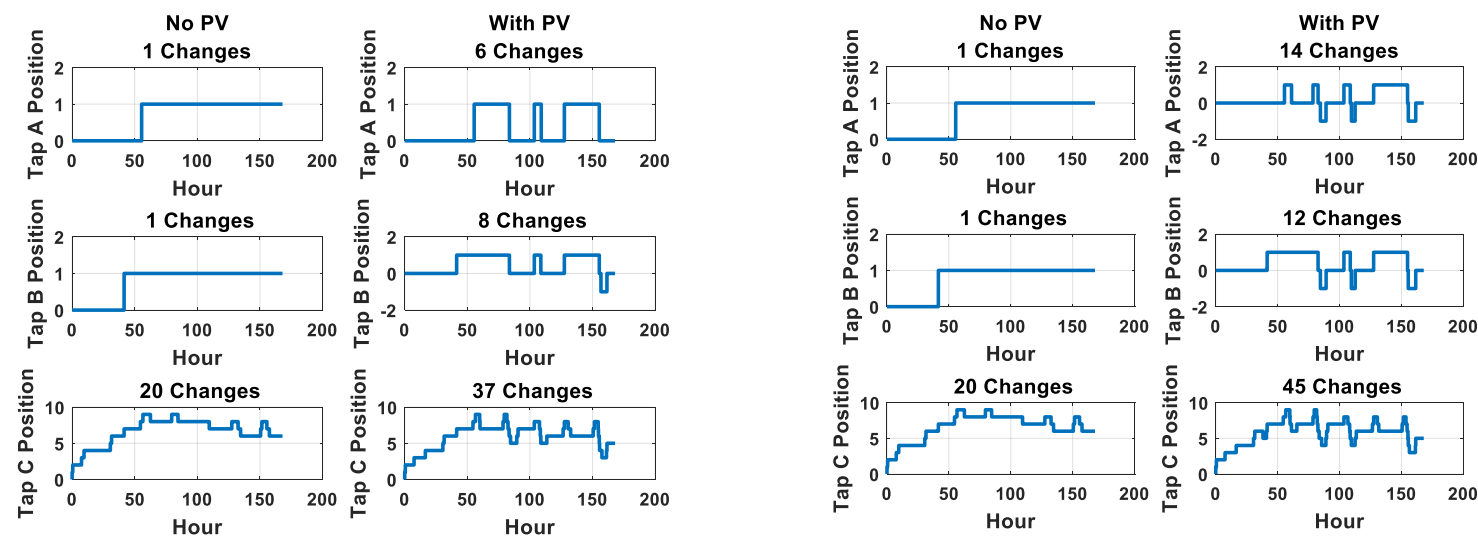

(a)

(b)

Figure 23. LTC cumulative operations for 1 week (30\% percent PV by capacity). (a) PV inverter with MPPT only and (b) PV inverter with MPPT and PF $=0.9$.

To meet the first objective, the installed capacity of the PV was increased in comparison with peak load on a given segment by using load control to absorb most of the energy generated by the PV. To meet the second objective, a coordinated supervisory control of smart inverters is performed in a given segment to optimize power flow in the distribution circuit to operate in conjunction with the existing distribution voltage control assets. An updated architecture for an end-to-end system will be tested in a simulation and on a test bed for evaluating performance for improving voltage stability within distribution circuits with high penetration of solar PVs. 


\subsection{DISTRIBUTED VOLTAGE CONTROL}

This section presents a distributed optimal control mechanism for PV inverters to regulate the voltage and reduce network losses. The coordination and optimal control of PV inverters in the proposed control mechanism are achieved in a distributed manner by solving a small local optimization problem for each PV with limited information exchange.

Increasing the penetration of variable generation sources such as solar PVs leads to more frequent voltage fluctuations in a distribution power grid [44-45]. The PV inverters can provide fast and flexible voltage regulation by controlling the active and reactive power output in real time [46]. Traditionally, the distribution system operator (DSO) optimizes the control and operation of voltage regulation and reactive power compensation devices in the distribution network by solving a centralized optimal power flow (OPF) problem, known as the integrated volt-var controller (IVVC) [47-49], in a distribution management system (DMS). However, the centralized method faces the following challenges when many PV inverters are integrated into the distribution network.

- The complexity and size of a centralized optimization problem increase significantly with the number of PV inverters, making it difficult to solve. The computational time might not satisfy the requirements for mitigating fast-changing solar power generations.

- The centralized methods need communication connections between the DSO and every PV inverter. The volume of data flow over the communication network will increase dramatically and could lead to a communication bottleneck.

- Centralized control might not be resilient to incidents. When the DMS is down, the whole system will fail. This in known as a single point of failure.

To address these challenges, the team developed a distributed optimal voltage control method for PV inverters that is efficient to solve and only requires limited communications. Each PV inverter will optimize its own active and reactive power output by solving a local optimization problem based on its own and neighbors' information. Through limited information exchange among the PV inverters, the distributed optimization algorithm can achieve the global optimum within a few iterations. In this work, the problem was first formulated as a second-order cone programming (SOCP)-based centralized optimization model for voltage regulation and loss minimization in distribution networks. Then, the centralized optimization model was decomposed into small local optimization problems for each PV inverter. The reformulated problems were solved in a distributed manner by using the alternating direction method of multipliers (ADMM) [50-52]. The ADMM is a promising distributed algorithm that has fast and stable convergence. The convexity of the developed model guarantees the convergence of the distributed algorithm and the global optimum. Case studies are provided in which the developed ADMMbased distributed optimal PV control method is validated by being compared with the centralized method. The efficacy of the proposed method is demonstrated through OpenDSS [53] simulations.

The team formulated a centralized optimization model for PV control based on the SOCP-based branch flow model [54] of distribution networks. The objectives are to minimize the total nodal voltage deviation and network power loss as:

$$
\min \left(\varpi_{1} \sum\left(u_{i}-2 v_{i} u_{0}+u_{0}^{2}\right)+\varpi_{2} \sum r_{i j} l_{i j}\right)
$$

so that 


$$
\begin{gathered}
\left\|2 v_{i} \quad u_{i}-u_{0}\right\|_{2} \leq u_{i}+u_{0}, \\
\left\|2 P_{i j} \quad 2 Q_{i j} \quad l_{i j}-u_{i}\right\|_{2} \leq l_{i j}+u_{i}, \\
P_{i j}-l_{i j} r_{i j}-\sum_{k \in v(j)} P_{j k}+P_{P V, j}+P_{G, j}-P_{D, j}=0, \\
Q_{i j}-l_{i j} x_{i j}-\sum_{k \in v(j)} Q_{j k}+Q_{P V, j}+Q_{G, j}-Q_{D, j}=0, \\
u_{j}=u_{i}-2\left(r_{i j} P_{i j}+x_{i j} Q_{i j}\right)+\left(r_{i j}^{2}+x_{i j}^{2}\right) l_{i j}, \\
\left\|P_{P V, j} \quad Q_{P V, j}\right\|_{2} \leq S_{P V, j}, \\
\left\|P_{G, i} \quad Q_{G, i}\right\|_{2} \leq S_{G, i}, \\
0 \leq P_{P V, j} \leq P_{P V, j}^{\max }, \\
\left(V_{i}^{\min }\right)^{2} \leq u_{i} \leq\left(V_{i}^{\max }\right)^{2}, \\
0 \leq l_{i j} \leq l_{i j}^{\max },
\end{gathered}
$$

where $u_{i}$ is the square of the voltage of node $i ; l_{i j}$ is the square of the current in line $i j ; u_{0}$ is the square of nominal voltage, which is 1.0 p.u.; $v_{i}$ is the nodal voltage; $P_{i j}$ and $Q_{i j}$ are the active and reactive power in line $i j ; P_{G, i}$ and $Q_{G, i}$ are the active and reactive power injections at the substation node; $S_{G, i}$ is the capacity of the substation; $S_{P V, j}, P_{P V, j}$ and $Q_{P V, j}$ are the capacity and active and reactive power output of the PV at node $j$; and $P_{D, j}$ and $Q_{D, j}$ are the active and reactive power demand at node $j$.

Equation (1) is the objective function with two terms representing the total nodal voltage deviations and network power loss, respectively, and $\varpi_{1} \varpi_{2}$ are the weighting factors of the two objectives. The balance or trade-off between the two objectives can be adjusted by setting the weighting factors. Equation (2) represents the relationship between $u$ and $v$ in a relaxed convex form. Equation (3) is the relaxed SOCP constraint that represents the relationship between active power, reactive power, voltage, and line current. Equations (4) and (5) are the nodal active and reactive power balance equations. Equation (6) indicates the voltage drop between the two end nodes of a distribution line. Equations (7) and (8) represent the capacity of PV inverters and the substation, respectively. Equation (9) is the maximum active power generation limit of the PV inverter. Equation (10) is the nodal voltage limit. Equation (11) is the line current limit. This SOCP-based model is a strictly convex optimization model. The proposed model is a convex model that contains some convex relaxations with exactness [55].

Next, the team formulated the general form consensus optimization [56] for optimal voltage control of PV inverters in distribution systems based on ADMM as follows: 


$$
\min \mathrm{L}_{\rho}(x, z, \lambda)=\sum_{i=1}^{N}\left[f_{i}\left(x_{i}\right)+\lambda_{i}^{T}\left(\tilde{x}_{i}-z_{i}\right)+(\rho / 2)\left\|\tilde{x}_{i}-z_{i}\right\|_{2}^{2}\right]
$$

where $\lambda^{T}$ is the transpose of Lagrangian multiplier, and $\rho$ is the iteration step size. Equation (12) is the augmented Lagrangian function of the objective function in Eq. (1), where $f_{i}\left(x_{i}\right)$ is the same with the objective function in Eq. (1).

$$
\begin{gathered}
x_{i}^{k+1}=\underset{x}{\arg \min }\left(f_{i}\left(x_{i}\right)+\left(\lambda_{i}^{k}\right)^{T} x_{i}+(\rho / 2)\left\|\tilde{x}_{i}-z_{i}^{k}\right\|_{2}^{2}\right), \\
z_{g}^{k+1}=\left(1 / k_{g}\right) \sum_{G(i, j)=g}\left(\tilde{x}_{i}^{k+1}\right), \\
\lambda_{i}^{k+1}=\lambda_{i}^{k}+\rho\left(\tilde{x}_{i}^{k+1}-z_{i}^{k}\right) .
\end{gathered}
$$

Equations (13)-(15) are the iterations in ADMM, and $k$ is the iteration number. Equation (13) is the optimization model of each PV; $\arg$ min indicates the values of $x_{i}$, which makes the objective function reach minimum value. Equation (14) updates the global information, where $k_{g}$ is the number of coupling variables in association with the global variable, and $G(i, j)=g$ is the function of the global variable and local variables. Equation (15) updates the Lagrangian multiplier. The following criteria in Eq. (16) will be applied to check the convergence:

$$
\begin{aligned}
& \left\|s^{k+1}\right\|_{2}^{2}=\left\|\tilde{x}_{i}^{k+1}-z_{i}^{k+1}\right\|_{2}^{2} \leq \varepsilon_{1} \\
& \left\|r^{k+1}\right\|_{2}^{2}=\left\|(-\rho)\left(z_{i}^{k+1}-z_{i}^{k}\right)\right\|_{2}^{2} \leq \varepsilon_{2} .
\end{aligned}
$$

Equation (16) indicates that the 2-norm of dual residual $s$ and primal residual $r$ are less than the preset thresholds $\varepsilon_{1}$ and $\varepsilon_{2}$. The dual residual is the difference between the coupling variable and the global variable at each iteration. The primal residual is the difference between the values of the global variable at iteration $k$ and the previous iteration $k$-1. The smaller difference indicates that the solution is approaching the global optimum. The whole distribution network is partitioned into several subsystems for PVs. Taking Figure 24 as an example, the system is partitioned into two subsystems: S1 and S2.
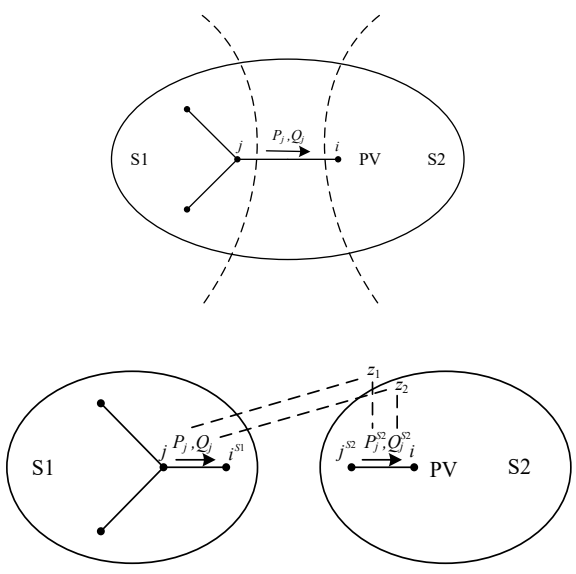

Figure 24. Subsystems in a distribution network with PVs. 
The boundary nodes are $i$ and $j$, and the boundary line is $l_{i j}$. Each subsystem has a local copy of global variables. The global variables will guarantee the consensus between the two subsystems. The information exchange between the two subsystems is shown in Figure 25. The flow chart of the ADMMbased distributed optimization algorithm is shown in Figure 26.

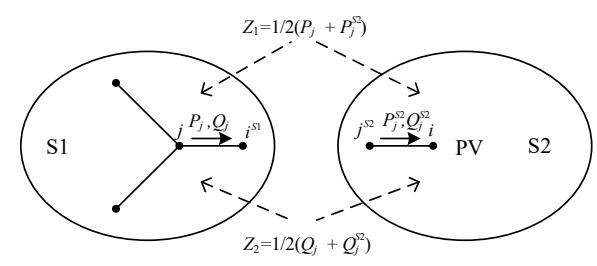

Figure 25. Information exchange between two subsystems.

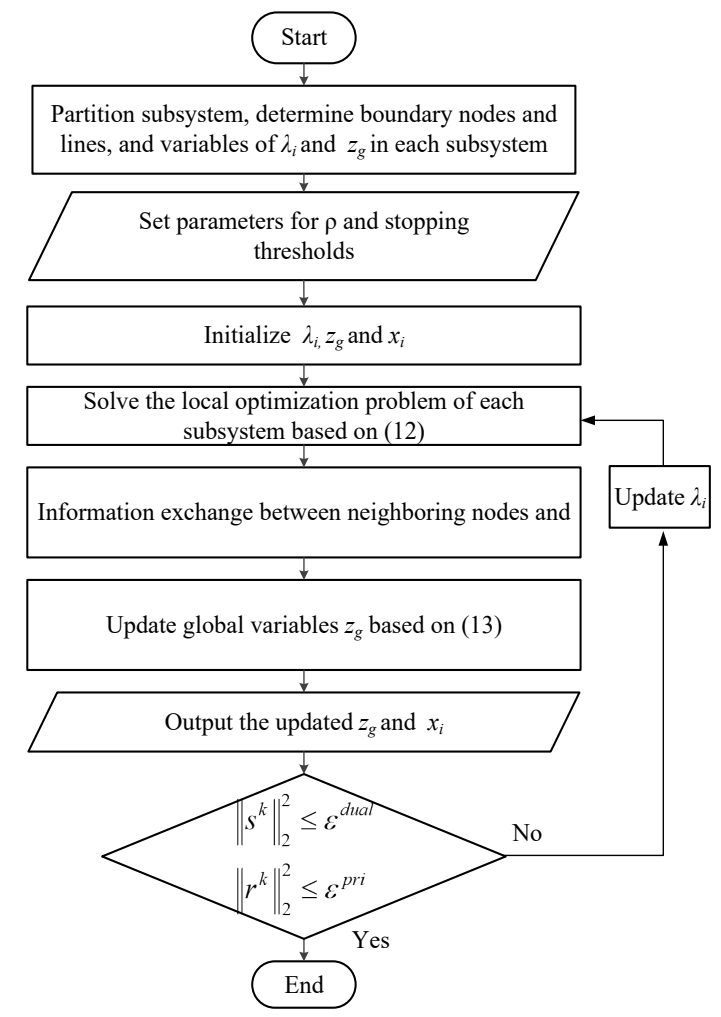

Figure 26. Flow chart of ADMM-based distributed optimal control of PVs.

Then, the team presented the implementation, simulation, and case studies of the proposed method. The proposed model was implemented in MATLAB and solved by using CPLEX [57] on a personal computer. The optimization results of the distributed algorithm (i.e., the active and reactive power output of the PV inverters) will be passed to OpenDSS as input for distribution system simulations to study the effectiveness of the proposed method. A modified IEEE 33 node case [58] with PVs was used for case studies.

\subsubsection{Single-Period Case Study}

In this case study, the number of PVs is 15. The modified case is shown in Figure 27. The ADMM algorithm settings are as follows: step size is $\rho=10$ and dual and primal residue thresholds $\varepsilon_{1}$ and $\varepsilon_{2}$ are set to 0.00005 and 0.01 , respectively. 


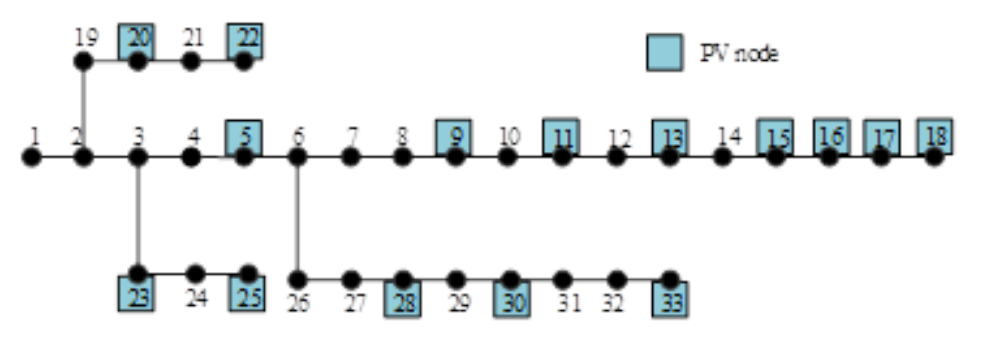

Figure 27. Modified IEEE 33 node case with 15 PVs.

The following two cases are designed to demonstrate the effectiveness of the proposed method in reducing nodal voltage deviations and network losses. The basic constant PF control of PVs is used as a benchmark in Case 1.

- $\quad$ Case 1: Use OpenDSS to simulate the constant PF control $(\mathrm{PF}=0.95)$ of PVs.

- Case 2: Use the proposed distributed PV control model to obtain the optimal results of PV outputs and pass to OpenDSS for simulation.

The proposed ADMM-based distributed optimization algorithm converges after about eight iterations. The simulation results are presented in Figure 28. The voltage profile in Case 2 is closer to 1.0, which is the nominal voltage. Additionally, the line loss in Case 2 is much lower than in Case 1. The comparison results show that the proposed method can effectively reduce the voltage deviations and network loss.

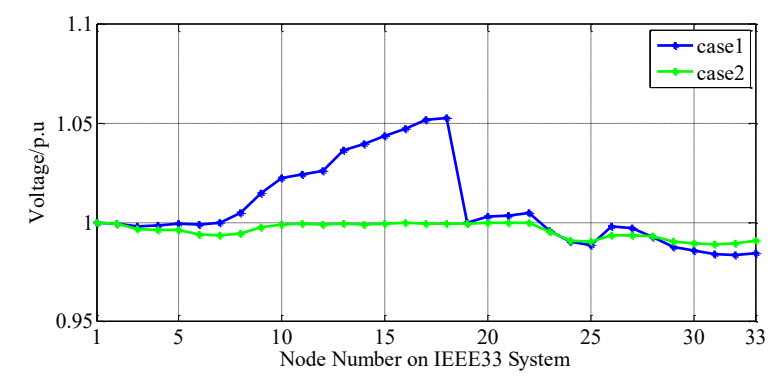

(a)

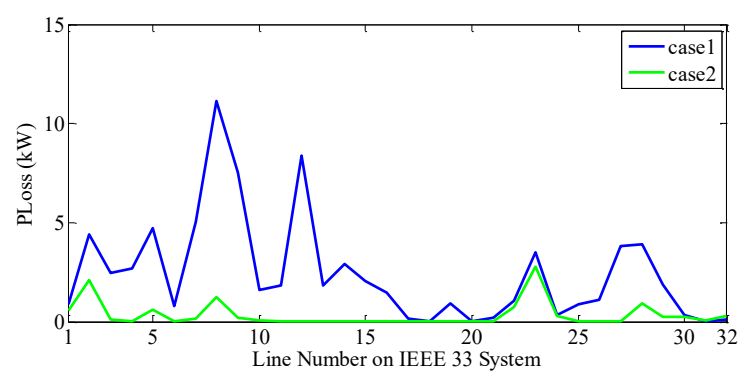

(b)

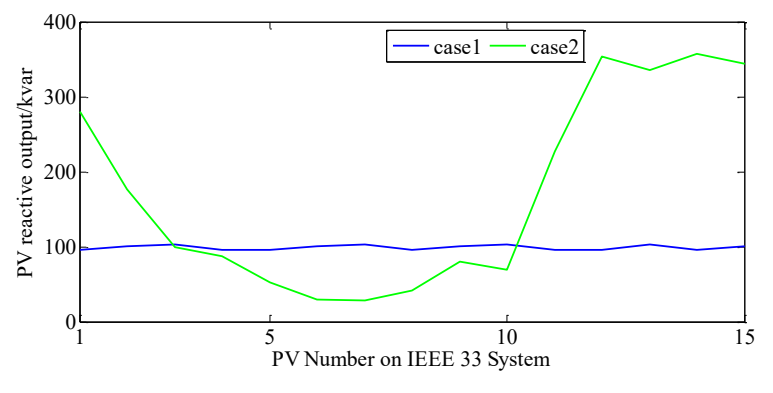

(c)

Figure 28. Comparison of (a) nodal voltages, (b) network losses, and (c) reactive power output of PVs. 


\subsubsection{Time-Series Simulation}

To mimic the online applications, the proposed distributed algorithm runs every minute to control the PV inverters for voltage regulation and loss reduction. As shown in Figure 29, for every $1 \mathrm{~min}$, an ADMMbased distributed optimal PV control algorithm is implemented to optimize the active/reactive power output of the PVs to minimize the network losses while respecting the voltage limits. Then the results are passed to OpenDSS for simulation.

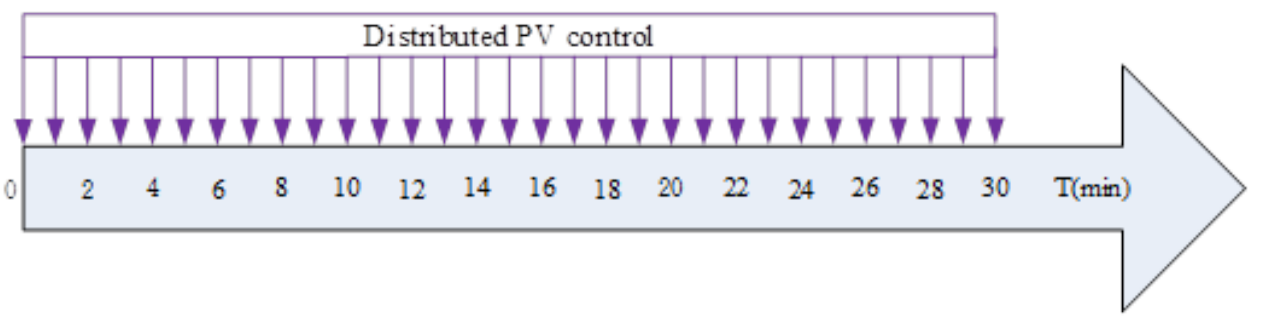

Figure 29. Time-series distributed optimal PV control in distribution networks.

A modified IEEE 33 node case with 3 PVs: The maximum power output of PV is set to $50 \%$ of the nodal load. The voltage range is set to $[0.95,1.05]$ p.u. ADMM parameters: step size $\rho=14$, and stopping criteria: $\varepsilon_{1}$ and $\varepsilon_{2}$ are 0.001 and 0.04 , respectively. Simulation time period: from 11 a.m. to 3 p.m. with 1 min resolution. Simulation software: MATLAB + OpenDSS.

The following two cases were studied:

- $\quad$ Case 1: Basic PV control with constant PF 0.95 (OpenDSS) and

- Case 2: Distributed optimal PV control (MATLAB + OpenDSS).

The simulation results (Figures 30-35) demonstrate that the proposed distributed algorithm can be implemented in online applications for real-time mitigations of voltage deviations (Figures 32-35) and reductions of power losses (Figure 31).

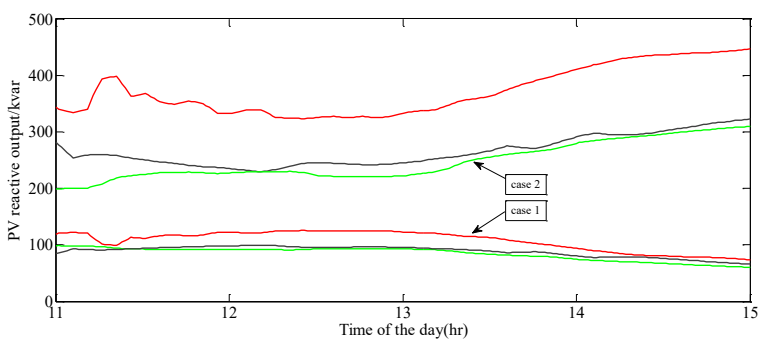

Figure 30. Reactive power output of PVs.

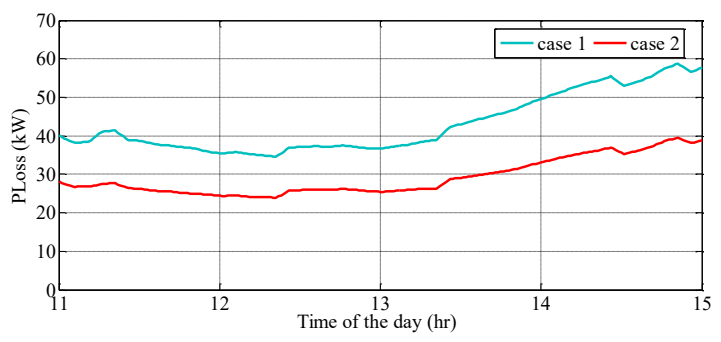

Figure 31. Total network power loss. 


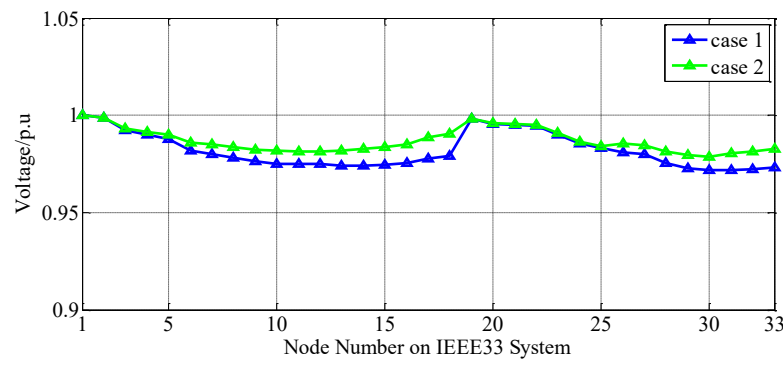

Figure 32. Nodal voltage at 12:00 p.m.

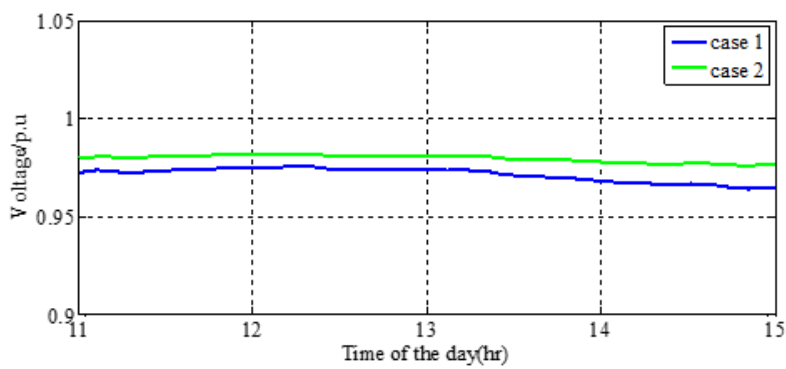

Figure 34. Voltage at node 11.

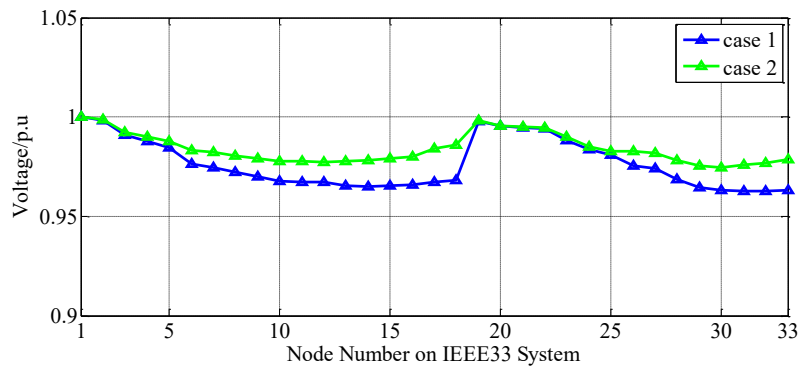

Figure 33. Nodal voltage at 2:00 p.m.

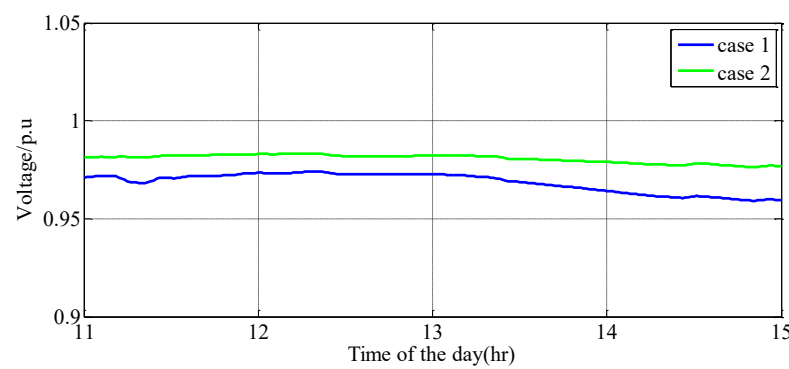

Figure 35. Voltage at node 33.

From this case study, it can be concluded that the proposed ADMM-based distributed PV control algorithm can be implemented for online applications of real-time control and optimization. It can effectively improve the voltage profile and reduce power losses.

In summary, this work developed an ADMM-based distributed optimal control method for PV inverters for voltage regulations and network loss reductions. Through the proposed method, the coordinated and optimal control of PV inverters was achieved in a distributed manner by solving a small local optimization problem for each PV with limited information exchange. The case study demonstrated that the proposed method converges to the global optimal solution in comparison with the centralized optimization model. Additionally, the simulation results demonstrated the effectiveness of the proposed method in improving the voltage profile and reducing network power loss through a comparative study by using OpenDSS simulations. The integration of large amounts of distributed PVs poses great challenges to the grid operation. The developed distributed optimization and control framework provides a promising solution, and the proposed distributed optimal PV control method has great potential in the practical applications of coordinated PV control in distribution networks with many distributed PVs. Further distributed voltage control analysis and results are presented in Appendix A.4.

\subsection{CONTROL DEVELOPMENT}

Figure 36 illustrates the data flows within the control system architecture. The system comprises a single element that implements the control logic. 


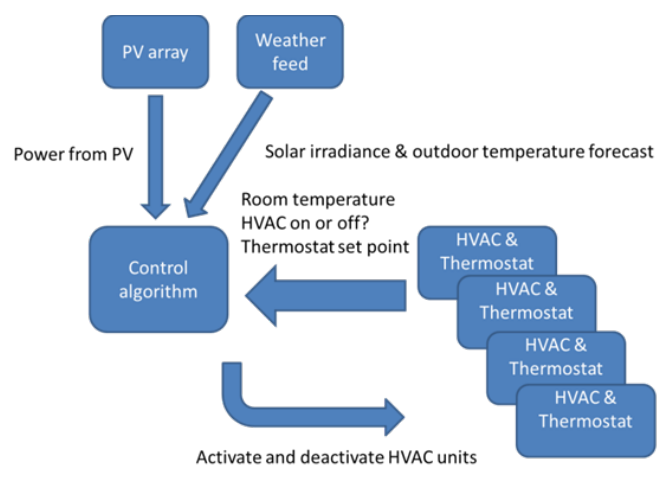

Figure 36. Control system architecture data flows.

This element receives data that relate output power from the PV unit, forecasts for solar irradiance and outdoor air temperature, zone temperatures relevant to the HVAC units being controlled, user-specified temperature set points in those zones, and the current condition (i.e., on or off) of the HVAC equipment. The data are supplied at a rate commensurate with the control period. Hence, if a 10 min control period is used, then these data streams will be sampled by the control at least once in every $10 \mathrm{~min}$ period. In practice, more frequent sampling might be necessary to smooth noise, estimate model parameters, or perform other data-processing tasks needed to support the primary function of the control. At the end of each control period, the control sends actuation commands to the HVAC units as is determined necessary by the control logic acting in response to its most recently received data.

The controller is a Python implementation that will ultimately be deployed on a cloud platform. The controller will then query data from the relevant endpoints and assign control decisions though a web application programming interface (API). The system architecture will be deployed as illustrated in Figure 37. Connections between subsystems will be wireless using site Wi-Fi unless otherwise required. Prospective cloud platforms include Amazon Web Services (AWS) EC2 web service. This widely used service has a reliable interface in the boto3 Python library and would be sufficient for the control deployment.

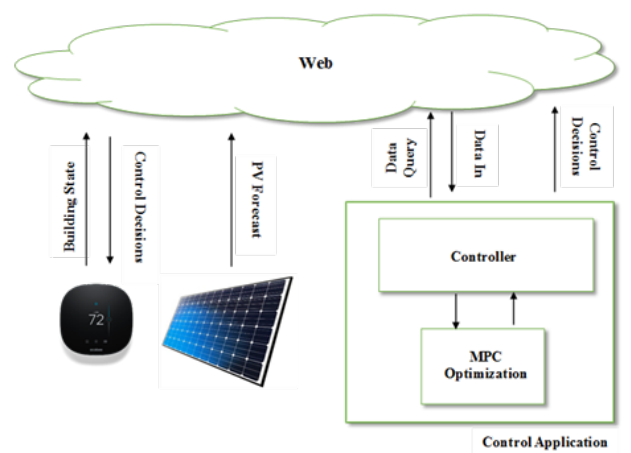

Figure 37. System architecture for deployment.

There are two endpoint classes for the system: controllable endpoints and data-gathering endpoints. Controllable endpoints relay state information and actuate the control decisions made by the controller. These include devices with controllable loads, such as HVAC units. Data-gathering endpoints will relay necessary data points for the control decisions, such as solar weather forecasts. Current project requirements include the use of commercial thermostats for the control of the HVAC loads and querying building state. Candidate hardware includes Nest and Ecobee thermostats. These products are currently being evaluated for project suitability. 
The overall system activity is illustrated in Figure 38. At the start of a control period, data will be queried and fed to the controller. The optimization will run and output the optimal control for the control period. At the start of the next control period, the process will be repeated. Data will be queried more frequently within control periods to ensure that sufficient data exist to compute the control decision and that decisions are made on as recent data as possible.

The primary components internal to the control algorithm box are shown in Figure 39. All data received from the outside world are stored for use in

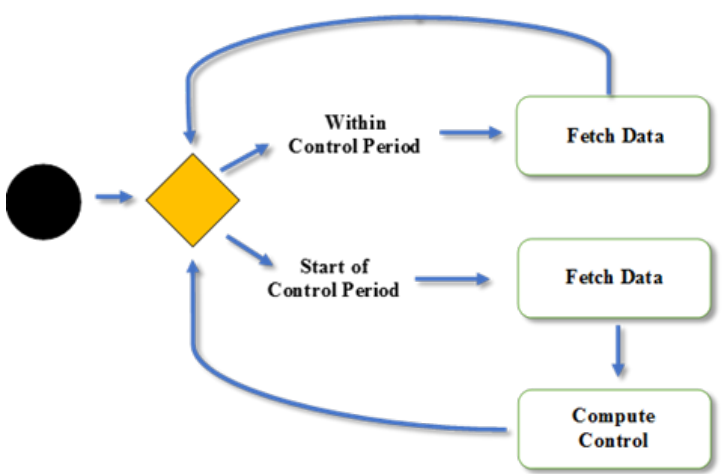

Figure 38. System activity. the online construction of models that will be used for forecasting. These forecasts are an essential part of the optimization procedure that selects the best choice of actuation commands. There are two forecasts essential for the control's operation. The first is a PV forecast, which is constructed from current and prior PV output data and the solar irradiance forecast. More details about the PV power forecast are presented in Appendix A.6. The second is a forecast of building temperature, which is constructed by using the same model used during system design but with parameters estimated from historical temperature, set point, and actuation data. More details about the building model parameters estimation are presented in Appendix A.5. The models used to generate these forecasts are integrated into a whole system model, and the optimization

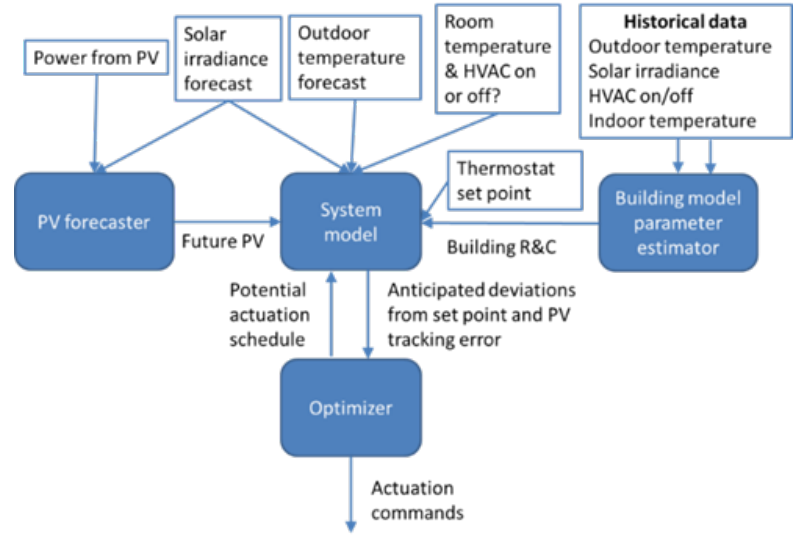

Figure 39. Primary logical components of the control algorithm. procedure explores the effects of specific actuation choices on the desirability of the forecasted system behavior. Desirability in this case is a weighted measure of the difference between power consumed by the collective HVAC units and power supplied by the PV array and deviation of actual room temperatures from end user set points.

In the field-testing demonstration (Section 2.6), the control algorithm resides on a single computer system. The thermostats reside physically adjacent to the relevant HVAC units and provide data to the control system computer via a wireless network or a cloud-based data repository. The latter is attractive due to the availability of commercial thermostat systems that provide the necessary telemetry data and actuation capabilities via a cloud-based interface. The PV array could provide data directly via a wired or wireless connection to a control computer that is physically near or via a cloud-based data repository.

The team developed a distribution feeder simulation platform in OpenDSS and designed an adaptive optimal control strategy in MATLAB (later in Python) to make HVAC loads track PV generation. There is a need to develop a co-simulation tool to show the line voltage and tap change differences after applying the adaptive control of HVAC loads. To address this, the team used the A Discrete Event System simulator (ADEVS) interface, which was previously developed at ORNL, to interact between MATLAB and OpenDSS, as shown in Figure 40. In contrast to existing techniques, ADEVS offers a comprehensive and accurate way of setting up the co-simulation environment. ADEVS offers friendly interfaces with MATLAB and OpenDSS so that users do not need to configure detailed settings. 
Specifically, control decisions obtained in MATLAB will be passed to actual load buses in OpenDSS, which enables the team to see real-time changes in-line voltage and tap change.

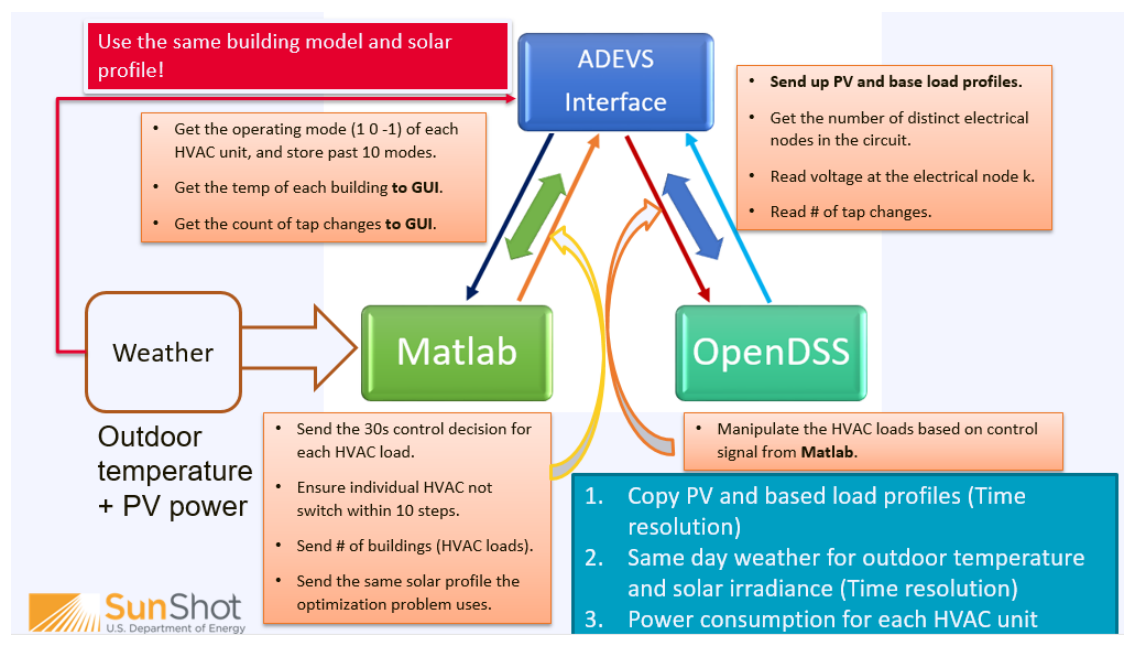

Figure 40. Co-simulation architecture.

Next, the team provided its initial investigation about the control system field testing and deployment. With the ultimate goal of implementing the controller inside a physical building, the following steps were followed to move toward this goal.

1. Implement the controller as deployable code.

2. Simulate and stress-test the system.

3. Install on appropriate hardware.

4. Install hardware in the building.

\subsubsection{Control System Testing}

The plan is for the control algorithm to be implemented as a Python application. Once the application is constructed, the code will be packaged in a virtual machine and tested by using the discrete event system simulator ADEVS, which allows production code to be simulated and tested by using a simulated building model and virtual HVAC equipment. This testing regimen allows the response of the application to stresses, such as a communications disruption and unexpected environmental changes, to be monitored. The results will be used for debugging and refinement. After a sufficient number of hours has been simulated to ensure application reliability, the code will be ready to be packaged on hardware.

\subsubsection{Control System Hardware}

The hardware that must be considered for the deployment will run the control algorithm and gather model inputs and the thermostats that will relay commands and data between the HVAC rooftop unit (RTUs) and the control. Currently, the control will be implemented on an Intel NUC single-board computer. The NUC will have sufficient processing power to solve the optimization problem required by the controller. The thermostats used to actuate the RTUs, and control temperature set points will be commercial-grade smart thermostats, such as the Nest or Ecobee. These thermostats have Wi-Fi access and a RESTful API, simplifying data gathering and potentially control actuation. 


\subsubsection{Simulation and Stress Testing}

The software code is packaged in a virtual machine and tested by using the discrete-event system simulator ADEVS. ADEVS allows production code to be simulated and tested by using a simulated building model and virtual HVAC equipment. This testing regimen allows the application responses to stresses, such as communication disruption and environmental changes, to be monitored. The results can be used for debugging and further algorithm refinement. After sufficient runs were simulated to ensure application reliability, the code was packaged on hardware.

\subsubsection{Field Evaluation}

The control application was installed and tested across several test sites. The first site was a gymnasium in Fountain City, Tennessee. The gymnasium has four 10 ton HVAC units and two 7.5 ton HVAC units that can be controlled. Solar power measurements will be taken from the solar power banks at the ORNL campus. Virtually linking these two components allows sufficient capacity to effectively test the PVfollowing goal of the control. Because the components exist on disparate physical locations, communications will be required to travel over the internet. This necessitates the use of cloud data storage, such as that provided by AWS.

Leveraging its simulation-based testing technology, the team aimed to couple building simulation models that simulated the physical environment and HVAC equipment via control software that incorporates the PV signal, mixed-integer optimization algorithm, and control logic. The goal was to demonstrate a testing platform that tests the operational software and its connectivity to the buildings as it will be deployed in the field. By doing so, the team was able to accumulate a significant numbers of testing hours for the operational software and test the scalability of the algorithm without a need for costly hardware deployments. The overall test case is illustrated in Figure 41.

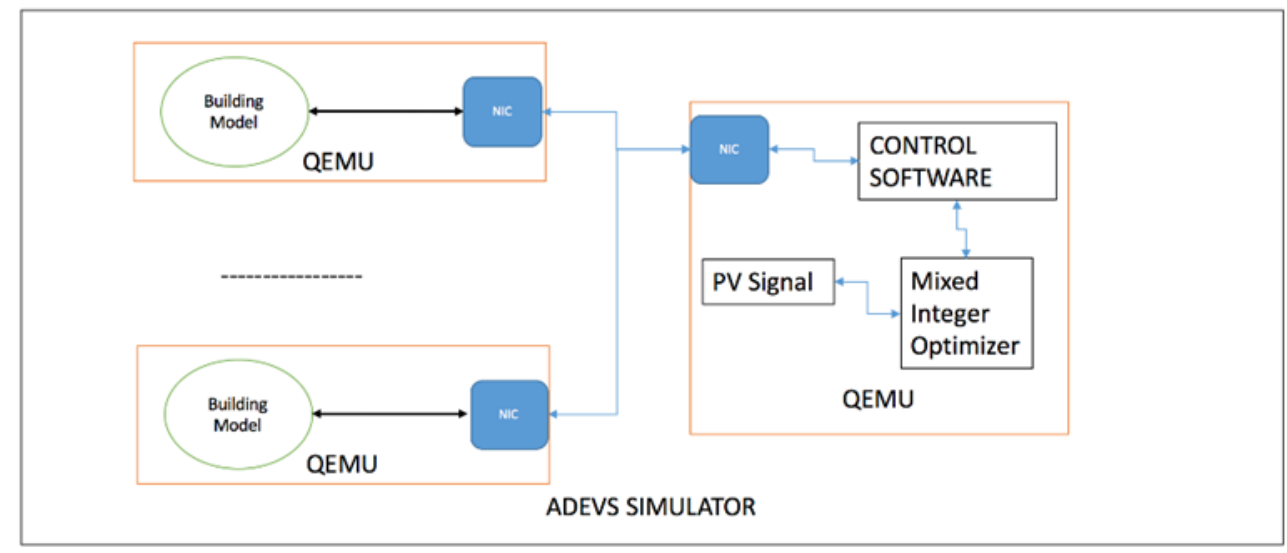

Figure 41. Simulation-based testing components for a fleet of TCLs.

\subsubsection{Adaptive Building Load Control}

Two control methods were developed in this project: a is model-based method and a model-free method. Only the model-based predictive control is presented in this report because it was tested in the field. The model-free control (MFC) was only tested in a simulation environment, and it is presented in Appendix A.7.

Traditional thermostat (dead band) on/off control is the basic/baseline control to be compared with the team's proposed adaptive optimal control. The team started by describing the HVAC model used in this 
work. The system model was proposed in Gwerder [59] and employed in Ma [60]. The following example involves a continuous-time linear time-invariant system based on the dynamics of the room-temperature, interior-wall surface temperature and the exterior-wall core temperature.

$$
\begin{aligned}
& \dot{t_{1}}=\frac{1}{C_{1}}\left[\left(K_{1}+K_{2}\right)\left(t_{2}-t_{1}\right)+K_{5}\left(t_{3}-t_{1}\right)+K_{3}\left(\delta_{1}-t_{1}\right)+u_{h}+u_{c}+\delta_{2}+\delta_{3}\right] \\
& \dot{t_{2}}=\frac{1}{C_{2}}\left[\left(K_{1}+K_{2}\right)\left(t_{2}-t_{1}\right)+\delta_{2}\right] \\
& \dot{t_{3}}=\frac{1}{C_{3}}\left[K_{5}\left(t_{1}-t_{3}\right)+K_{4}\left(\delta_{1}-t_{3}\right)\right]
\end{aligned}
$$

The parameters are defined in Table 3.

Table 3. HVAC model parameter definitions.

\begin{tabular}{|c|l|}
\hline Variables & \multicolumn{1}{|c|}{ Definition } \\
\hline$t_{1}$ & Room air temperature $\left({ }^{\circ} \mathrm{F}\right)$ \\
\hline$t_{2}$ & Interior-wall surface temperature $\left({ }^{\circ} \mathrm{F}\right)$ \\
\hline$t_{3}$ & Exterior-wall core temperature $\left({ }^{\circ} \mathrm{F}\right)$ \\
\hline$u_{h}$ & Heating power $(\geq 0)(\mathrm{kW})$ \\
\hline$u_{c}$ & Cooling power $(\leq 0)(\mathrm{kW})$ \\
\hline$\delta_{1}$ & Outside air temperature $\left({ }^{\circ} \mathrm{F}\right)$ \\
\hline$\delta_{2}$ & Solar radiation $(\mathrm{kW})$ \\
\hline$\delta_{3}$ & Internal heat sources $(\mathrm{kW})$ \\
\hline
\end{tabular}

All the other variables are treated as constants and estimated as described previously.

The system states are the room air temperature $t_{l}$, interior wall surface temperature $t_{2}$, and exterior wall core temperature $t_{3}$. The control signals $u_{h}$ and $u_{c}$ represent heating and cooling power, and they can be combined as one variable, $u=u_{\mathrm{h}}+u_{c}$, because heating and cooling are not simultaneous. Hence, the thermal model for each individual building is given in the state-space form as:

$$
\dot{X}=A X+B U+G V .
$$

This section provides only a summer cooling case. State-space matrices $A, B$, and $G$ can be obtained for any given building, and disturbance $V$ is recorded for that specific location and time.

This study focused on the summer cooling case. All the modeling and control technologies will also hold for the winter heating scenario. For summer cooling, the chosen set point was $26^{\circ} \mathrm{C}$, and $\mathrm{a} \pm 2^{\circ} \mathrm{C}$ comfort band was allowed. To make the total power consumption track the available power generation from solar panel, the controller had the freedom to fluctuate temperature inside this comfort band.

The team considered the problem in which indoor temperature $t_{l}$ is required to remain within certain bounds of a constant dead band in the presence of the disturbance vector $V$. The goal was to minimize the error $e$ to keep the temperature $t_{l}$ close to the desired value. The total control inputs were also required to follow given $\mathrm{PV}$ generation $P_{P V}$ signal, that is: 


$$
U_{\text {sum }}(t):=\sum_{j=1}^{N_{s}} u_{j}(t) \approx P_{P V}(t),
$$

where $N_{s}$ denotes total number of HVAC systems. Thus, the objective was to find for the building system, the $M$ time step control sequences $\left\{u_{0}, \cdots, u_{M-1}\right\}$, where $u_{i}:=u\left(t_{i}\right), i=0, \cdots, M ; M$ is an integer, $t_{i}=i \Delta T$, and $\Delta T$ is the sampling period; and corresponding state sequence $\left\{x_{0}, \cdots x_{M-1}\right\}$ and error sequence $\left\{e_{0}, \cdots, e_{M-1}\right\}$. The difference between total control signal and PV signal is denoted for each time step $k$ as:

$$
J_{u}(k)=\left(\sum_{j=1}^{N_{s}}\left(u_{j}(k)\right)-P_{P V}(k)\right)^{\prime} R\left(\sum_{j=1}^{N_{s}}\left(u_{j}(k)\right)-P_{P V}(k)\right) .
$$

The difference between total control signal and PV signal were considered together with state deviation:

$$
J_{1}=\sum_{k=1}^{N_{p}}\left\{\left[x(k)-x_{r e f}\right]^{\prime} Q\left[x(k)-x_{r e f}\right]+J_{u}(k)\right\},
$$

where $N_{p}$ represents the prediction horizon with $Q \geq 0, R>0$ being compatible dimensional matrices. Accordingly, $u_{j}(k)$ means control action taken for $j_{t h}$ building at $k_{t h}$ time interval.

Furthermore, the team intends to use as little power as we can to save energy. So, they consider the control inputs as a cost in the cost function:

$$
J_{2}=J_{1}+\sum_{k=1}^{N_{p}} U_{k}^{\prime} R_{2} U_{k}
$$

where $U_{k}$ aligns control actions for all the buildings at time step $k$ in a vector.

The team has both states and control inputs constraints in this problem. Because they have three states for each building, they set $x_{\min }=[24,0,0]^{\prime}, x_{\max }=[28,50,50]^{\prime}$.

Then for the control input, $u \in[0,1]$. Here, 0 means off, and 1 means $1 \mathrm{~kW}$ in the model. After defining constraints for states and inputs of each building, they can be easily aggregated for multiple buildings. Therefore:

$$
\begin{aligned}
& X_{\min }=\left[x_{\min } ; x_{\min } ; \cdots\right] ; X_{\max }=\left[x_{\max } ; x_{\max } ; \cdots\right] \\
& U_{\text {min }}=[0 ; 0 ; \cdots] ; U_{\max }=[1 ; 1 ; \cdots] .
\end{aligned}
$$

Additionally, the team has one more regulation requirement for $U_{\text {sum }}$ in Eq. (19).

Obviously, this is a linear quadratic programming problem that can be solved by any commercial solver.

The simulation results are for 45 buildings. In Figures 42-45, the left-hand side in each figure depicts the performance by using the proposed augmented optimal control technique. The right-hand side represents that of traditional control. Because the objective is to track the PV generation without deviating indoor 
temperature out of a bound, both variables are key to the design and are provided in Figures 42-45. Extremely good tracking results are obtained in Figure 44(a) during most of the simulation intervals. After comparing the sum of control signal with tracking reference (Figure 45), the team noticed that the tracking difference for the model predictive control (MPC) technique usually falls below $1 \mathrm{~kW}$. This indicates a huge improvement over the traditional optimal control. However, the tracking performance disappeared at the end of the simulation. Figure 43(a) shows that the problem is related to control input saturation. One potential reason for this issue is that the number of buildings is incompatible with the given PV generation. In other words, it becomes infeasible to use some number of buildings to track a given solar PV profile. Hence, a scenario was set up in which solar PV tracking is the top priority. A relaxed optimization problem is formed by removing the temperature constraints temporarily when optimization problems run into infeasible issues. The tracking performance is improved, as shown in Figure 44.

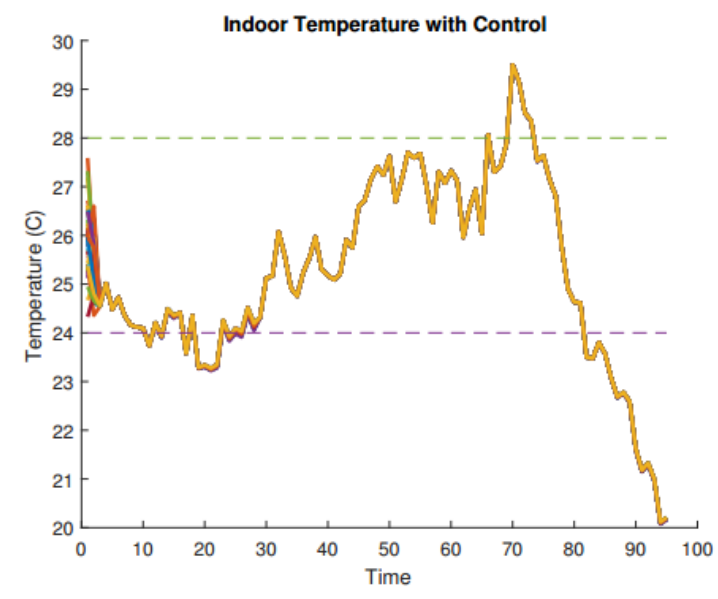

(a)

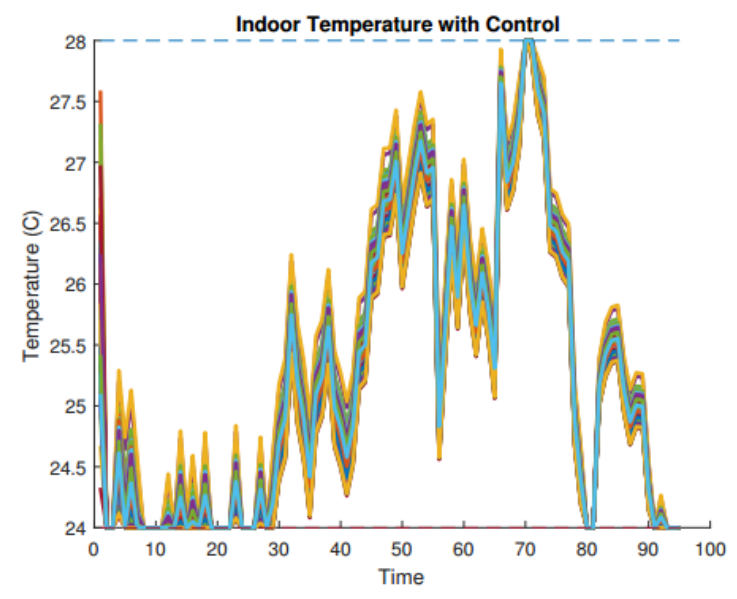

(b)

Figure 42. Comparison of indoor temperatures for 45 buildings by using (a) MPC and (b) traditional control.

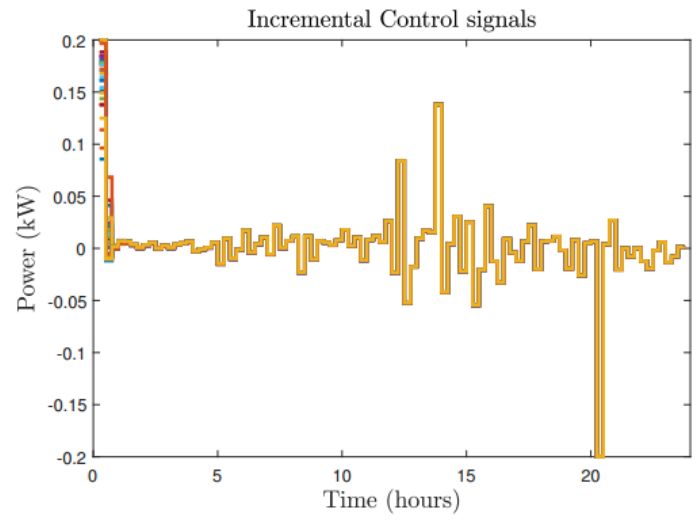

(a)

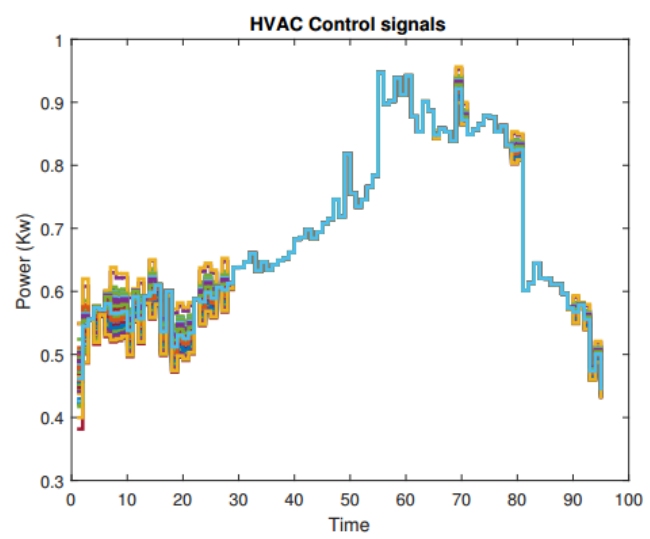

(b)

Figure 43. Comparison of control signals for (a) incremental control signal (fourth state) and (b) by using traditional control. 


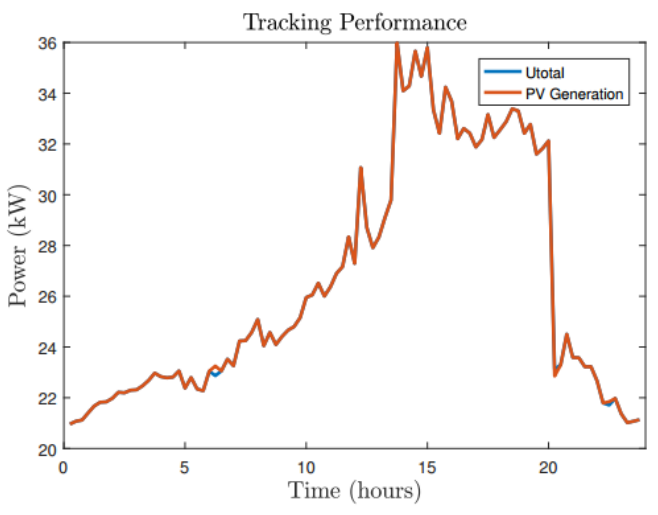

(a)

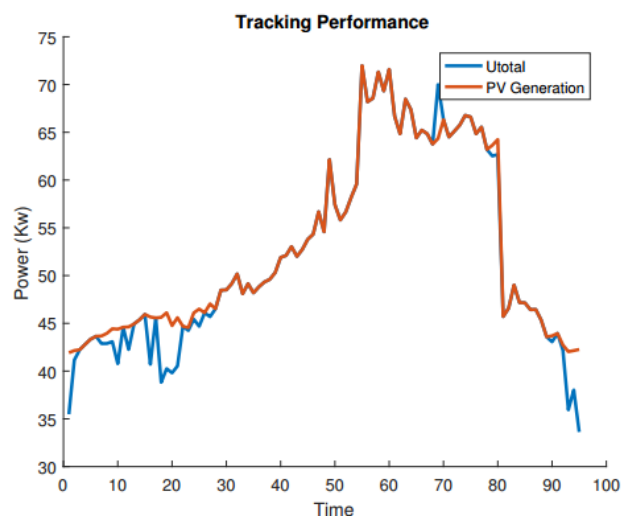

(b)

Figure 44. Comparison of tracking performance by using (a) MPC and (b) traditional control.

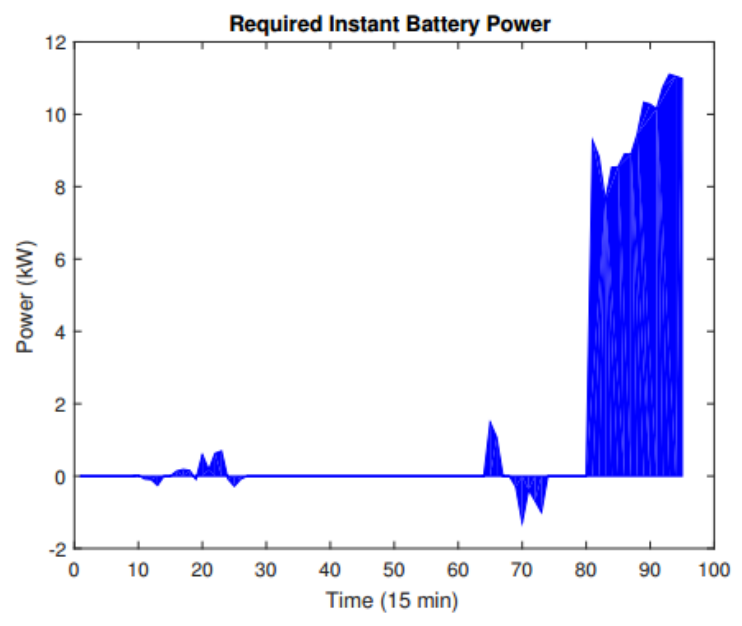

(a)

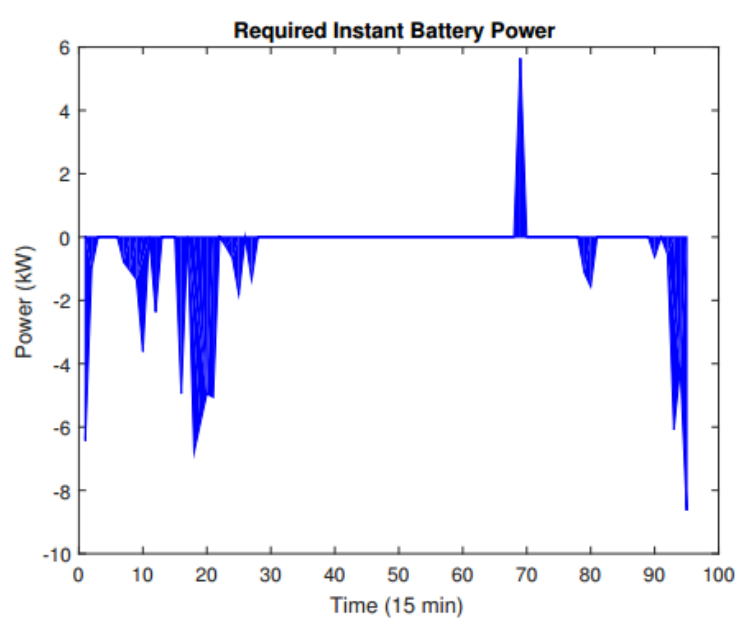

(b)

Figure 45. Comparison of battery usage by using (a) MPC and (b) traditional control.

The team also considered a generic mathematical model of a WH whose temperature $\left(t_{4}\right)$ at time instant $k$ is a function of the water temperature at the previous time instant $k-1$, the average hot water use, and the $\mathrm{ON} / \mathrm{OFF}$ state of the $\mathrm{WH}$ at time instant $k$, described as:

$$
t_{4}(k)=t_{4}(k-1)+\tau\left[\alpha_{W H} u_{W H}(k)-\beta_{W H} W(k)-\gamma_{W H}\right],
$$

where:

$\alpha_{W H}$ is a warming effect of an ON state of WH on water temperature (1.44),

$\beta_{W H}$ is a cooling effect of an OFF state of WH on water temperature (0.068),

$\gamma_{W H}$ is a cooling effect of activity level on temperature of WH (0.05), and

$W(k)$ is an average hourly hot water usage at time $k$.

Similarly, this model can be written into state-space form, as in Eq. (18). Hence, multiple variables will be added into the formulation. For example, $t_{4}$ was added into states vector $X, u_{W H}$ was added into control vector $U$, and $W$ was added into disturbance vector $V$. 
The effect of the ON state on WH water temperature increase is represented by $\alpha$, and the heat-loss effect of the OFF state of the $\mathrm{WH}$ is modeled by $\beta$. The latter is to address the thermal leakage due to the difference between the water temperature in a WH and the outside temperature. The effect of the activity level on the WH water temperature is modeled by using $\gamma$ so that as the household activity level increases, there is more demand for hot water. Customer comfort levels were considered, and the WH temperature was assumed to vary from 48 to $58^{\circ} \mathrm{C}$. Here, the control signal $u_{W H}$ is considered a discrete $\mathrm{ON} / \mathrm{OFF}$ variable.

The objective function of the MPC is formulated as follows:

$$
J_{u}(k)=\left(\sum_{j=1}^{N_{s}}\left(u_{j}^{H V A C}(t)+u_{j}^{W H}(t)\right)-P_{P V}(k)\right)^{\prime} R\left(\sum_{j=1}^{N_{s}}\left(u_{j}^{H V A C}(t)+u_{j}^{W H}(t)\right)-P_{P V}(k)\right),
$$

subject to HVAC dynamics in Eq. (17), WH dynamics in Eq. (24), and $x_{j}^{\min }(k)<x_{j}(k)<x_{j}^{\max }(k)$.

The basic idea is to track the PV profile by using HVAC and WH loads without violating temperature constraints.

In this report, the team first added the quantified duty cycle and energy-saving comparison for an aggregated $100 \mathrm{HVAC}$ units. Then, they showed results with a WH by using a discrete ON/OFF control strategy. Although only the summer cooling case was shown, all the modeling and control technologies will also apply to the winter heating scenario. For summer cooling, the chosen set point was $23^{\circ} \mathrm{C}$, and a $\pm 0.5^{\circ} \mathrm{C}$ comfort band was allowed. To make the total power consumption track the available power generation from a solar panel, the controller can fluctuate temperature inside this comfort band. A numerical example is provided to compare the tracking performances between basic control and adaptive optimal control. In the latter control strategy, the team considered a central coordinator that collects total PV generation and allocates energy to a group of HVACs to minimize the difference between total consumption and PV generation. In both simulations, 100 buildings were used, each of which is equipped with the identical 1R1C HVAC model. The simulation runs for $24 \mathrm{~h}$ with 10 min time steps. PV generation and weather profiles were both picked for the same day from a local station. All numerical simulations were coded in MATLAB (later in Python) and were solved by using Gurobi [61] (later using an open-source optimizer) through the YALMIP interface [62].

The $\Delta T_{h}$ and $\Delta T_{\mathrm{c}}$ terms were chosen to be $0.5^{\circ} \mathrm{C}$ in the basic control, and the reference temperature was set to be $23^{\circ} \mathrm{C}$, although the comfort band for adaptive optimal control is $\left[22.5^{\circ} \mathrm{C}, 23.5^{\circ} \mathrm{C}\right]$. Figures 46 and 47 compare the duty cycle and power consumption. The team randomly pick Building 18 and noticed that the duty cycle and energy consumption were reduced by more than $15 \%$. Then, to quantify the overall 100 buildings, the team plotted the average values across all HVAC units, as shown in Figure 47.

Numerical results indicate that the averaged duty cycle and energy consumption were reduced by $22 \%$ and $26 \%$, respectively. Therefore, the team claims that the optimal coordination that uses MPC will not affect the life cycle or efficiency of the HVAC system. 


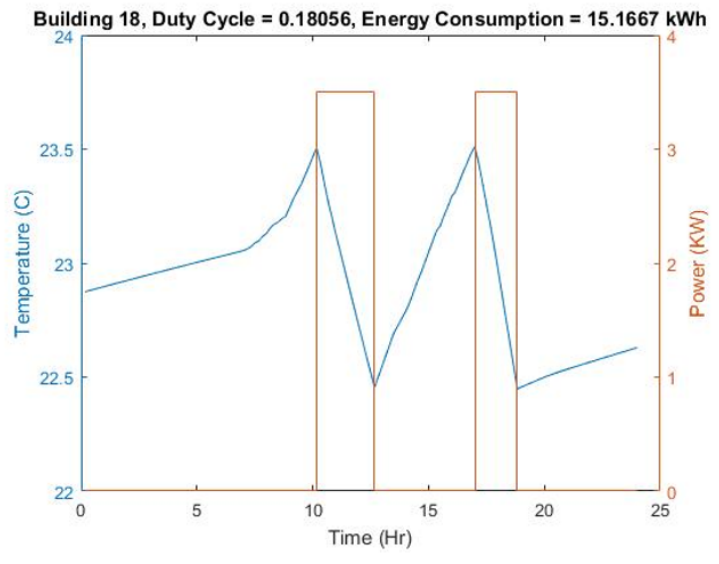

(a)

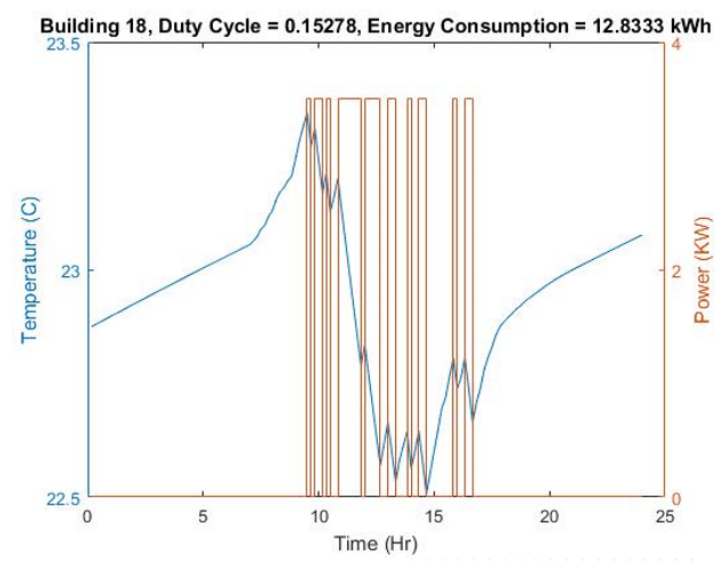

(b)

Figure 46. Duty cycle and energy consumption comparison for Building 18 for (a) basic control and (b) MPC.

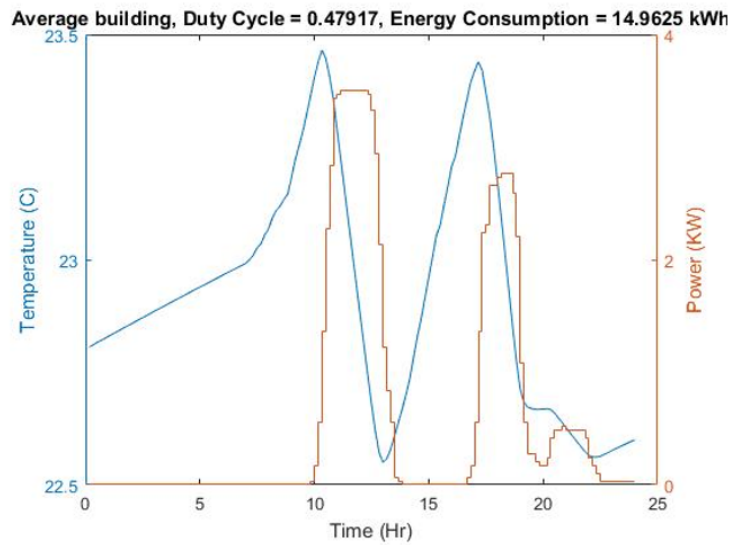

(a)

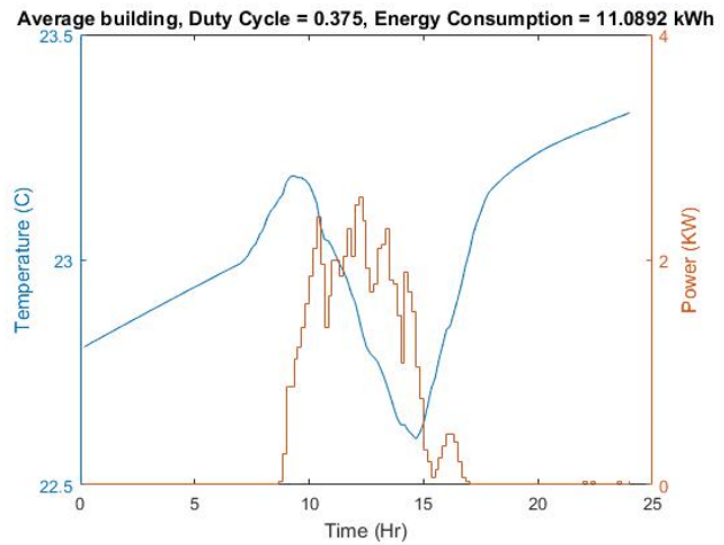

(b)

Figure 47. Duty cycle and energy consumption comparison for average building for (a) basic control and (b) MPC.

In the second part of this numerical study, the team replaced half the amount of the HVAC systems by WHs. A typical water usage profile is plotted in Figure 48. Then, the optimization problem was solved with results presented in Figures 49-53. Figure 49 shows that 50 HVAC and 50 WH loads are well coordinated to track the PV generation by using the proposed adaptive optimal control. Figures 50 and 51 show that the hot water and indoor temperatures are both within the desired comfort band. Additionally, the corresponding control decisions shown in Figures 50 and 51 are discrete ON/OFF signals. Figures 52 and 53 provide a detailed view of the temperature and control signals for Building 8 . The water temperature kept dropping before time step 50 because the WH was not turned on and there was water use. Moreover, Figure 52 shows more power consumption than was expected after time step 100 because the team increased water use during the night. Then, more WH units must turn on to maintain the water temperature at the lower bound, as shown in Figure 50. Therefore, the obtained results are optimal for HVAC and WH by cross-validating the figures. Now, to ensure that the controller will not put TCL devices under frequent ON/OFF switching conditions, which could reduce their lifetimes, the team included a third cost function in the optimization formulation that adds a penalty to HVAC state switching and investigated the change in HVAC on/off switching based on different PV penetration 
levels. The simulation carries the same simulation setup, which involves 100 buildings. The switching count adds one each time there is a state switch from ON to OFF and from OFF to ON.

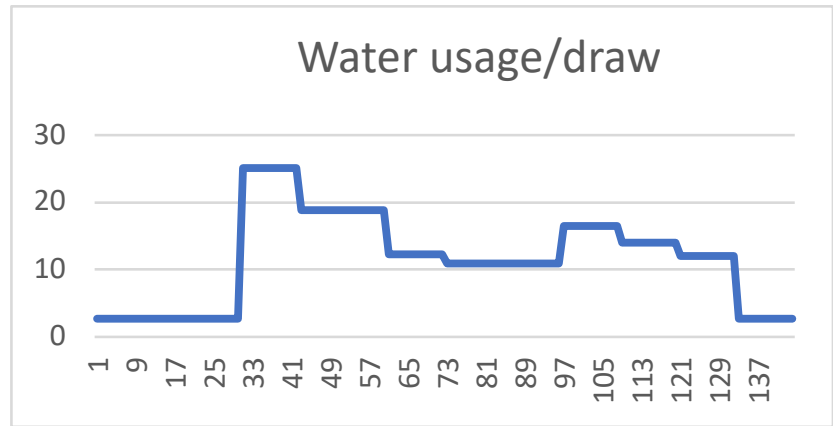

Figure 48. Water use profile.

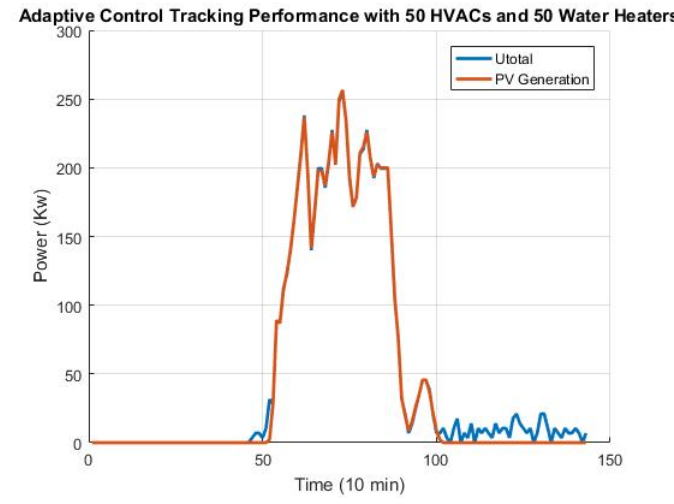

Figure 49. Tracking performance with 50 HVACs and 50 WHs.
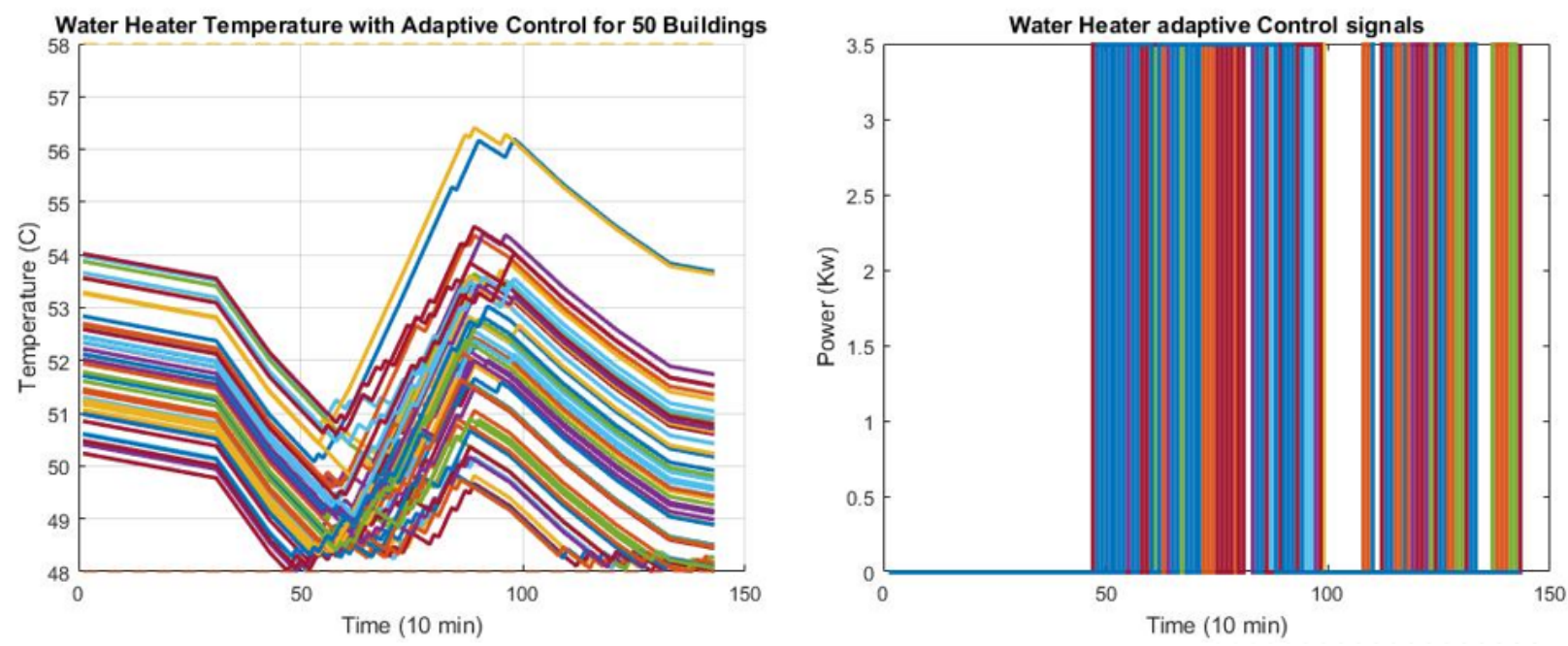

Figure 50. Temperature and ON/OFF control signal for WHs. 

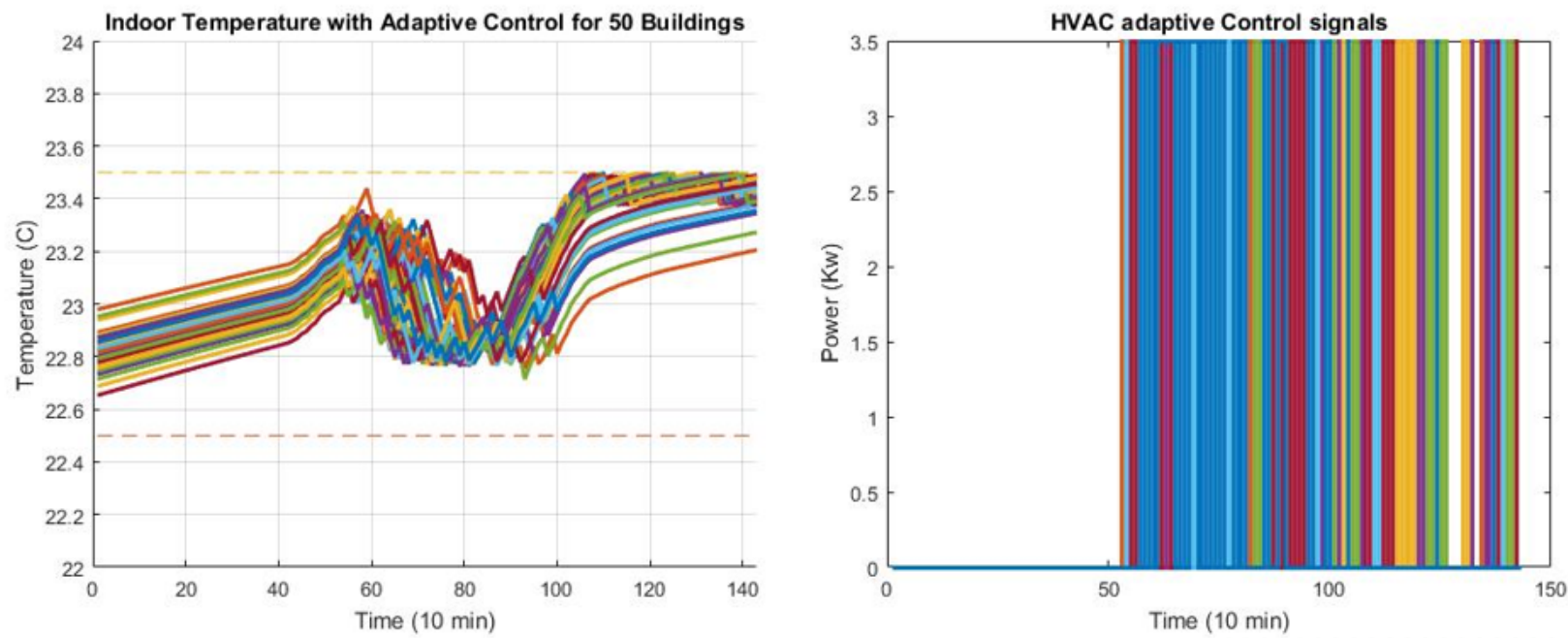

Figure 51. Temperature and ON/OFF control signal for HVACs.
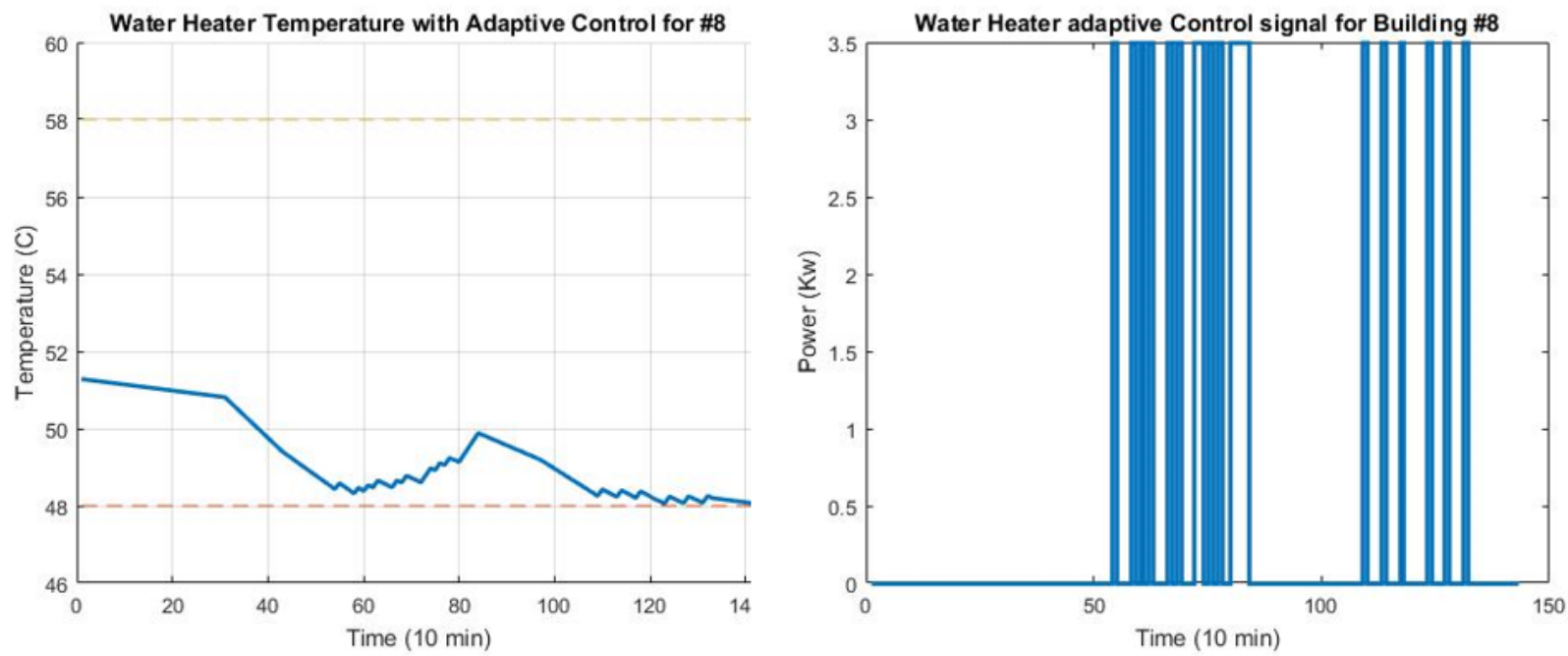

Figure 52. Temperature and ON/OFF control signal for WH in Building 8. 

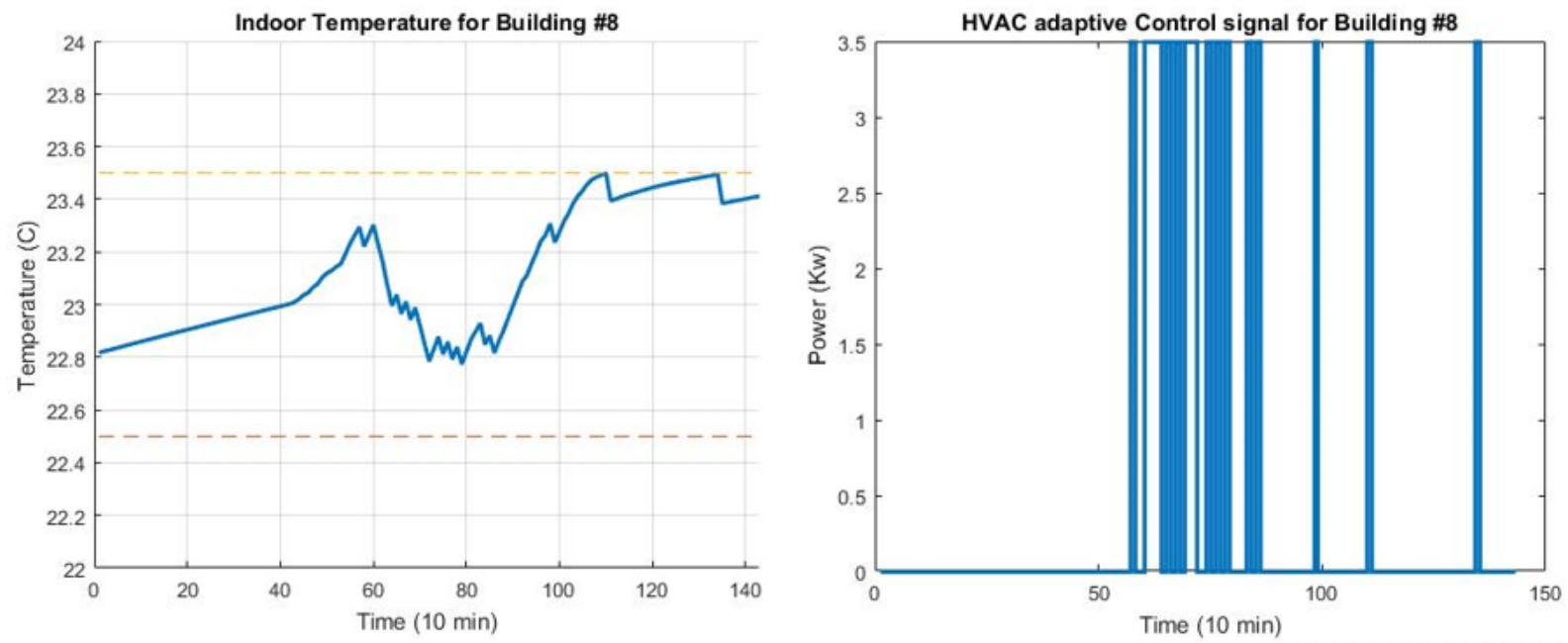

Figure 53. Temperature and ON/OFF control signal for HVAC in Building 8.

Figure 54 illustrates the daily total switching counts for each building. The team observed that HVAC switching has nearly doubled due to the developed adaptive control strategy compared with the basic thermostat-based control. However, this double switching behavior is still considered to be a reasonable operating condition for HVAC systems. Moreover, Figure 55 shows that the required HVAC switching can be reduced by increasing the number of units subscribed to provide service. On the other hand, with a certain number of PV penetration, we might increase the number of assigned HVAC units to avoid the frequent switching of each HVAC system.

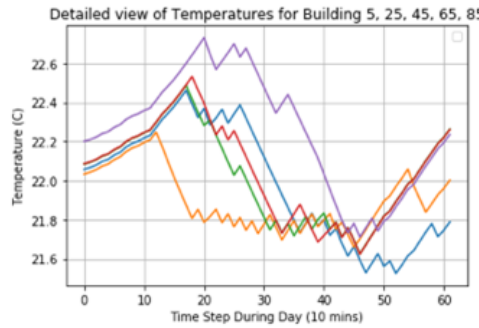

(a)

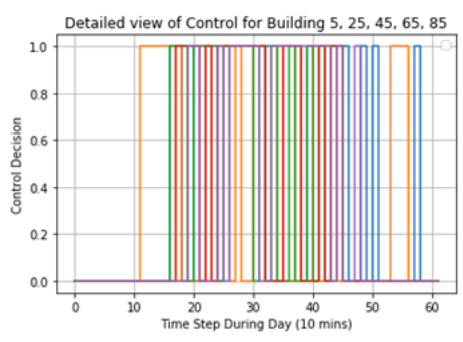

(b)

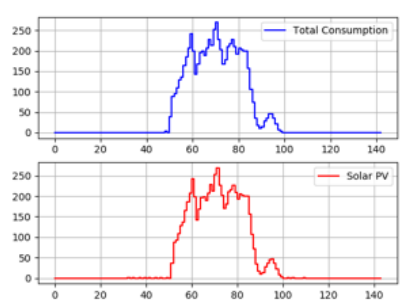

(c)

Figure 54. Simulation results for 100 ON/OFF HVAC systems using PuLP. (a) Indoor temperature $\left({ }^{\circ} \mathrm{C}\right)$, (b) continuous control signals $(\mathrm{kW})$, and (c) tracking performance $(\mathrm{kW})$. 


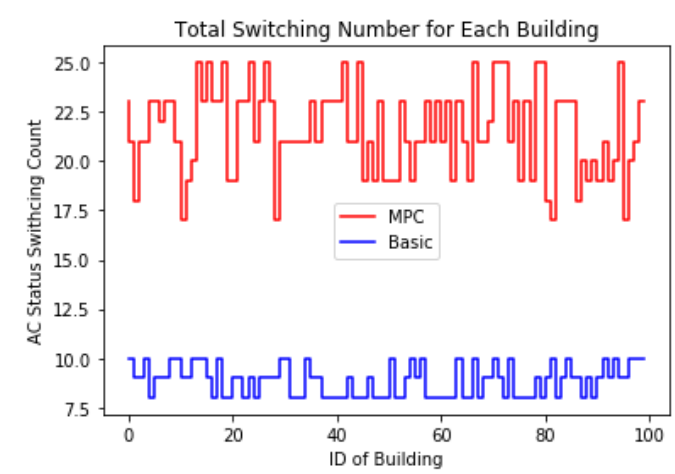

(a)

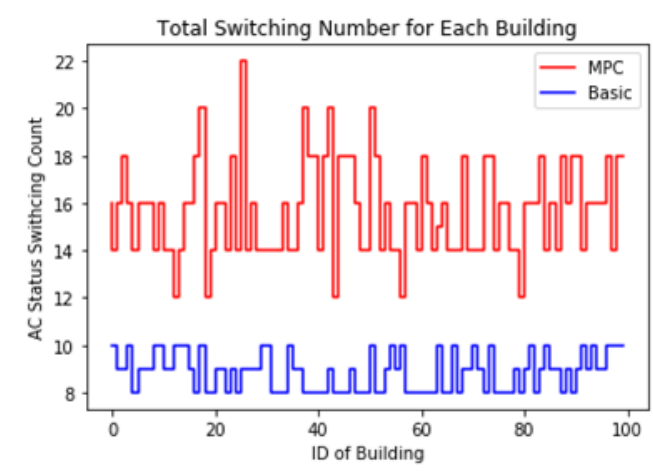

(b)

Figure 55. Daily HVAC switching count with (a) $50 \%$ and (b) $30 \%$ PV penetration.

In the presented simulation results, it was assumed that perfect forecasting can be achieved for solar PV generation. However, in reality, the predicted PV output power usually deviates from true values due to uncertainty in weather and cloud movement. Therefore, it is critical to evaluate and quantify the effect of PV prediction error on the tracking performance. Thus, the team considered two of the most popular types of prediction errors: the persistent model and the random noisy model. In the persistent model, the last PV measurement will stay unchanged to represent future solar PV generation values during the whole prediction horizon. Intuitively, the team assumed that PV generation will remain the same for a certain time interval that changes from 20 to $60 \mathrm{~min}$ in $10 \mathrm{~min}$ increments. The tracking performance for different persistence intervals is illustrated in Figure 56 with the corresponding tracking errors.

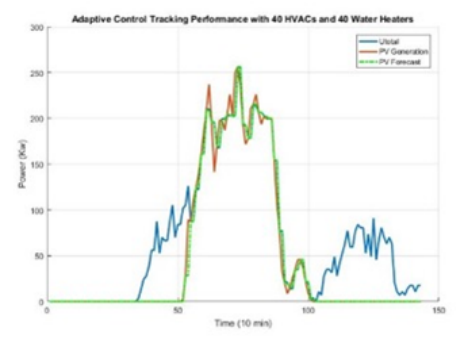

(a)

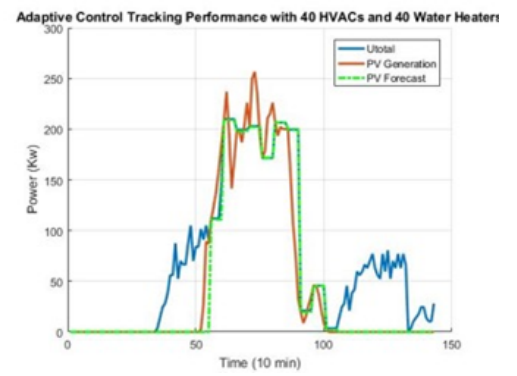

(d)

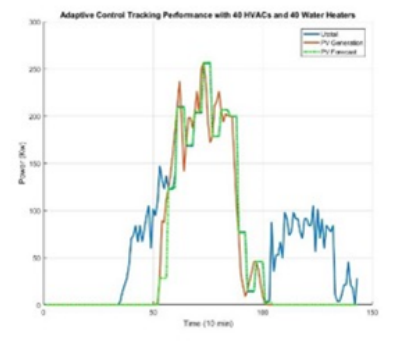

(b)

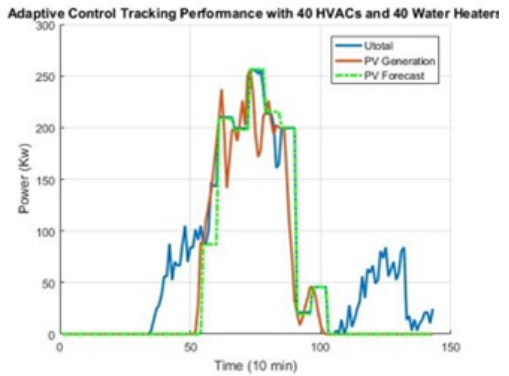

(e)

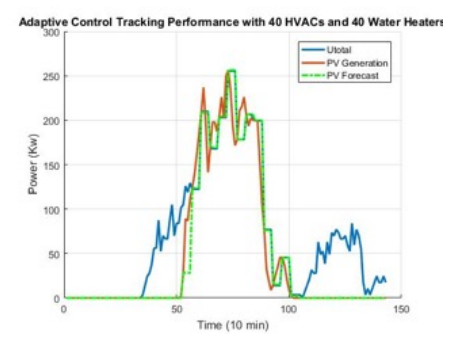

(c)

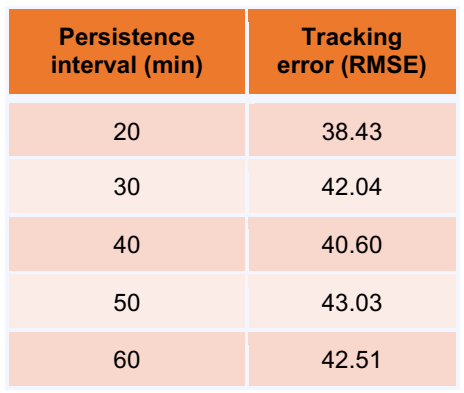

(f)

Figure 56. Tracking performance for PV forecasting error by (a) 20, (b) 30, (c) 40, (d) 50, and (e) 60 min persistence intervals and (f) their corresponding tracking errors. 
Subsequently, the team switched to the second scenario in which random noise is added to the original accurate solar PV generation. Similarly, the team investigated the tracking errors that correspond to different levels of noise described by the ratio between magnitudes of added random noise to the solar PV generation. Specifically, the noise level was increase from 10 to $50 \%$. The corresponding tracking performance is illustrated in Figure 57 with the corresponding tracking errors. As expected, the tracking performance becomes worse with enlarged noise added to the model. However, the degradation in percentage is still acceptable, even with $50 \%$ random noise. This provides a promising outcome because the tracking performance after real deployment will not suffer from severe degradation considering large solar forecasting errors.

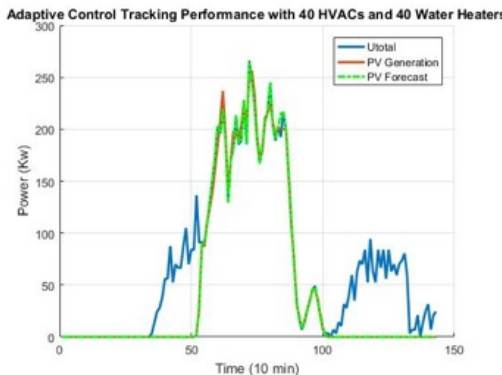

(a)

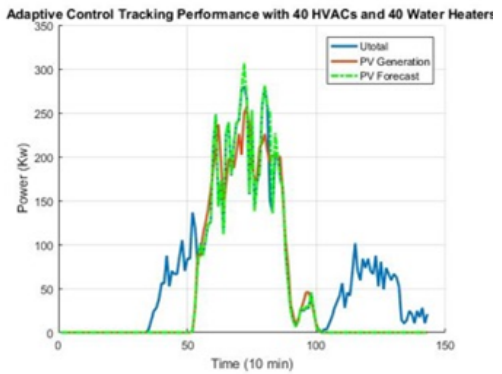

(d)

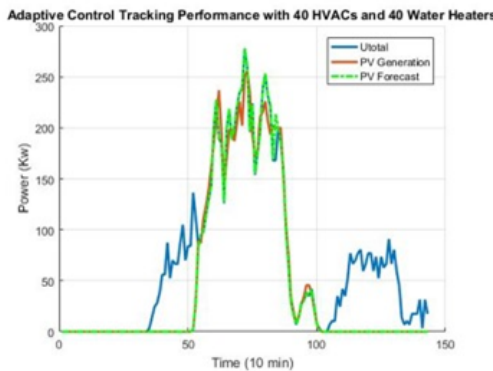

(b)

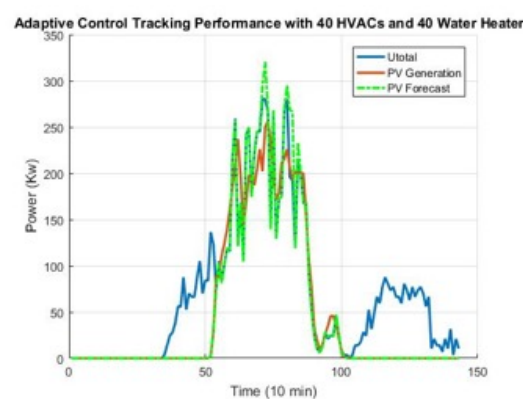

(e)

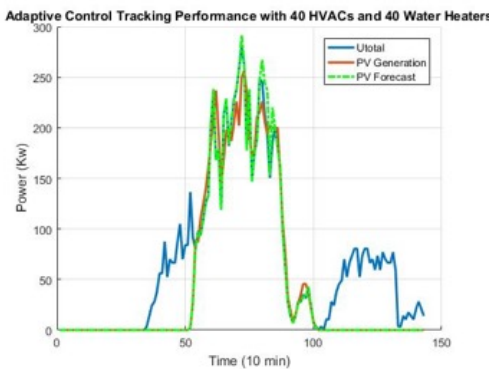

(c)

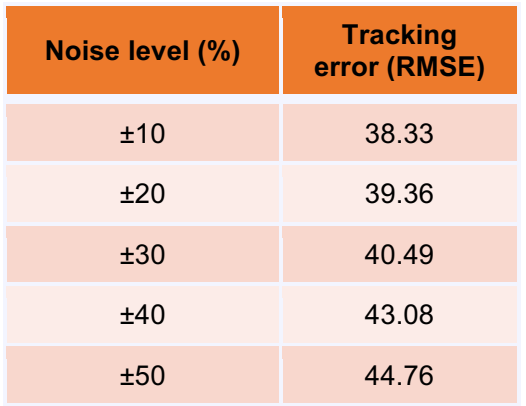

(f)

Figure 57. Tracking performance for PV forecasting error represented by (a) 10, (b) 20, (c) 30, (d) 40, and (e) $50 \%$ noise level and (f) their corresponding tracking errors.

\subsection{CONTROL DEPLOYMENT}

The dynamic models were used to develop a model-based control design environment and test bed. The goal was to create a formal statement of the control problem that reflects the objective: to use load control as a reliable, deployable technique to offset the impact of PV output variations on distribution voltage regulation. An iterative model-based control design process, as shown in Figure 58, coupled with test facilities that can be programmed with various disturbances provided a unique opportunity to explore control challenges in an agile software development environment. 


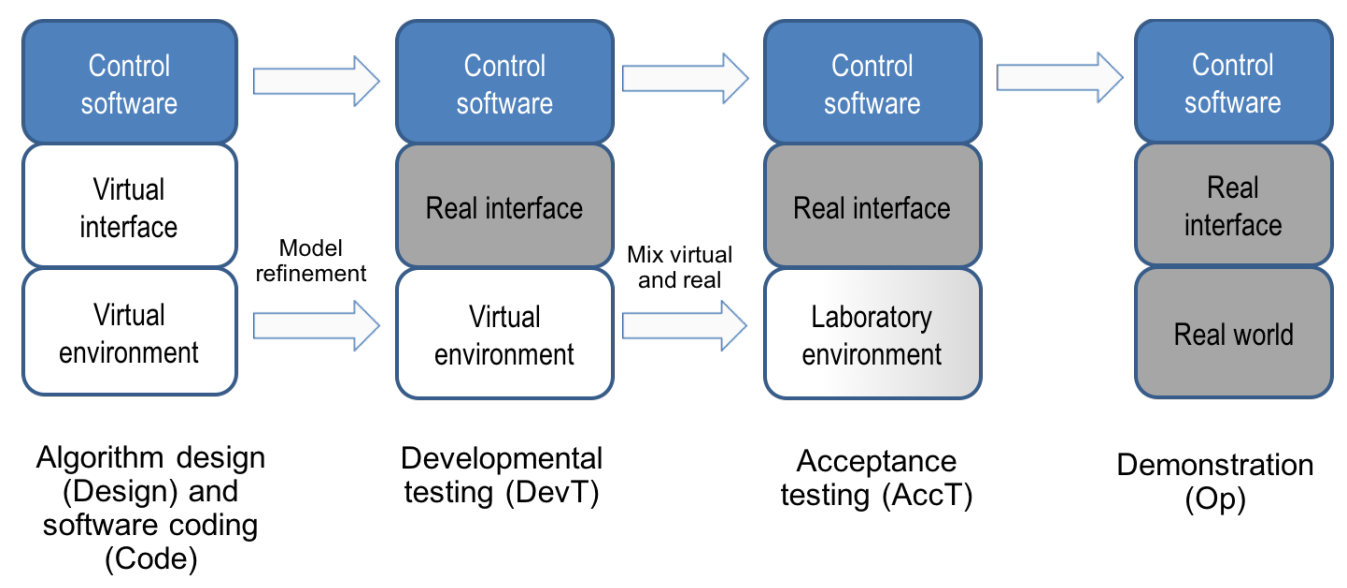

Figure 58. Incremental approach to control development.

First, the team transferred the theoretical control research to practical implementations of deployable code. For implementation onto hardware, the MATLAB research code was converted to Python. The chosen optimization interface was PICOS [63], a user-friendly interface to several conic and integer programming solvers, very similar to YALMIP or CVX under MATLAB. A free optimization solver called ZIBOPT [64] was used to solve the continuous control problem, and the enhanced Gurobi solver was used to solve the mixed integer programming to obtain a discrete ON/OFF control signal.

The team developed a deployment-ready Python-based solution for performing the optimal coordination of TCLs. Their previous design environment included MATLAB for exploring several dimensions of controller performance. This control technology was implemented by using open-source software and solvers to perform near-real-time optimization for tracking solar PV output by using building loads and dispatch of control set points. Specifically, PuLP [65] was chosen as the Python optimization interface, and COIN-OR [66] was chosen as the free optimization solver. PuLP is a linear programming (LP) optimization modeler written in Python that can generate LP files and call GLPK, COIN CLP/CBC, CPLEX, and GUROBI to solve linear problems. COIN-OR has more than 50 application projects, including tools for linear programming (e.g., COIN-OR CLP), nonlinear programming (e.g., IPOPT), integer programming (e.g., $\mathrm{CBC}$, Bcp, and COIN-OR SYMPHONY), and algebraic modeling languages (e.g., Coopr). Based on this new Python framework, the team reformulated its MPC problem and regenerated the results to calibrate the implementation. The new optimization solver is tuned for performance and is demonstrated to be more computationally efficient than the MATLAB-based implementation. On one typical i7 desktop, for 100 buildings, the team was able to compute the optimal scheduling for each time step in less than $0.25 \mathrm{~s}$. This demonstrated the feasibility of resolving the optimization for online implementation. Before using this approach, the team went through all the existing options for solving mixed-integer quadratic programming optimization in Python to ensure that the open-source solution was compatible with the state-of-the-art optimization solution. The team also compared the new approach with the PICOS conic optimization solver interface [63], which calls the most popular commercial solvers, including CPLEX, GORUBI, and MOSEK.

The new optimization solver is much more efficient than the one developed in the MATLAB platform. On one typical i7 desktop, for $100 \mathrm{HVAC}$ systems, the team was able to compute the optimal scheduling for each time step within less than $0.25 \mathrm{~s}$. This capability has completely resolved the optimization bottleneck for the online implementation. It has also enabled a fully open-source solution for this adaptive PV tracking problem and enabled a wide implementation of this control optimization algorithm on any platform. 
MPC deployment necessitates the development of four key components: (1) temperature and state measurements, (2) system identification, (3) outdoor temperature and solar forecasting, and (4) MPC control.

All controller components were created by using Python, and they use open-source software packages. Temperature and state measurements were gathered by querying customer-owned smart thermostats. Currently, the controller supports Ecobee thermostats and gathers the needed measurements through the Ecobee RESTful API. System identification was performed by a software module by using a least-squares regression model. Outdoor temperature and solar forecasting were performed by a module that gathers zip code-level data from an external source, such as Solar Underground. The module then uses autoregression (AR) based on previously observed data. The MPC control was calculated by using the COIN-OR opensource solver with the PuLP interface. These modules are housed within a controller, which can be deployed either virtually or through a web virtual server, such as the Amazon E2. Figure 59 shows the system architecture. Figure 60 demonstrates the system architecture within the controller. The subsequent sections describe each module in more detail. The final software package comprises 2,235 lines of Python code, not including any additional code required to deploy the software into different environments. For distribution, this code will be uploaded to a public code repository, such as GitHub or GitLab. More details on controller deployment are presented in Appendix A.8.

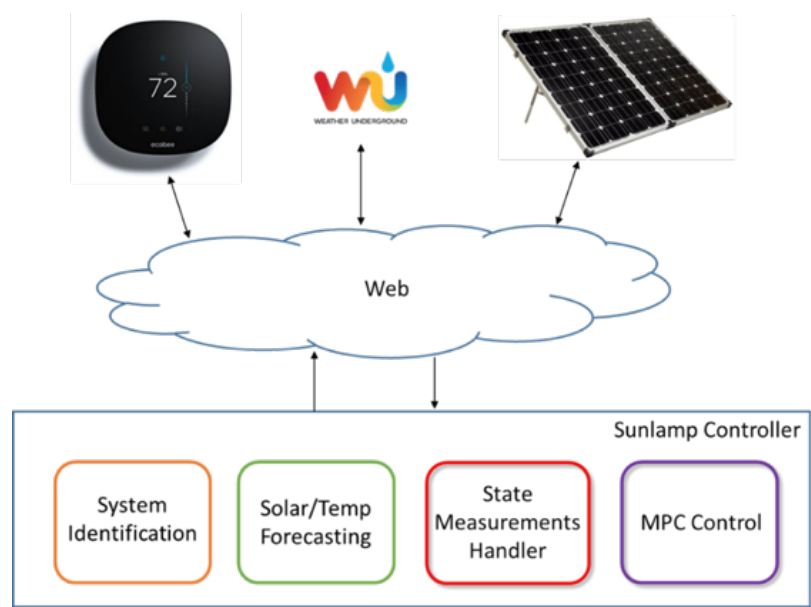

Figure 59. Controller infrastructure with external data sources.

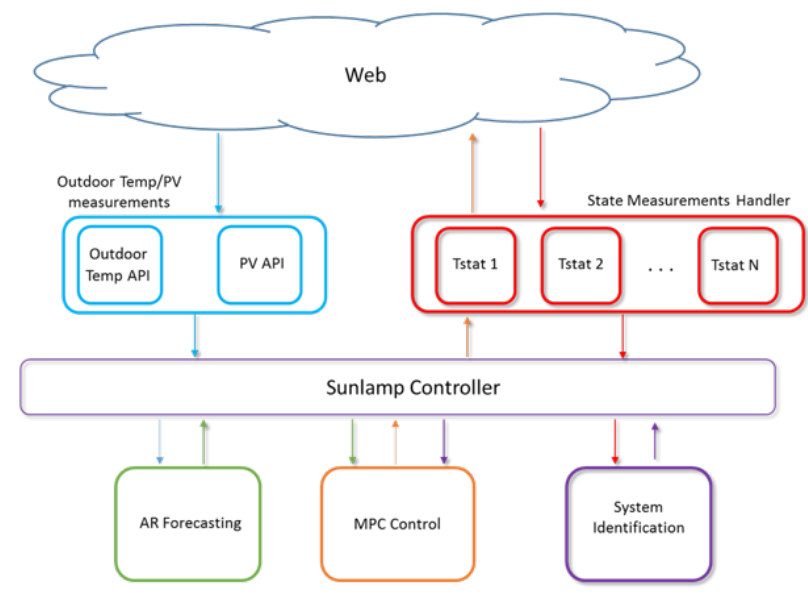

Figure 60. Internal controller architecture.

\subsubsection{State Measurement}

The state measurements that the MPC controller requires were taken from smart thermostats that exist within the resources providing the load flexibility. Current implementations support the Ecobee smart thermostat exclusively. Ecobee provides a RESTful API, which can be used to query the thermostat states and actuate the MPC controls. Ecobee also provides security for accessing the thermostats in the form of tokens that must be acquired before accessing the thermostat API. These tokens expire every $24 \mathrm{~h}$ but can be automatically reacquired by authorized users for up to 1 year; however, potential issues could remain in using the APIs that the smart thermostat vendors provide. Specifically, the flow of information is ratelimited and dependent on a third-party server. Utilities are generally developing middleware to maintain reliability issues in such settings. For example, Southern Company is abstracting the vendor API by providing a middleware API to access the devices while managing third-party vendors. 


\subsubsection{System Identification}

Because of the relatively slow dynamics of the individual buildings, a least-squares regression was determined to provide the best trade-off on accuracy and computational complexity for the short-term forecasting that MPC requires. Additionally, each HVAC zone was taken as an individual system to be identified. Figure 61 compares the identified state-space matrices and the true state-spaces used in the testing model. Despite inaccuracy in the identification of the $B$ matrix, the effect on the results was minimal.

\subsubsection{Forecasting}
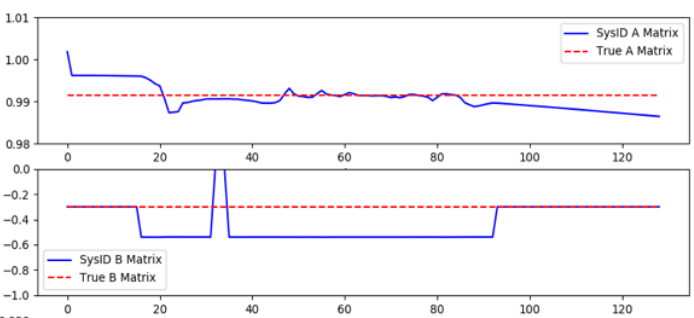

020 - SysiD G Matix

0.015 - - SyslD G Matrix

0.010 -

0.005 -

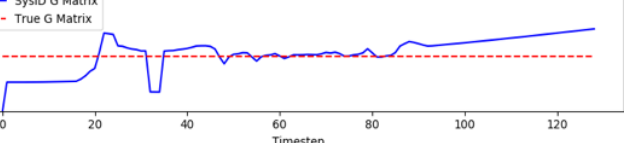

Figure 61. State-space model estimation via least-square system identification vs. true state-space model.

$\mathrm{AR}$ is a time series model that uses observations from previous time steps as input to a regression equation to predict the value at the next time step. In the deployment, this is taken care of by the "statsmodel" Python library. To reduce inaccuracy within the forecast and due to the relatively slow dynamics, for each control period, the disturbance is forecasted one time step (10 $\mathrm{min})$ into the future, and that forecast is used for the six time steps required to be forecasted by the MPC. Figure 62 shows the effect of forecasting PV one time step into the future throughout the simulation run.

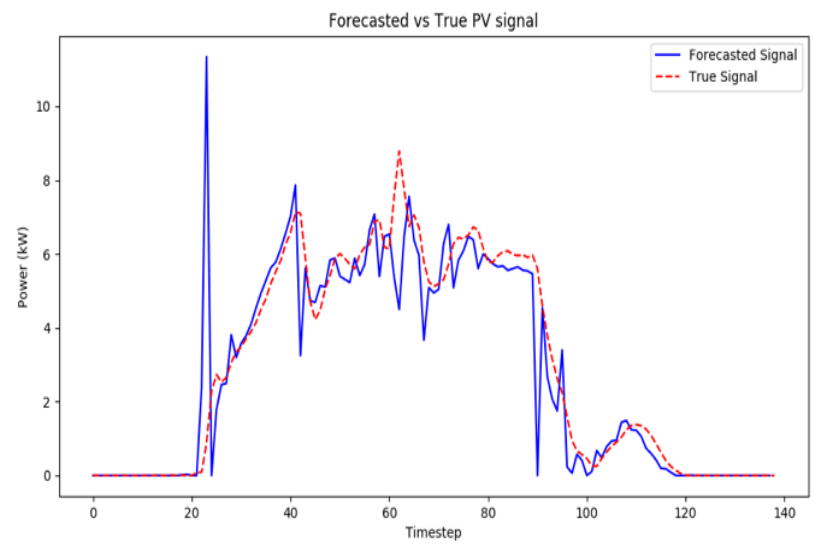

Figure 62. Auto regression PV forecast vs. true signal.

\subsubsection{MPC Controller}

The MPC is packaged as a separate module within the controller. It is called every control period (10 $\mathrm{min})$ and relies on projecting building state and outdoor disturbance six time steps $(1 \mathrm{~h})$ into the future. The solver used is the COIN-OR open-source solver.

\subsubsection{Controller Workflow}

Figure 63 illustrates the workflow of the controller, starting from the initial query of the thermostats. Thermostats that have dropped off the network are kept in a separate list, and communication is attempted at regular intervals. Furthermore, all historical measurements are logged in a separate MySQL database. 


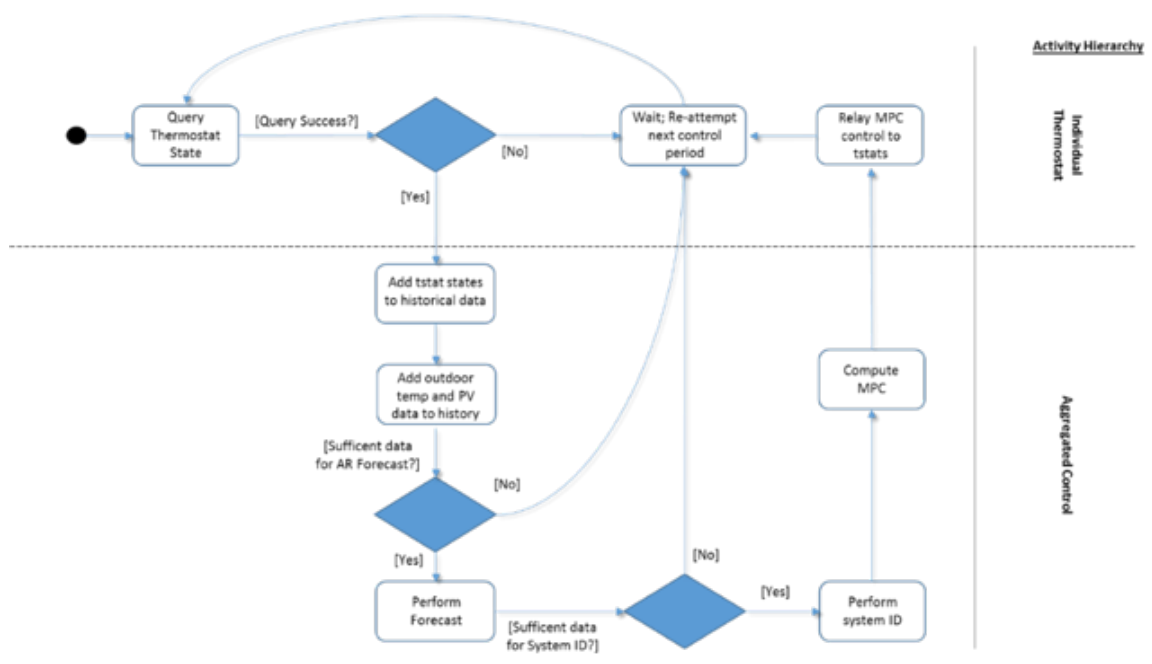

Figure 63. Controller workflow.

Predeployment testing was performed by creating a distinct model outside the controller. Although previous testing attempts operated under the assumption that all information was explicitly known, these simulations were performed by using all developed forecasting and system identification modules. Data were still taken from historical data files, but no information from future time steps was known at the control period. Moving from predeployment testing to field testing will only require functions that draw information from external sources to be replaced with their respective APIs. Figure 64 shows the structure of the experimental setup.

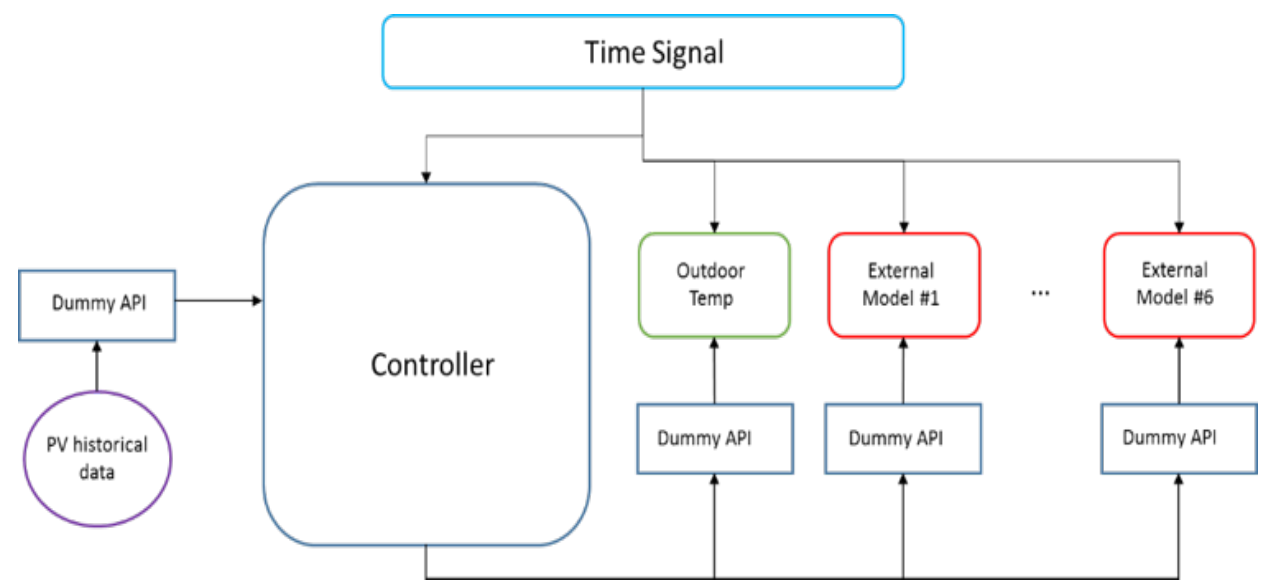

Figure 64. Simulation-based testing experimental setup.

The external model was constructed by taking known $A, B$, and $G$ state-space models and advancing the model in parallel with the control. Six zones were used in this test, mimicking the planned number of zones in field testing. The PV signal that the adaptive control attempts to track was reduced accordingly. Figure 65 shows the results of the testing. With only four units, the system was able to track the PV relatively well. A larger amount of flexible load allows greater precision in tracking the PV signal. 

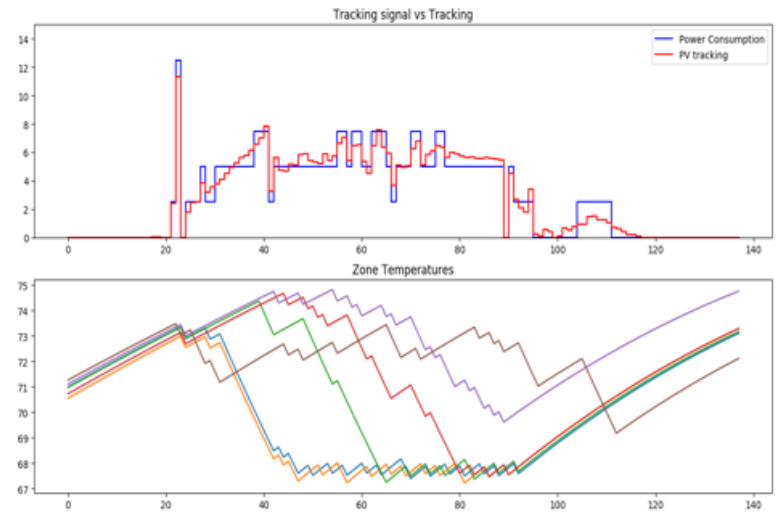

Figure 65. Simulation-based testing PV tracking and temperature data.

An additional test was to determine the effect of noise on the ability to effectively identify the systems in question. In practice, noise could be the effect of local interference at the thermostat. This was tested in simulation by adding noise to the incoming temperature measurements and determining the ability of the controller to identify the system and track the PV. The results of this testing are shown in Figures 66 and 67, and noise within the state measurements disrupts the system identification and impedes the MPC ability to track the PV signal while keeping temperatures within appropriate constraints. To impede this disruption, sufficient fail-safes must be built into the controller to guarantee occupant comfort within certain bands.

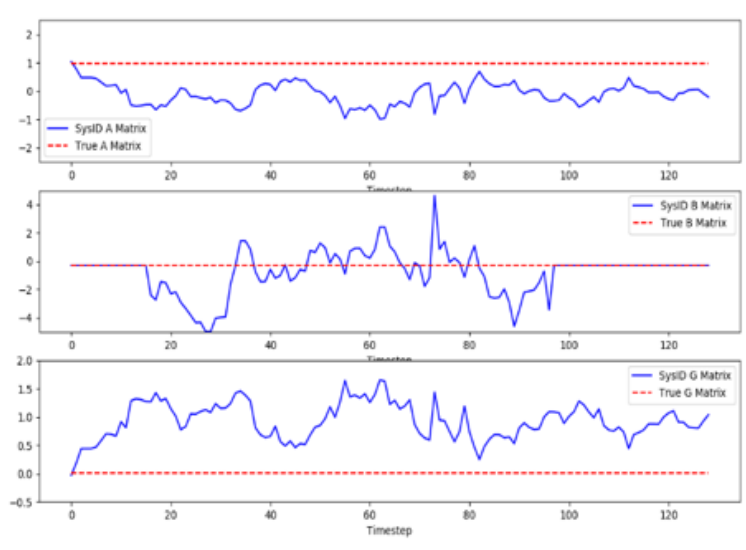

(a)

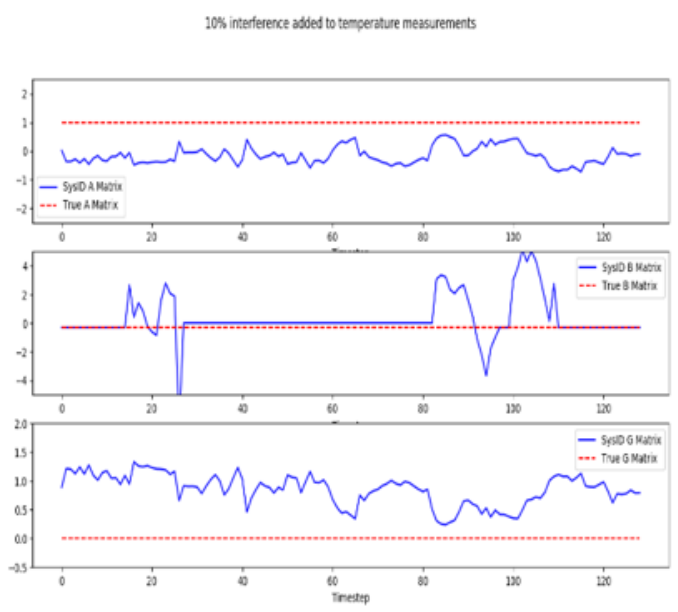

(b)

Figure 66. State-space model estimation via least-square system identification with (a) $5 \%$ noise and (b) $10 \%$ noise. 

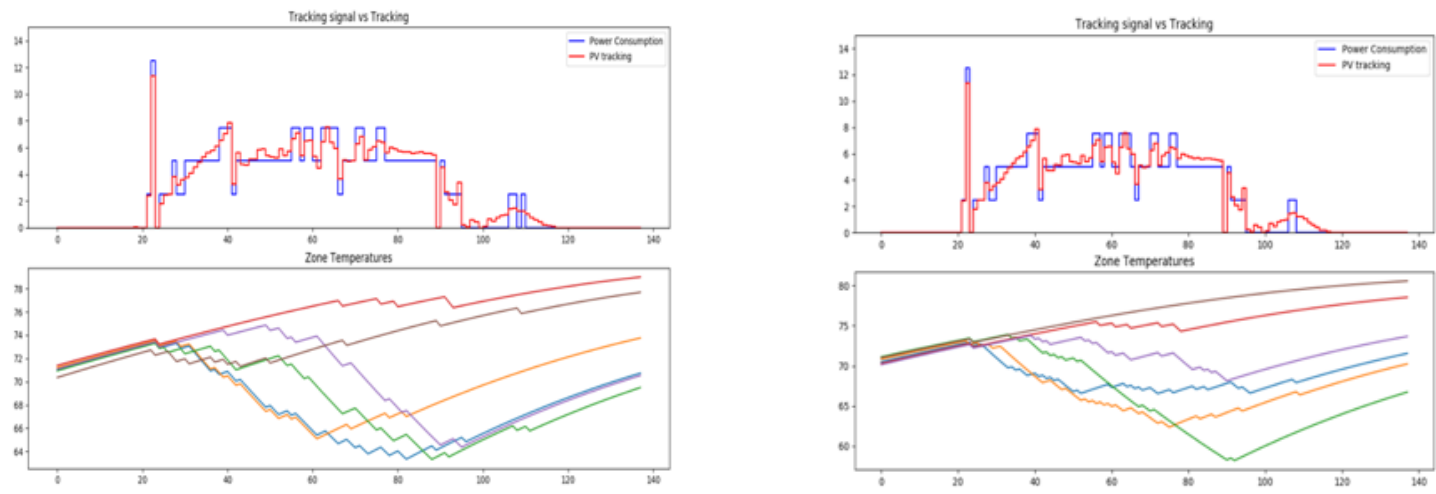

(a)

(b)

Figure 67. PV tracking and temperature measurements with

(a) $5 \%$ noise and (b) $10 \%$ noise.

\subsubsection{Trial 1}

The system was installed, and summer testing is underway. The system was deployed at the Central Baptist Church (CBC) Family Life Center in Fountain City, Tennessee. The units being controlled consist of four 10 ton rooftop HVAC units that cool the basketball court and two 7.5 ton units that cool the racquetball courts. The layout is shown in Figure 68. Although the four 10 ton units have two-stage compressors, the second stage was disabled for the purposes of this study.

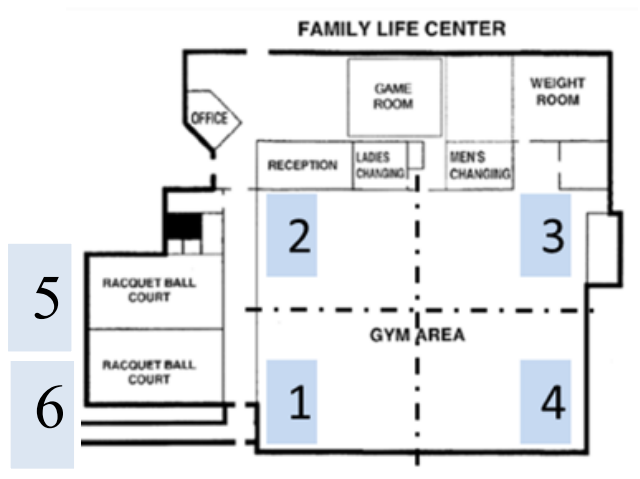

Figure 68. Map of the six zones utilized for MPC testing.

The zones are controlled by six Ecobee 3 smart thermostats mounted on the walls of the gym and protected by plastic enclosures (Figure 69). Thermostat actions are controlled externally through the controlling computer by swinging the set point $3^{\circ} \mathrm{F}$ above or below the current temperature, depending on the desired state of the unit. The unit states are evaluated at every control period of $10 \mathrm{~min}$ to validateand correct, if needed - the MPC forecasting and decisions. 

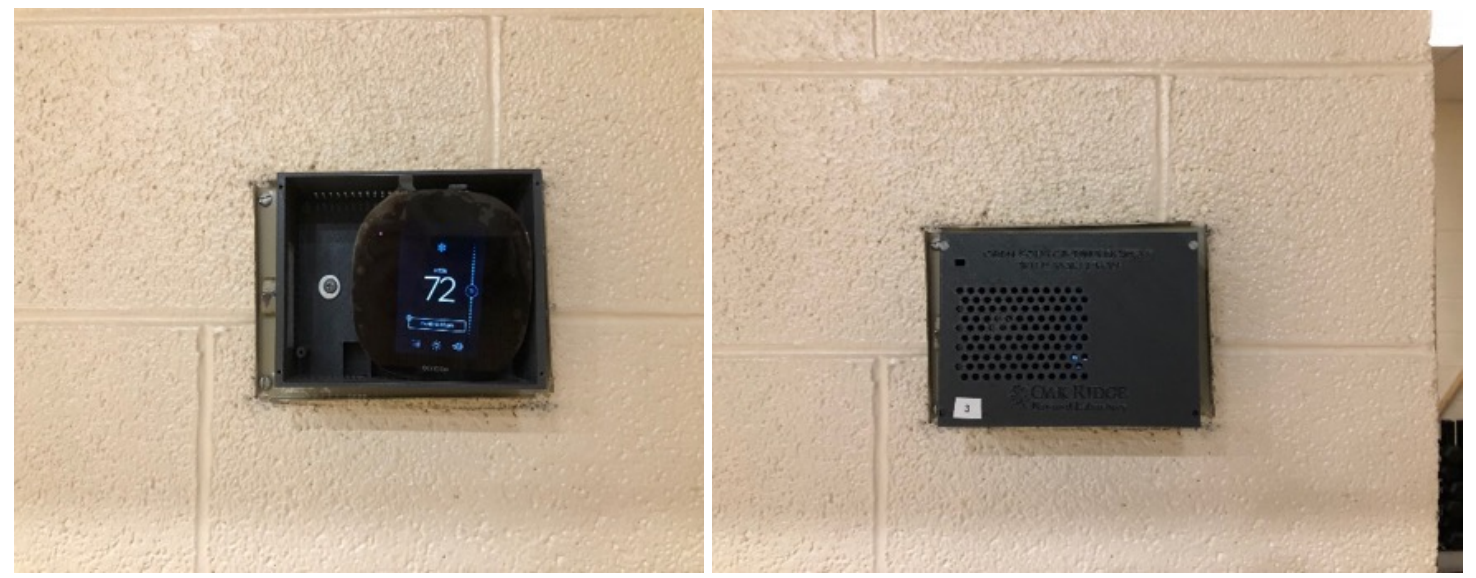

Figure 69. Ecobee thermostat in enclosure (left) with faceplate attached (right).

\section{Test Setup}

The testing apparatus comprised the six thermostats and HVAC units, as well as a set of APIs and historical data used to inform the control. Currently, weather data for the outdoor temperature are taken from the OpenWeather API. AR was then used to generate a forecast for the next hour. PV data were taken from a set of historical data at 10 min intervals. AR was also used to generate a forecast. The controller has no knowledge of the future data that will be drawn from the historical data. In the future, the PV data will be replaced with a live PV measurement. To maintain occupant comfort, the set point for control was set to $71^{\circ} \mathrm{F}$. The control was required to maintain a zone temperature within $3^{\circ} \mathrm{F}$ of the set point, leading to a temperature band of $\left[68^{\circ} \mathrm{F}, 74^{\circ} \mathrm{F}\right]$.

\section{Installation and Testing Process}

After the Ecobee thermostats were deployed but before the decisions from the MPC were allowed to be passed back to the thermostats, the temperature forecasting and ability of the controller to solve the presented control problem were observed. The temperature forecasting (Figure 70) provided an effective projection of the immediate next time step. However, this projection will deviate some as the time between the original forecast and the observed measurement increases. The ability of the control to solve the control problem is shown in Figure 71. Although there are some periods during which the control is unable to solve the problem, this is most likely a result of the initial state being unsuitable. Once the controller is allowed to enforce its control decision, the initial conditions between control periods are expected to stay within a range more suitable for the control throughout the course of the study. 

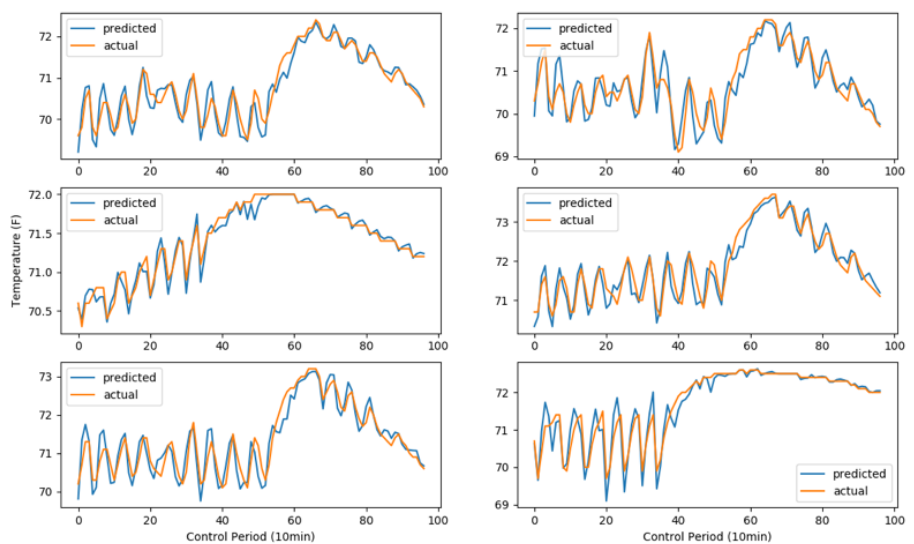

Figure 70. Predicted vs. actual zone temperatures.
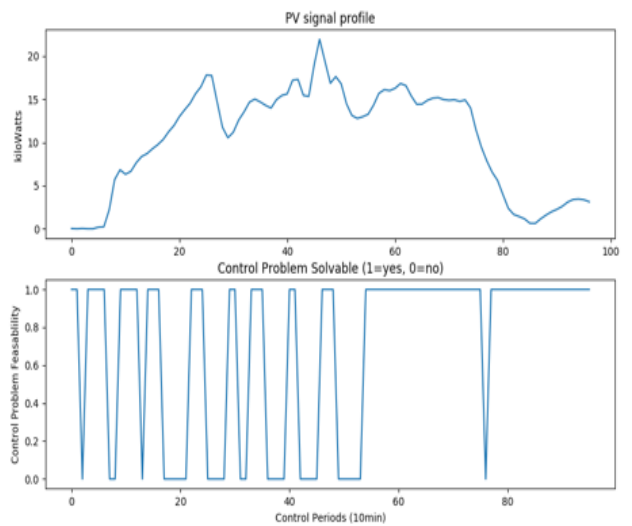

Figure 71. PV signal and control problem feasibility.

\section{Testing Results}

Two tests were undertaken. One was a shorter test that was only $2 \mathrm{~h}$. This test was undertaken to identify and resolve any lingering software bugs. The second was a longer test $(4 \mathrm{~h})$. The results of the first test are shown in Figure 72. In the initial testing, the building energy consumption tracked the PV trajectory well with some slight deviation in magnitude. The second test had slightly reduced performance tracking PV due to some spikes in unit operation (Figure 73). In both sets of testing, the control was able to keep zones within the temperature deadband. The team will continue to work to align the PV tracking more closely. This will primarily be done through modification to the current system identification module. Additionally, the team will seek to add more HVAC units to allow more granular control over the PV tracking. The team also plans to recruit a live PV measurement to align more closely with the envisioned operation of the final system.

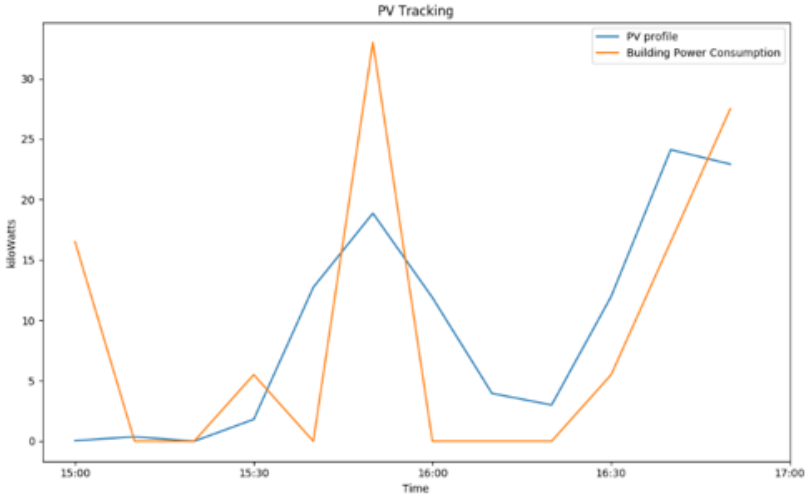

(a)
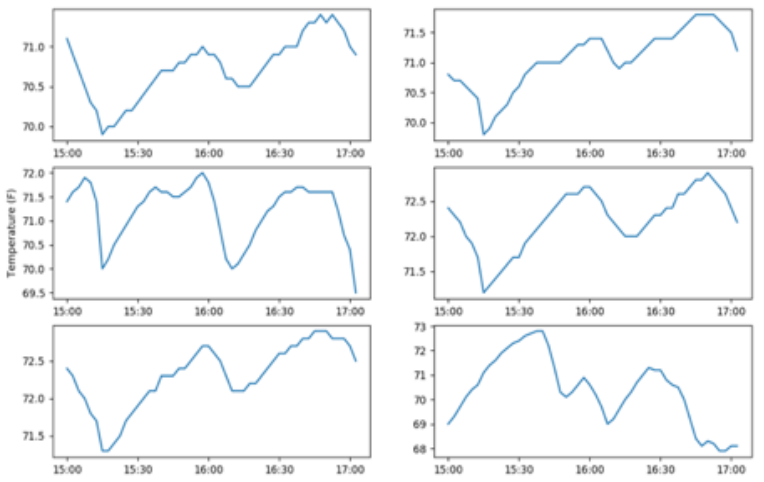

(b)

Figure 72. (a) MPC PV tracking test \#1 and (b) zone temperatures test \#1. 


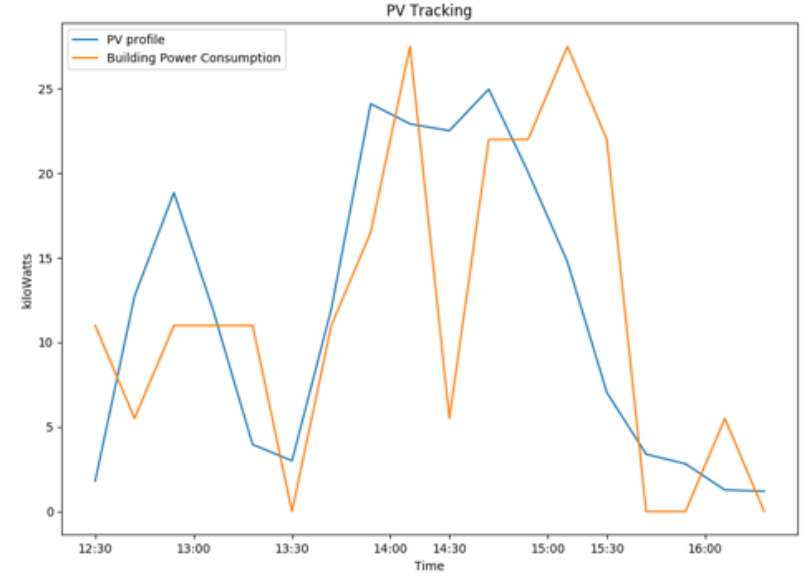

(a)
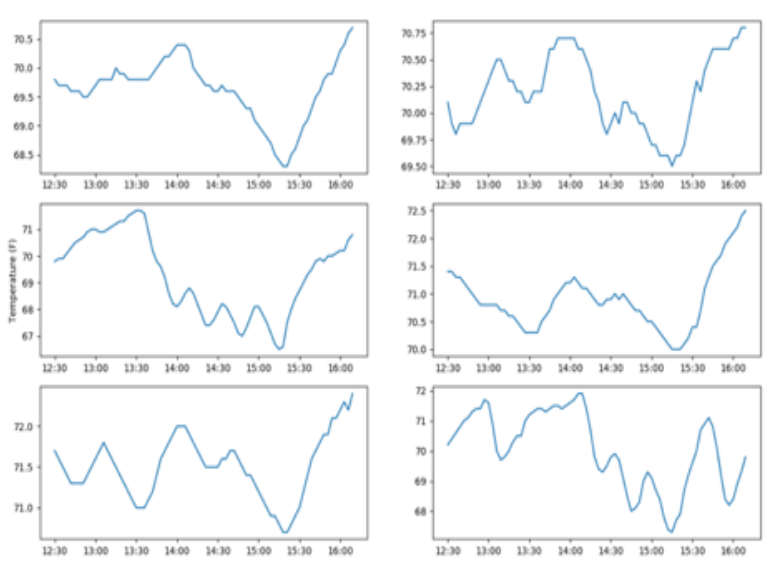

(b)

Figure 73. (a) MPC PV tracking test \#2 and (b) zone temperatures test \#2.

\subsubsection{Trial 2}

\section{Test Setup}

The current testing apparatus comprises the six previously discussed thermostats and HVAC units and a set of APIs and historical data used to inform the control. Currently, weather data for outdoor temperature are taken from the OpenWeather API. The controller has no knowledge of the future data that will be drawn from the historical data. In most tests, to maintain occupant comfort, the set point for control was set to $71^{\circ} \mathrm{F}$. The control was required to maintain a zone temperature within $3^{\circ} \mathrm{F}$ of this set point, leading to a temperature band of $\left[68^{\circ} \mathrm{F}, 74^{\circ} \mathrm{F}\right]$. As testing continued into the autumn months, the set point constraints were slightly relaxed to allow for cooler ambient temperatures. In those cases, set points were set to $69^{\circ} \mathrm{F}$ with a $4^{\circ} \mathrm{F}$ deadband.

The PV profile used in each test was the same and is shown in Figure 74

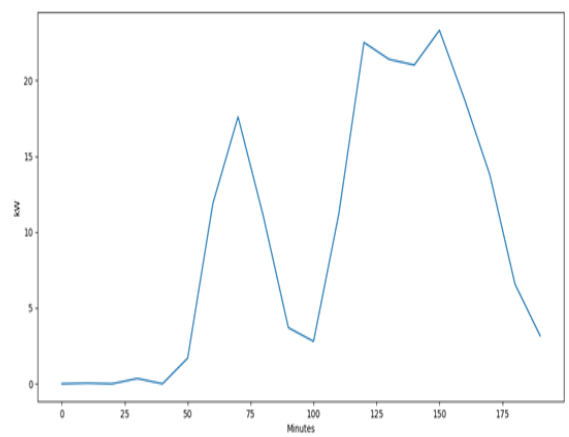

Figure 74. PV ref. signal.

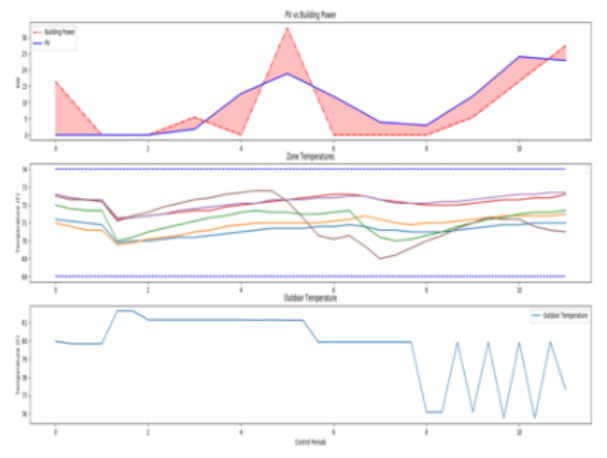

Figure 75. August 10 test. 


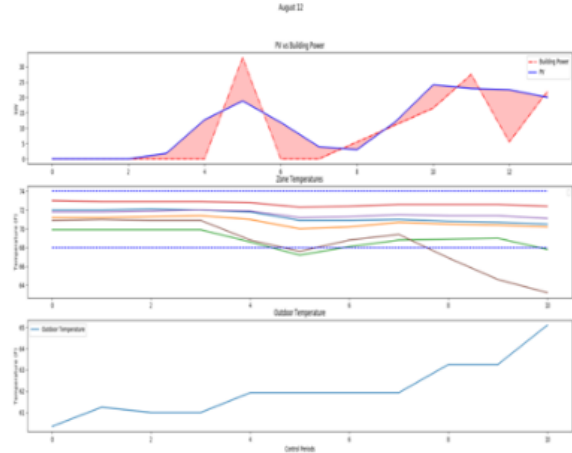

Figure 76. August 12 test.

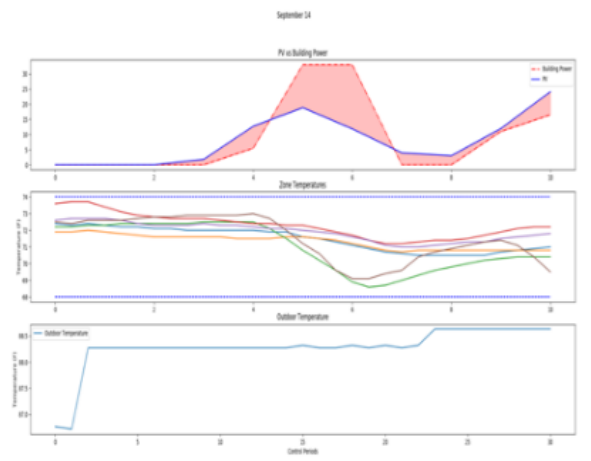

Figure 78. September 14 test.

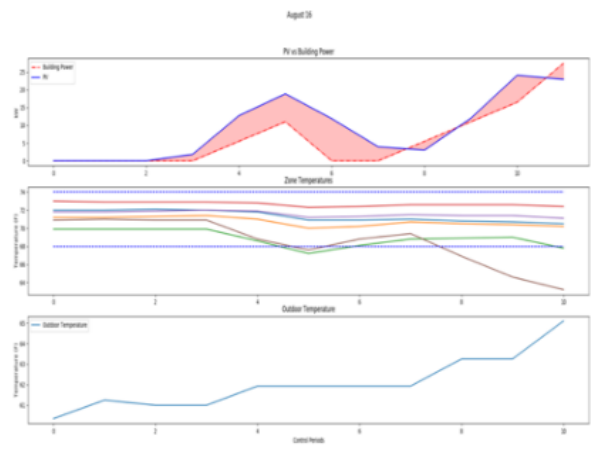

Figure 77. August 16 test.

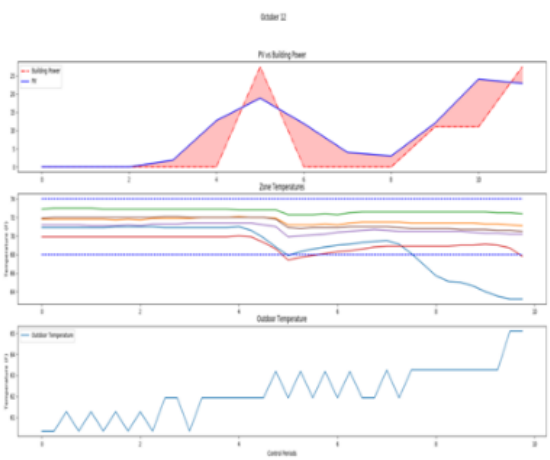

Figure 79. October 12 test.

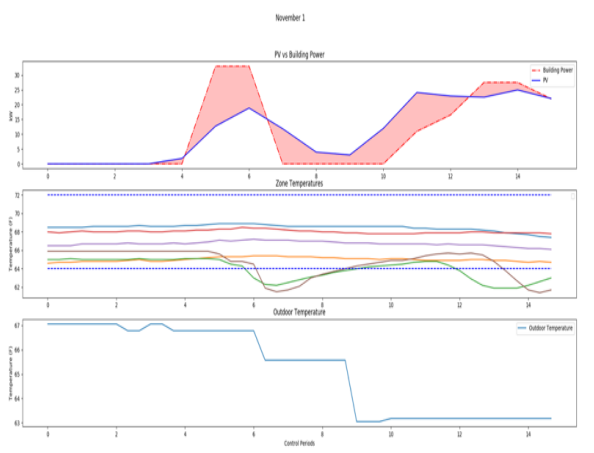

Figure 80. November 1 test.

. The actual power values can be further scaled according to any additional testing with more units. In this specific case, all six units were used to attempt to follow the PV. As the number of units are scaled up, the granularity of the control can also be increased. The RTUs are sub-metered to monitor power. However, due to communications difficulties, for this study, the states of the units were monitored, and power values were calculated by using the number of units known to be on.

\section{Testing Results}

At the time of writing, six tests have been undertaken at different days. Each test was $3-4 \mathrm{~h}$, and the testing timeline was somewhat variable due to building operations. In all cases, the PV tracking was relatively consistent across multiple tests. The results of the six tests are shown in Figures 75-80. 


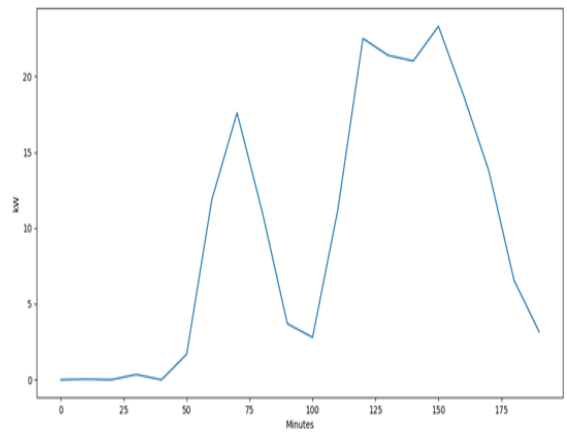

Figure 74. PV ref. signal.

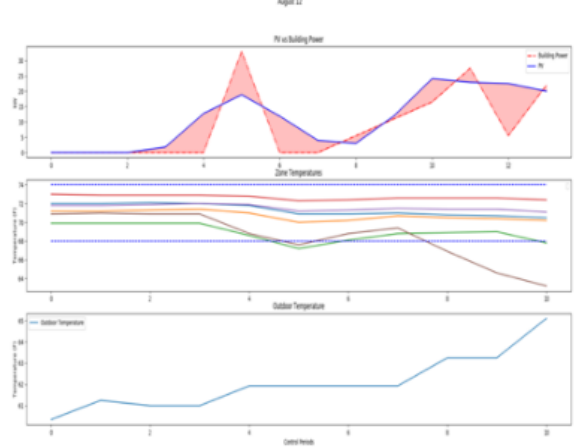

Figure 76. August 12 test.

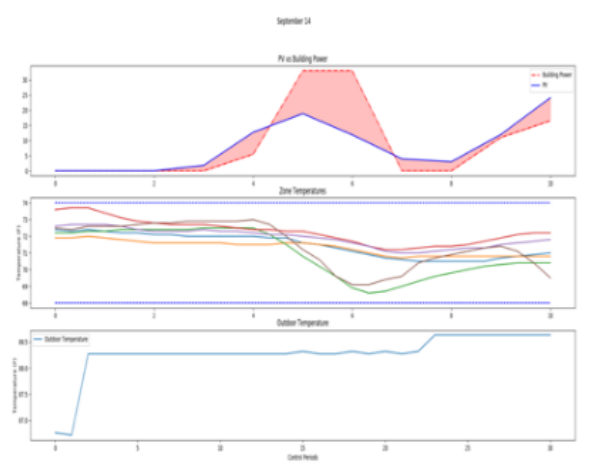

Figure 78. September 14 test.

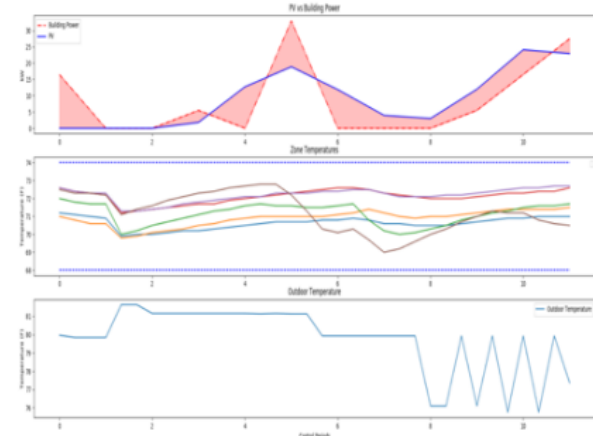

Figure 75. August 10 test.

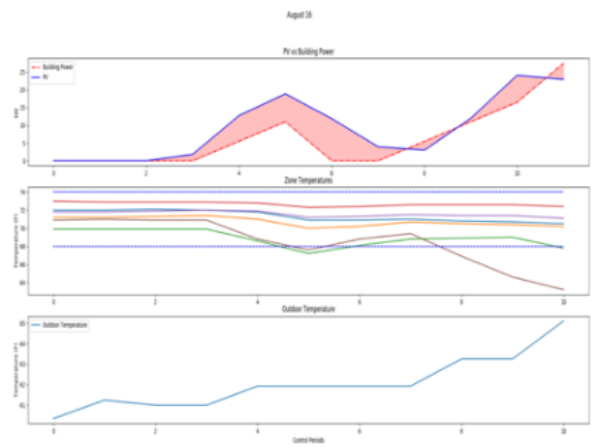

Figure 77. August 16 test.

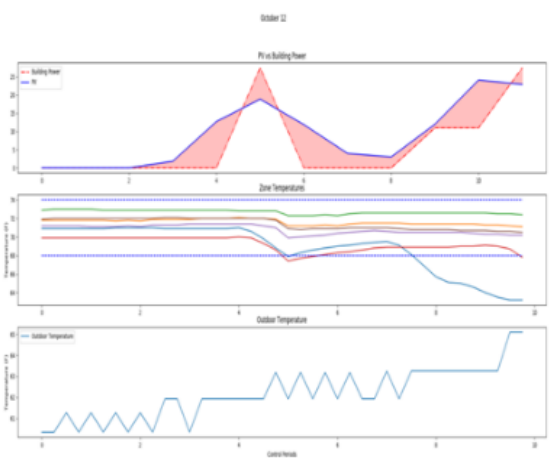

Figure 79. October 12 test.

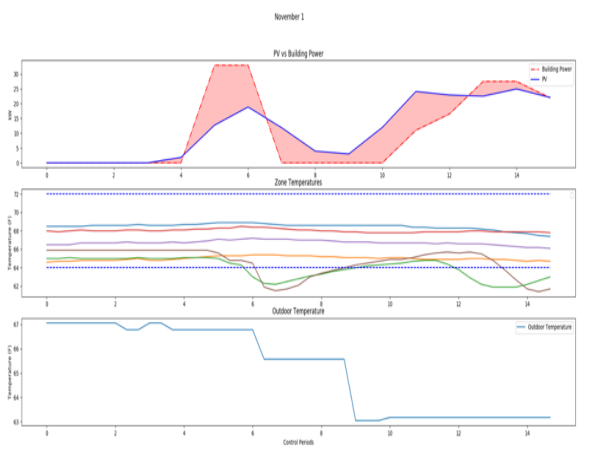

Figure 80. November 1 test. 
Much of the deviation from load shape results from inconsistency between the model used in the control and the actual behavior of the building. Buildings have complex and nonlinear behavior that cannot always be captured by the workings of a first-order resistance-capacitance (RC) model. Additionally, the lag time between a state change and the effect on the monitored space can result in difficulties in forecasting. For example, if a state change is enforced at control period one but the effect is not seen until control period three, then that will obfuscate the calculation of the state variables. As such, more complex and nonlinear models are necessary for accurate forecasting.

The tuning parameters control the priorities of the controller. The controller must strike a balance between following temperature constraints, following the reference signal, and minimizing switching events. A careful study of these parameters might help define an equilibrium among following temperature constraints, following the reference signal, and minimizing switching.

Another test was performed the following spring on the same site (i.e., the six-zone CBC Family Life Center in Fountain City, Tennessee). The site comprised four 10 ton RTUs in a gymnasium and two 7.5 ton units that controlled cooling in racquetball courts. Gymnasium units are two stage; however, only the first stage is used for testing. This option can be set at the Ecobee thermostat. The temperature deadband was set to $70 \pm 3^{\circ} \mathrm{F}$. An additional change to the testing occurred in the system identification step of the optimization module. Previously, the system identification was loosely bounded, relying on the historical data and the least-squares regression to provide an accurate system model. This was changed to a more restrictive bounding problem to ensure that the model provides a conservative estimate of the effects of the cooling. This was done to account for overcooling and for a lag between the system state change and the zone temperature caused by the thermal mass. The results of the test are shown in Figure 81. The temperatures of the zones are show in Figure 82. For most of the test, the MPC controller was able to track with the PV signal with two notable exceptions. The first occurred during the first hour of the test when the PV signal was near zero; however, the units were activated (blue circle). This period was a "ramp up" period during which the controller gathered sufficient historical data. No dispatch occurred during this period. Therefore, the controller could not match the signal. The second point of interest (red circle) occurred during an abrupt change in the PV signal. Previously, the signal was either growing or stable; however, at that point, the PV signal decreased. The forecasting needed to catch up with the decrease, resulting in a lag in the signal tracking.

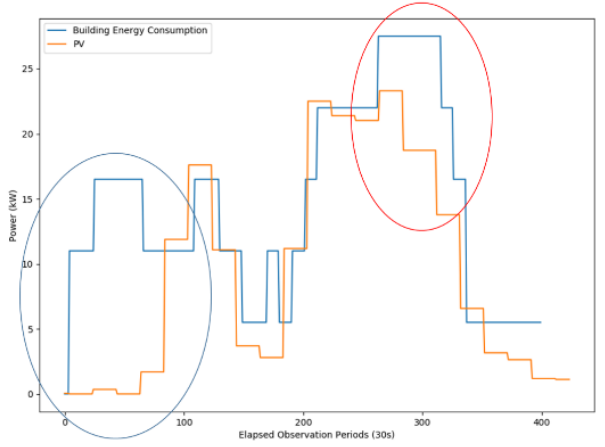

Figure 81. PV tracking results.

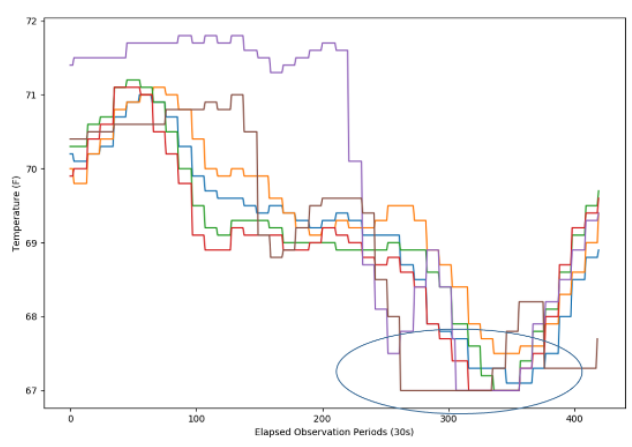

Figure 82. Zone temperatures.

This can be mitigated by better forecasting. For the temperature measurements, there is one point of interest (blue circle). Because of imprecise system identification and the lag induced by thermal mass, the supervisor was required to step in to maintain temperature within the acceptable thermal bound. 
In conclusion, the performance of the MPC was satisfactory in following the general shape of the reference load signals. To more fully test the MPC, more buildings and loads must be recruited to allow for a higher fidelity control response. Furthermore, the model used in the MPC and the forecasting must be refined. An MPC controller can only be as accurate as the model it uses. In the future, more complex models enhanced with machine learning will be able to capture some of the dynamics of large buildings. The software framework has demonstrated the effective deployment of control to enable the increased penetration of solar PVs at the feeder level.

Uncertainty analysis for the different building components and weather forecasts is presented in Appendix A.9.

\section{SIGNIFICANT ACCOMPLISHMENTS AND CONCLUSIONS}

In this project, the team developed and demonstrated: (1) a detailed, simulation-driven understanding of the distribution-level PV integration requirements; (2) an end-to-end simulation-based design of a distributed control framework of loads geographically near the PV generation asset to minimize the effect on the distribution feeder; and (3) control technology deployment to evaluate the effectiveness and to perform scalability analysis at the utility scale.

\section{INVENTIONS, PATENTS, PUBLICATIONS, AND OTHER RESULTS}

\subsection{PUBLICATIONS}

1. L. Bai, Y. Xue, G. Xu, J. Dong, M. Olama, and T. Kuruganti, “A Data-Driven Network Optimisation Approach to Coordinated Control of Distributed Photovoltaic Systems and Smart Buildings in Distribution Systems," IET Energy Systems Integration, June 2021.

2. M. Olama, J. Dong, I. Sharma, Y. Xue, and T. Kuruganti, "Frequency Analysis of Solar PV Power to Enable Optimal Building Load Control,” Energies, vol. 13, no. 18, Article ID 4593, Sep. 2020.

3. J. Dong, M. Olama, T. Kuruganti, A. Melin, S. M. Djouadi, Y. Zhang, and Y. Xue, "Novel Stochastic Methods to Predict Short-term Solar Radiation and Photovoltaic Power," Renewable Energy, vol. 145, pp. 333-346, July 2019.

4. I. Sharma, J. Dong, A. A. Malikopoulos, M. Street, J. Ostrowski, T. Kuruganti, and R. Jackson, “A Modeling Framework for Optimal Energy Management of a Residential Building," Energy and Buildings, vol. 130, pp. 55-63, Oct. 2016.

5. T. Wu, M. Olama, S. M. Djouadi, J. Dong, Y. Xue, and T. Kuruganti, "Signal Temporal Logic Control for Residential HVAC Systems to Accommodate High Solar PV Penetration," in Proc. of the $11^{\text {th }}$ IEEE Conf. on Inn. Smart Grid Tech. (ISGT), Feb. 2020.

6. Y. Zhang, J. Dong, T. Kuruganti, S. Shen, and Y. Xue, "Distributionally Robust Building Load Control to Compensate Fluctuations in Solar Power Generation," in Proc. of the IEEE American Control Conference (ACC'19), July 2019.

7. B. Telsang, M. Olama, S. Djouadi, J. Dong, and T. Kuruganti, "Stability Analysis of Model-free Control under Constrained Inputs for Control of Building HVAC Systems," in Proc. of the IEEE American Control Conference (ACC'19), July 2019.

8. B. Telsang, J. Dong, M. Olama, T. Kuruganti, and S. Djouadi, "Nuclear-norm-based Subspace Identification of Multi-Zone Building HVAC System," in Proc. of the $3^{\text {rd }}$ IEEE International Conference on Smart Grid and Smart Cities (ICSGSC'19), June 2019. 
9. J. Dong, T. Kuruganti, S. M. Djouadi, M. Olama, and Y. Xue, "Privacy-Preserving Aggregation of Controllable Loads to Compensate Fluctuations in Solar Power," in Proc. of the IEEE Workshop on the Electronic Grid (eGrid 2018), Nov. 2018.

10. J. Dong, M. Olama, T. Kuruganti, A. Melin, J. Nutaro, C. Winstead, and Y. Xue, "Model Predictive Control of Building On/Off HVAC Systems to Compensate Fluctuations in Solar Power Generation," in Proc. of the $9^{\text {th }}$ International Conf. on Power Electronics for Distributed Generation Systems (PEDG2018), June 2018. (Best Paper Award)

11. B. Telsang, M. Olama, S. Djouadi, T. Kuruganti, J. Dong, and Y. Xue, "Model-free Temperature Control for Building Air-conditioning Systems to Accommodate Solar Photovoltaic Energy," in Proc. of the $9^{\text {th }}$ International Conference on Power Electronics for Distributed Generation Systems (PEDG2018), Charlotte, NC, June 2018.

12. J. Dong, Y. Xue, T. Kuruganti, M. Olama, and J. Nutaro, "Distribution Voltage Control: Current Status and Future Trends," in Proc. of the $9^{\text {th }}$ International Conference on Power Electronics for Distributed Generation Systems (PEDG2018), Charlotte, NC, June 2018.

13. Y. Xue, M. Starke, J. Dong, M. Olama, T. Kuruganti, J. Taft, and M. Shankar, "On a Future for Smart Inverters with Integrated System Functions," in Proc. of the $9^{\text {th }}$ International Conference on Power Electronics for Distributed Generation Systems (PEDG2018), Charlotte, NC, June 2018.

14. J. Dong, Y. Xue, T. Kuruganti, I. Sharma, J. Nutaro, M. Olama, J. M. Hill, and J. W. Bowen, "Operational Impacts of High Penetration Solar Power on a Real-World Distribution Feeder," in Proc. of the $9^{\text {th }}$ IEEE Inn. Smart Grid Tech. (ISGT), Feb. 2018.

15. Y. Xue, I. Sharma, T. Kuruganti, J. Nutaro, J. Dong, M. Olama, and D. Fugate, "Voltage Impacts Analyses of Solar Photovoltaics on Distribution Load Tap Changer Operations," in Proc. of the $49^{\text {th }}$ North American Power Symposium, Morgantown, WV, Sep. 2017.

16. O. Bara, M. Olama, S. Djouadi, and T. Kuruganti, "Data Driven Building Load Control for High Penetration of Solar Photovoltaic Generation," in Proc. of the $49^{\text {th }}$ IEEE North American Power Symposium, Morgantown, WV, Sep. 2017.

17. J. Dong, S. Djouadi, T. Kuruganti, and M. Olama, "Augmented Optimal Control for Buildings under High Penetration of Solar Photovoltaic Generation," in Proc. of the 2017 IEEE Conference on Control Technology and Applications, Aug. 2017.

18. M. Olama, I. Sharma, T. Kuruganti, J. Dong, J. Nutaro, and Y. Xue "Spectral Analytics of Solar Photovoltaic Power Output for Optimal Distributed Energy Resource Utilization," in Proc. of the 2017 IEEE PES General Meeting, Chicago, IL, July 2017.

19. J. Dong, M. Olama, T. Kuruganti, J. Nutaro, Y. Xue, I. Sharma, and S. Djouadi, “Adaptive Building Load Control to Enable High Penetration of Solar Photovoltaic Generation," in Proc. of the 2017 IEEE PES General Meeting, Chicago, IL, July 2017.

20. J. Dong, T. Kuruganti, A. A. Malikopoulos, S. M. Djouadi, and L. Wang, "Home Energy Management based on Optimal Production Control Scheduling with Unknown Regime Switching," in Proc. of the IEEE American Control Conference (ACC), May 2017.

21. M. Olama, I. Sharma, T. Kuruganti, and D. Fugate, "Statistical Analysis of Solar PV Power Frequency Spectrum for Optimal Employment of Building Loads" in Proc. of the $8^{\text {th }}$ IEEE Conference on Innovative Smart Grid Technologies (ISGT), Apr. 2017.

22. J. Dong, T. Kuruganti, and S. M. Djouadi, "Very Short-term Photovoltaic Power Forecasting using Uncertain Basis Function," in Proc. of the $51^{\text {st }}$ Annual Conference on Information Sciences and Systems (CISS 2017), Mar. 2017. 
23. J. Dong, A. Malikopoulos, S. M. Djouadi, and T. Kuruganti, "Application of Optimal Production Control theory for Home Energy Management in a Micro Grid," in Proc. of the IEEE American Control Conference (ACC), July 2016.

\subsection{INVENTIONS}

1. M.M. Olama, T. Kuruganti, and J. Dong, "Spectral Analysis of Solar Photovoltaic Power Output for Determining Energy Storage System Capacity,” ORNL Invention Disclosure 201804283, DOE S\# S138,954, Jan. 2019.

2. J. Dong, M.M. Olama, T. Kuruganti, and Y. Xue, "Privacy-preserving Aggregation for Smart Metering: Exploiting Randomness in Solar Power Output," ORNL Invention Disclosure 201904305, Jan. 2019. (Elected to be Filed for Patent)

\section{PATH FORWARD}

The team developed and deployed the open-source software for engaging the end-use load to increase renewable penetration at the feeder level. This technology enables the higher penetration of PV resources into distribution circuits and minimizes the impact on the feeder due to two-way power flows. The project advanced the control formulation from the technology readiness level of this technology from 3 to 7 , and it is currently ready for adoption by utilities, building equipment manufacturers, and energy service companies. The team is engaging with utility partners and small business PV installers to demonstrate a seamless transition between the development phase, demonstration phase, and large-scale deployment phase consistent with the long-term objective of the funding agency of using funded research and development programs to promote job creation and economic prosperity within the United States. 


\section{REFERENCES}

[1] SunShot National Laboratory Multiyear Partnership (SuNLaMP) Call for Proposals FY2016FY2018.

[2] J. R. Stitt, "Implementation of a large-scale direct load control system some critical factors," IEEE Trans. App. Syst., vol. PAS-104, no. 7, pp. 1663-1669, 1985.

[3] J. M. Mcintyre, L. Ciecior, A. Kaspar, and D. Castrop, "Distributed intelligence in load control: Results of an experiment using demand limiting devices for residential load control," IEEE Trans. App. Syst., vol. PAS-104, no. 5, pp. 1140-1146, 1985.

[4] Y. Lin, P. Barooah, and S. P. Meyn, "Low-Frequency Power-Grid Ancillary Services from Commercial Building HVAC Systems," in Proc. IEEE International Conference on Smart Grid Communications (SmartGridComm), pp. 169-174, Oct. 2013.

[5] G. Heffner, C. Goldman, B. Kirby, and M. Kintner-Meyer, "Loads providing ancillary services: Review of international experience," Lawrence Berkeley National Laboratory, Berkeley, CA, Tech. Rep., LBNL-62701, ORNL/TM-2007/060, PNNL-16618, 2007.

[6] B. Dunn, H. Kamath, and J.-M. Tarascon, "Electrical energy storage for the grid: A battery of choices," Science, vol. 334, no. 6058, pp. 928-935, 2011.

[7] I. Hiskens and D. Callaway, "Achieving controllability of plug-in electric vehicles," in Proc. IEEE Veh. Power Propulsion Conf. (VPPC ’09), pp. 1215-1220, 2009.

[8] C. Guille, "A conceptual framework for the vehicle-to-grid (V2G) implementation," Ph.D. dissertation, Univ. of Illinois, Urbana-Champaign, IL, USA, 2009.

[9] D. Callaway and I. Hiskens, “Achieving controllability of electric loads," Proc. IEEE, vol. 99, no. 1, pp. 184-199, Jan. 2011.

[10] J. Kondoh, N. Lu, and D. J. Hammerstrom, "An evaluation of the water heater load potential for providing regulation service,” IEEE Trans. Power Syst., vol. 26, no. 3, pp. 1309-1316, Aug. 2011.

[11] D. Hammerstrom, J. Brous, T. Carlon, D. Chassin, C. Eustis, G. Horst, O. Järvegren, R. Kajfasz, W. Marek, P. Michie, R. Munson, T. Oliver, and R. Pratt, "Pacific Northwest GridWise Testbed Demonstration Project. Part II. Grid Friendly Appliances," 2007, Tech. Rep., PNNL report 17079 .

[12] D. S. Callaway, "Tapping the energy storage potential in electric loads to deliver load following and regulation, with application to wind energy," Energ. Convers. Manag., vol. 50, no. 9, pp. 1389-1400, 2009.

[13] N. A. Sinitsyn, S. Kundu, and S. Backhaus, "Safe protocols for generating power pulses with heterogeneous populations of thermostatically controlled loads," Energy Convers. Manage., vol. 67, no. 0, pp. 297-308, 2013.

[14] S. Kundu, N. Sinitsyn, S. Backhaus, and I. Hiskens, "Modeling and control of thermostatically controlled loads," in Proc. Power Syst. Comput. Conf. (PSCC), Stockholm, Sweden, 2011.

[15] C. Perfumo, "Dynamic modelling and control of heterogeneous populations of thermostatically controlled loads," Ph.D. dissertation, Univ. of Newcastle, Callaghan, Australia, 2013.

[16] N. Motegi, M. A. Piette, D. S. Watson, S. Kiliccote, and P. Xu, "Introduction to commercial building control strategies and techniques for demand response," Tech. Rep., LBNL report 59975, 2007. 
[17] J. Mathieu, S. Koch, and D. Callaway, "State estimation and control of electric loads to manage real-time energy imbalance," IEEE Trans. Power Syst., vol. 28, no. 1, pp. 430-440, Feb. 2013

[18] G. Goddard, J. Klose, and S. Backhaus, "Model Development and Identification for Fast Demand Response in Commercial HVAC Systems," IEEE Transactions on Smart Grid, vol. 5, no. 4, pp. 2084-2092, July 2014.

[19] P. Zhao, G. P. Henze, M. J. Brandemuehl, V. J. Cushing, and S. Plamp, "Dynamic frequency regulation resources of commercial buildings through combined building system resources using a supervisory control methodology," Energy and Buildings, vol. 86, Jan. 2015.

[20] J. L. Mathieu, M. Dyson, and D. S. Callaway, "Using Residential Electric Loads for Fast Demand Response: The Potential Resource and Revenues, the Costs, and Policy Recommendations," ACEEE Summer Study on Energy Efficiency in Buildings, 2012

[21] J. L. Mathieu, D. S. Callaway, and S. Kiliccote, "Variability in automated responses of commercial buildings and industrial facilities to dynamic electricity prices," Energy and Buildings, vol. 43, no. 12, pp. 3322-3330, 2011.

[22] N. Lu, "An Evaluation of the HVAC Load Potential for Providing Load Balancing Service," IEEE Transactions on Smart Grid, vol. 3, no. 3, pp. 1263-1270, Sept. 2012. doi: 10.1109/TSG.2012.2183649.

[23] S. Parkinson, D. Wang, C. Crawford, and N. Djilali, "Comfort-constrained distributed heat pump management," in Proc. IEEE Intersoc. Electr. Electron. Eng. (ICSGCE 2011), Piscataway, NJ, 2011.

[24] D. Wang, S. Parkinson, W. Miao, H. Jia, C. Crawford, and N. Djilali, "Online voltage security assessment considering comfort-constrained demand response control of distributed heat pump systems,” Appl. Energy, 2012.

[25] S. Katipamula and N. Lu, "Evaluation of residential HVAC control strategies for demand response programs," ASHRAE Trans., vol. 1, no. 12, pp. 1-12, 2006.

[26] J. H. Yoon, R. Bladick, and A. Novoselac, "Demand response for residential buildings based on dynamic price of electricity," Energy and Buildings, vol. 80, pp. 531-541, Sep. 2014.

[27] D. S. Watson, N. Matson, J. Page, S. Kiliccote, M. A. Piette, K. Corfee, B. Seto, R. Masiello, J. Masiello, L. Molander, S. Golding, K. Sullivan, W. Johnson, and D. Hawkins, "Fast Automated Demand Response to Enable the Integration of Renewable Resources," California Energy Commission. Available at: http://drrc.lbl.gov/sites/all/files/LBNL-5555E.pdf

[28] Accommodating High Levels of Variable Generation, NERC Report, April 2009.

[29] S. J. Steffel, P. R. Caroselli, A. M. Dinkel, J. Q. Liu, R. N. Sackey, and N. R. Vadhar, "Integrating Solar Generation on the Electric Distribution Grid," IEEE Transactions on Smart Grid, vol. 3, no. 2, pp. 878-886, June 2012.

[30] R. Tonkoski, D. Turcotte, and T. H. M. EL-Fouly, "Impact of High PV Penetration on Voltage Profiles in Residential Neighborhoods," IEEE Transactions on Sustainable Energy, vol. 3, no. 3, pp. 518-527, July 2012.

[31] R. Tonkoski, L. A. C. Lopes, and T. H. M. El-Fouly, "Coordinated Active Power Curtailment of Grid Connected PV Inverters for Overvoltage Prevention," IEEE Transactions on Sustainable Energy, vol. 2, no. 2, pp. 139-147, April 2011.

[32] M. J. E. Alam, K. M. Muttaqi, and D. Sutanto, "Mitigation of Rooftop Solar PV Impacts and Evening Peak Support by Managing Available Capacity of Distributed Energy Storage Systems," IEEE Transactions on Power Systems, vol. 28, no. 4, pp. 3874-3884, Nov. 2013. 
[33] F. Olivier, P. Aristidou, D. Ernst, and T. Van Cutsem, "Active Management of Low-Voltage Networks for Mitigating Overvoltages Due to Photovoltaic Units," IEEE Transactions on Smart Grid, vol. 7, no. 2, pp. 926-936, Mar. 2016.

[34] R. Kabiri, D. Holmes, B. McGrath, and L. Meegahapola, "LV grid voltage regulation using transformer electronic tap changing, with PV inverter reactive power injection," IEEE JESTPE, vol. 3, no. 4, pp/ 1182-1192, Dec. 2015.

[35] B. Boashash, Time-frequency signal analysis and processing: A comprehensive reference, Oxford: Elsevier Science, 2003.

[36] A. V. Oppenheim and R. W. Schafer, Discrete-Time Signal Processing, Prentice-Hall, Englewood Cliffs, NJ, pp. 713-718, 1989.

[37] S. Haykin, Advances in Spectrum Analysis and Array Processing, vol. 1, Prentice-Hall, 1991.

[38] R. N. Bracewell, The Fourier Transform and Its Applications, $3^{\text {rd }}$ Edition, Boston: McGraw-Hill, 2000 .

[39] R. McGill, J. W. Tukey, and W. A. Larsen, "Variations of boxplots," The American Statistician, vol. 32, pp. 12-16, 1978.

[40] I. Sharma, J. Dong, A. Malikopoulos, M. Street, J. Ostrowski, T. Kuruganti, and R. Jackson, “A modeling framework for optimal energy management of a residential building," Energy and Buildings, vol. 130, pp. 55-63, Oct. 2016.

[41] G. O. Cimuca, C. Saudemont, B. Robyns, and M. Radulescu, "Control and performance evaluation of a flywheel energy-storage system associated to a variable-speed wind generator," IEEE Trans. Ind. Electron., vol. 53, no. 4, pp. 1074-1085, Jun. 2006.

[42] Energy Information Administration, 2003 Commercial buildings energy consumption survey. Washington, DC: US Energy Information Administration, 2003. Available at www.eia.gov/consumption/commercial/data/2003/. Last accessed April 2016.

[43] B. Fricke, T. Kuruganti, J. Nutaro, D. Fugate, and J. Sanyal, "Utilizing thermal mass in refrigerated display cases to reduce peak demand," in Proc. of the $16^{\text {th }}$ International Refrigeration and Air Conditioning Conference, vol. 2449, 8 pages, 2016.

[44] L. Cheng, Y. Chang, and R. Huang, "Mitigating voltage problem in distribution system with distributed solar generation using electric vehicles," IEEE Trans. Sustain. Energy, vol. 6, no. 4, pp. 1475-1484, Oct. 2015.

[45] P. M. S. Carvalho, P. F. Correia, and L. A. F. M. Ferreira, "Distributed reactive power generation control for voltage rise mitigation in distribution networks," IEEE Trans. Power Syst., vol. 23, no. 2, pp. 766-772, May 2008.

[46] F. Ding, A. Pratt, T. Bialek and F. Bell, et al, "Voltage support study of smart PV inverters on a high-photovoltaic penetration utility distribution feeder," IEEE $43^{\text {rd }}$ Photovoltaic Specialists Conference (PVSC), Portland, OR, June 2016.

[47] P. Jahangiri and D. C. Aliprantis, "Distributed Volt/Var Control by PV Inverters," IEEE Transactions on Power Systems, vol. 28, no. 3, pp. 3429-3439, Aug. 2013.

[48] K. Turitsyn, P. Sulc, S. Backhaus and M. Chertkov, "Options for Control of Reactive Power by Distributed Photovoltaic Generators," Proc. of the IEEE, vol. 99, no. 6, pp. 1063-1073, June 2011. 
[49] M. Farivar, R. Neal, C. Clarke, and S. Low, "Optimal inverter VAR control in distribution systems with high PV penetration," in Proc. IEEE PES GM, San Diego, CA, USA, pp. 1-7, July 2012.

[50] H. Liu, W. Shi, and H. Zhu, "Distributed voltage control in distribution networks: Online and robust implementations," IEEE Trans Smart Grid, vol. 9, no. 6, pp. 6106-6117, Nov. 2018.

[51] P. Sulc, S. Backhaus, and M. Chertkov, "Optimal distributed control of reactive power via the alternating direction method of multipliers," IEEE Trans. Energy Convers., vol. 29, no. 4, pp. 968-977, Dec. 2014.

[52] W. Zheng, W. Wu, B. Zhang, H. Sun, and Y. Liu, "A fully distributed reactive power optimization and control method for active distribution networks," IEEE Trans. Smart Grid, vol. 7, no. 2, pp. 1021-1033, Mar. 2016.

[53] OpenDSS Program, [Online] Available through SourceForge.net, http://sourceforge.net/projects/electricdss.

[54] M. Farivar and S. H. Low, "Branch flow model: Relaxations and convexification-Part I," IEEE Trans. Power Syst., vol. 28, no. 3, pp. 2554-2564, Aug. 2013.

[55] M. Farivar and S. H. Low, "Branch flow model: Relaxations and convexification-Part II," IEEE Trans. Power Syst., vol. 28, no. 3, pp. 2565-2572, Aug. 2013.

[56] S. Boyd, N. Parikh, and E. Chu, "Distributed optimization and statistical learning via the alternating direction method of multipliers," Found. Trends Mach. Learn., vol. 3, no. 1, pp. 1$122,2011$.

[57] “IBM ILOG CPLEX. ver. 12.7,” http://www01.ibm.com/software/integration/optimization/cplex-optimizer.

[58] M. E. Baran and F. F. Wu, "Optimal capacitor placement on radial distribution systems," IEEE Trans. Power Del., vol. 4, no. 1, pp. 725-734, Jan. 1989.

[59] M. Gwerder, "Predictive control for integrated room automation," in Proc. $8^{\text {th }}$ REHVA World Congress, 2005.

[60] X. J. Ma, "Stochastic control of energy efficient buildings: A semidefinite programming approach," in Proc. IEEE International Conference on Smart Grid Communications (SmartGridComm), 2015.

[61] I. G. Optimization, Gurobi optimizer reference manual, 2015. Retrieved from http://www.gurobi.com

[62] J. Lofberg, "YALMIP: A toolbox for modeling and optimization in MATLAB," in Proc. IEEE International Symposium on Computer Aided Control Systems Design, 2004.

[63] PICOS: http://picos.zib.de/index.html.

[64] ZIBOPT: http://scip.zib.de/

[65] PuLP: https://pythonhosted.org/PuLP/

[66] COIN-OR: https://en.wikipedia.org/wiki/COIN-OR

[67] Best Research-Cell Efficiencies, NREL, 2015. Available at: https://www.nrel.gov/pv/cellefficiency.html.

[68] T. Esram and P. L. Chapman, "Comparison of photovoltaic array maximum power point tracking techniques,” IEEE Trans. Energy Convers., vol. 22, no. 2, pp. 439-449, Jun. 2007. 
[69] H. J. El-Khozondar, R. J. El-Khozondar, K. Matter, and T. Suntio, “A review study of photovoltaic array maximum power tracking algorithms," Renewables, 2016.

[70] Y. Xue, K.C. Divya, G. Griepentrog, M. Liviu, and M. Manjrekar, "Towards next generation photovoltaic inverters," in Proc. 2011 IEEE Energy Conversion Congress and Exposition (ECCE), Phoenix, Arizona, USA, pp. 2467-2474, Sep. 2011.

[71] Y. Xue, L. Chang, S. B. Kjaer, J. Bordonau, and T. Shimizu, "Topologies of single-phase inverters for small distributed power generators: An overview," IEEE Trans. Power Electron., vol. 19, pp. 1305-1314, Sep. 2004.

[72] S. B. Kjaer, J. K. Pedersen, and F. Blaabjerg, "A review of single-phase grid connected inverters for photovoltaic modules,” IEEE Trans. Ind. Appl., vol. 41, no. 5, pp. 1292-1306, Sep. 2005.

[73] R. Mechouma, B. Azoui, and M. Chaabane, "Three-phase grid connected inverter for photovoltaic systems: A review," in Proc. First International Conference on Renewable Energies and Vehicular Technology, pp. 37-42, 2012.

[74] G. R. Walker and P. C. Sernia, "Cascaded DC-DC converter connection of photovoltaic modules," IEEE Trans. Power Electron., vol. 19, no. 4, pp. 1130-1139, Jul. 2004.

[75] L. Liu, H. Li, Y. Xue and W. Liu, "Reactive Power Compensation and Optimization Strategy for Grid-Interactive Cascaded Photovoltaic Systems," in IEEE Transactions on Power Electronics, vol. 30, no. 1, pp. 188-202, Jan. 2015.

[76] B. Xiao, L. Hang, J. Mei, C. Riley, L. M. Tolbert, and B. Ozpineci, "Modular cascaded H-bridge multilevel PV inverter with distributed MPPT for grid-connected applications," IEEE Trans. Ind. Appl., vol. 51, no. 2, pp. 1722-1731, Mar./Apr. 2015.

[77] P. S. Shenoy, K. A. Kim, B. B. Johnson, and P. T. Krein, "Differential power processing for increased energy production and reliability of photovoltaic systems," IEEE Trans. Power Electron., vol. 28, no. 6, pp. 2968-2979, June 2013.

[78] R. Bell and R. C. N. Pilawa-Podgurski, "Decoupled and distributed maximum power point tracking of series-connected photovoltaic submodules using differential power processing," IEEE Journal of Emerging and Selected Topics in Power Electronics, vol. 3, no. 4, pp. 881-891, Dec. 2015.

[79] S. W. H. de Haan, H. Oldenkamp, and E. J. Wildenbeest, "Test results of a $130 \mathrm{~W}$ AC module: A modular solar AC power station," 1994 IEEE First World Conference on Photovoltaic Energy Conversion, Conference Record of the Twenty Fourth IEEE Photovoltaic Specialists Conference, Waikoloa, HI, vol.1, pp. 925-928, 1994.

[80] R. H. Wills, S. Krauthamer, A. Bulawka, and J. P. Posbic, "The AC photovoltaic module concept," Energy Conversion Engineering Conference, 1997. IECEC-97, Proceedings of the 32nd Intersociety, Honolulu, HI, vol.3, pp. 1562-1563, 1997.

[81] H. Zhou, J. Zhao and Y. Han, "PV balancers: Concept, architectures, and realization," IEEE Transactions on Power Electronics, vol. 30, no. 7, pp. 3479-3487, July 2015.

[82] L. Chen, A. Amirahmadi, Q. Zhang, N. Kutkut, and I. Batarseh, "Design and implementation of three-phase two-stage grid-connected module integrated converter," in IEEE Transactions on Power Electronics, vol. 29, no. 8, pp. 3881-3892, Aug. 2014.

[83] F. Wang, X. Wu, F. C. Lee, Z. Wang, P. Kong, and F. Zhuo, “Analysis of unified output MPPT control in subpanel PV converter system," IEEE Transactions on Power Electronics, vol. 29, no. 3, pp. 1275-1284, Mar. 2014. 
[84] C. Olalla, D. Clement, M. Rodriguez, and D. Maksimovic, "Architectures and control of submodule integrated DC-DC converters for photovoltaic applications," IEEE Transactions on Power Electronics, vol. 28, no. 6, pp. 2980-2997, June 2013.

[85] Analysis of Extremely Reliable Power Delivery Systems: A Proposal for Development and Application of Security, Quality, Reliability, and Availability (SQRA) Modeling for Optimizing Power System Configurations for the Digital Economy, EPRI, Palo Alto, CA, 2002.

[86] J. D. Bouford and C. A. Warren, "Many states of distribution," IEEE Power and Energy Magazine, vol. 5, no. 4, pp. 24-32, July-Aug. 2007.

[87] Secondary Network Distribution Systems Background and Issues Related to the Interconnection of Distributed Resources, Technical Report, NREL/TP-560-38079, 2005.

[88] R. Das et al., "Distribution automation strategies: Evolution of technologies and the business case,” IEEE Transactions on Smart Grid, vol. 6, no. 4, pp. 2166-2175, 2015.

[89] Flexible and Distributed Energy Resources, Chapter 3: Technology Assessments, DOE Quadrennial Technology Review, 2015.

[90] D. Ranamuka, A. P. Agalgaonkar, and K. M. Muttaqi, "Examining the interactions between DG units and voltage regulating devices for effective voltage control in distribution systems," IEEE Transactions on Industry Applications, vol. 53, no. 2, 2017.

[91] A. T. Procopiou and L. F. Ochoa, "Voltage control in PV-rich LV networks without remote monitoring," IEEE Transactions on Power Systems, vol. 32, no. 2, Mar. 2017.

[92] Grid Strategy 2011: Conservation Voltage Reduction and Volt VAR Optimization in the Smart Grid, EPRI Technical Report 1024482, Nov. 2011.

[93] H. E. Farag, E. F. El-Saadany, and R. Seethapathy, "The evolution for voltage and reactive power control in smart distribution systems," International Journal of Emerging Electric Power Systems, vol. 13, no. 1, article 6, 2012.

[94] M. Madrigal, R. Uluski, and K. M. Gaba, "Practical guidance for defining a smart grid modernization strategy - the case of distribution," The World Bank Group, 2017.

[95] M. Manbachi et al., "Real-time adaptive VVO/CVR topology using multi-agent system and IEC 61850-based communication protocol," IEEE Transactions on Sustainable Energy, vol. 5, no. 2, pp. 587-597, Apr. 2014.

[96] R. Moghe, D. Divan, D. Lewis, and J. Schatz, "Turning distribution feeders into STATCOMs," IEEE Transactions on Industry Applications, vol. 53, no. 2, pp. 1372-1380, March-April 2017.

[97] G. Fusco and M. Russo, Adaptive Voltage Control in Power Systems - Modeling, Design and Applications, Springer, 2006.

[98] V. Khadkikar, "Enhancing electric power quality using UPQC: A comprehensive overview," IEEE Transactions on Power Electronics, vol. 27, no. 5, pp. 2284-2297, May 2012.

[99] Y. W. Li and J. He, "Distribution system harmonic compensation methods: An overview of DGinterfacing inverters," IEEE Industrial Electronics Magazine, vol. 8, no. 4, pp. 18-31, Dec. 2014.

[100] J. Dixon, L. Moran, J. Rodriguez, and R. Domke, "Reactive power compensation technologies: State-of-the-art review," Proc. of the IEEE, vol. 93, no. 12, pp. 2144-2164, Dec. 2005.

[101] Y. Xue, M. Manjrekar, C. Lin, M. Tamayo, and N. Jiang, "Voltage stability and sensitivity analysis of grid-connected photovoltaic systems," Proc. 2011 IEEE Power and Energy Society General Meeting, Detroit, Michigan, USA, pp. 1-7, July 2011. 
[102] Y. Xue and J.M. Guerrero, "Smart inverters for utility and industry applications," Proc. 2015 Power Conversion and Intelligent Motion (PCIM) Europe, Nuremberg, Germany, pp. 277-284, May 2015.

[103] IEEE P1547/D5.0, Draft Standard for Interconnection and Interoperability of Distributed Energy Resources with associated Electric Power Systems Interfaces, August 2016.

[104] Y. P. Agalgaonkar, B. C. Pal, and R. A. Jabr, "Distribution voltage control considering the impact of PV generation on tap changers and autonomous regulators," IEEE Transactions on Power Systems, vol. 29, no. 1, pp. 182-192, Jan. 2014.

[105] I. Kim, R. G. Harley, R. Regassa, and Y. D. Valle, "The effect of the volt/var control of photovoltaic systems on the time-series steady-state analysis of a distribution network," 2015 Clemson University Power Systems Conference, Clemson, SC, pp. 1-6, 2015.

[106] S. Satsangi and G. B. Kumbhar, "Review on Volt/Var optimization and control in electric distribution system," 2016 IEEE 1st International Conference on Power Electronics, Intelligent Control and Energy Systems (ICPEICES), Delhi, pp. 1-6, 2016.

[107] X. Luo, Z. Akhtar, C. K. Lee, B. Chaudhuri, S. C. Tan, and S. Y. R. Hui, "Distributed voltage control with electric springs: Comparison with STATCOM," IEEE Transactions on Smart Grid, vol. 6, no. 1, pp. 209-219, Jan. 2015.

[108] J. C. Das, "Application of STATCOM to an industrial distribution system connected to a weak utility system," IEEE Transactions on Industry Applications, vol. 52, no. 6, pp. 5345-5354, Nov.Dec. 2016.

[109] H. Liao and J. V. Milanović, "On capability of different FACTS devices to mitigate a range of power quality phenomena," IET Generation, Transmission \& Distribution, vol. 11, no. 5, pp. 1202-1211, Mar. 2017.

[110] C. Zhang, Y. Xu, Z. Y. Dong, and J. Ravishankar, "Three-stage robust inverter-based voltage/var control for distribution networks with high-level PV," IEEE Transactions on Smart Grid, vol. 10, no. 1, pp. 782-793, Jan. 2019.

[111] M. Hasheminamin, V.G. Agelidis, V. Salehi, R. Teodorescu, and B. Hredzak, "Index-based assessment of voltage rise and reverse power flow phenomena in a distribution feeder under high PV penetration," IEEE Journal of Photovoltaics, vol. 5, no. 4, pp. 1158-1168, July 2015.

[112] R.J. Broderick et al., "Time series power flow analysis for distribution connected PV generation," Sandia Report, SAND2013-0537, Jan. 2013.

[113] IEEE 13-Node Test Feeder, IEEE Power Engineering Society, Distribution System Analysis Subcommittee.

[114] A. Q. Huang, "Medium-voltage solid-state transformer: Technology for a smarter and resilient grid," IEEE Industrial Electronics Magazine, vol. 10, no. 3, pp. 29-42, Fall 2016.

[115] H. Chen, A. R. Iyer, R. G. Harley, and D. Divan, "Dynamic grid power routing using controllable network transformers (CNTs) with decoupled closed-loop controller," IEEE Transactions on Industry Applications, vol. 51, no. 3, pp. 2361-2372, May-June 2015.

[116] D. Divan, R. Moghe, and A. Prasai, "Power electronics at the grid edge: The key to unlocking value from the smart grid," IEEE Power Electronics Magazine, vol. 1, no. 4, pp. 16-22, Dec. 2014.

[117] J. Carpentier, "Contribution á l'étude du dispatching économique," Bulletin de la Société Française des Électriciens, ser. 8, vol. 3, pp. 431-447, 1962. 
[118] J. Carpentier, "Optimal power flows," International Journal of Electrical Power and Energy Systems, vol. 1, no. 1, pp. 3-15, Apr. 1979.

[119] N. Li, G. Qu and M. Dahleh, "Real-time decentralized voltage control in distribution networks," Proc. of the 52nd Annual Allerton Conference (Allerton), Monticello, IL, pp. 582-588, 2014.

[120] S. Bolognani, G. Cavraro, and S. Zampieri, "Performance analysis of a distributed algorithm for dynamic reactive power compensation," 2012 IEEE 51st IEEE Conference on Decision and Control (CDC), Maui, HI, pp. 296-301, 2012.

[121] K. Zhang and H. Zhu, "A game-theoretic approach for communication-free voltage-VAR optimization,” 2017 IEEE Global Conference on Signal and Information Processing (GlobalSIP), Montreal, QC, pp. 1055-1059, 2017.

[122] J. Wang and N. Elia, "A control perspective for centralized and distributed convex optimization," 2011 50th IEEE Conference on Decision and Control and European Control Conference, Orlando, FL, pp. 3800-3805, 2011.

[123] M. Farivar, L. Chen, and S. Low, "Equilibrium and dynamics of local voltage control in distribution systems," 52nd IEEE Conference on Decision and Control, Florence, pp. 4329-4334, 2013.

[124] S. Bolognani, R. Carli, G. Cavraro, and S. Zampieri, "Distributed reactive power feedback control for voltage regulation and loss minimization," IEEE Transactions on Automatic Control, vol. 60, no. 4, pp. 966-981, Apr. 2015.

[125] X. Zhou, M. Farivar, Z. Liu, L. Chen, and S. Low, "Reverse and forward engineering of local voltage control in distribution networks," arXiv:1801.02015v3 [math.OC], July 18, 2018.

[126] Xue et al., "On a future for smart inverters with integrated system functions," 2018 9th IEEE International Symposium on Power Electronics for Distributed Generation Systems (PEDG), Charlotte, NC, pp. 1-8, 2018.

[127] Y. Nesterov, Introductory Lectures on Convex Optimization, 2004, vol. 87.

[128] B. He and X. Yuan, "On the $\mathrm{O}(1 / \mathrm{t})$ convergence rate of alternating direction method," SIAM Journal on Numerical Analysis, vol. 50, no. 2, pp. 700-709, 2012.

[129] B. S. He, H. Yang, and S. L. Wang, "Alternating direction method with self-adaptive penalty parameters for monotone variational inequalities," Journal of Optimization Theory and Applications, vol. 106, no. 2, pp. 337-356, Aug. 2000.

[130] S. L. Wang and L. Z. Liao, "Decomposition method with a variable parameter for a class of monotone variational inequality problems," Journal of Optimization Theory and Applications, vol. 109, no. 2, pp. 415-429, May 2001.

[131] J. Dong, M. Olama, T. Kuruganti, J. Nutaro, C. Winstead, Y. Xue, and A. Melin, "Model predictive control of building on/off HVAC systems to compensate fluctuations in solar power generation," 2018 9th IEEE International Symposium on Power Electronics for Distributed Generation Systems (PEDG) pp. 1-5, 2018.

[132] M. Gevers, "A personal view on the development of system identification," IEEE Control Systems, vol. 26, pp. 93-105, 2006.

[133] W. Favoreel, B. Moor, and P. Van Overschee, "Model-free subspace-based LQG-design," Proc. of the IEEE American Control Conference, vol. 5, pp. 3372-3376, 1999.

[134] M. Verhaegen and A. Hansson, "N2SID: Nuclear norm subspace identification," CoRR, http://arxiv.org/abs/1401.4273, 2015. 
[135] J. Qin, “An overview of subspace identification,” Computers and Chemical Engineering, pp. 1502-1513, 2006.

[136] M. Fazel, H. Hindi, and S. Boyd, "A rank minimization heuristic with application to minimum order system approximation," Proc. of the IEEE American Control Conference, pp. 4734-473, 2001.

[137] B. Telsang, S. T. Navalkar, and J. W. Van Wingerden, "Recursive nuclear norm-based subspace identification," International Federation of Automatic Control World Congress, 2017.

[138] T. Strutz, "Data fitting and uncertainty: A practical introduction to weighted least squares and beyond," Chapter 3, Springer Vieweg, 2016.

[139] J. Tovar-Pescador, "Modelling the statistical properties of solar radiation and proposal of a technique based on Boltzmann statistics," Modeling Solar Radiation at the Earth's Surface: Recent Advances. V. Badescu. pp. 55-91, Springer-Verlag, Berlin, 2008.

[140] J. Faraway and C. Chateld, "Time series forecasting with neural networks: A case study," University of Bath, Bath (United Kingdom), Research Report, pp. 95-106, 1995.

[141] P. J. Brockwell and R. A. Davis, Time series: Theory and methods, Springer Science \& Business Media, 2013.

[142] W. Ji and K. C. Chee, "Prediction of hourly solar radiation using a novel hybrid model of ARMA and TDNN," Solar Energy, vol. 85, no. 5, pp. 808-817, 2011.

[143] P. K. Jain and E. M. Lungu, "Stochastic models for sunshine duration and solar irradiation," Renewable Energy, vol. 27, no. 2, pp. 197-209, 2002.

[144] E. Ranganai and M. B. Nzuza, "A comparative study of the stochastic models and harmonically coupled stochastic models in the analysis and forecasting of solar radiation data," Journal of Energy in Southern Africa, vol. 26, no. 1, pp. 125-137, 2015.

[145] A. J. Conejo, M. A. Plazas, R. Espinola, and A. B. Molina, "Day-ahead electricity price forecasting using the wavelet transform and ARIMA models," IEEE Transactions on Power Systems, vol. 20, no. 2, pp. 1035-1042, 2005.

[146] M. Fliess and C. Join, "Model-free control,” arXiv:1305.7085, 2013.

[147] F. Lafont, J. F. Balmat, N. Pessel, and M. Fliess, "A model-free control strategy for an experimental greenhouse with an application to fault accommodation," Computers and Electronics in Agriculture, Elsevier, pp. 139-149, 2015.

[148] B. Telsang, M. Olama, J. Dong, S. Djouadi, T. Kuruganti, and Y. Xue, "Model-free control of building HVAC systems to accommodate solar photovoltaic energy," Proc. of the Power Electronics for Distributed Generation Systems (PEDG), 2018.

[149] O. Bara, M. Olama, S. Djouadi, T. Kuruganti, M. Fliess, and C. Join, "Model-free load control for high penetration of solar photovoltaic generation," Proc. of the 49th IEEE North American Power Symposium (NAPS), Morgantown, WV, Sep. 2017.

[150] H. Abouaissa, O. Hasan, C. Join, M. Fliess, and D. Defer, "Energy saving for building heating via a simple and efficient model-free control design: First steps with computer simulations," arXiv:1708.03800, 2017.

[151] D. Popo and N. Lungu, "On some differential inequalities," Seminar on Fixed Point Theory ClujNapoca, vol. 3, pp. 323-326, 2002. 
[152] B. Telsang, M. Olama, S. Djouadi, J. Dong, and T. Kuruganti, "Stability analysis of model-free control under constrained inputs for control of building HVAC systems," Proc. of the IEEE American Control Conference (ACC'19), Jul. 2019.

[153] G. Augenbroe, Y. Zhang, J. Khazaii, Y. Sun, H. Su, B. D. Lee, and J. Wu, "Implications of the uncoupling of building and HVAC simulation in the presence of parameter uncertainties," Proc. of the 13th International Conference of the International Building Performance Simulation Association, Aug. 2013.

[154] B. D. Lee, Y. Sun, G. Augenbroe, and C. J. J. Paredis, "Towards better prediction of building performance: A workbench to analyze uncertainty in building simulation," Proc. of the 13th International Conference of the International Building Performance Simulation Association, Aug. 2013.

[155] G. Augenbroe, J. Brown, Y. Heo, S. Kim, Z. Li, S. McManus, and F. Zhao "Lessons from an advanced building simulation course," Proceedings SimBuild 08, pp. 261-268, Berkeley, Aug. 2008 .

[156] Application Notes, Evvolution: Decentralized VVO Unlocks CVR Savings, Gridco Systems, 2016.

[157] OpenDSS Reference Guide, EPRI. 


\section{APPENDIX A.}

\section{A.1 BACKGROUND ON DISTRIBUTION SYSTEM VOLTAGE CONTROL}

The continuing cost reductions due to wide-scale solar deployments and innovative PV and power electronics technologies are steadily pushing PV on the path toward grid parity with other generation technologies. Despite the emergence of higher efficiency solar power technologies, such as concentrating solar power and concentrated PV, the standard standalone solar PV still asserts dominance in the solar market and is widely used in different scales of solar power systems, including residential houses, commercial buildings, and utility-scale solar farms. Utility-scale solar PV systems are currently the fastest growing market in overall capacity.

\section{A.1.1 Hardware Components of Solar PV Systems}

The needed hardware for a complete solar PV system comprises PV sources, ${ }^{1}$ power conversion, and the balance of system (BoS), which includes mounting, cabling, metering, switch board, interconnection transformer, and other mechanical and electrical accessories. More details about such systems are described as follows.

1. PV Panels or Array: Today, a PV cell can comprise several different PV materials, such as monocrystalline silicon, polycrystalline silicon, amorphous silicon, cadmium telluride, and copper indium gallium selenide/sulfide [67]. These cells are often combined to produce a PV module or panel as a more usable product. However, the power and voltage of a single PV module is still relatively low and often requires increased scaling for different solar applications and grid interconnection voltage levels. So, multiple PV modules can be connected in series to construct a PV string to generate a sufficiently high voltage and avoid a voltage-boosting power stage. To reach a higher system power level, these strings can be further connected in parallel to form a PV array, as shown in Figure A-1. For utility-scale PV systems, typical system voltage design targets of $600 \mathrm{Vdc}$ or 1,000 Vdc are used. This DC voltage is needed for power conversion and conditioning systems to deliver a 480 Vac three-phase output.

2. Power Electronics Converters: The power conversion technologies for solar PV systems have been realized by many power electronic converter topologies, including DC-DC converters and DC-AC converters, also known as inverters. These conversion technologies are necessary because the solar PV generates DC power, which is impacted by the available solar irradiance and ambient temperature. This power must be converted to AC, regulated, and conditioned to be fed to the grid. Additionally, these power electronics converters and corresponding controllers are responsible for maximizing the solar array output. ${ }^{2}$ Conventional PV inverters are designed to be current-controlled with MPPT algorithms. The solar PV exhibits nonlinear current-voltage characteristics, and numerous versions MPPT algorithms [68-69] have been created to take advantage of this feature. The perturb and observe algorithm tends to be the most common implementation. Along with maximizing the output, recent developments on solar integration standards and regulations have begun to force solar power systems to meet grid ancillary services requirements or specific grid codes. These requirements have increased the demand for smart PV inverters with new control functionalities [70]. These new inverters provide support to the grid during normal and abnormal conditions.

There are many different PV inverter topologies [71-73]. This section categorizes the inverter topologies into (1) single-phase or three-phase; (2) single power stage or multiple, usually two, power

\footnotetext{
${ }^{1}$ Actual solar cells, such as monocrystalline, polycrystalline, thin film, etc.

${ }^{2}$ Otherwise known as maximum power-point tracking.
} 
stages; (3) line-frequency, high-frequency transformer, or transformerless; and (4) two-level or multilevel topologies. These different inverter topologies demonstrate different technical and economic performances in terms of efficiency, reliability, power density, and cost.

3. BoS: The rest of the PV system hardware components (i.e., the BoS) comprise PV panel mounting racks, string combiner boxes, protection devices and switchboards, interconnection transformers, cables, and so on.

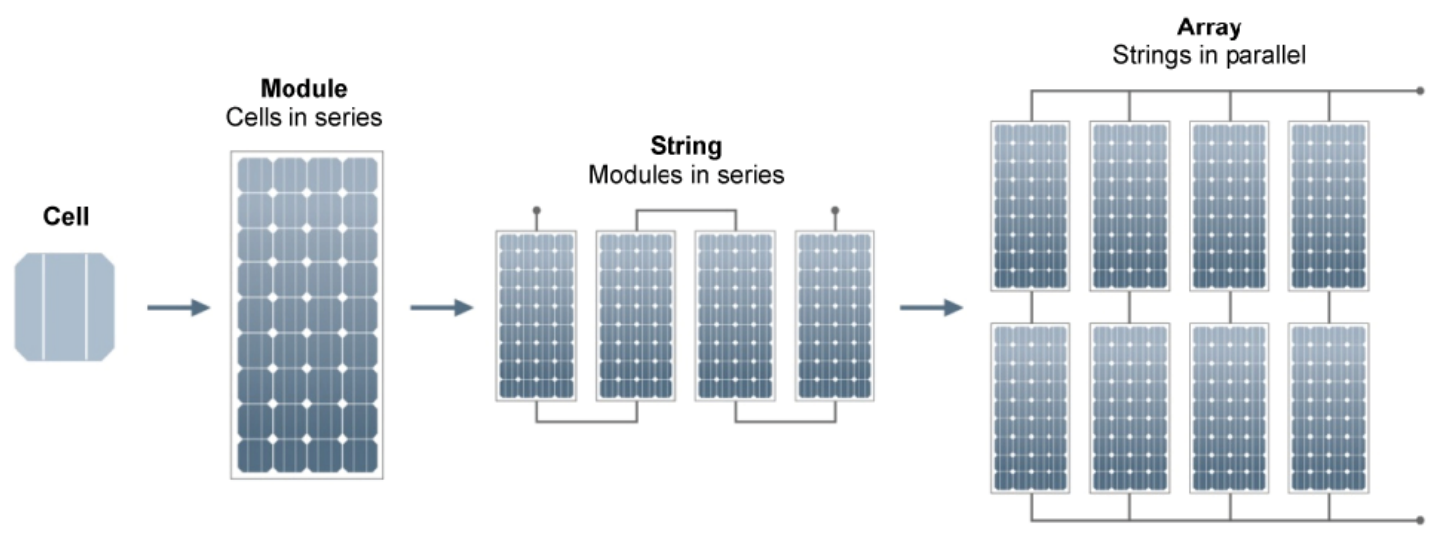

Figure A-1. Solar PV configuration from cell to array.

\section{A.1.2 Power Electronics Architectures for Solar PV Grid Interconnection}

Depending on the scale of PV installation, different power electronics architectures and configurations can be deployed for solar PV grid interconnections. The choice of centralized architecture vs. distributed architecture has implications for efficiency, reliability, and utilization. A distributed architecture generally has better solar energy utilization, whereas a centralized architecture weighs more on efficiency and reliability.

1. Centralized PV Inverter with Centralized MPPT: The centralized PV inverter with a centralized MPPT algorithm topology is shown in Figure A-2. The entire PV array is connected to an inverter either directly or through a centralized DC-DC converter, as shown. This type of configuration has the advantages of simplicity, low cost, high efficiency, and reliability. However, because of this centralized scheme, one MPPT inverter control is performed over the entire PV array, compromising the solar power utilization performance during partial shading and PV module mismatched conditions. Additionally, centralized architecture suffers from poor expandability and adaptability to customer requirements.

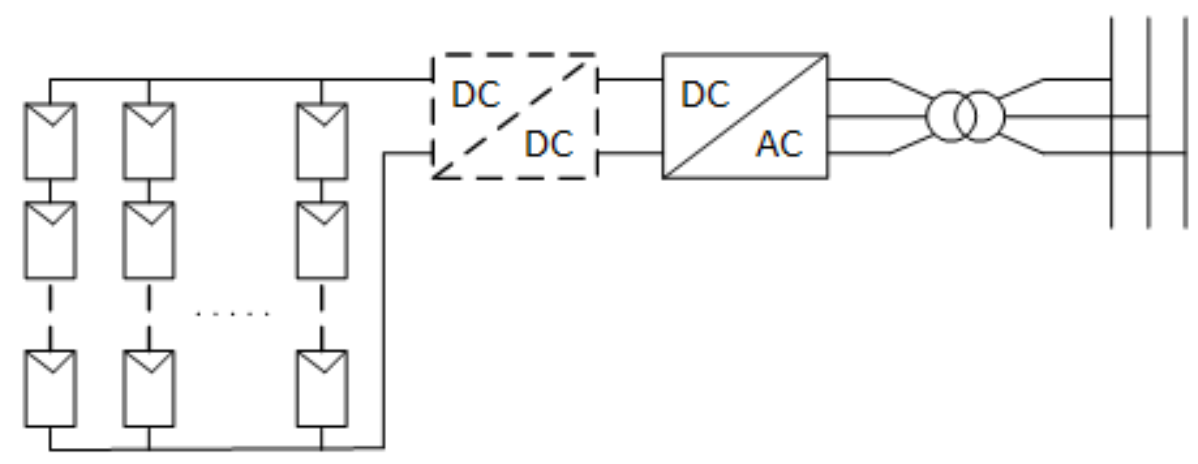

Figure A-2. Centralized PV inverter with centralized MPPT. 
Depending on the number of PV strings, a centralized inverter can comprise single-string inverters or multi-string inverters. The power rating of a single-string inverter is typically lower, and the grid interface is via a one-phase connection. This configuration was commonly observed in the early period of solar PV plant installations. Even today, large-scale solar plants mostly use configurations of multiple central inverters, also known as multi-string inverters. As an example, Figure A-3 illustrates the typical layout of a solar farm interconnected to a medium-voltage grid. The PV system capacity can be easily expanded by adding more central inverters.

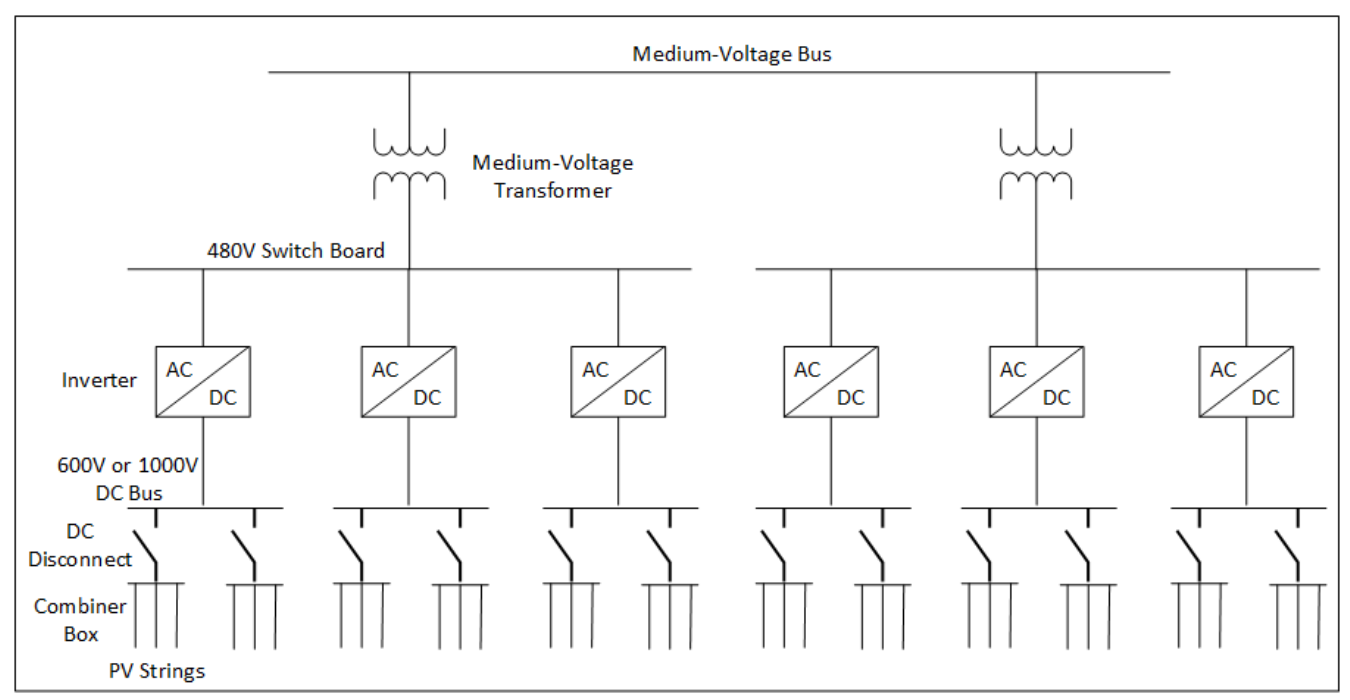

Figure A-3. PV inverter configurations in a large-scale solar farm.

2. Centralized PV Inverter with Distributed MPPT: Centralized PV inverters can also be configured with distributed MPPT architectures, which is achieved by multiple DC-DC converters to enable better solar power utilization. Four different distributed MPPT configurations are presented in Figures A-4 through A-7. In Figure A-4, each PV string has a dedicated DC-DC converter, which performs MPPT and DC voltage regulation. The PV and DC-DC converter building blocks are connected in parallel to increase the input current to the centralized inverter. A transformer is used to boost the voltage to the appropriate grid tie voltage. The utility-scale PV inverter topology is shifting toward the use of a modular cascaded structure. This type of configuration provides better harmonic spectra and facilitates lightweight filtering components [70, 74-76]. Figure A-5 shows a cascaded DC-DC converter connection of PV modules. Similar to the first topology, each PV string has a dedicated DC-DC converter. However, the PV and converter building blocks are connected in series to create a high-voltage DC link. This feature eliminates the need for a heavy and bulky linefrequency transformer for conversion to medium grid tie voltage. A cascaded H-bridge configuration is shown in Figure A-6. Similar to the first two configurations, each PV string has a dedicated DCDC converter. However, single-phase H-bridge inverters are coupled in series and in parallel configurations to boost the voltage and create the three-phase configuration for grid connection. Thus, similar to the second configuration, a line-frequency transformer is not required. The first-stage DC-DC converters with high voltage insulation can achieve the voltage boost and MPPT for the segmented PV arrays. 


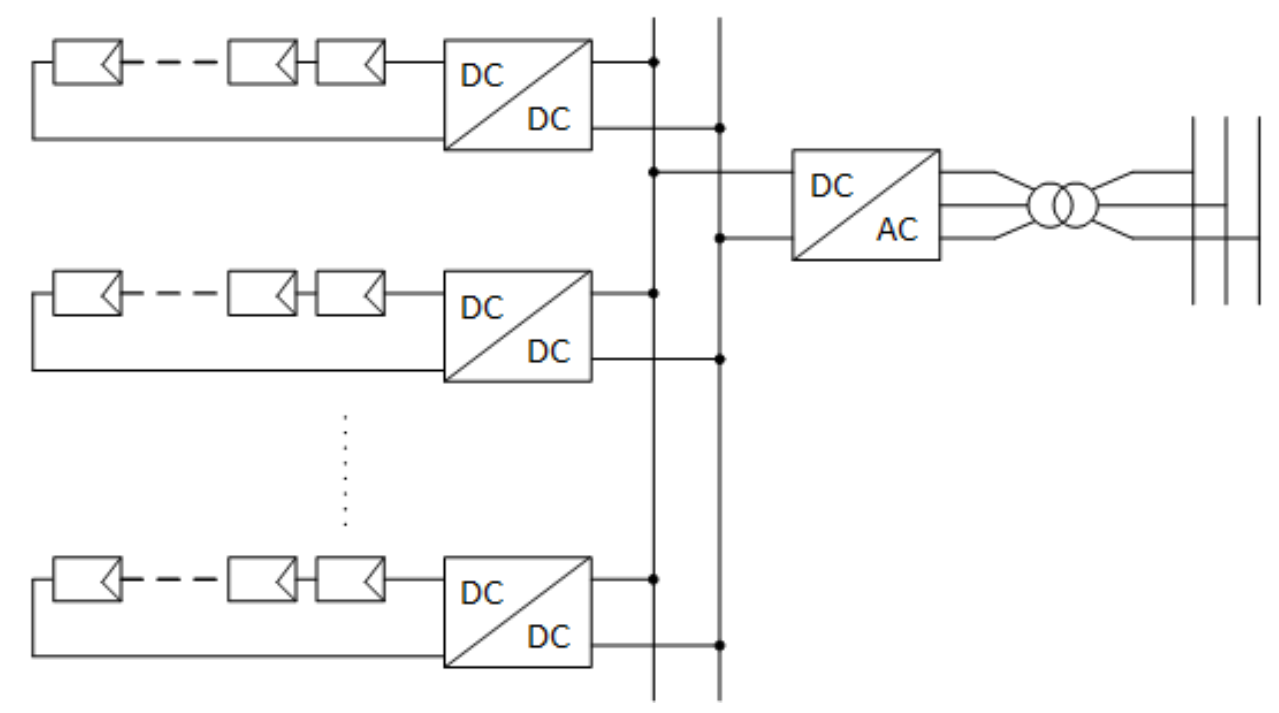

Figure A-4. Centralized inverter with distributed MPPT at string level with low-voltage DC link.

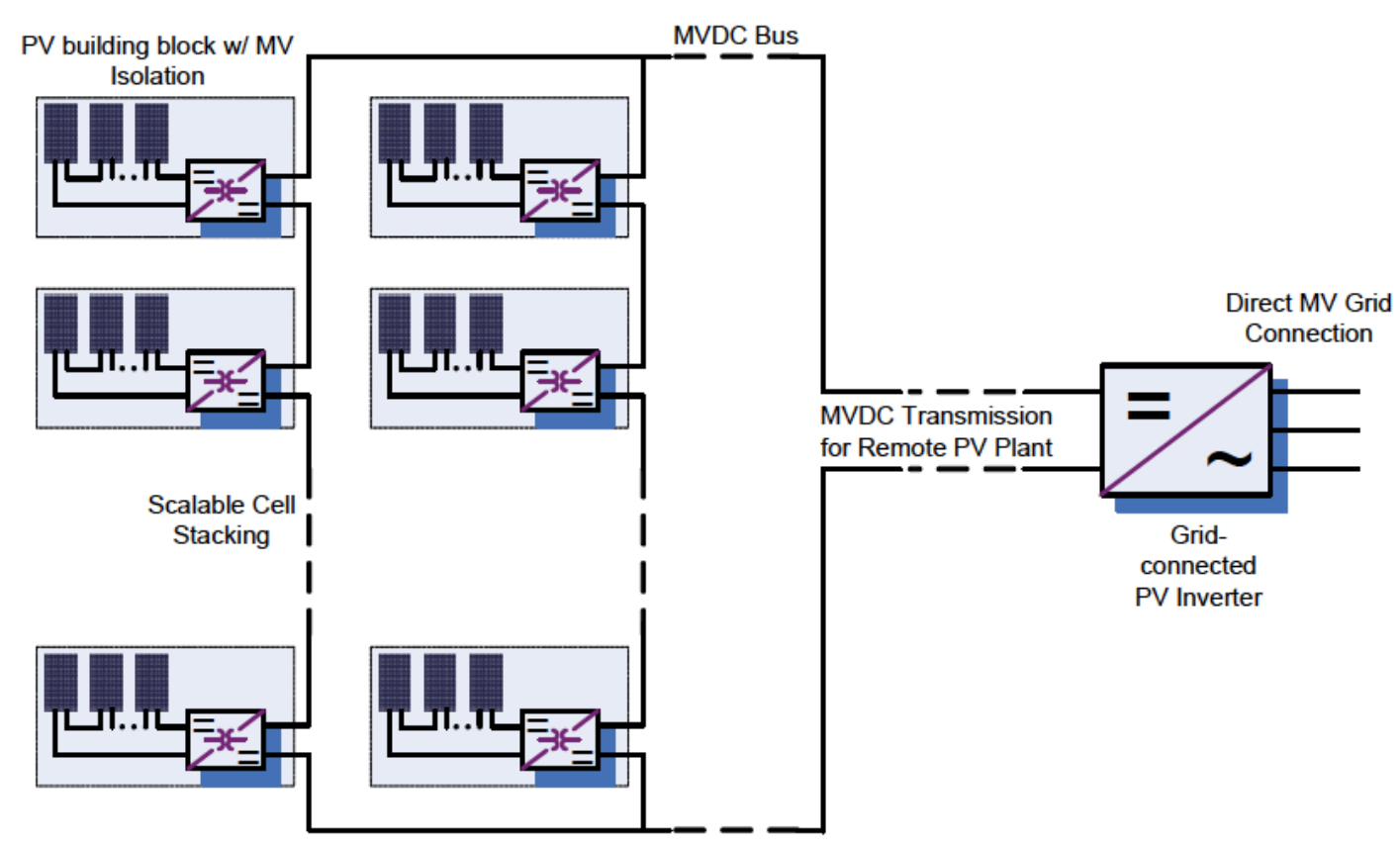

Figure A-5. Centralized inverter with medium-voltage DC transmission configuration [70]. 


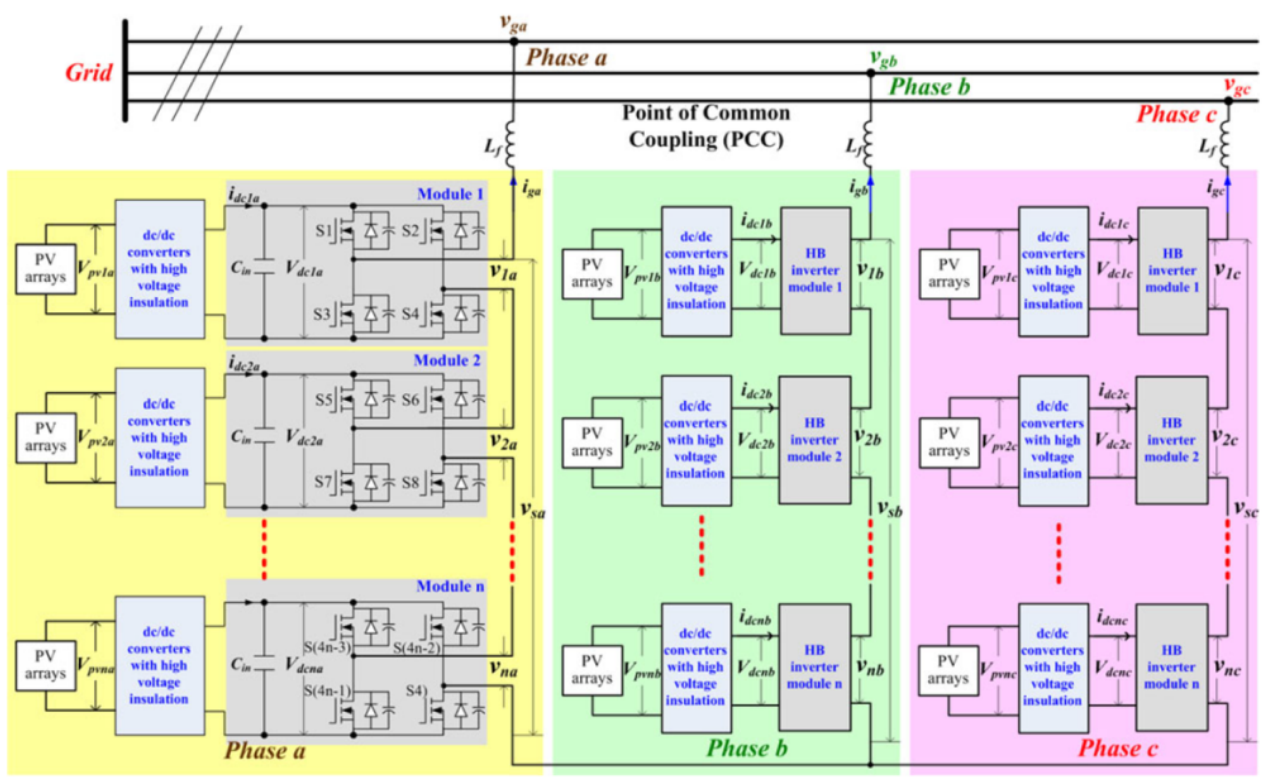

Figure A-6. Centralized PV inverter with cascaded H-bridge configuration [75].

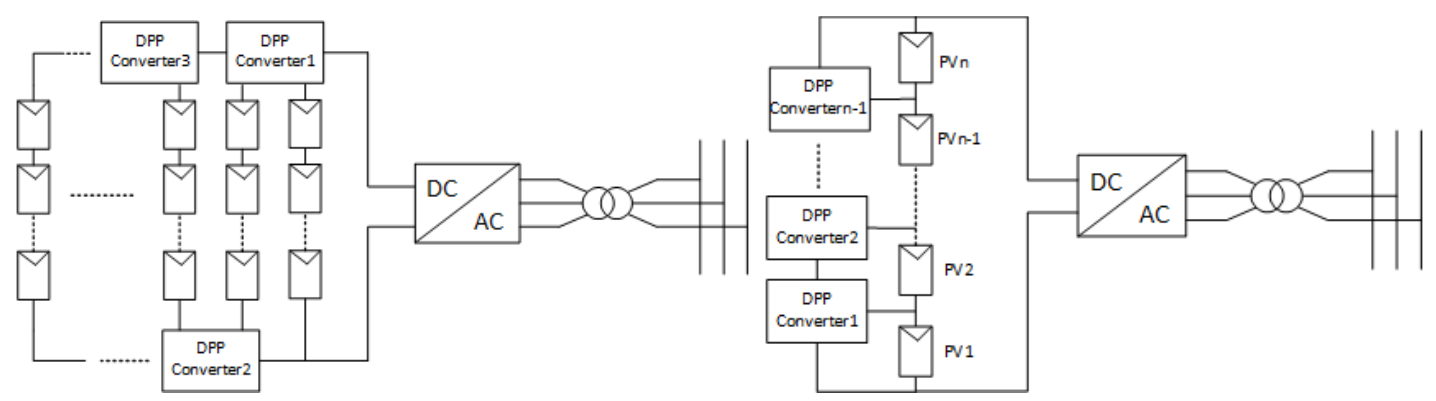

Figure A-7. Centralized PV inverter with differential power processing (DPP) configurations: voltage DPP (left) and current DPP (right).

The DPP configuration was introduced to reduce the capacity of employed DC-DC converters and hence improve the efficiency and performance of the PV system [77-78]. The system configurations for DDP are shown in Figure A-7. A basic operating challenge with PV strings is mismatch in MPP current. In current DPP configurations, the DPP converters act as controllable current sources to allow series PV panels to operate at independent current levels. Thus, every local MPP can be reached. On the other hand, for the multistring PV system, a DPP converter can be added between every adjacent pair of strings to process the differential power.

3. Micro PV Inverter ("AC PV Module”): The AC-module inverter integrates the inverter and PV module into a single electrical device [79-80]. Because the AC module inverter is attached to only one PV panel, mismatch losses between PV modules are not present and additional DC wiring is not required. This reduces the associated risk of electric arc and fire in DC wiring. This configuration is also modular, delivering a "plug and play" concept and providing an integration path for PV system enlargement [81-82].

MPPT is also performed at each PV panel, reducing the impact of cloud cover transients across a large PV system. Thus, the micro-PV inverter configuration is usually implemented in the distributed scenario, as shown in Figure A-8. The AC module inverter still has several drawbacks. First, the 
module-integrated inverter needs more complex circuit topologies to achieve high-voltage amplification.

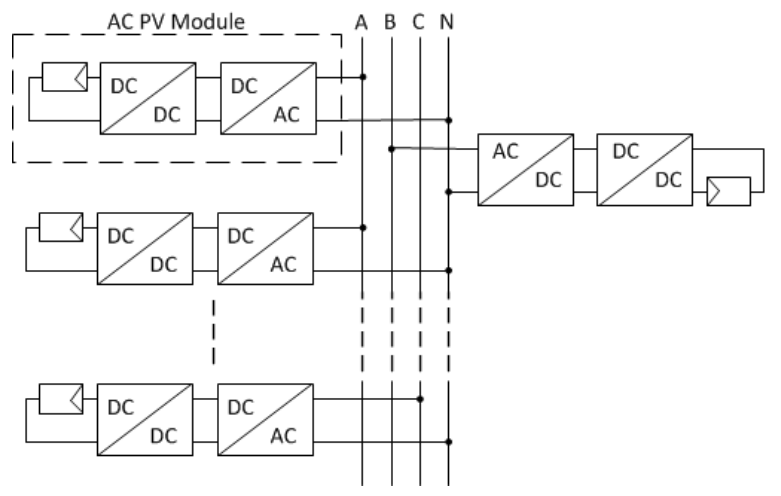

Figure A-8. Micro PV inverter.

This results in a low-converter efficiency and higher cost per watt capital investment. Additionally, the packaging of the inverter and PV module to one electrical device decreases overall system lifetime because a failure in the inverter or PV module requires the replacement of both. Today's inverter technology has an expected lifetime of about 10 years, which is far less than the lifetime of PV panels (i.e., approximately 25 years).

4. Submodule Integrated Converter: To further maximize the solar power utilization, power converters can be integrated at the submodule level, as shown in Figure A-9, with two different configurations [83-84]. Individual MPPT can be achieved for different groups of solar cells within one solar PV panel so that the global MPP can be tracked, even when the panel is partially shaded.

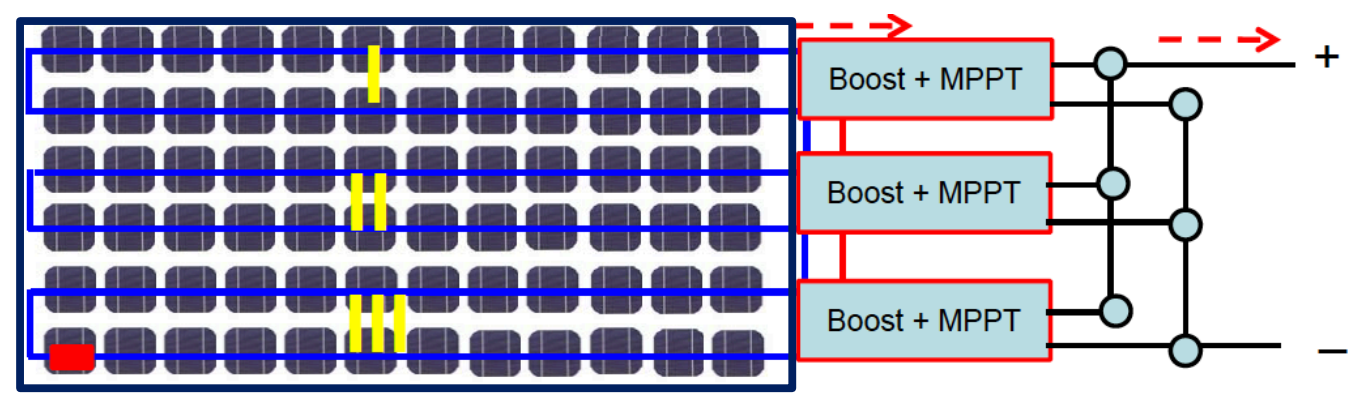

(a) Parallel connection.

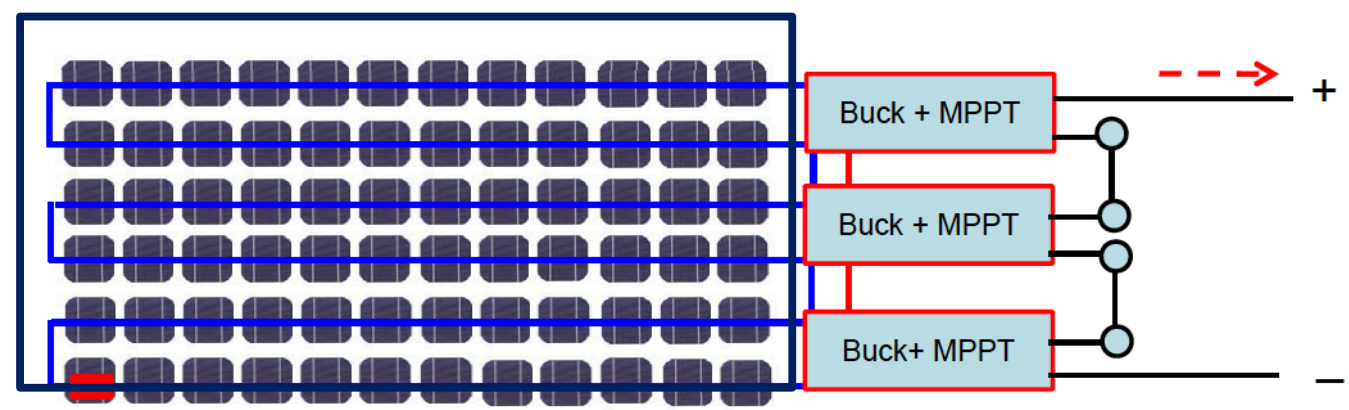

(b) Series connection.

Figure A-9. Submodule integrated converters. 


\section{A.1.3 Distribution System Voltage Control}

Electric distribution systems refer to the portions of medium- and low-voltage electric power grids. In North America, standard medium voltages range from $600 \mathrm{~V}$ to $69 \mathrm{kV}$, and low voltages are any voltage below $600 \mathrm{~V}$. Typically, residential houses are connected to low-voltage lines, $120 \mathrm{~V}$ single-phase or $240 \mathrm{~V}$ split-phase; commercial buildings are connected to three-phase 480 or $690 \mathrm{~V}$. These low-voltage loads are called secondary customers from the utility perspective. The lower end of medium-voltage distribution grids serves the primary customers at $4.16-13.8 \mathrm{kV}$, and the higher end of medium-voltage distribution grids serves subtransmission customers at $26-69 \mathrm{kV}$.

Different distribution system architectures are adopted in different areas and different countries. The choice of distribution architectures has cost and reliability concerns [85]. The subtransmission and primary distribution systems can generally be designed in looped, radial, meshed (also called interconnected or spot network), or T-type structures. In the United States, two primary distribution system architectures are commonly used [86]. The radial distribution structure is typically used in rural areas, whereas the meshed network structure is more deployed in densely populated city areas. The radial distribution feeder circuit, an example of which is shown in Figure A-10, traditionally has only one source from the distribution substation and no switch device linking that circuit with another, thus suffering from reliability concerns if a fault occurs near the substation and power quality issues at the feeder end due to the voltage drop or power loss of the long feeder line. This structure has a historic root and it remains the majority in the country. In high-load city areas, meshed secondary networks [87], as shown in Figure A-11, are installed, which comprise multiple distribution voltage-level feeders, each serving several underground transformers installed in vaults; the low-voltage secondaries of the transformers are interconnected, and customers are served from these interconnected secondaries. This type of architecture provides a very high degree of service reliability to the customers; several components can be out of service at any one time without causing a loss of service. However, higher construction cost has limited its expansion.

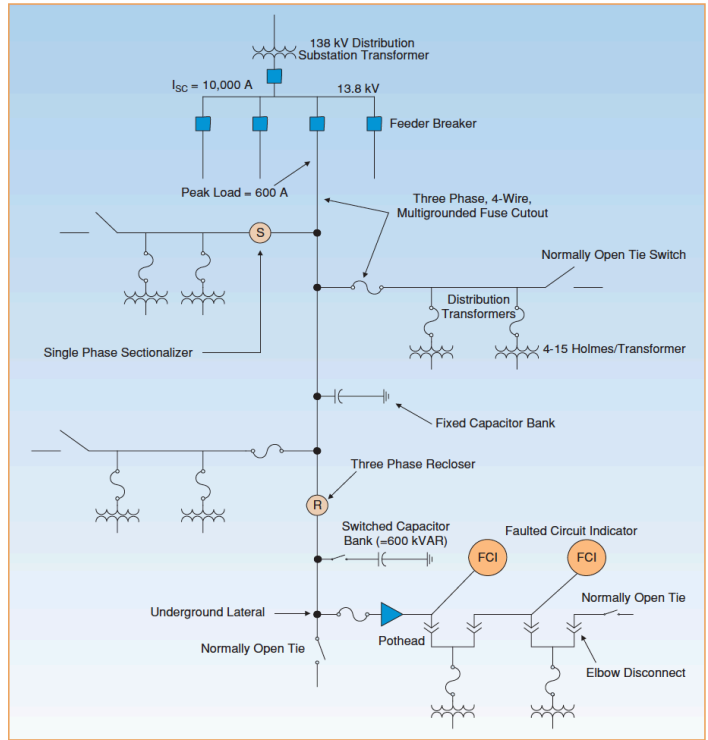

Figure A-10. Typical radial distribution system [86].

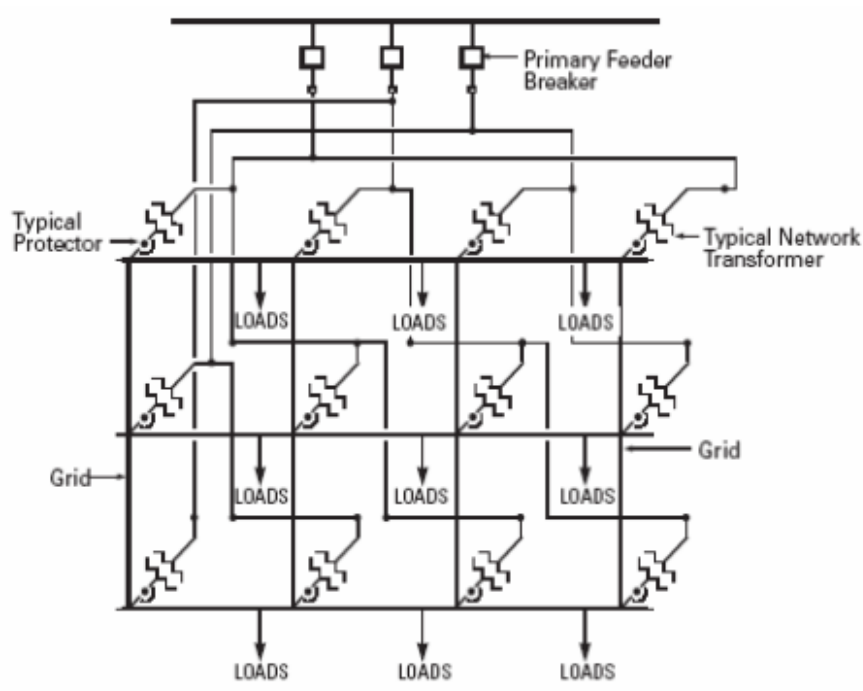

Figure A-11. Secondary grid network distribution system [87]. 
Recent extreme natural events, such as superstorm Sandy, have called for grid resilience, which requires distribution systems capable of fast restoration and self-healing. The technology advances of distributed intelligence and distribution automation [88] enable these capabilities, which can change the radial distribution structure dynamically to improve reliability and resilience by using devices such as sectionalizers, reclosers, power flow controllers, and fault location/isolation/service restoration or fault detection/isolation/restoration.

Distribution utilities are obligated to guarantee power quality to customers. The term power quality is associated with the electromagnetic phenomena, such as: (1) transients, (2) short-duration variations, (3) long-duration variations, (4) voltage unbalance, (5) waveform distortion, (6) voltage fluctuations, and (7) power frequency variations. The voltage control devices are primarily deployed to handle type 3 disturbances, particularly preventing undervoltage and overvoltage via voltage regulation at fundamental frequency. This problem is also referred to as the nodal voltage profile or maintaining viable voltage level and is investigated below. Another type of voltage stability problem is voltage collapse, which investigates the overloading point (i.e., bifurcation) at which the voltage will collapse.

To improve nodal voltage profiles in medium-voltage distribution networks, different voltage control devices are used by distribution utilities. Substation transformers are typically equipped with on-load tap changers (OLTCs). Step voltage regulators (SVRs) can also be installed in the middle of the feeder to boost the voltage by a transformer with LTCs. Voltage drops can also be caused by the loads that absorb large reactive power (i.e., lagging PF). Reactive power compensation devices, such as fixed or adjustable capacitor banks - and various power electronics-based compensators, such as static VAR compensators (SVCs) and static synchronous compensators (STATCOMs) - are installed in the substation and along the feeders or close to particular loads. In low-voltage distribution networks, direct voltage-control devices are seldom present traditionally, as illustrated in Figure A-12. In recent years, smart inverters and various grid edge technologies have evolved in the low-voltage distribution system to provide grid-supporting ancillary functions. However, these new voltage-control devices present impacts on the operation of conventional voltage-control devices, and they must coordinate to maintain proper voltage profiles and reduce system loss.

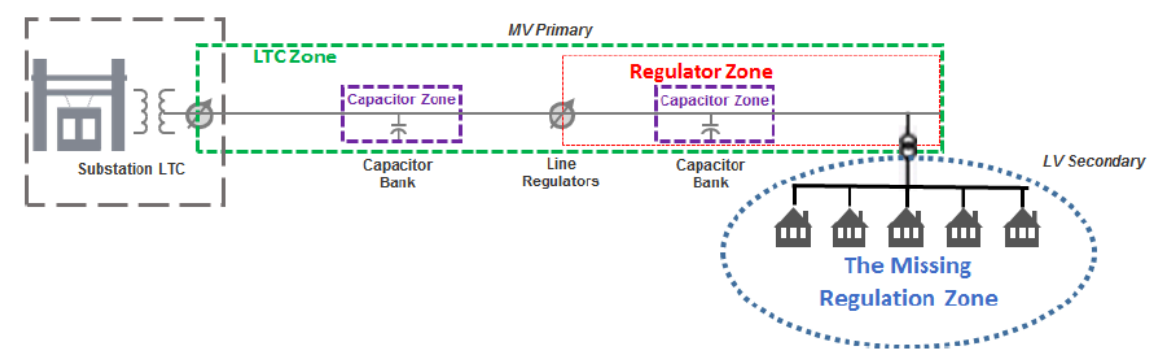

Figure A-12. Distribution system voltage regulation zones [156].

The voltage regulation problem is traditionally approached with a hierarchical structure: offline optimization and online control, as shown in Figure A-13. The offline optimization problem defines the optimal settings for the online voltage control reference and the optimal sequences of connection and disconnection of the capacitors. This process is typically performed 1 day ahead based on $24 \mathrm{~h}$ load forecasting. The online control problem targets the control of OLTCs via closed-loop regulation to maintain the voltage amplitude at the reference value. The controlled voltage can be the transformer secondary voltage or the estimated voltage at the feeder end by the line drop compensator. 


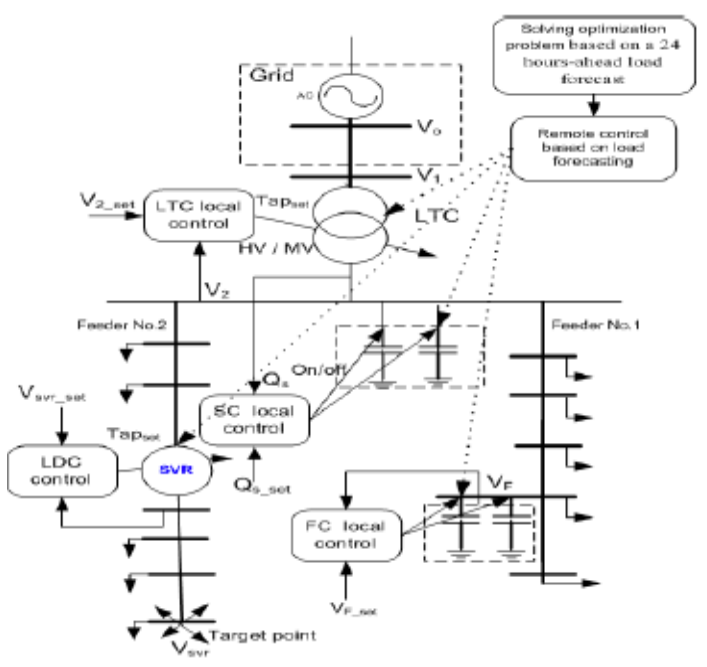

Figure A-13. Conventional voltage and reactive power control in distribution systems [93].

The LTC is placed on the substation transformer and operates on a three-phase basis, whereas SVRs regulate individual phases and can be placed flexibly on any feeder location where required (e.g., at feeder bus or head or in the middle of a long feeder line). A voltage regulator comprises an autotransformer, LTC, and voltage regulator control. A voltage change is obtained by changing the series winding taps of the autotransformer. The position of the tap is determined by a line drop compensator. Standard step regulators contain a reversing switch, enabling a $\pm 10 \%$ regulator range for type A SVRs and $+10 \%$ and $-8.3 \%$ for type B SVRs, usually in 33 steps. This amounts to a $5 / 8 \%$ change per step, or $0.75 \mathrm{~V}$ change per step, on a $120 \mathrm{~V}$ base. The SVR control circuit requires the following settings.

- Voltage level: Desired voltage to be held at the load center, typically between 114 and $126 \mathrm{~V}$.

- Voltage bandwidth: The allowed variance of the load center voltage from the set voltage level.

- Time delay: The length of time that a higher or lower operation is called for before the actual execution of the command. This setting is intentional to avoid unnecessary accelerated wear and tear of the tap changer for short-duration voltage excursion outside the bandwidth caused by events related to motor starting. It is typically set in the range of 30 to $90 \mathrm{~s}$.

- Line drop compensator: Set to compensate for the voltage drop between the regulator and the load center. The settings consist of $\mathrm{R}$ and $\mathrm{X}$ settings in volts corresponding to the equivalent impedance between the regulator and load center when the line is carrying the current transformer's rated primary current.

Smart inverters can provide autonomous Volt/VAR control or PF control [89] to mitigate local voltage fluctuations and voltage rise issues due to solar power injection at the point of interconnection. However, a market incentive mechanism has yet to be established for nonutility-supplied reactive power.

Additionally, the provision of reactive power capabilities normally requires excess capacity on the inverter and thus oversizing hardware design or active power production curtailing. For smart inverters that provide additional voltage support, communications and standardized control functions are required by the IEEE standards and specific grid codes, which are reviewed in the next section, to ensure that these functions are coordinated with distribution system regular voltage control operations and are enabled only when appropriated to do so [90]. Figure A-14 shows a control architecture and configuration [91] for the OLTC controller to consider the voltage at the feeder end by either estimate or remote monitoring. 


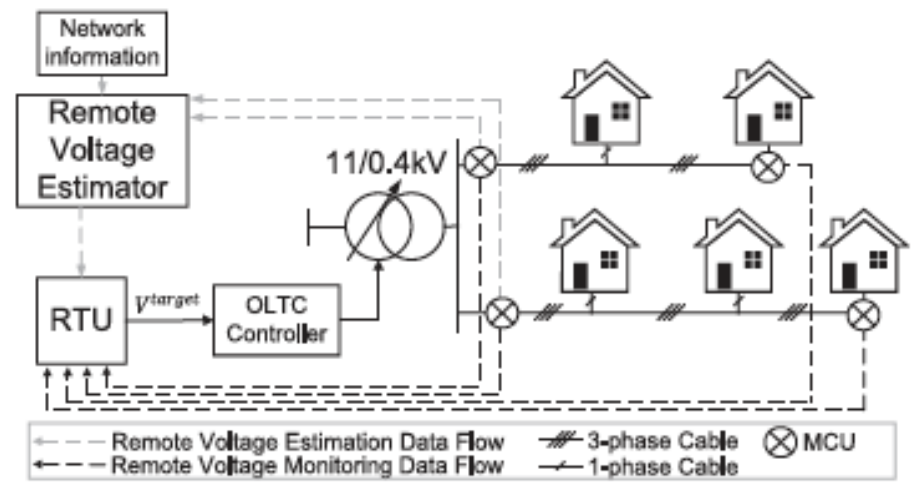

Figure A-14. Control architecture with and without remote monitoring [91].

The voltage control technologies in distribution systems are continually evolving. In the 1970s and early 2000 s, with the energy and electricity crisis concerns, utilities looked at conservation voltage reduction (CVR) to increase distribution system efficiency and conserve energy. Over the years of technology improvement and communication establishment at most distribution equipment, the focus of minimizing energy consumption shifted to controlling the voltage and volt-ampere-reactive power levels (i.e., VAR) in near-real time. This is known as voltage VAR optimization (VVO). There are two different VVO control structures: centralized and decentralized. Centralized VVO approaches use LTC transformers, voltage regulators, and fixed/switched capacitor banks to control voltage and reactive power flow. There are three different centralized VVO approaches [92]: supervisory control and data acquisition (SCADA) rule-based VVO, DMS model-driven VVO, and hybrid VVO.

The rule-based system shown in Figure A-15 uses a VVO processor in conjunction with a remote terminal unit to control the automated devices. The design can incorporate the voltage of the feeder end and change the tap position or capacitor to on or off based on a set of rules, programmed voltage, and VAR thresholds to achieve minimized feeder losses, minimized energy consumption, minimized power demand, or any combination of these. The communications between the VVO processor and line devices can be via SCADA or cellar phone.

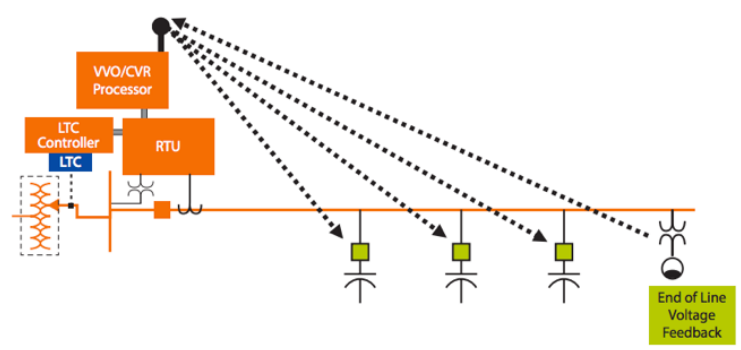

Figure A-15. The SCADA rule-based VVO approach

[94].

The model-driven VVO approach shown in Figure A-16 takes advantage of the most information and tools available in a DMS - including near-real-time network configuration, advanced metering infrastructure, outage management system, geographic information system (GIS), and customer information system - to develop and execute an optimal switching plan for all the controllable devices to reach a desired result. The core engine is the IVVC, which runs an online power flow analysis and 
optimization to create a VVO switching plan. The hybrid VVO approach emerged more recently, using autoadaptive controllers in the field devices or at the system level to respond to changing conditions. These autoadaptive controllers are based on either closed-loop control with real-time voltage measurement or historical behavioral patterns to optimize the power flow based on the rules provided by the utility. Additionally, decentralized or agent-based VVO schemes [95-96] have attracted attention to tackle the control scalability. More detailed reviews will be conducted in next quarter.

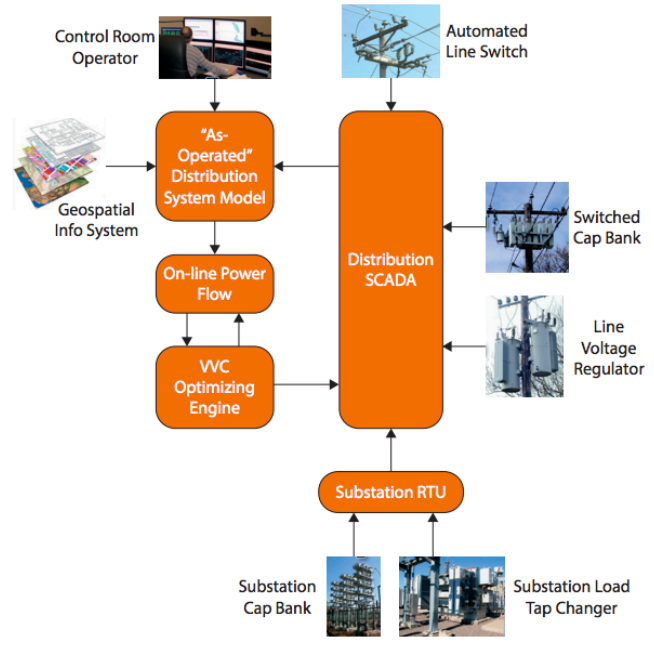

Figure A-16. The DMS model-driven IVVC scheme [94].

Distribution voltage control is usually associated with three objectives: power quality, energy efficiency (i.e., reducing power loss and/or peak load), and voltage stability, also known as voltage instability or collapse. In this report, a fourth-dimension objective is added, extending the lifetime of voltage regulation devices, such as SVRs and distribution transformers with OLTCs.

Various control architectures for the distribution nodal voltage control to ensure power quality to end customers were reviewed previously in this section. The term power quality refers to a wide variety of electromagnetic phenomena that characterize the voltage and current at a given time and at a given location in the power system. Among those disturbances, the nodal voltage controls mainly target preventing undervoltages and overvoltages via voltage regulation at fundamental frequency [97]. For harmonic-related power quality issues, various passive or active power filters (APFs) can be installed to compensate voltage harmonic distortions due to nearby nonlinear loads. Within the APF family, the unified power quality conditioner (UPQC), which integrates shunt compensation and series compensation in one system, is employed in a power distribution system, whereas the unified power flow controller (UPFC) is employed in a power transmission system. Further discussion on the details of UPQCs is beyond the scope of this report. However, a comprehensive overview of different UPQC power electronics converter topologies and system configurations can be found in the literature [98-99].

Traditionally, electrical utility companies have owned the voltage regulation equipment, such as distribution transformers with OLTCs, SVRs, and fixed or switching capacitor banks, which are installed only in the medium-voltage distribution grid. Centralized control schemes are typically adopted in practice by the utility company. In recent years with the emergence of residential PV systems (a few kilowatts), commercial building solar PV systems (a few hundred kilowatts), and utility-scale megawatt solar farms, there has been demand for various grid services provided by solar PV inverters in addition to their primary energy feeding functions. Thus, new voltage control devices are present in both medium-voltage distribution zones (typically a MW-scale solar farm connected to a $4.16-69 \mathrm{kV}$ grid) and 
low-voltage distribution zones (residential PV at single-phase $120 \mathrm{~V} / 240 \mathrm{~V}$ and commercial building PV at three-phase $480 \mathrm{~V}$ ). Usually, the voltage control problem and reactive power control problem [100] are highly coupled with reactive power compensation to improve the load PF and in the meantime support the voltage.

As forecasted in the literature [70,101-102], solar PV inverters are being staged into smart inverters. Relevant IEEE standard 1547(2018) requires smart control functionalities provided by inverter-based DERs, such as solar PV and energy storage systems. The DER is required to provide voltage regulation capability by changes of active power, and this active participation in voltage regulation shall be approved by the area electric power system (i.e., the distribution utility operator). Specifically, the DER shall be capable of activating any of the following reactive power control modes individually [103]:

- $\quad$ adjustable constant PF mode,

- voltage-reactive power (volt-VAR) mode,

- active power-reactive power mode (watt-VAR), and

- adjustable constant reactive power mode.

In addition to reactive power control, the DER is also required to provide a programmable voltage regulation capability by changes of active power [103] (voltage-real power [volt-watt] mode).

Because of the emerging voltage control devices on the customer side and their distributed nature, research interest in decentralized voltage and reactive power control philosophy [93] has naturally spiked. However, the operation impact of new voltage control devices must be studied first.

The effects of voltage control by the DER and the interactions between the DER and the utility voltage regulation devices (e.g., capacitor banks, voltage regulators, and LTCs) were investigated [90, 104-105] on computer simulation bases. Without proper coordination by distribution network planners and operators, the voltage control provided by the DER might introduce adverse effects, including control interactions, operational conflicts, steady-state voltage variations, and oscillations. In the paper by Ranamuka et al. [90], a sample medium-voltage distribution feeder system, shown in Figure A-17, was simulated and analyzed with two feeder circuit configurations and four case studies. It was observed that more tap operations are required in SVRs to correct the voltage according to their voltage reference value. This is because the DG voltage control mode operation and associated voltage reference values of the DG units tend to damp the voltage correction, which could lead to unnecessary capacitor bank switching operations and exhausted tap operations. Also, the tap operations of voltage regulators do not significantly affect the remote end bus voltage, mainly due to the fast voltage control action of the inverter-based DG. The total number of conflicting operations in voltage regulators and capacitor banks are also reported in the paper, as replicated in Table A-1, based on 1 year 10 min time resolution simulations. These simulation results highlight the DG and voltage-regulating device interactions and their possible adverse effects under different system operational conditions. 


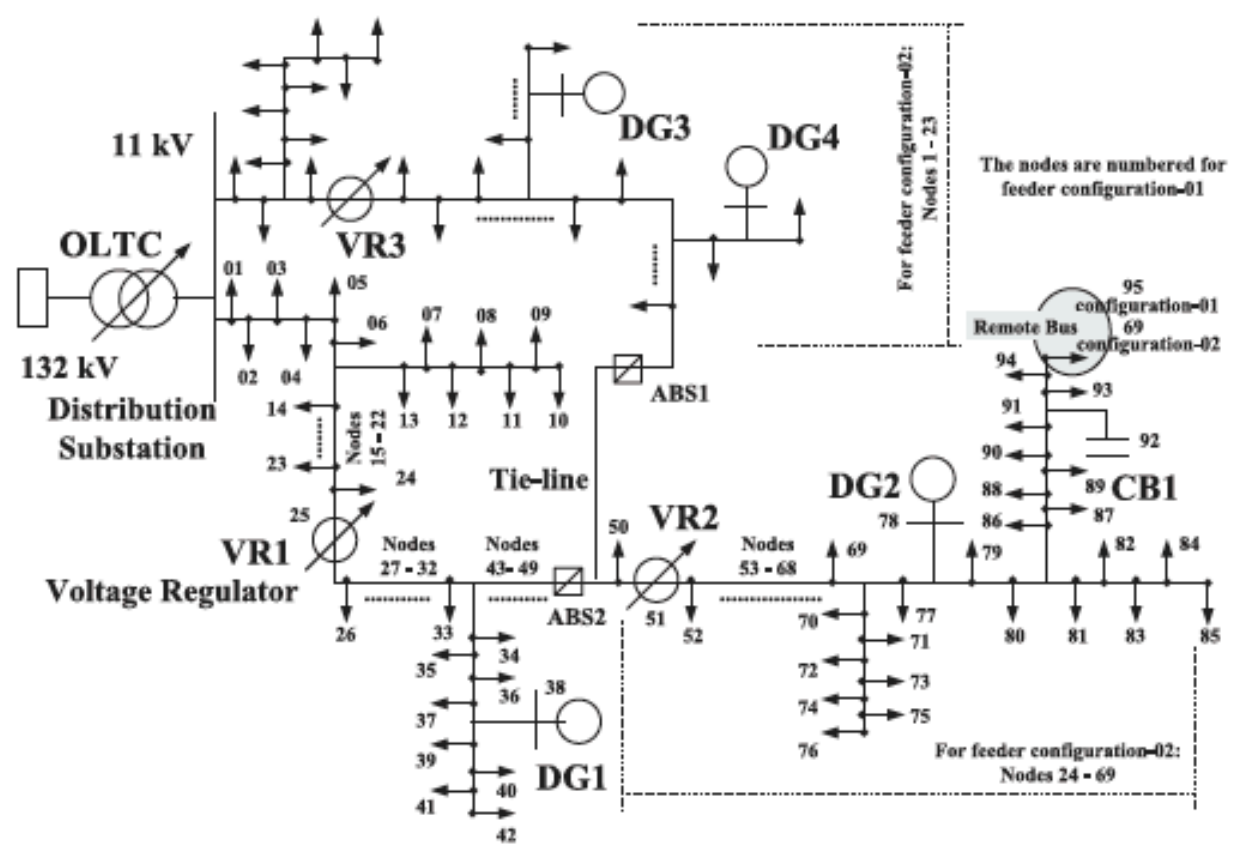

* Feeder Configuration - 01: System with ABS-1 opened and ABS-2 closed (OLTC, 2 SVRs, DG1, DG2 and CB are in operation)

* Feeder Configuration - 02: System with ABS-1 closed and ABS-2 opened (OLTC, 2 SVRs, DG2 and CB are in operation and DG3, DG4 are disconnected)

Figure A-17. Topology of a realistic MV distribution feeder system in New South Wales, Australia [90].

Table A-1. Number of conflicting operations associated with DG voltage-regulating device interactions, as found in Ranamuka et al. [90]

\begin{tabular}{|c|c|c|c|}
\hline Simulated Control States & $\begin{array}{l}\text { No. of Conflicting Operations in VRs and } \\
\text { CBs due to Simultaneous Responses }\end{array}$ & $\begin{array}{l}\text { No. of Conflicting Operations in VRs and } \\
\text { CBs due to Nonsimultaneous Responses }\end{array}$ & Remarks \\
\hline $\begin{array}{l}\text { Case Study-1 (Case-1) } \\
\text { Data: Table-I } \\
\text { Simulation: Fig. } 10\end{array}$ & VR Tap Operations = 17520 (per year) & VR Tap Operations = 35040 (per year) & $\begin{array}{l}* \text { Additional VR, CB operations } \\
\text { due to interactions }\end{array}$ \\
\hline $\begin{array}{l}\text { Case Study-1 (Case-2): } \\
\text { Data: Table-I } \\
\text { Simulation: Fig. } 11\end{array}$ & N/A & $\begin{array}{l}\text { CB1 Switching }=17520 \text { (per year) } \\
\text { VR Tap Operations }=26280 \text { (per year) }\end{array}$ & $\begin{array}{l}* \text { Additional VR, CB operations } \\
\text { due to interactions }\end{array}$ \\
\hline $\begin{array}{l}\text { Case Study-2 (Case-3): } \\
\text { Data: Tables-I, II } \\
\text { Simulation: Fig. } 12\end{array}$ & VR Tap Operations = 52560 (per year $)$ & VR Tap Operations = 43800 (per year) & $\begin{array}{l}* \text { Additional VR, CB operations \& } \\
* \text { Voltage rise, due to interactions }\end{array}$ \\
\hline $\begin{array}{l}\text { Case Study-3 (Case-4): } \\
\text { Data: Table-I, III } \\
\text { Simulation: Fig. } 13\end{array}$ & N/A & $\begin{array}{l}\text { CB1 Switching }=17520 \text { (per year) } \\
\text { VR Tap Operations }=105120 \text { (per year) }\end{array}$ & $\begin{array}{l}* \text { Additional VR, CB operations \& } \\
* \text { Voltage drop, due to interactions }\end{array}$ \\
\hline
\end{tabular}

A coordinated voltage control strategy or volt-VAR optimization thus becomes vital to maintain the desired voltage at any point along the feeder for modern distribution system operations. This is typically achieved centrally by using a distribution automation system (e.g., SCADA system) or advanced DMS (ADMS). VVO, as a key function of ADMS, is designed to optimize the voltage and reactive power flow in the distribution network by using all the possible control devices. The objective function is typically to minimize the system power loss, energy consumption, or a combination of both, and the constraints are the maximum current values in feeder lines or transformers and keeping all bus voltages within a range. Besides the traditional standalone volt-VAR controllers and rule-based VVO, the IVVC is the most advanced VVO approach. The IVVC, also known as the DMS model-based VVO approach, uses real-time 
measurements, a distribution system model, and an online power flow computation function, as well as an optimization engine to calculate the optimal set of control actions for all control equipment and then send the control set points to the local controllers embedded in each piece of control equipment via SCADA or a communication link.

Optimization algorithms include analytical methods, numerical methods, heuristic methods, and AI methods. Numerical VVO methods use mixed-integer linear programming, mixed-integer quadratic constrained programming, sequential convex programming, MPC, dynamic programming, and ordinal optimization techniques. Most heuristic methods found in the literature are based on evolutionary optimization algorithms, including genetic algorithms, particle swarm optimization, the teaching-learning algorithm, simulated annealing, ant colony optimization, tabu search, the shuffled frog leaping algorithm, memetic algorithms, honeybee mating optimization, the bacterial foraging optimization algorithm, bee swarm optimization, the gravitational search algorithm, and so on. The AI methods include neural networks, adaptive neuro-fuzzy inference, and multiagent systems. A detailed review of various VVO methods can be found in Satsangi and Kumbhar [106].

Flexible AC transmission systems (FACTS) have been deployed in the transmission system for voltage control and reactive power compensation, such as SVCs using a thyristor-controlled reactor (TCR), or thyristor-switched capacitors and UPFCs. There is a trend to promote similar control functions using newer self-commutated semiconductor switching devices at the distribution level to create distribution static synchronous compensators (D-STATCOM) and UPQC (as discussed previously) and to address voltage control and other power quality issues. More detail on D-STATCOM research efforts are found in the literature [96, 107-109]. Furthermore, there is a bold vision to achieve a flat voltage profile across the entire distribution feeder circuit without communications. One concept is to add more capacitors along the feeder and allow the capacitor banks to be the primary voltage-regulating devices while the LTCs of voltage regulators or distribution transformers address only emergency or dramatic voltage changes.

Buildings consume two-thirds of the electricity produced in the United States, and a significant portion of the building load is dispatchable, making them responsive to electrical grid needs as an effective demand-side management tool. Direct load control has been adopted by the utilities to manage peak time demand. More recently, various demand-response programs were implemented to encourage load curtailment by the time of use with financial incentives. However, these methods would not solve the voltage problem investigated in this report at the minute timescale level. In this project, the team used the building HVAC loads with model-predictive and adaptive controls to mitigate the dynamic voltage issues when the solar generation mismatches the load demand. As mentioned previously, reducing excessive voltage regulation operations and extending the lifetime of existing mechanical-based voltage control equipment (e.g., LTCs) can be a control objective. This is a feasible approach from the load control perspective because it does not require significant additional capital investment. In this project, the team developed adaptive control strategies for building HVAC loads to mitigate the impact of solar power minute-to-minute fluctuations in the LTCs of distribution transformers and SVRs. Because single HVAC load has a relatively slow response, a fleet approach was adopted to seek an aggregation effect and diversified dynamics up to 5-10 minutes of control resolution. This work demonstrates a technical leap over conventional demand response programs and has a market potential to increase the hosting capacity of solar power in a distribution feeder.

In summary, several new voltage control devices and approaches in distribution systems were reviewedincluding smart inverters, coordinated VVO, distribution STATCOM, and adaptive building HVAC load controls - that represent ongoing trends in research and practical applications to transition to an active distribution system. 


\section{A.2 COORDINATION OF BUILDING HVAC LOAD CONTROL WITH SMART PV INVERTERS}

Individual building HVAC control has relatively slow dynamics in the range of tens of minutes (e.g., $10 \mathrm{~min}$ ). In this project, the team aggregated and coordinated a fleet of available HVAC units (e.g., 100 units) to enhance the resolution of load control dynamics and control performance. However, under certain extreme scenarios, it is possible that significant portion of the coordinated $100 \mathrm{HVAC}$ units will get into lock-off status during some highly dynamic solar production hours. Therefore, additional control resources are sought to manage the fast voltage fluctuations due to fast solar power variations. The encouraging factor is that the fast- or high-frequency components in solar power spectrum are relatively small and do not occur frequently based on the solar spectral analysis presented in prior reports. This section investigates different coordination and integration schemes of building HVAC load control with smart solar PV inverters and develops a new distributed voltage controller (DVC) to fill the gap in existing work from the literature survey.

\section{A.2.1 Control Integration at ADMS Level}

One common practice for DSOs is to adopt a centralized optimization and control structure [110]. At the distribution system level, all the voltage control devices - including distribution substation transformer with LTCs, feeder voltage regulator, capacitor banks, D-STATCOM, and smart inverters-must be coordinated by the IVVC within the ADMS for optimal operations to maintain the desired voltage at any point along the feeder.

The dynamic load controls that the team is currently developing can be integrated into this optimization engine at the distribution system level, which uses real-time measurements, a distribution system model, and an online power flow computation function to calculate the optimal set of control actions and then sends the control set points to the local load controllers via SCADA or a communication link. However, the dynamic load controls can also operate in the background independent of the DMS model-based VVO to track and offset the solar power minute-to-minute fluctuations, which in turn reduce the operation workload of LTCs.

\section{A.2.2 Control Coordination at Local Node}

Emerging distribution voltage management approaches are moving toward decentralized or completely distributed structure [33]. For the sake of scalability and plug-and-play, the team adopted a distributed control approach without increasing the DER management system (DERMS) control burden. Specifically, at the local area or node level, the developed load controller will communicate with the solar PV inverter onsite to obtain the real-time solar active power data. Typically, the voltage control of smart inverters is based on fixed or dynamic reactive power compensation (absorbing and injecting) by using local voltage measurement, whereas the dynamic load control is based on direct real power changes. So, the two control techniques complement each other in the sense that the load controller controls real power in the background - indirectly controlling the voltage - while the PV inverter or any other smart inverter (e.g., energy storage) controls reactive power to regulate the voltage precisely and for a shorter period at a timescale of seconds. There are economic benefits associated with this integration because with load controls, system cost can be reduced without the need of additional energy storage and oversizing of the smart inverters to provide additional reactive power, and the renewable energy use can be increased without potential solar power curtailment in smart inverter-only configurations. Like any other control technique, dynamic load control also has limited dynamic capacity, depending on the temperature, occupancy and satisfaction, and aggregate quantity. So, the smart inverter and dynamic load control can be integrated more closely through communications. A system controller on top of a smart inverter and load controller will be designed to optimally determine the sharing of voltage control responsibility based 
on real-time measurements (e.g., temperature, solar power) in terms of real-time control capacity, economics, and human factors.

\section{A.2.3 The Need for Distributed Control Approach}

With the continuous increasing number of DERs, the DMS and operator might become incapacitated due to complex optimization problem size and/or limited control points. Hence, as discussed previously, there is a trend to move toward decentralized or distributed control philosophy. The development of smart inverter is one step toward this goal. As defined in California Rule 21, seven autonomous control functions, including dynamic Volt-VAR controls and fixed PF, have been defined for smart inverter operation. In the full revision of IEEE 1547 [103], five different voltage control modes are proposed, including adjustable constant PF mode, voltage-reactive power mode, active power-reactive power mode, adjustable constant reactive power mode, and voltage-real power mode. Some of these control functions/modes are autonomous, meaning that without the need of upper-level supervisory control, some will need commands from an upper-level controller. This has left a gap for control coordination and system global optimization. California Smart Inverter Working Group approached this in Phase 2, requiring communication capability for smart inverters, and in Phase 3, defining advanced functions under communications. However, these existing activities do not address the challenges of highpenetration solar PV and the issues of DERMS/ADMS control scalability. With the aforementioned background and within the common practice of DERMS/ADMS control framework, the team proposes a DVC, which coordinates responsive/adaptive HVAC load control with the local smart inverter connected at the same feeder nodes to achieve an optimal voltage control solution in terms of high consumer energy efficiency, reduced feeder power loss, maximal renewable energy use, and the least cost to achieve the same goal. The proposed DVC can also be integrated into a centralized control framework (e.g., IVVC from ADMS) or a cooperative control framework with additional voltage control requirements (i.e., the control amount to guarantee downstream node voltage stays within the ANSI limits in a radial power system) from the downstream feeder node.

\section{A.2.4 Concept of a New DVC}

As illustrated in Figure A-18, this new DVC can be associated with any node in a distribution feeder equipped with a smart PV inverter and adaptive building HVAC load controller. This feeder node can be connected to an upstream feeder node and/or a downstream feeder node. The node DVC will receive one or more voltage control commands from either a central controller or multiple voltage controllers at the downstream neighboring nodes. This control signal is the delta-V required to guarantee the global optimal voltage quality and can be treated as a tertiary control signal. The DVC serves as a local secondary voltage controller to optimize and coordinate the voltage control efforts of smart PV inverters and load controls. For the smart inverter, the DVC can dispatch either the reactive power reference or an optimal voltage reference, which will initiate a corresponding voltage control mode within the smart inverter. For the load controller, the DVC will dispatch a delta-P that represents the required load change amount. The DVC engine is a local optimization algorithm and solver that is much smaller than the IVVC problem in the centralized control and faster for real-time and online control. The primary objective is to find the optimal voltage reference setting for the current node without considering the down-streaming nodes' voltage requirements. This small voltage optimization and control problem will be solved at timescales of 1-5 min and will provide the voltage or reactive power reference to the smart inverter primary controller. 


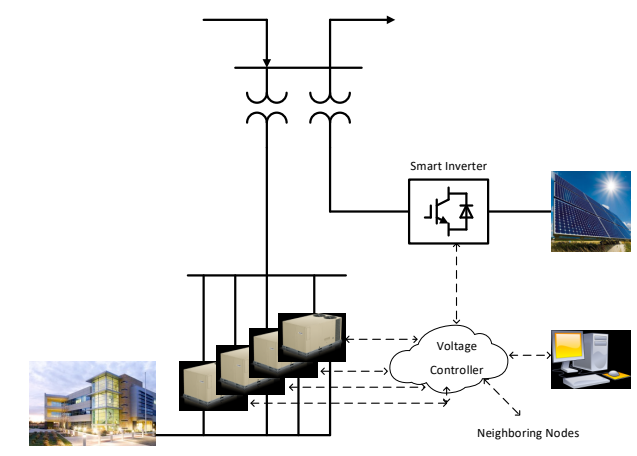

Figure A-18. System hardware architecture of the DVCs.

\section{A.3 IEEE 13-NODE TEST FEEDER DISTRIBUTION-LEVEL PV PENETRATION ANALYSIS}

As the solar PV system price continues to drop, the number and size of PV installations continue to increase, leading to a new era of high-penetration solar in the distribution system. Despite the economic and environmental benefits, high-penetration solar PV scenarios have been observed [29, 111] to impact the grid in various aspects and impose challenges for distribution network operators. Particularly, feeder voltage impact is attracting much of the attention. For example, because of inherent variability, PV generation can be greater than the local demand, and the excess power could produce reverse power flows in a distribution feeder. This would not only lead to voltage rise but also potentially violate voltage limits along the feeder. The impacts can further reduce the lifetime of the transformer due to rapid tap changing adjustments.

Depending on the solar PV penetration level, the following potential voltage impacts might occur in the distribution feeder buses, which are crucial for distribution system planning and design:

- steady-state over-voltage exceeding admissible operational conditions (ANSI voltage limit, 5\% above nominal),

- transient voltage spikes, and

- $\quad$ significant voltage variability resulting in poor voltage regulation and increased cycling and stress on voltage control equipment, such as LTCs, line regulators, and switched capacitor banks.

The impact of solar power on a distribution feeder voltage profile can be analyzed by performing steadystate power flow analyses, which assume constant load and solar power generation. However, to quantify the operational impact on distribution substation transformer LTCs or feeder voltage regulators, the analysis must account for the time variability of load and solar power at the seconds or minutes timescale. This requires high-resolution load and solar power generation data and dynamic or QSTS simulations [112].

Other works [29-30,111] have reported the steady-state voltage impacts, but little work has been done on the lifetime impact on feeder voltage regulation devices and mitigation options. Recently, adaptive building HVAC controls were proposed to mitigate the time-dependent voltage impacts of solar PV power. However, to quantify the control performance, the baseline analysis of nodal voltages and feeder LTC operation behaviors under different solar penetration levels must be understood. This section presents the analysis performed to understand the impact of PV penetration on LTCs. For clarification, in this report, the capacity penetration level is adopted and defined as the nameplate capacity of the combined solar PV inverters on a circuit divided by the peak annual load on that circuit. 
In the following analyses, the solar PV inverters are assumed to be complaint with IEEE 1547-2003 and not equipped with advanced functions, such as dynamic Volt/VAR control or solar power curtailment functions. The Electric Power Research Institute's (EPRI's) OpenDSS ${ }^{3}$ and Sandia National Laboratories' $\mathrm{GridPV}^{4}$ tools were used for the QSTS simulations. The IEEE 13-node distribution test system was chosen for the analyses due to its highly unbalanced nature and the simplicity of the system.

Regarding to the modeling tools, the team chose EPRI's OpenDSS because it is open source, is widely recognized within the power industry, and can perform different power system analyses. The following paragraph is excerpted from the OpenDSS website:

The OpenDSS is a comprehensive electrical power system simulation tool primarily for electric utility power distribution systems. It supports nearly all frequency domain (sinusoidal steady state) analyses commonly performed on electric utility power distribution systems. In addition, it supports many new types of analyses that are designed to meet future needs related to smart grid, grid modernization, and renewable energy research. The OpenDSS tool has been used since 1997 in support of various research and consulting projects requiring distribution system analysis. Many of the features found in the program were originally intended to support the analysis of distributed generation interconnected to utility distribution systems and that continues to be a common use. Other features support analysis of such things as energy efficiency in power delivery and harmonic current flow. The OpenDSS is designed to be indefinitely expandable so that it can be easily modified to meet future needs.

Electricité de France (EDF) conducted a study ${ }^{5}$ to compare OpenDSS with another software FACE in 2010. The conclusion was that "OpenDSS is able to achieve good accuracy within a rather short time. OpenDSS appeared to have better results than FACE, which had been studied by EDF R\&D." In this work, the team also compared its steady-state power flow analysis results with the ones for the IEEE 13Node Test Feeder [113] and confirmed very good matching when using the OpenDSS solver.

GridPV is a MATLAB toolbox developed by Sandia National Laboratories that provides MATLAB-style visualization abilities of the power flow analysis results from OpenDSS through a COM interface. The IEEE 13-Node Test Feeder, ${ }^{6}$ shown in Figure A-19, was used in this work to examine the voltage impact of different solar PV penetration levels. This is a relatively small feeder, and it exhibits many interesting characteristics [113], such as short and highly loaded $4.16 \mathrm{kV}$ feeder, substation voltage regulator consisting of three single-phase LTCs at Bus 650, one three-phase fixed capacitor at Bus 675 and one single-phase fixed capacitor at Bus 611, and nine load buses connected into different phases. The crossbars in the figure depict the number of phases and demonstrate that the feeder is unbalanced.

\footnotetext{
${ }^{3} \mathrm{http}: / /$ smartgrid.epri.com/SimulationTool.aspx

${ }^{4}$ https://pvpmc.sandia.gov/applications/gridpv-toolbox/

5 http://smartgrid.epri.com/doc/EPRI\%20Smart\%20Grid\%20Advisory\%20Update\%202010_11_16.pdf

${ }^{6}$ https://ewh.ieee.org/soc/pes/dsacom/testfeeders/
} 


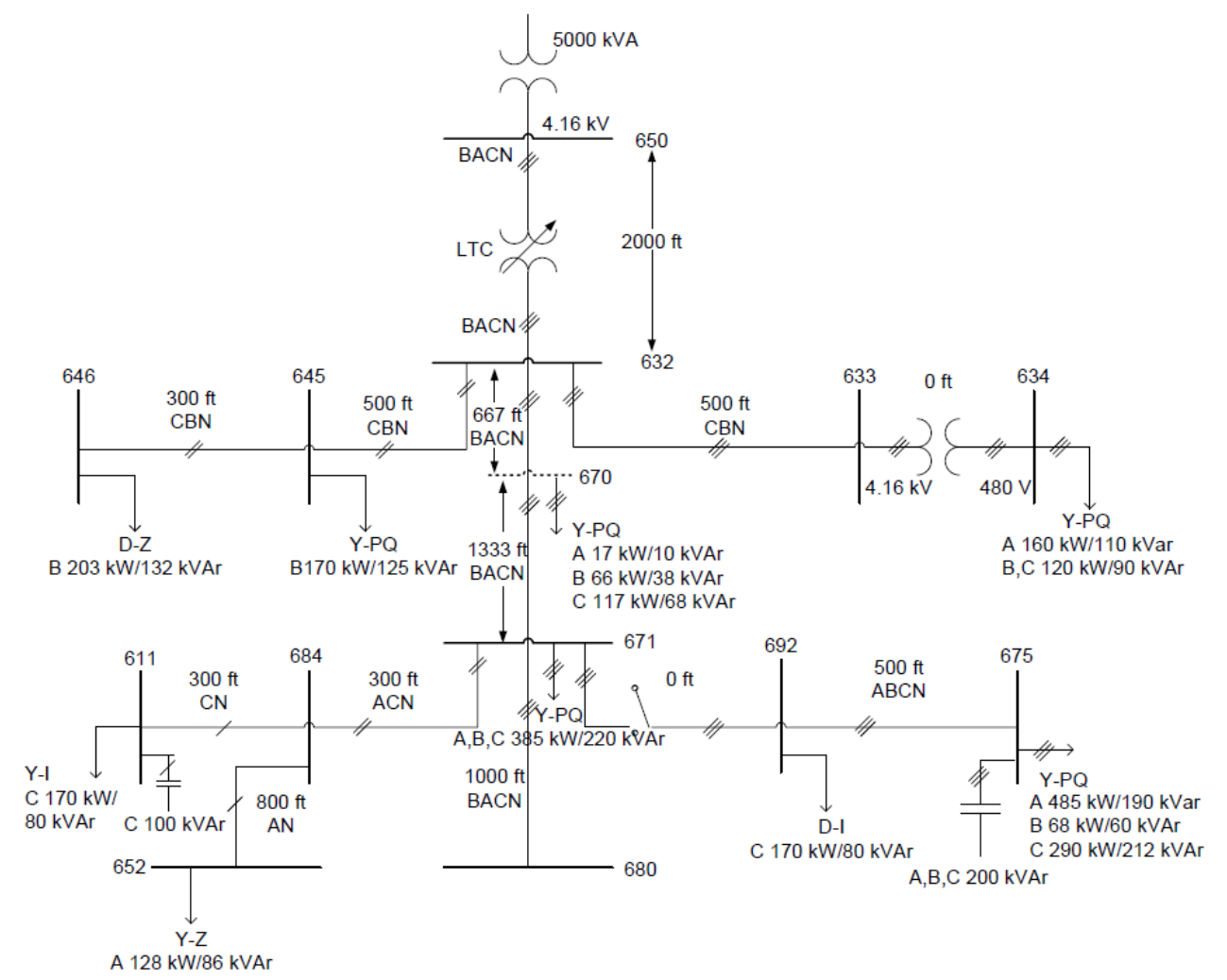

Figure A-19. One-line circuit of IEEE 13-Node Test Feeder.

The total peak load demand in this feeder is $4,053.6 \mathrm{kVA}$ with $3,466 \mathrm{~kW}$ of real power and 2,102 $\mathrm{kVar}$ of reactive power. The peak load PF is 0.85 . This section presents the results of a centralized three-phase PV system with inverters interconnected into Bus 680 . This represents the worst-case scenario for voltage impact analyses.

\section{A.3.1 Annual Load and Solar Power Profiles}

For time series simulation, hourly load data for a 1 year period were collected with a linear interpolation to increase the data resolution to $1 \mathrm{~min}$. All the single-phase and three-phase loads use individual load profiles, although they show similar patterns. In Figure A-20, the normalized hourly load profile for single-phase and three-phase loads is plotted for 8,760 h. 

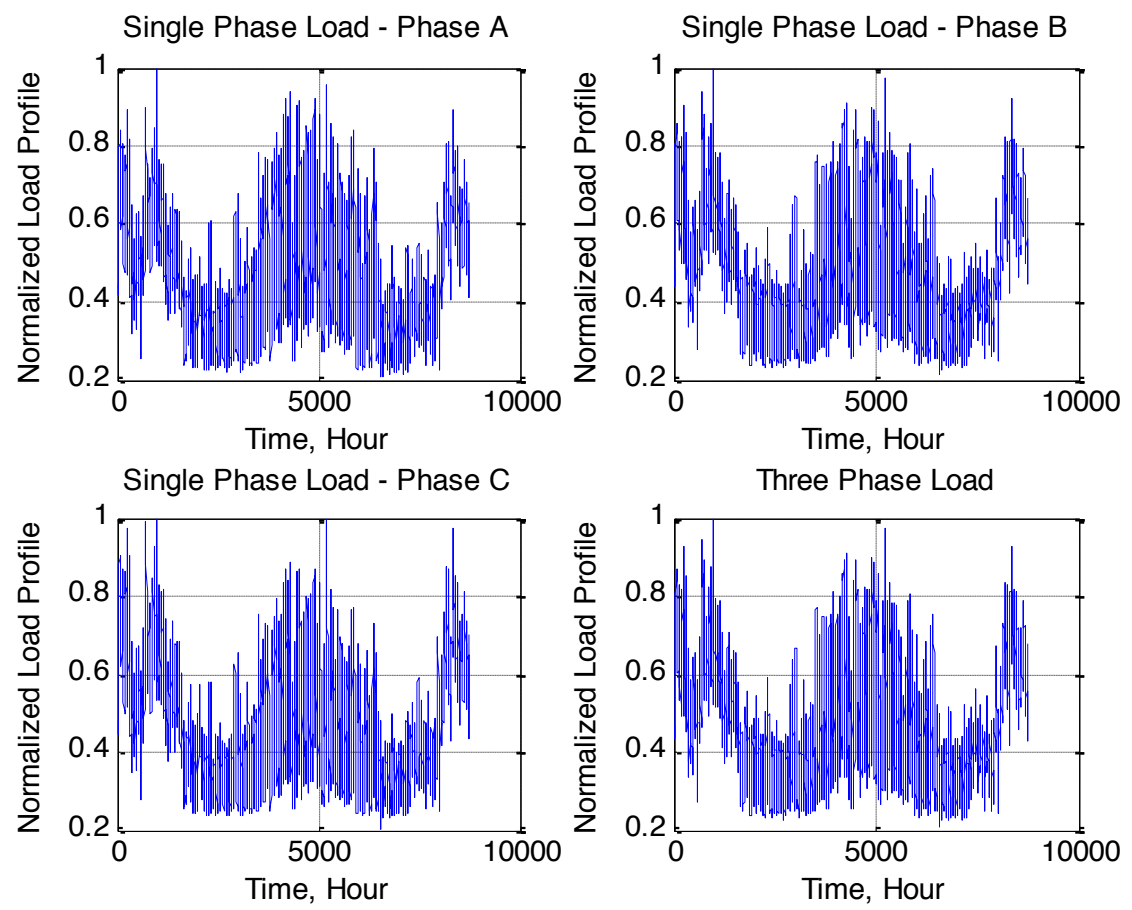

Figure A-20. Annual load profile for single-phase and three-phase loads.

Similarly, high-resolution solar power data are required to demonstrate the voltage impact at the seconds or minute timescale. For this study, the 5 min Tennessee's solar generation data from National Renewable Energy Laboratory's (NREL's) solar power data for integration studies ${ }^{7}$ were used. Normalized 5 min solar power data are plotted in Figure A-21. Again, linear interpolation was used to obtain $1 \mathrm{~s}$ resolution data for the simulation.

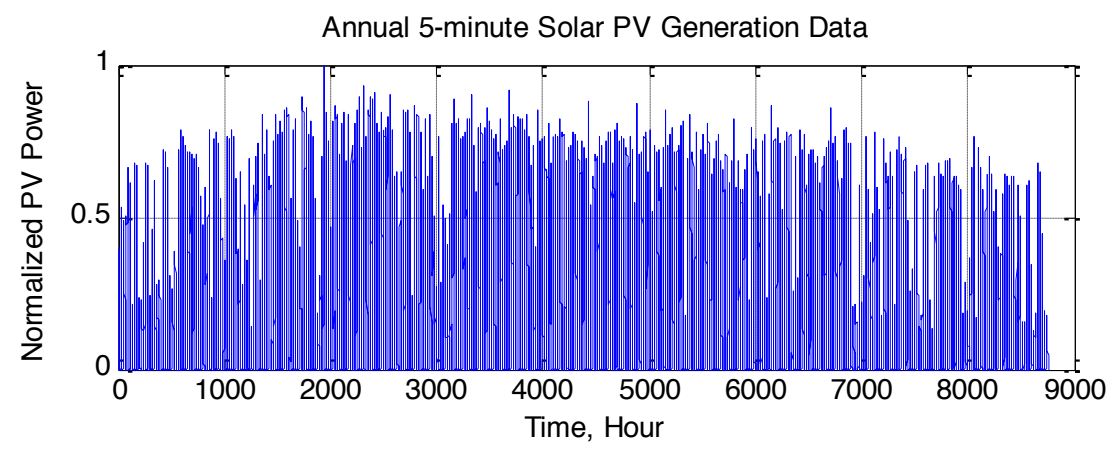

Figure A-21. Annual solar PV generation profile.

\section{A.3.2 Solar Power Production at Different Penetration Levels}

As mentioned previously, the solar PV penetration level is defined by the maximum or rated PV inverter power capacity divided by the feeder peak load. For the IEEE 13-Node Test System, these data are given in Table A-1 for different penetration levels.

\footnotetext{
${ }^{7}$ http://www.nrel.gov/grid/solar-power-data.html
} 
However, the real-time solar power generation depends on the solar irradiation and ambient temperature, which will be much less than the rated nameplate power. In our study, we used real solar power measurement data collected from NREL's previous integration studies and normalized as the solar power generation profile. For a typical week in August, the solar PV power generations for different defined penetration levels are shown in Figure A-22, and the approximate peak PV power is also listed in Table A-2 with equivalent penetration levels.

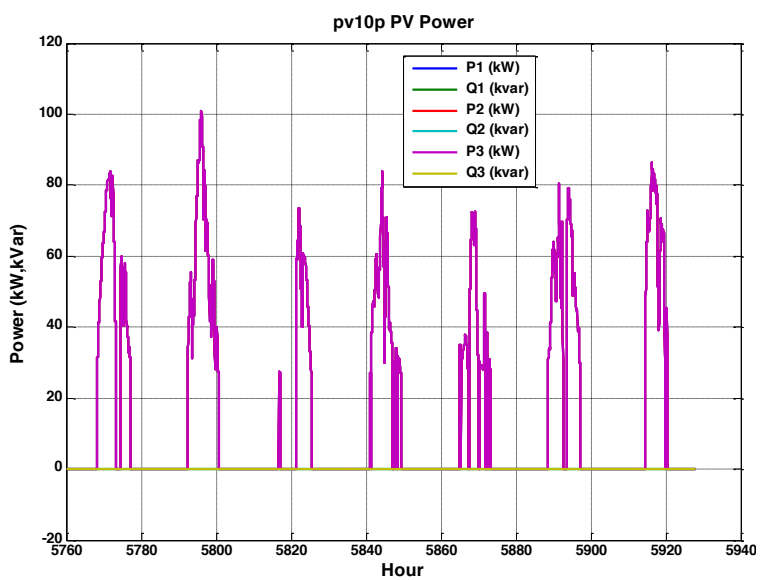

(a) $10 \%$ PV penetration.

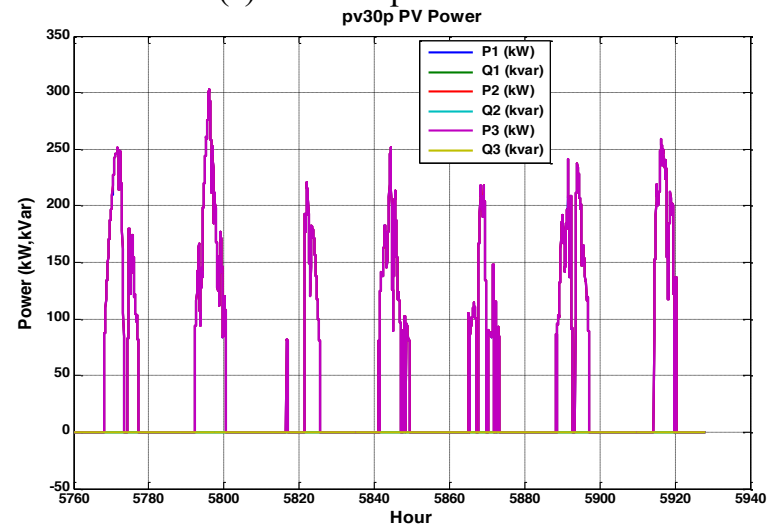

(c) $30 \%$ PV penetration.

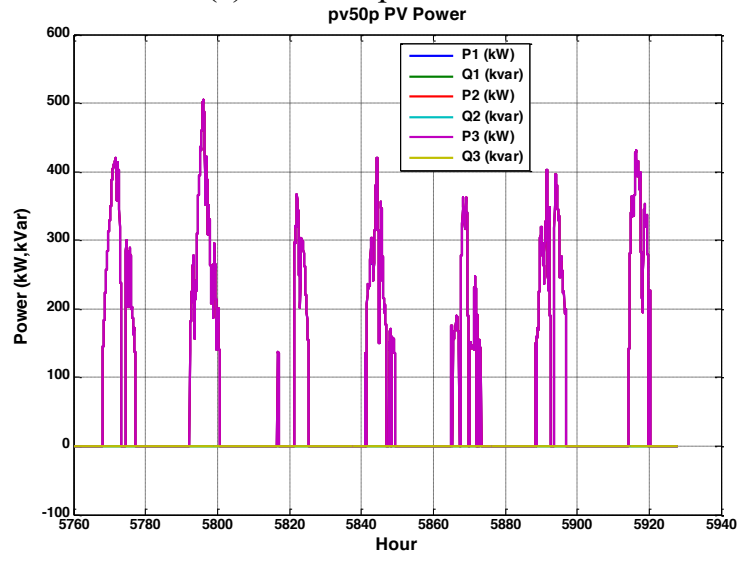

(e) $50 \%$ PV penetration.

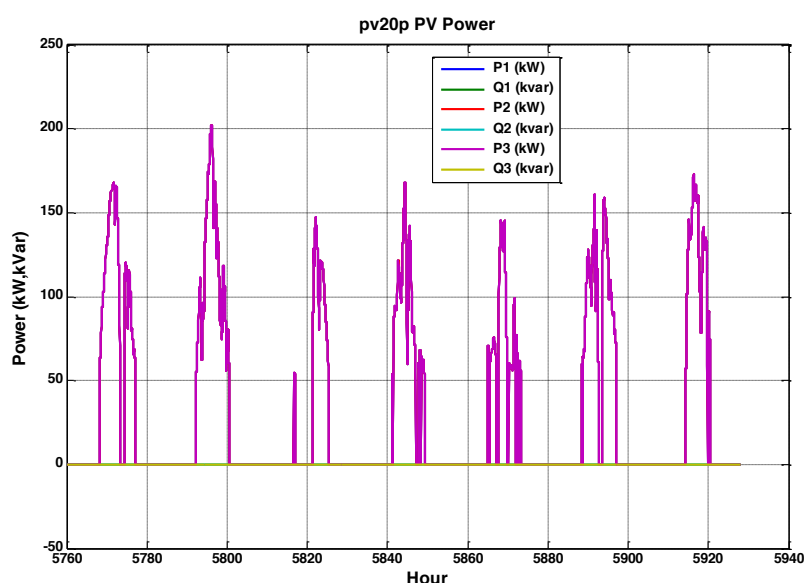

(b) $20 \%$ PV penetration.

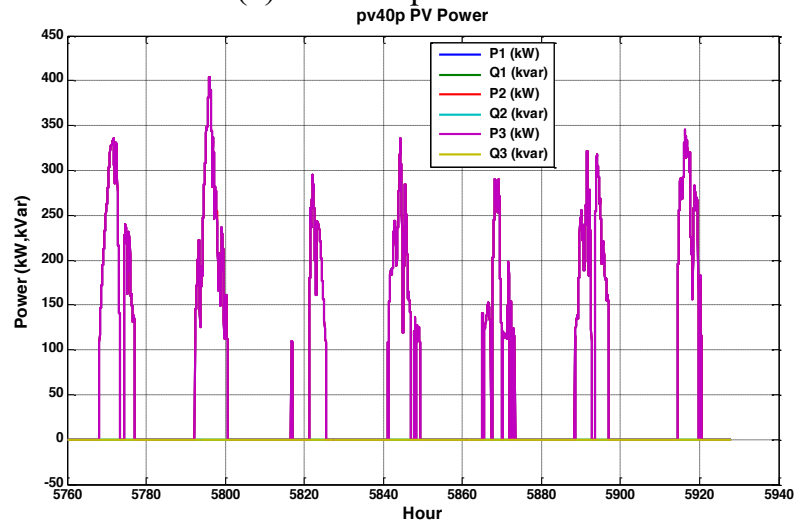

(d) $40 \%$ PV penetration.

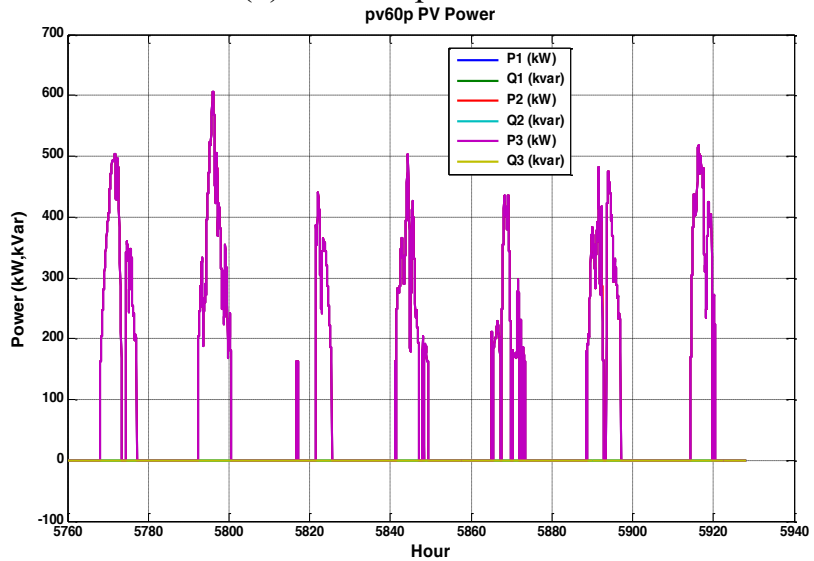

(f) $60 \% \mathrm{PV}$ penetration. 


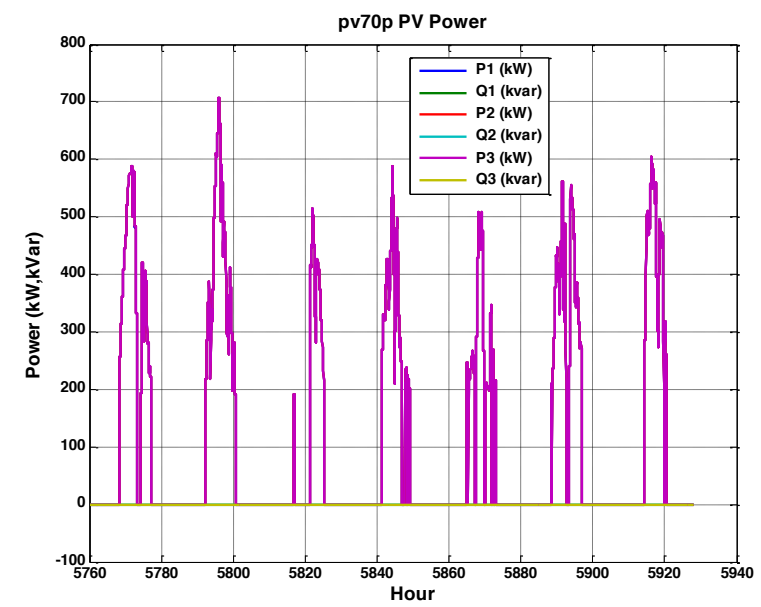

(g) $70 \%$ PV penetration.

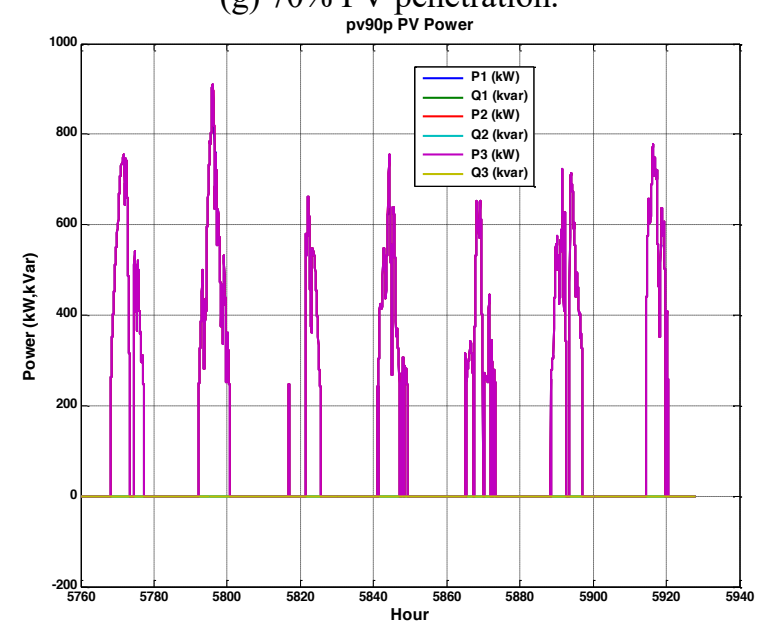

(i) $90 \% \mathrm{PV}$ penetration.

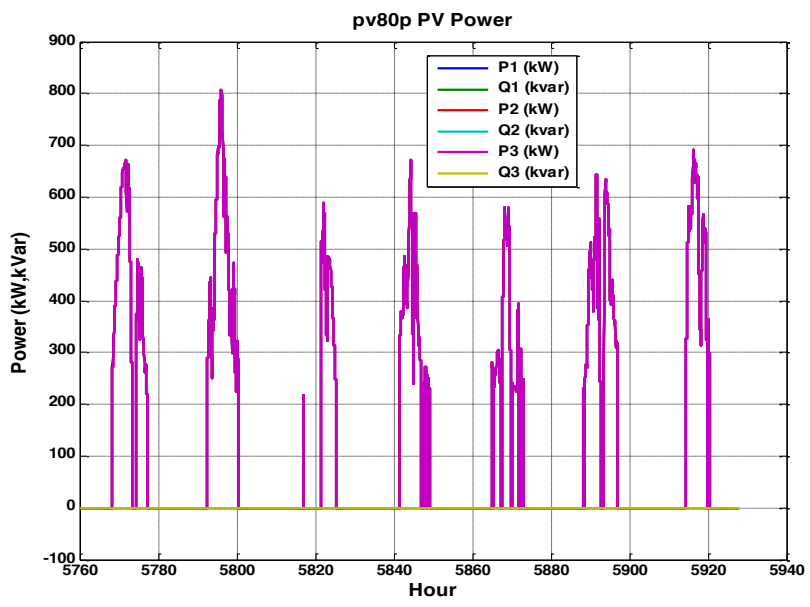

(h) $80 \%$ PV penetration.

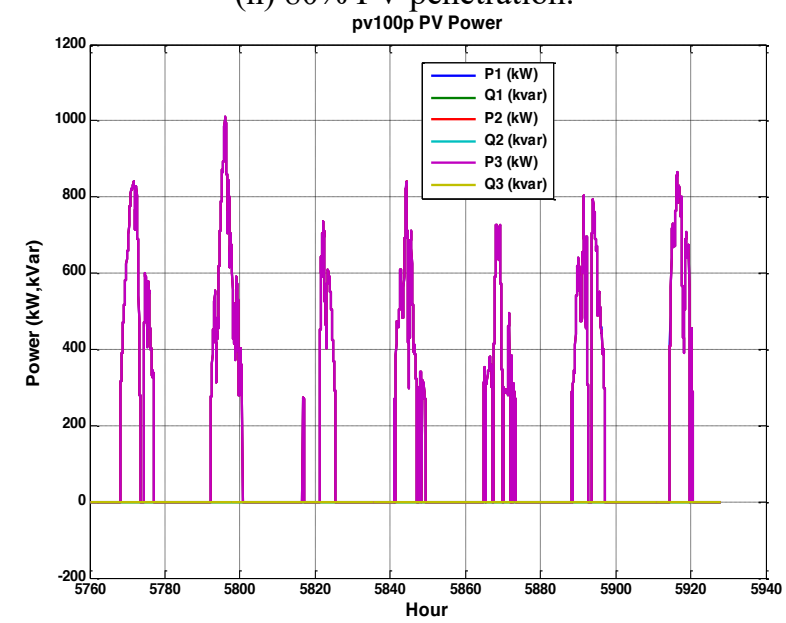

(j) $100 \%$ PV penetration.

Figure A-22. Solar PV generation in 1 week in August at different penetration levels.

Table A-2. Solar power generation at different penetration levels.

\begin{tabular}{cccc}
\hline $\begin{array}{c}\text { Defined } \\
\text { penetration } \\
\text { level (\%) }\end{array}$ & $\begin{array}{c}\text { Maximum PV } \\
\text { inverter power } \\
\text { capacity (kVA) }\end{array}$ & $\begin{array}{c}\text { Approximate peak } \\
\text { PV power generation } \\
\text { (kVA) }\end{array}$ & $\begin{array}{c}\text { Equivalent penetration } \\
\text { level (ratio to peak } \\
\text { load) (\%) }\end{array}$ \\
\hline 10 & 405 & 240 & 6 \\
20 & 811 & 450 & 11 \\
30 & 1,216 & 750 & 18 \\
40 & 1,622 & 900 & 22 \\
50 & 2,027 & 1,200 & 29 \\
60 & 2,432 & 1,500 & 37 \\
70 & 2,838 & 1,800 & 44 \\
80 & 3,243 & 2,000 & 49 \\
90 & 3,649 & 2,300 & 56 \\
100 & 4,054 & 2,400 & 59 \\
\hline
\end{tabular}




\section{A.3.3. Impact on Steady-State Voltage Profile}

In this study, the test feeder circuit was modeled by using EPRI's distribution analysis tool, OpenDSS. A steady-state power flow analysis was performed for the cases of no solar PV and 50\% solar PV penetration. Figures A-23 and A-24 show the steady-state voltage profile for no PV and 50\% PV, respectively. From the results, the voltage at all the phase feeder ends is elevated, particularly for Phase B (red) because of the fixed capacitor bank at Bus 675. The voltage in Phases A and C also increased because of the reverse power flow from solar PV, which increased the voltage of Bus 671.

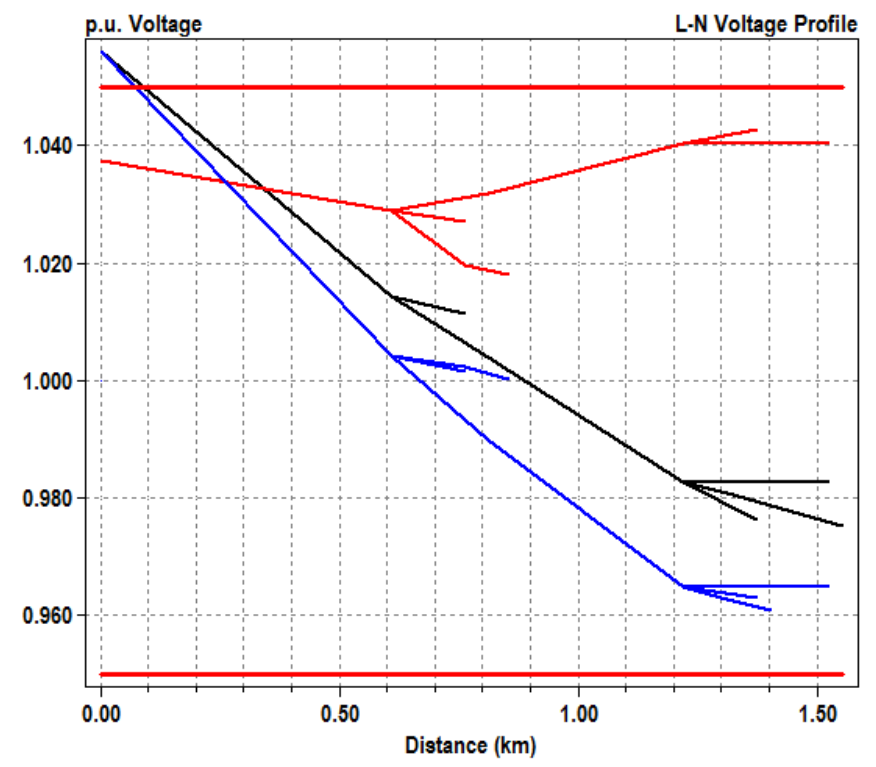

Figure A-23. Steady-state voltage profile with no solar PV.

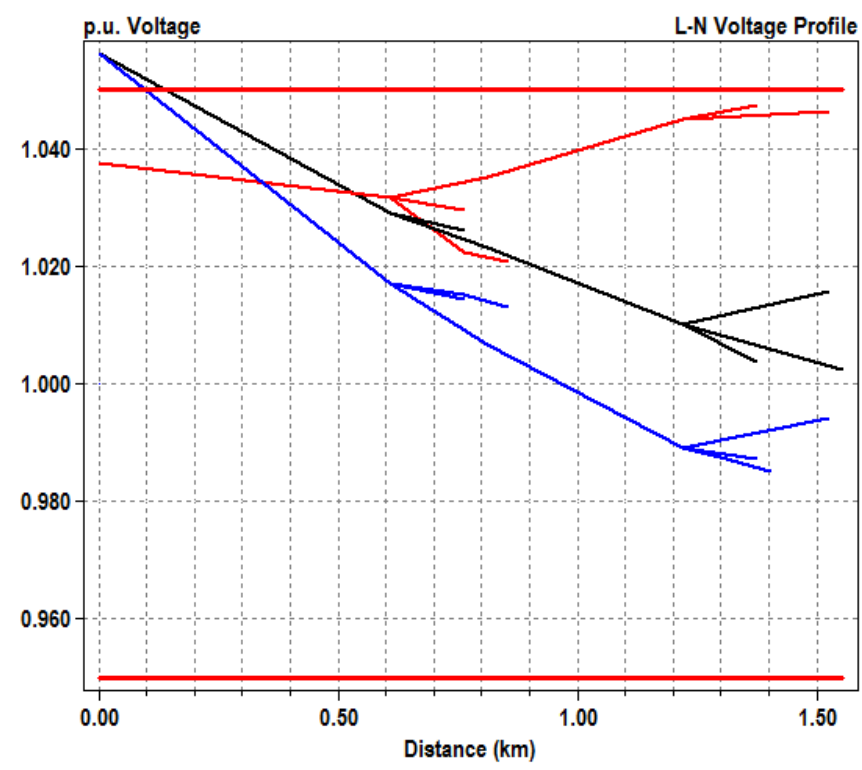

Figure A-24. Steady-state voltage profile with $50 \%$ solar PV penetration. 


\section{A.3.4 Impact on Substation Voltage, PF, and Capacitor Voltage}

To analyze the impact of solar power minute-to-minute fluctuations on the feeder and bus voltages, QSTS simulations are needed. In this work, Sandia National Laboratories' GridPV toolbox for MATLAB was used, which calls for OpenDSS for quasi-steady-state power flow analysis (i.e., "multiple snapshots") through OpenDSS's COM object interface.

The QSTS simulation was performed for a typical day in August with a $1 \mathrm{~s}$ time step. The load and solar power data were interpolated within the OpenDSS software to obtain the $1 \mathrm{~s}$ resolution data. A threephase PV inverter was interconnected to Bus 680 with a rated capacity of 4,054 kVA, which namely represents $100 \%$ penetration based on the feeder's annual peak load. In real-world scenarios, depending on the solar irradiation and temperature, the solar power generation can be much lower, as shown in Figure A-25 for an arbitrarily selected day, which has a peak power of roughly about 3,000 kW for three phases. Based on the earlier assumption of no Volt/Var control, the PV inverter only generates real power and does not provide reactive power.

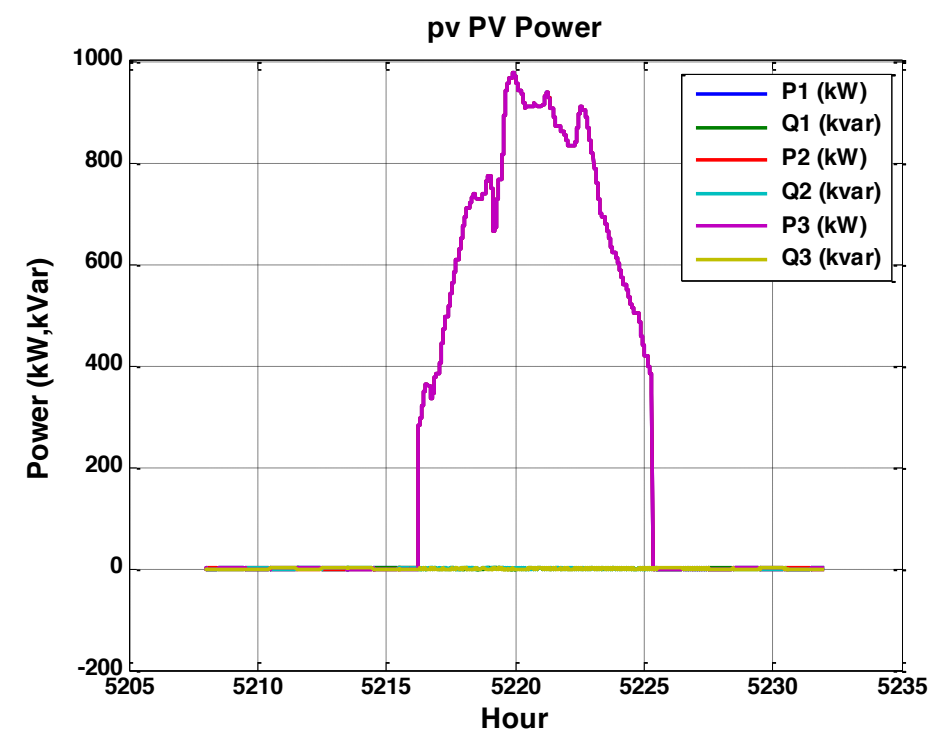

Figure A-25. Solar PV generation for a typical day in August.

For the same day, the substation voltage, net load, PF, and the voltages of two capacitors are plotted in Figures A-26 through A-30. As shown in the figures, during the middle of the day when solar PV is generating real power, the substation net real power load is reduced. However, for the same time period, the reactive power load increasingly results in a dip in substation PF to approximately 0.1 and a slightly lower substation voltage due to the operation of LTCs. The voltage effects on two fixed capacitors are not dramatic because the PV inverter does not generate reactive power. 


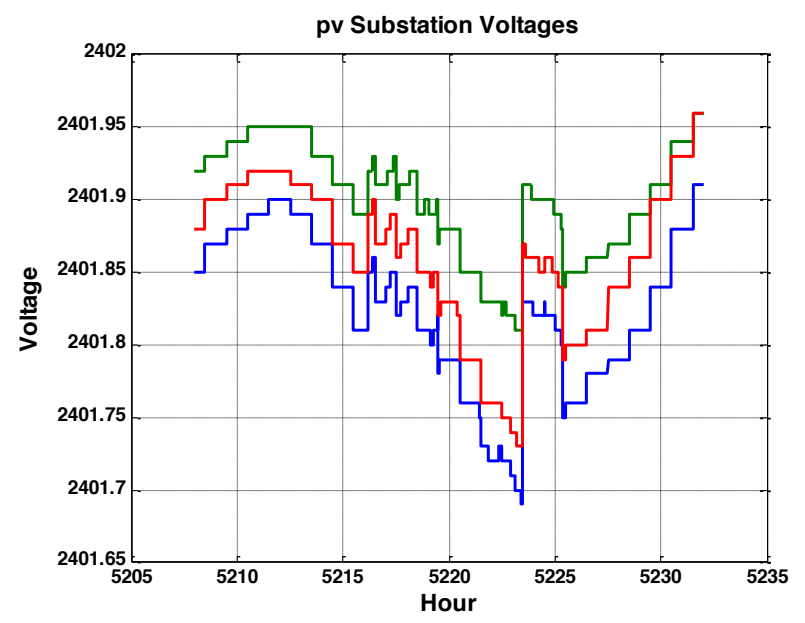

Figure A-26. Transformer secondary voltage.

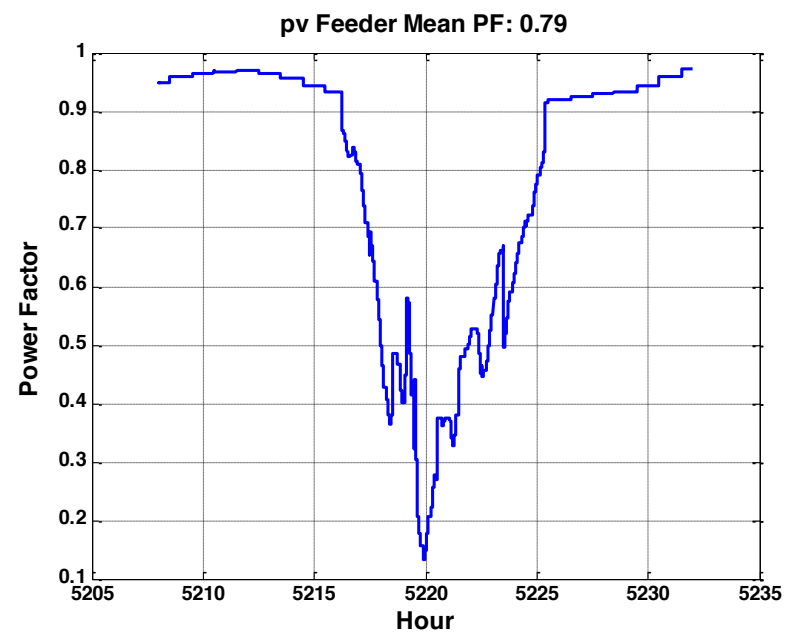

Figure A-28. Substation feeder PF.

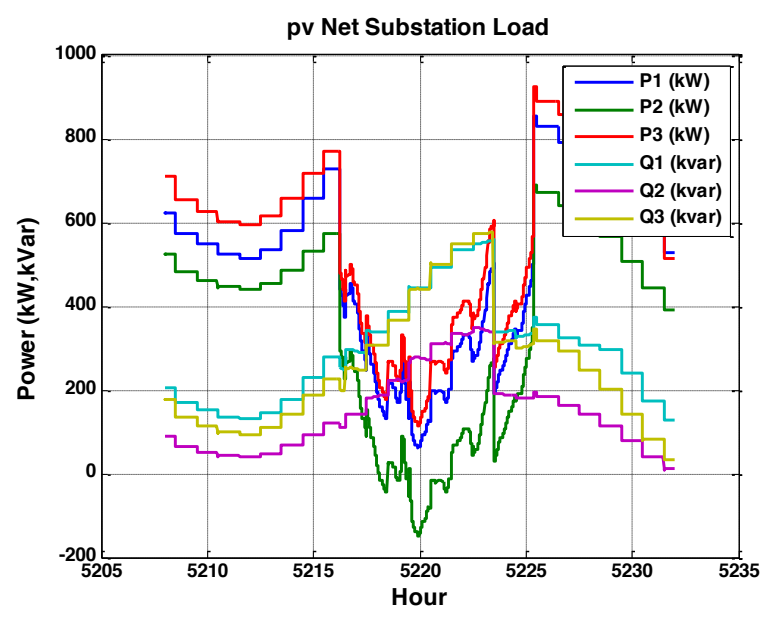

Figure A-27. Substation net load.

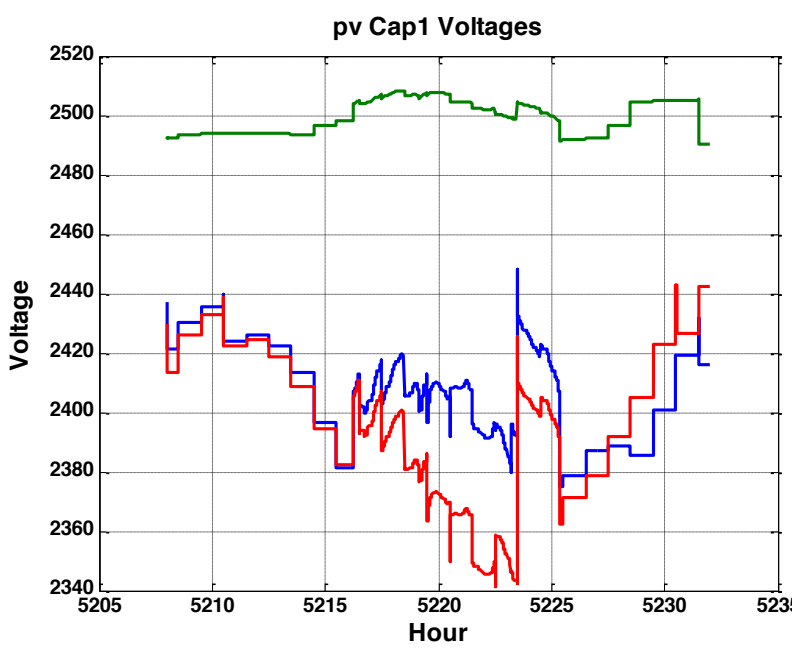

Figure A-29. 3申 cap. voltage at Bus 675. 


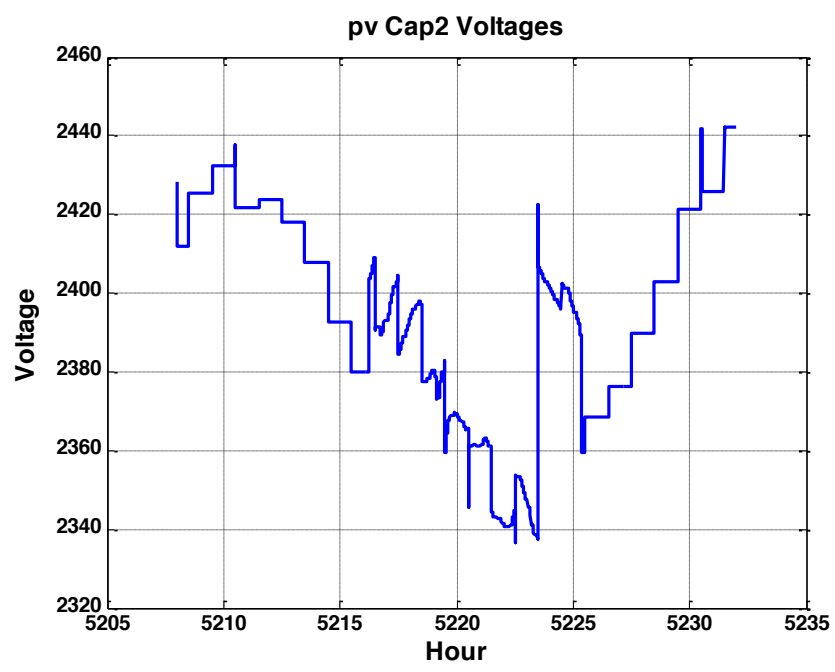

Figure A-30. Single-phase capacitor voltage at Bus 611 (Phase C).

\section{A.3.5 Impact on Voltage Regulator LTC Operation}

The impact on voltage due to PV variations could increase the tap change operation counts of the feeder voltage regulator or substation transformer LTCs. This was verified by the QSTS simulations for 1 week with a 1 min time step at 50\% solar PV penetration, as shown in Figure A-31. Solar PV power can change dramatically within a very short time due to passing clouds. EPRI has measured up to $1 \mathrm{MW}$ per $20 \mathrm{~s}$ in the field. A worst-case scenario would be the ramping of solar PV output from zero to the maximum output power in tens of seconds, which is faster than the feeder regulation equipment can respond because voltage regulation equipment typically has a time delay of $45-90 \mathrm{~s}$, so this will result in potential overvoltage exceeding the regulation bandwidth.
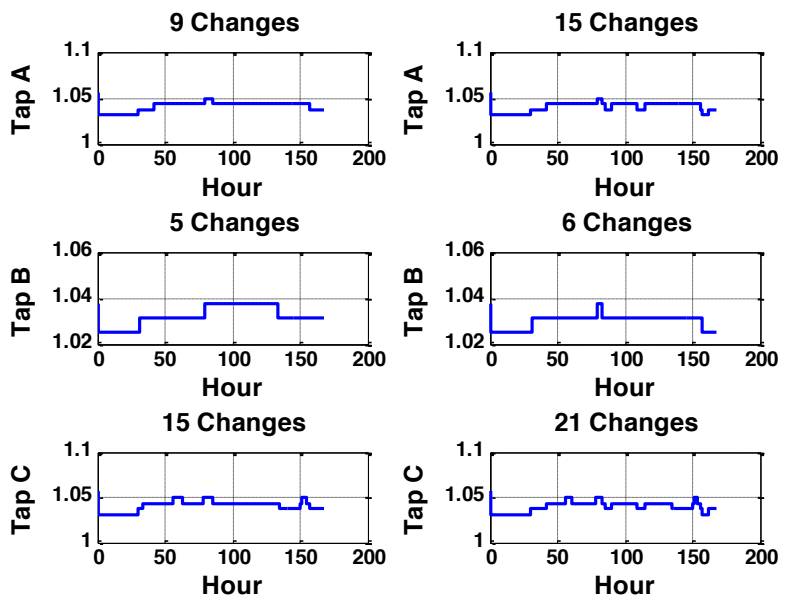

Figure A-31. Tap changes (left: no PV, right: with 50\% PV).

To investigate the effects of different solar PV penetration levels, multiple simulations are performed for a 1 year period at a 1 min time resolution. The accumulated tap changes over a 1 year period are shown in Figure A-32 for no PV and with PV penetration from 50 to $100 \%$ at $10 \%$ increases. In this figure, the total tap changes significantly increase from 600 times to 1,000 times for Phase A, 240 times to 580 times for Phase B, and 750 times to 1,100 times for Phase C with an increase in PV penetration from 0 to $100 \%$ 


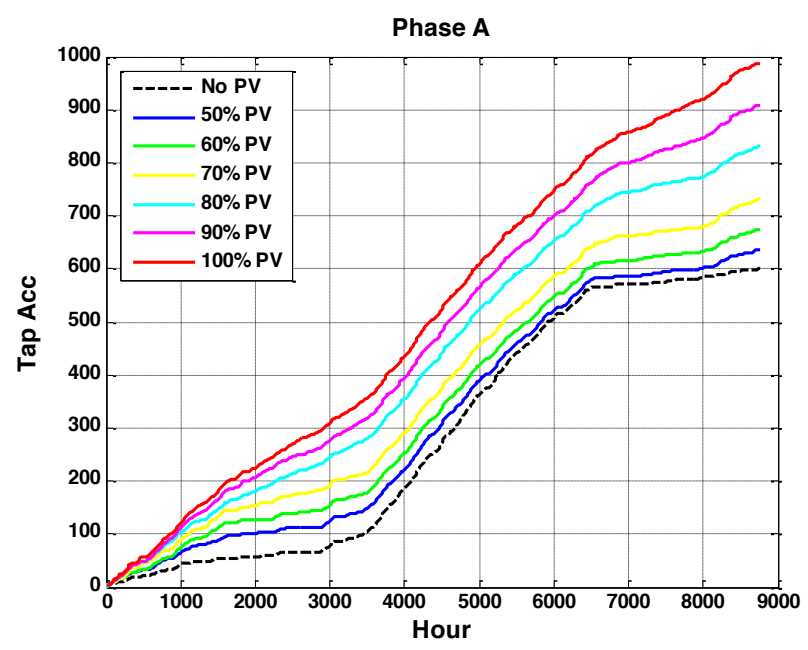

(a) Cumulative tap changes for Phase A.

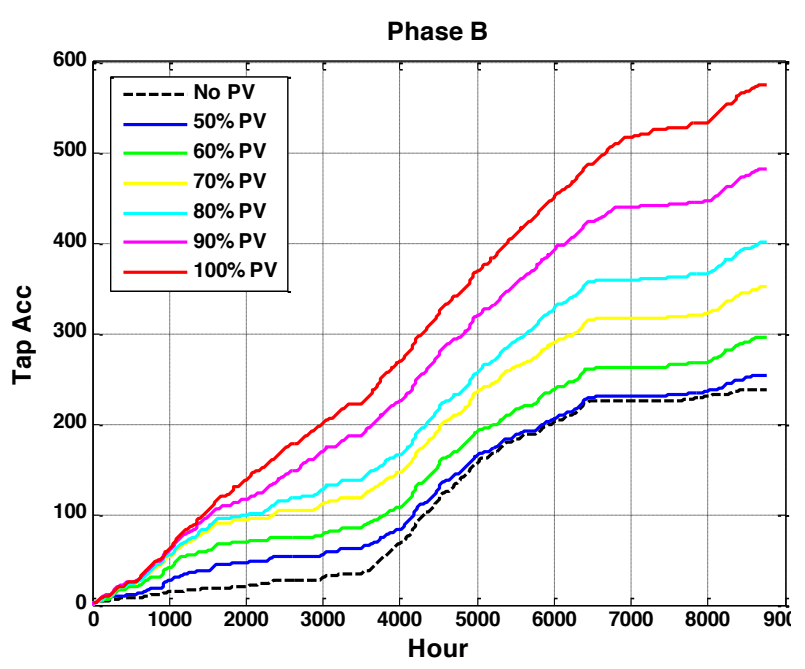

(b) Cumulative tap changes for Phase B.

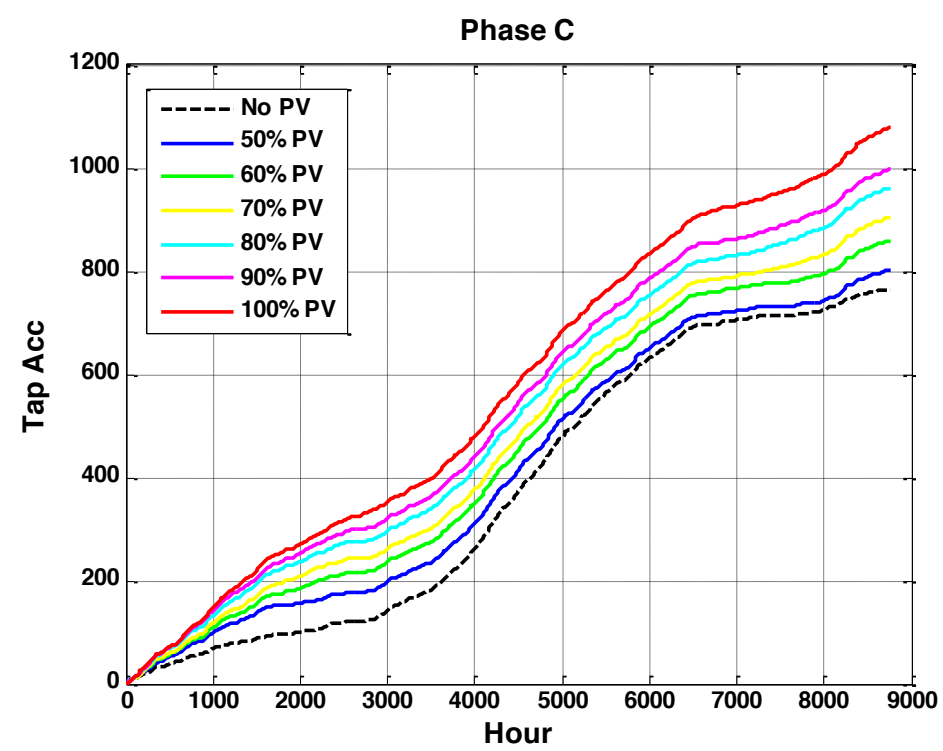

(c) Cumulative tap changes for Phase C.

Figure A-32. Cumulative regulator tap changes over a 1 year period for different solar PV penetration levels.

\section{A.3.6 Smart PV Inverter with Voltage Regulation Function}

Traditionally, solar PV inverters only provide basic energy feeding functions (i.e., inject real power only to the power grid based on the MPPT at different solar irradiances and ambient temperatures). IEEE Standard 1547-2003 and UL 1741 require a solar PV inverter to trip off in case of abnormal grid voltage and frequency. 
With increased solar installation penetration in distribution grids, the operation reliability and stability, as well as power quality issues, are becoming eminent. A smart inverter concept $[70,102]$ was proposed that calls for advanced control functions to provide ancillary grid services, including dynamic reactive power control and voltage regulation [105], for solar PV inverters. Currently, IEEE standard 1547 is under full revision to allow or mandate solar PV inverters for these advanced grid voltage regulation functions.

\section{A.3.7 Dedicated Volt/Var Compensation Devices}

Conventional distribution system operation has been treated as a Volt/Var control problem over the years, using the distribution transformers equipped with LTCs, feeder voltage regulators, and fixed or switched capacitor banks. These mechanical devices are slow reacting and can be controlled only by certain voltage steps or ratios. Thus, they are handicapped to respond to the fast dynamics introduced by increasing renewable energy systems. SVCs or other flexible AC transmission system-type devices (e.g., FACTS) have been developed to cope with this issue by using the solid-state switches to provide continuous reactive power compensation. These systems are typically costly and are under replacement due to using the aged and old-fashioned semiconductor devices (e.g., gate turn-off thyristors).

With the development of multilevel power electronics converters and high-voltage, high-efficiency wide bandgap semiconductor devices, solid-state transformers [114] or partial solid-state transformers (also called controllable transformers [115]) are developed to offer combined power flow and voltage controls. Small-scale distributed Volt/Var compensation devices that use power electronics, called grid edge technologies [116], are also emerging to provide distributed voltage management.

\section{A.4 DISTRIBUTED VOLTAGE CONTROL}

\section{A.4.1 Background on DVC}

Power or load flow computation has been a traditional problem for the transmission system operator. This problem computes voltage magnitude and angle at each bus, real and reactive power flows for all equipment interconnecting the buses, subjecting to the constraints of power flow balance, bus voltage magnitude close to rated value or within limits, generator P and Q within specified limits, line and transformers not overloaded. The power flow analysis assumes that the power system is operated normally and is steady-state with balanced three phases by using a single-line diagram. The classical algorithms or solvers include Gauss-Seidel method, Newton-Raphson method, and Fast Decoupled method.

More commonly, the power flow will be an OPF problem - the first of which was formulated in 1962 by Carpentier [117-118] — which is the heart of independent system operator's power market and is solved every year for system planning, every day for day-ahead markets, and every hour and even every 5 min for spot markets. The typical objective functions for the OPF optimization problem are minimizing fuel costs; minimizing system losses or minimizing generation or maximizing transfer; minimizing load shedding, thereby improving reliability; minimizing control actions, hence extending device lifetime; and so on. Besides the aforementioned constraints, contingency constraints might be also considered to form a security constrained OPF problem.

This problem is difficult to solve due to its nonconvex nature and its challenges on computation power, speed, and solution accuracy (i.e., the optimality). Distribution system or the new DSO model typically does not perform a complete OPF analysis because of the nature of distribution system in North America (i.e., unbalanced three phases and high $\mathrm{R} / \mathrm{X}$ ratio making $\mathrm{P}$ and $\mathrm{Q}$ hard to decouple). However, a special case in distribution systems is the VVO, which is the distribution voltage control problem. 
The primary goal for distribution voltage control is to maintain the nodal voltages within the ANSI limits. Other objectives or criteria are to minimize power losses on the feeders, reactive power adjustments on nodes or total reactive power exchange, the number of switching operations, voltage deviations from a voltage reference on nodes or voltage differences among nodes, and so on. With the introduction of DGs and penetration of solar PV inverters, minimizing renewable power generation curtailment and maximizing DG active power output are also goals.

\section{A.4.2 DVC Methods}

Previously, the team systematically surveyed the topic of distribution VVO from power quality and energy efficiency requirements, existing utility practices (SCADA-based and DMS-based solutions), and various control devices, including the utility-owned distribution substation transformer equipped with LTCs, feeder SVRS, fixed or adjustable capacitor banks, power electronic-based D-STATCOM or UPQC devices, and grid-edge technologies (e.g., solar PV inverters). Then, the team summarized different distribution voltage control and communication architecture and briefly evaluated the advantages and disadvantages of various decentralized methods. After observing and identifying the emerging trends of distribution voltage control, the team proposed a new DVC structure that comprises a two-level control structure (Figure A-33) (i.e., the local optimization and primary control level and distributed global optimization and secondary control level). At the primary control level, the HVAC load is tracking solar PV active power, and the PV inverter tracks the load reactive power; at the secondary control level, a DVC determines additional P and Q reference for local controllers, and the DVC communicates with neighboring upstream and downstream nodes. If these nodes do not have communication and/or control capabilities, the DVC will communicate with a central VVO engine (e.g., the IVVC in the ADMS, the distribution substation automation system).

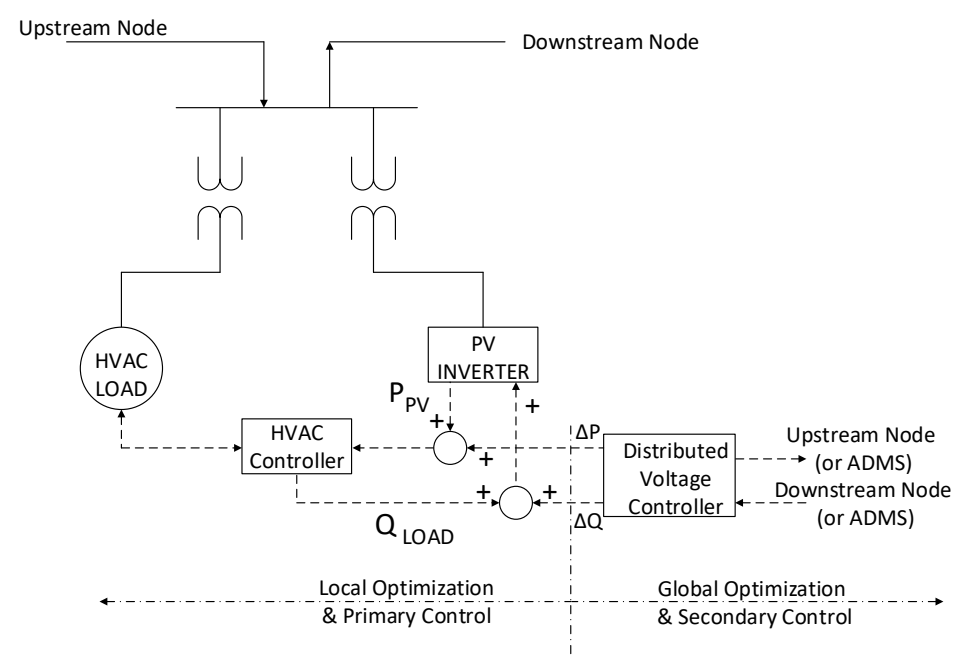

Figure A-33. Proposed DVC control structure.

For a distribution circuit, the team's goals were to (1) minimize the P and Q power flow from the transmission system to alleviate the transmission congestion issue, (2) minimize the total power loss of the distribution circuit for maximized energy efficiency, (3) maximize solar power production to increase renewable power penetration, and (4) minimize the switching actions of LTCs and capacitor banks due to voltage fluctuations for extended equipment lifetime. Scalability, computation, and communication requirements are main factors for choosing proper optimization and control approaches.

First, the team formulated a centralized optimization problem as the baseline approach. The team considered a simple four-node radial distribution system, as shown in Figure A-34, including a 
distribution transformer with LTC at the head bus and load and a PV inverter at each node. The load can be controllable or noncontrollable. For simplification, the team initially omitted any capacitor banks, SVRs, and STATCOM-type voltage control devices. So, the control time hierarchy (Figure A-35) will be as follows:

- Distribution transformer with LTC: Hourly control.

- Adaptive load control: 15 min control.

- Solar PV inverter: 1 min control.

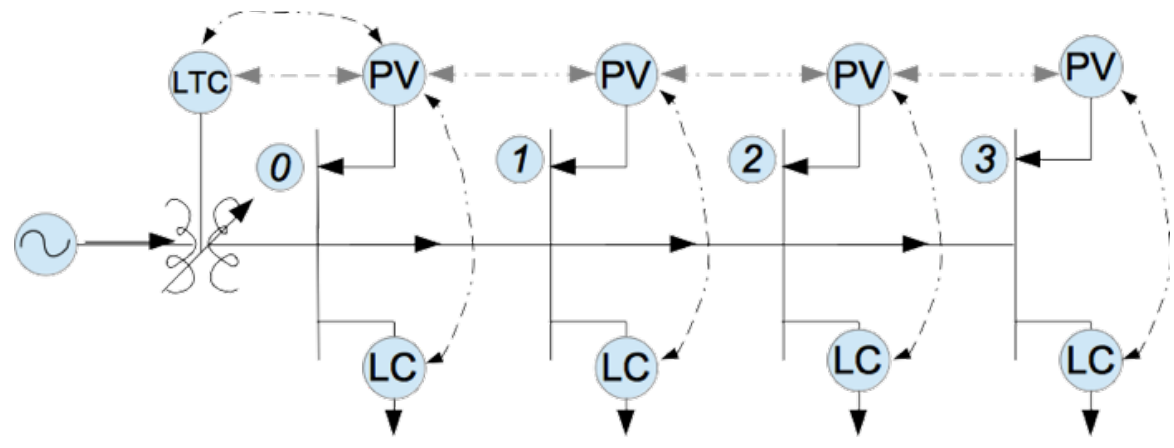

Figure A-34. A simple radial distribution feeder circuit with DVC communication scheme.

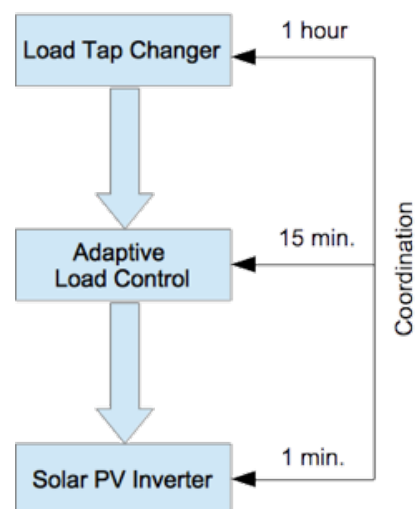

Figure A-35. Control time hierarchy.

The team will minimize an objective function with total system (i.e., real power) loss that is subject to node voltage constraints, PV inverter reactive power constraints, and approximated power flow equations (the LinDistFlow model):

$$
\min _{P_{i}, Q_{i}, V_{i}, Q_{g i}} \sum_{i=0}^{n-1} r_{i} \frac{P_{i}^{2}+Q_{i}^{2}}{V_{i}^{2}}
$$

s.t. for $i=0,1, \ldots n-1$ :

$$
\begin{gathered}
\bar{V} \geq V_{i} \geq \underline{V}, \\
Q_{g i} \leq \sqrt{S_{i}^{2}-P_{g i}^{2}}, \\
P_{i+1}=P_{i}-P_{L, i+1}+P_{g, i+1},
\end{gathered}
$$




$$
\begin{gathered}
Q_{i+1}=Q_{i}-Q_{L, i+1}+Q_{g, i+1} \\
V_{i+1}^{2}=V_{i}^{2}-2\left(r_{i} P_{i}+x_{i} Q_{i}\right) .
\end{gathered}
$$

The power flow equations for practical distribution systems will be slightly more complex because lateral branches can be extended at any node. To model the complete circuit, there will be a set of DistFlow equations for any branch circuit and a connection power flow at the branch-out node. To solve the distribution VVO problem in a distributed fashion, certain relaxation and decomposition techniques (e.g., dual decomposition, dual ascent, ADMM, consensus algorithm) must be applied so that the original optimization problem is converted into a sum of multiple subproblems, which each voltage control device can solve individually - perhaps with some inputs from other voltage-control devices requiring communications - and collectively reach the optimum after a few iterations. So, the convergence, the time/computation efforts to reach convergence, and the deviation from the global optimal value are important criteria for evaluating the performance of such distributed optimization methods.

Nonetheless, the team wants to explore complete model-free voltage control approaches (i.e., autonomous voltage-control functions without any communication) because this provides the most scalability and the least implementation cost for high-penetration solar PV scenarios. The team's objective is to investigate how well such approaches (i.e., global optimal vs. suboptimal) can directly or indirectly achieve the minimized objective function (e.g., total power loss or solar power curtailment/mechanical switching actions). This would improve understanding of the trade-offs of control vs. optimization and communication vs. optimality, as well as offer different operation choices for DSOs. For brevity, the summary of the techniques explored is given as follows.

1. Net-zero distribution node: If the local load real power demand connected to the node can follow with the local solar power generation and the solar PV inverter injects or absorbs the local measured load reactive power, then this distribution node will become net-zero and thereby minimize the distribution power flow and power loss accordingly. In practice, the exact net-zero might be difficult, but it is possible to offset partial power flow and contribute to the power loss minimization. Because there is no interaction between the primary feeder voltage control devices and PV inverters, the system will always be stable.

2. Piecewise linear voltage control (i.e., droop control or volt-var control: One common local voltage control characteristic is the piecewise linear voltage control. This control function has been included in IEEE 1547.8 (withdrawn) and reinstated in the newly published IEEE Standard 1547-2018. The voltage control with IEEE 1547 control curve was observed [47] to show instability in single PV inverter and multiple PV inverter cases in which reactive power injection and voltage exhibit undesired oscillatory behaviors between two operating points. Control delays, larger droop slope, and step size all contribute to this instability issue. The solutions that can mitigate this issue are:

- smooth by a first order low-pass filter,

- incremental voltage control, and

- smooth approximate control curve.

3. Incremental voltage control: The piecewise linear voltage control exhibits oscillatory (i.e., haunting) behavior, as previously discussed. This instability concern can be mitigated by inserting a first-order low-pass filter. Alternatively, it is possible to design a control algorithm so that the reactive power decision relies not only the bus voltage but also on previous reactive power injection, leading to the so-called incremental voltage control. This voltage control algorithm is similar to an integral controller and can effectively mitigate the voltage fluctuations. The decentralized voltage control was proposed in $\mathrm{Li}, \mathrm{Qu}$, and Dahleh [119]. Thus far, the incremental voltage control algorithm only 
addresses voltage regulation to maintain within acceptable ranges. In future work, we will revisit the incremental control algorithm and apply a sub-gradient method to minimize power loss.

4. Modified voltage control curve: As discussed in point 2, the piecewise linear voltage control curve has demonstrated convergence problems with the solution jumping back and forth across the points where the first derivative of voltage control curve is discontinuous. In Turitsyn et al. [48], this issue was eased by a modified voltage control curve, which is smoothed by using a sigmoid function, as shown in Eq. (A-7) and Figure A-36. In Eq. (A-7), the parameter $\delta$ can be varied to control how closely the smoothed curve approximates the sharp transition of the original control function.

$$
G\left(q_{j}^{\max }, V_{j}, \delta\right)=q_{j}^{\max }\left(1-\frac{2}{1+\exp \left[-4\left(V_{j}-1\right) / \delta\right]}\right)
$$

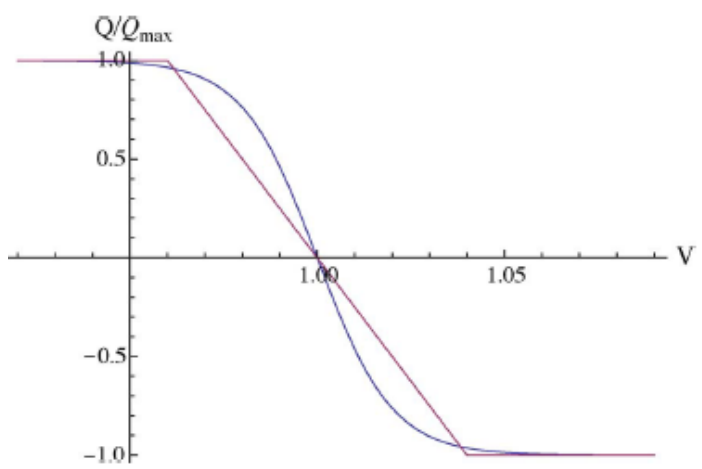

Figure A-36. Voltage control curve smoothed by a sigmoid function [48].

\section{A.4.3 Adaptive and/or Feedback Voltage Control}

The reactive power capability of a solar PV inverter is subjected to its designed apparent power capacity and limited by the real power output set by the MPPT controller. This is because PV inverters are primarily used for grid (i.e., energy) feeding with limited secondary or ancillary grid (voltage) supporting functions. At certain distribution nodes where a higher reactive power injection or absorption is needed, distribution STATCOM devices can be placed, assuming the responsibility of voltage regulation. In this case, closed-loop feedback voltage controls can be designed for voltage regulation by using regular PI controllers, self-tuning, or model-reference adaptive voltage regulators.

The distribution voltage and var control problem has essentially become a multi-timescale, multidevice coordination and optimization problem that involves utility-controlled devices and devices owned by independent power producers and consumers or prosumers. Completely centralized or completely distributed approaches might be impractical. With existing utility structure and various emerging demandside resources, a pragmatic and holistic approach would be helpful to address the distribution voltage control problem. First, the team considered the existing market structure and regulating devices in medium-voltage distribution grids. Energy efficiency was pursued by utilities in 1970s due to the oil embargo and was pursued once again in the early 2000s due to global warming; as a result, CVR programs rose in popularity. It was not until the 2010 s that utilities started to shift their strategies toward VVO programs as part of smart grid demonstration projects.

Opposite from the demand side, DERs such as solar PV inverters have experienced a series of control function changes per standard requirements, mainly IEEE Standard 1547. Initially, solar PV inverters followed the operation strategy of purely energy or grid feeding (i.e., MPPT and current control modes) 
and deenergize the grid (e.g., abnormal grid voltage and frequency conditions, grid faults) based on previous standard requirements specified in IEEE 1547-2003. With the increasing penetration of solar power and corresponding utility operation challenges, such as the duck curve phenomena, utilities have changed their attitude toward PV inverters from ignoring them to regarding them as assets that can aid in grid management during shortages. This has pushed the addendum (IEEE 1547a-2014) and the full revision approved as IEEE 1547-2018. Nonetheless, IEEE 1547 only defines the interconnection requirements of DERs but does not fully addresses the active operation and control coordination of DERs after the interconnection. Generally, these efforts of defining autonomous control functions are still at the initial phase in which the team called local functions and the operations of utility-controlled devices (e.g., LTCs, voltage regulators, capacitor banks, STATCOM) and solar PV inverters are still isolated. So, the next phase would be coordinated controls to ensure that control efforts from various devices are not contradicted. The last phase would be to achieve a unified and globally optimized control among all the control devices and resources, including load control, within each specific distribution feeder or system. As a logical step, the team will follow this bottom-up integration strategy from the local level to the coordinated level to the optimized level, whereas the controls of multiple devices are coordinated locally at each level, thereby contributing to the overall optimization goal of energy efficiency.

At the local level, the solar PV inverters and responsive loads at each distribution feeder node are designed to follow each other (i.e., the HVAC load control following solar PV active power production and the solar PV inverter following load reactive power), which subjectively reduces solar power curtailment and line power loss because of the required power flows short distance and voltage regulation requirements provided by utility-controlled devices.

At the coordinated level, devices at different timescales must be coordinated in certain ways for efficient operation. For the utility-controlled devices - mainly LTCs at distribution substation, voltage regulators, and capacitor banks - the strategy is to track slow voltage variations that follow daily load consumption patterns (i.e., base voltage regulation) and unexpected contingencies. Their hourly voltage references are set up 1 day ahead on the basis of utility VVO and forecasted load profiles. This will limit the voltagecontrol range for DERs so that these nonutility control devices can assume short time or fast voltage variations and regulation services locally in a fast way and thus the utility-controlled devices would not observe the voltage fluctuations around their voltage reference points, reducing their tap operations or switching actions and extending their lifetime. The utility load forecasting would have a learning curve to adapt to the net load profiles under DER controls over the time. For nonutility dispersed devices, such as solar PV inverters, the voltage references can be obtained from a short timescale (e.g., 1-5 min), modelbased feeder power flow analysis or by consensus algorithm, which takes the average among neighboring nodes. The 5 min adaptive load control method can mitigate voltage ramping variations due to passing clouds at midday, ramping up in the morning and ramping down in the afternoon. PV inverters can be deployed to control fast voltage variations at tens of seconds so that these voltage fluctuations will not activate LTC and SVR due to their time delay setting typically around $60 \mathrm{~s}$. Figure A-37 illustrates the three timescales' voltage control coordination. The purpose of coordination is to ensure that the system is operating properly within the permissible voltage limits as specified by ANSI and that each device is contributing voltage regulation based on its own capability.

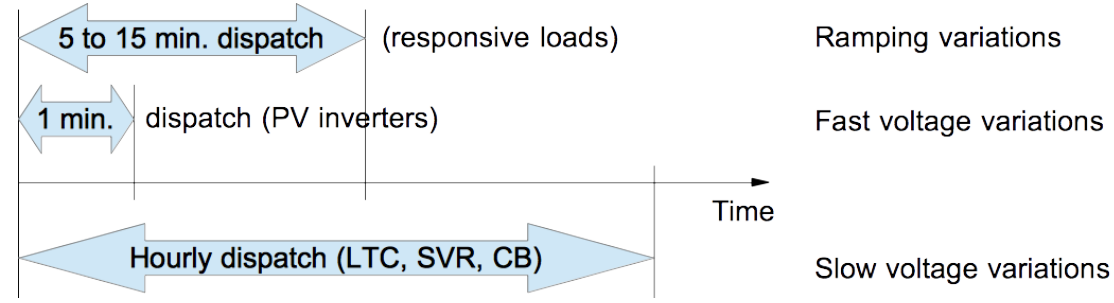

Figure A-37. Voltage control coordination at different timescales. 
The ultimate level is the optimized level in which the system is working at the best economical and efficient state - which has minimal overall power loss, renewable power curtailment, and control effortwhile still maintaining power quality and safety and serving customer requirements. This will require finding the best voltage and reactive power references for each device in a timely manner via advanced distributed optimization techniques. Distributed optimization will involve the processes of problem formulation (i.e., defining objective functions to be optimized and constraints to be satisfied), problem decomposition, information discovery, and iterative algorithms. Decomposition is necessary for solving a problem by dividing it into smaller problems and solving each separately, either in parallel or sequentially. There are generally two decomposition techniques: basic (i.e., primal) decomposition and dual decomposition. An information discovery scheme defines a communication map for who is communicating with whom, what information is shared, and how it is used, synchronized, or asynchronized. Figure A-38 shows an example map for distribution feeder nodes. Finally, distributed computation methods are used to update the decision variables (e.g., active power, reactive power, voltage, Lagrange multipliers) individually at each device toward the optimum through iterations. At this time, the team chose the ADMM, which converges significantly faster than other algorithms.

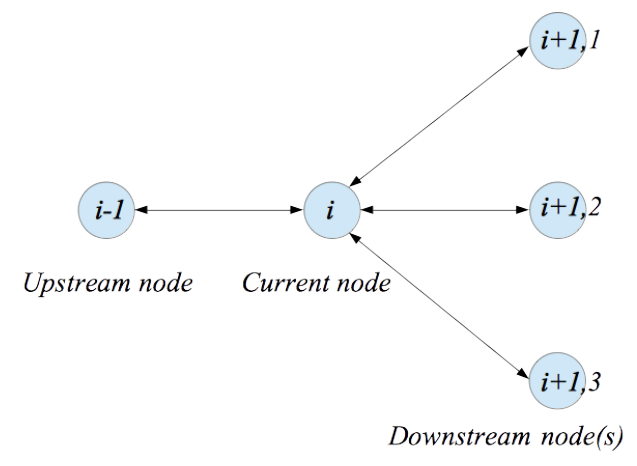

Figure A-38. Information discovery scheme.

The team explored different local voltage control strategies and discussed related stability issues. The team's motivation was to devise a new feedback dynamic (i.e., iterative) control mechanism on the basis of local voltage control so that it can be treated as a distributed algorithm for solving a convex global optimization problem (e.g., minimizing total circuit power loss). The context of distributed optimization and control relative to the centralized optimization problem and the local control problem are illustrated in Figure A-39. In a centralized optimization problem, one must model the process and variables in the objective functions and constraints in an offline manner, whereas in a control problem, the real-world process does not necessarily need to be modeled, and the feedback approach is used to drive the process variable toward a desired state. The team's motivation was to develop online and model-free optimization methods by using feedback and iterative algorithms. With this focus in mind, the following topics and work are continuing to be conducted.

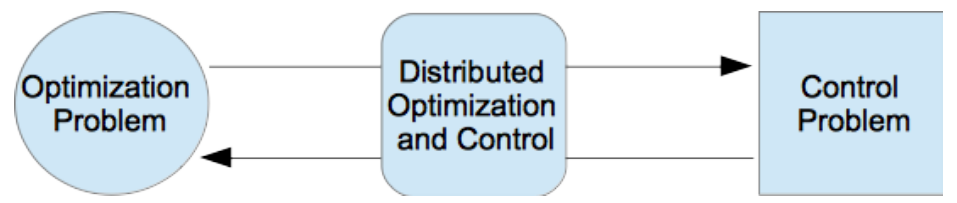

Figure A-39. Context of distributed optimization and control problem. 
Evaluation of control vs. optimization and communication vs. optimality: As discussed previously, distribution voltage regulation and loss minimization are conflicting objectives. There is a need to further study the trade-off of stability vs. optimality. Additionally, the role of communication in optimization shall be investigated to understand the trade-off of communication vs. optimality. Understanding these two topics will help the team design a more appropriate secondary control strategy to achieve the interested objectives.

Communication vs. optimality: There does seem to be a question regarding the role of communications on global optimization, just as a centralized controller relies on communications and can achieve global optimality. The question asks whether multiple distributed controllers achieve global optimality without any direct and explicit communication to each other or to what extent (i.e., minimum communication requirements on the data quantity and communication bandwidth) global optimality can be achieved. On DVC, there are a few discussions regarding this topic. The value of communication in the voltage regulation problem was investigated in Stitt [2] to analyze the performance of purely local voltage regulation strategies and the distributed control strategies where minimal communications are allowed. The analysis and IEEE 123 bus test feeder simulation proposed agent-to-agent communication plays a fundamental role in the problem of DVC in terms of voltage regulation performance. However, the authors have not investigated the performance of minimizing total power loss. Bolognani, Cavraro, and Zampieri [120] compared the distribution power loss performance of distributed algorithms with complete communication graph and two different edge-disjoint communication graphs (uniform activation probability vs. optimal activation probability for each edge). The results showed that it was possible to achieve less power loss by using optimized communication strategy instead of by using complete communication graph. Although it is possible to develop a communication-free algorithm with locally available information to achieve a globally optimal solution, as reported in Zhang and Zhu [121], by using game-theoretic approaches, the team pursued a distributed algorithm with either scalable neighboring communications or implicit "communication" through feedback and iteration approach.

Control vs. optimization: Generally, control problems deal more with stability issues on a real-time basis, whereas optimization problems provide an optimal setting in an offline manner during planning. With emerging distributed optimization techniques, there are interests [122-125] to interpret a centralized or distributed convex optimization problem from control perspective, and vice versa, to interpret a feedback dynamical system as a distributed algorithm for solving a convex global optimization problem. This effort has shed light on the control-theoretic or feedback approach for optimization problems and could achieve the goal with less computation power in the controller and without solving any power flow model (i.e., model-free). Limited communication (e.g., among neighboring nodes) might be necessary for agent coordination.

Design of DVC secondary control strategy: A review of DVC methods can be found in Satsangi and Kumbhar [106] and Luo et al. [107] in which different optimization problem formulations and various iterative algorithms for distributed optimization were adopted. In this work, the team focused on radial distribution systems under high-penetration solar PV scenarios in which smart PV inverters and load controllers provide system functions of coordinated voltage control and system loss optimization to a certain extent if not in a global sense, which is desired. This concept of "smart inverter with integrated system functions" was originally proposed in the team's previous work [126] and has not yet been fully investigated. The team wants to pursue this direction for the aforementioned reasons: scalable, modelfree, and minimal communication requirements.

\section{A.4.4 Problem Formulation}

First, a global optimization problem formulation will be constructed. The objective functions can be to minimize the system's real power losses, the total voltage deviations of all the nodes (usually in quadratic 
form because it fits well into most established optimization methods), the total cost of reactive power generation from all the controllable devices, or a weighted combination of these three objectives. In this problem, the team considered total system loss solely as the objective function. For voltage regulation, a flat voltage profile might be elegant but might also be inefficient from a system perspective. Therefore, a flexible voltage profile is necessary if the voltage constraints are placed for all the nodes based on ANSI limits. The cost aspect of voltage control and reactive power compensation can be associated with gametheoretic approaches, which are handled as a separate optimization problem.

There are two ways to model the system power loss: directly and indirectly. The direct method models the line loss at $\mathrm{I}^{2} \mathrm{R}$ with explicit line resistance parameters, whereas the indirect method applies power balance and calculates the system loss as the balance of generation and load. Accordingly, system loss can be computed by the sum of the loss at each node (indirect) or the direct line loss, including transformer loss. The direct method is only accurate when all the component losses are modeled.

Direct system loss modeling: The DistFlow branch model is used to formulate the direct system power loss problem:

$$
\min _{P_{i}, Q_{i}, V_{i}, Q_{g i}} \sum_{i=0}^{n-1} r_{i} \frac{P_{i}^{2}+Q_{i}^{2}}{V_{i}^{2}}
$$

s.t. for $i=0,1, \ldots n-1$ :

$$
\begin{gathered}
P_{i+1}=P_{i}-r_{i} \frac{P_{i}^{2}+Q_{i}^{2}}{V_{i}^{2}}-P_{L, i+1}+P_{g, i+1}, \\
Q_{i+1}=Q_{i}-x_{i} \frac{P_{i}^{2}+Q_{i}^{2}}{V_{i}^{2}}-Q_{L, i+1}+Q_{g, i+1}, \\
V_{i+1}^{2}=V_{i}^{2}-2\left(r_{i} P_{i}+x_{i} Q_{i}\right)-\left(r_{i}^{2}+x_{i}^{2}\right) \frac{P_{i}^{2}+Q_{i}^{2}}{V_{i}^{2}}, \\
\bar{V} \geq V_{i} \geq \underline{V}, \\
Q_{g i} \leq \sqrt{S_{i}^{2}-P_{g i}^{2}} .
\end{gathered}
$$

Model-free or indirect loss modeling: In this work, the teal modeled the system losses indirectly, and the distribution feeder topological information (i.e., locations of distributed energy resources and loads, as well as how they are connected) will be modeled in the power flow equations. Because the reactive current contributes to the line loss, the team considered minimizing real power losses and "reactive power losses," which are essentially the reactive power exchange between line reactance and reactive power sources. The quadratic form of the system losses was also used here:

$$
\min _{Q_{g i}} \quad\left(\sum_{i=0}^{n-1} P_{g i}-\sum_{i=0}^{n-1} P_{d i}\right)^{2}+\left(\sum_{i=0}^{n-1} Q_{g i}-\sum_{i=0}^{n-1} Q_{d i}\right)^{2}
$$

The team assumed that the load demands are noncontrolled parameters or given parameters by other control mechanisms, and none of the DER real power generation participates in the voltage-control problem and system loss optimization because of other objectives (e.g., maximizing renewable energy). Therefore, the team only minimized the reactive power portion of system losses: 


$$
\min _{Q_{g i}} \quad\left(\sum_{i=0}^{n-1} Q_{g i}-\sum_{i=0}^{n-1} Q_{d i}\right)^{2}
$$

In distribution systems, there are five different node configurations:

- load-only node (i.e., $P_{g i}=0$ and $Q_{g i}=0$ ),

- $\quad$ DG-only node (i.e., $P_{d i}=0$ and $Q_{d i}=0$ ),

- $\quad$ load with DG node (i.e., $P_{g i} \neq 0, Q_{g i} \neq 0, P_{d i} \neq 0$, and $Q_{d i} \neq 0$ ),

- $\quad$ purely branch node (i.e., $P_{g i}=0$, $Q_{g i}=0, P_{d i}=0$, and $Q_{d i}=0$ ), and

- $\quad$ substation bus node (i.e., $P_{g i} \neq 0, Q_{g i} \neq 0$ ) but uncontrolled from the distribution side or in this problem; essentially, they can be treated as infinity, and the inbound power flow will balance the difference of generation and load within the target distribution system, which will be minimized in this problem.

The voltage and reactive power generation constraints shown by Eqs. (A-12)-(A-13) must be included. The remaining constraints are the topological power flow models and power balance equations, which the team will substitute with a simulation based-model and direct power flow and voltage measurements. This brings the benefit of simple computation and fast convergence to integrate distributed optimization more tightly with local controls.

Problem decomposition: Decomposition is a general approach for solving a problem by dividing it into smaller problems and solving each separately, either in parallel or sequentially. There are generally two decomposition techniques: basic (primal) decomposition and dual decomposition. This will be investigated in future work.

Iteration algorithms and information discovery: Distributed optimization relies on iterative algorithms to update the decision variables toward the optimum. Information from other neighboring distributed devices or agents are most likely required, so an information discovery scheme must be designed. The team will investigate the distributed computation methods, such as the ADMM, which is known to converge significantly faster than other algorithms. Consensus algorithms will be applied for information discovery.

Development of a co-simulation platform for performance evaluation: The convergence, time and computation efforts to reach convergence, and deviation from the global optimal value are important criteria for evaluating the performance of distributed optimization methods. A simulation platform is required for control and optimization performance evaluation. For distribution systems that adopt DVC strategies, the team is developing a MATLAB/OpenDSS co-simulation platform. The distribution feeder circuit and components are modeled by using OpenDSS scripts. The DVC control algorithms are implemented in the MATLAB environment. The MATLAB codes will call for the OpenDSS power flow solver, which uses an open-source sparse matrix solver ("KLUSolve") for power flow analysis. This power flow analysis will be performed in a controlled snapshot mode with embedded adaptive load control and DVC for smart inverters. The framework of a multi-timescale co-simulation platform, as shown in Figure A-40, comprises five modules: (1) a long timescale simulation tool to model power system dynamics daily, weekly, or yearly with minutes time steps; 2) a short timescale simulation tool to model the detailed power electronics dynamics at seconds with microseconds time steps; (3) multitimescale control modeling (e.g., 5 min PV inverter control, 15 min load control, and hourly LTC control); (4) an optional monitoring and governing module to enable the detailed converter modeling when potential quality and instability issues identified; and (5) the interface module to link all the 
modules. An experimental setup of the planned co-simulation platform was implemented with OpenDSS, MATLAB, and Simulink/Simscape/Electronics, as shown in Figure A-41. The power network partition concept is shown in Figure A-42 in which the data exchange and conversion between phasor-based modeling for the power system and the waveform simulation for power electronics is performed in the aforementioned interfacing module.

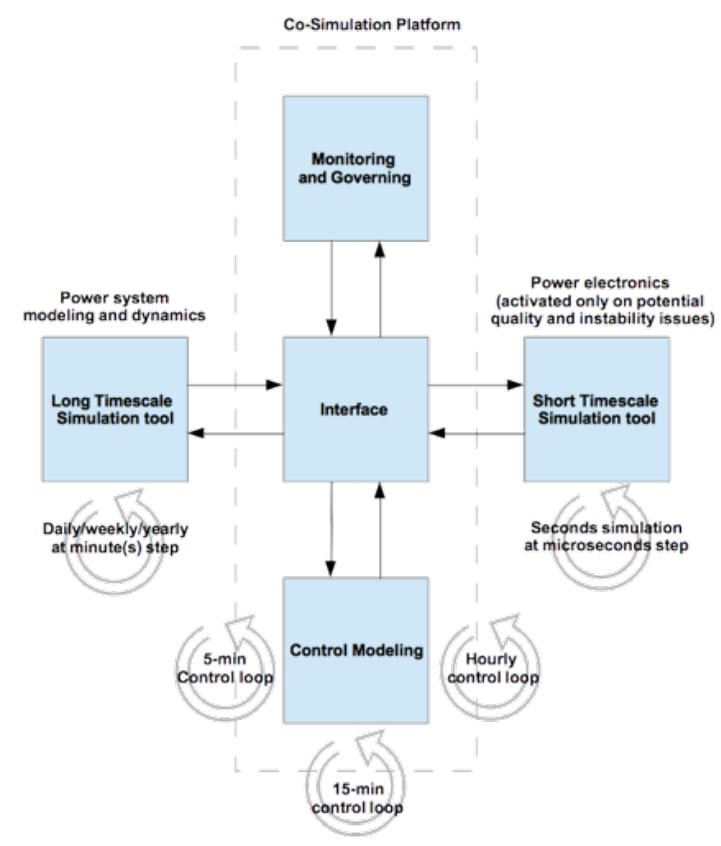

Figure A-40. Framework of proposed multi-timescale co-simulation platform.

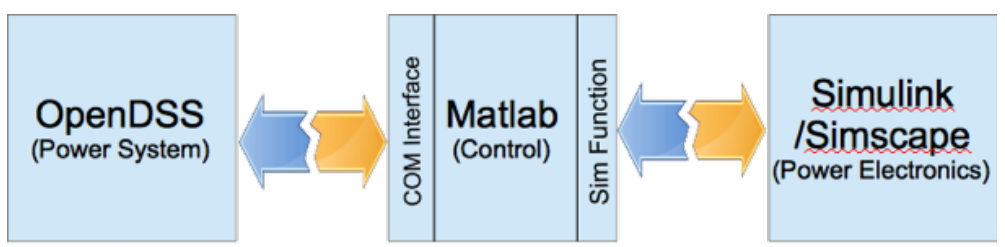

Figure A-41. An experimental implementation of the co-simulation platform. 


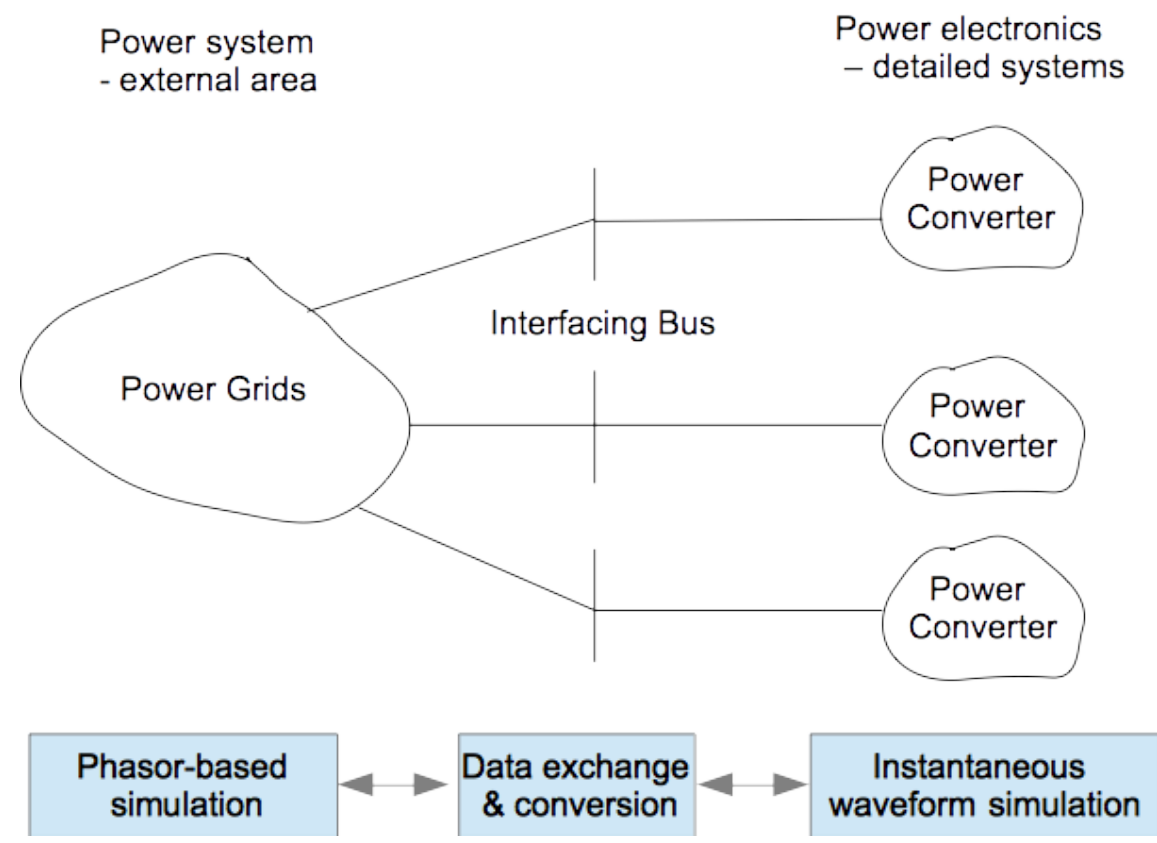

Figure A-42. Power network partition for co-simulation.

\section{A.4.5 Accelerating DVC}

This section discusses the acceleration of solving PV inverter distributed optimal control by using modified ADMM and discusses coordination between the optimal PV control and smart building control.

The distributed optimization model for PV control is formulated based on the SOCP-based branch flow model [54-55] of distribution networks. The objectives are to minimize the total nodal voltage deviation and network power loss.

$$
f=\min \left(\varpi_{1} \xi \sum_{j=1}^{n}\left(u_{j}^{+}-2 v_{j}^{+} v_{N j}^{+}+\left(v_{N j}^{+}\right)^{2}\right)+\varpi_{2} \sum_{j=1}^{n} r_{j-1} l_{j}^{-}\right) .
$$

s.t. $\forall j=1, \cdots, n$ :

$$
\begin{gathered}
\varpi_{1}+\varpi_{2}=1, \\
\left\|\left[\begin{array}{lll}
2 P_{j}^{-} & 2 Q_{j}^{-} & l_{j}^{-}-u_{j}^{-}
\end{array}\right]\right\|_{2} \leq l_{j}^{-}+u_{j}^{-}, \\
\left\{\begin{array}{l}
P_{j}^{-}-P_{j}^{+}-l_{j}^{-} r_{j-1}-p_{j}^{(c)}+p_{j}^{(g)}=0 \\
Q_{j}^{-}-Q_{j}^{+}-l_{j}^{-} x_{j-1}-q_{j}^{(c)}+q_{j}^{(g)}=0
\end{array}\right. \\
u_{j}^{+}=u_{j}^{-}-2\left(r_{j-1} P_{j}^{-}+x_{j-1} Q_{j}^{-}\right)+\left(r_{j-1}^{2}+x_{j-1}^{2}\right) l_{j}^{-},
\end{gathered}
$$




$$
\begin{aligned}
& P_{j}^{+}=P_{j}, P_{j}^{-}=P_{j-1}, P_{n}=0 \\
& Q_{j}^{+}=Q_{j}, Q_{j}^{-}=Q_{j-1}, Q_{n}=0 \text {, } \\
& \left\|\left[\begin{array}{ll}
2 v_{j}^{+} & u_{j}^{+}-v_{N j}^{+}
\end{array}\right]\right\|_{2} \leq u_{j}^{+}+v_{N j}^{+}, \\
& \left(v_{j}^{\min }\right)^{2} \leq u_{j}^{+} \leq\left(v_{j}^{\max }\right)^{2}, \\
& u_{j}^{+}=u_{j}, u_{j}^{-}=u_{j-1}, u_{1}^{-}=1 \\
& \left\|\left[p_{j}^{(g)} \quad q_{j}^{(g)}\right]\right\| \|_{2} \leq s_{j}, \\
& 0 \leq p_{j}^{(g)} \leq p_{j}^{\max } \\
& \left\{\begin{array}{l}
P_{G, 0}^{\min } \leq P_{G, 0} \leq P_{G, 0}^{\max } \\
Q_{G, 0}^{\min } \leq Q_{G, 0} \leq Q_{G, 0}^{\max } \\
v_{0}=1.0 \text { p.u. }
\end{array},\right.
\end{aligned}
$$

where $u_{i}$ is the square of the voltage of node $i ; l_{i j}$ is the square of the current in line $i j ; u_{0}$ is the square of nominal voltage, which is $1.0 \mathrm{p} . \mathrm{u}$; $v_{i}$ is the nodal voltage; $P_{i j}$ and $Q_{i j}$ are the active and reactive power in line $i j ; P_{G, i}$ and $Q_{G, i}$ are the active and reactive power injections at the substation node; $S_{G, i}$ is the capacity of the substation; $S_{P V, j}, P_{P V, j}$, and $Q_{P V, j}$ are the capacity, active, and reactive power output, respectively, of the PV at node $j ; P_{D, j}$ and $Q_{D, j}$ are the active and reactive power demand at node $j . u+j, u-j$; and $P+j, P_{-j}$, $Q+j$, and $Q-j$ are the local variables corresponding to $u_{j}, u_{j-1}, P_{j}, P_{j-1}, Q_{j}$ and $Q_{j-1}$ at node $j$.

The ADMM-based algorithm is solved as follows. First, an augmented Lagrangian function of each local optimization problem is constructed for each PV inverter, as shown in Eq. (A-28):

$$
\mathrm{L}^{\mathrm{ADMM}}=\sum_{j=1}^{n} \mathrm{~L}_{j}^{\mathrm{ADMM}},
$$

where:

$$
\begin{aligned}
\mathrm{L}_{j}^{\mathrm{ADMM}}= & {\left[\varpi_{1} \xi\left(u_{j}^{+}-2 v_{j}^{+} v_{N j}^{+}+\left(v_{N j}^{+}\right)^{2}\right)+\varpi_{2}\left(r_{j-1} l_{j}^{-}\right)\right] } \\
& +\frac{\rho}{2}\left(P_{j}^{+}-P_{j}\right)^{2}+\frac{\rho}{2}\left(P_{j}^{-}-P_{j-1}\right)^{2}+\frac{\rho}{2}\left(Q_{j}^{+}-Q_{j}\right)^{2}+\frac{\rho}{2}\left(Q_{j}^{-}-Q_{j-1}\right)^{2} \\
& +\frac{\rho}{2}\left(u_{j}^{+}-u_{j}\right)^{2}+\frac{\rho}{2}\left(u_{j}^{-}-u_{j-1}\right)^{2}+\lambda_{j}^{P^{+}}\left(P_{j}^{+}-P_{j}\right)+\lambda_{j}^{P^{-}}\left(P_{j}^{-}-P_{j-1}\right) \\
& +\lambda_{j}^{Q^{+}}\left(Q_{j}^{+}-Q_{j}\right)+\lambda_{j}^{Q^{-}}\left(Q_{j}^{-}-Q_{j-1}\right)+\lambda_{j}^{u^{+}}\left(u_{j}^{+}-u_{j}\right)+\lambda_{j}^{u^{-}}\left(u_{j}^{-}-u_{j-1}\right) .
\end{aligned}
$$


Then, for each iteration, the following steps are performed.

Solve local optimization problem: for node $j$,

$$
\min _{P_{j}^{-}, P_{j}^{+}, Q_{j}^{-}, Q_{j}^{+}, u_{j}^{+}, u_{j}^{-}, z_{j}} \mathrm{~L}_{j}^{\mathrm{ADMM}} .
$$

The solution is marked as $P+j(k+1), P-j(k+1), Q+j(k+1), Q-j(k+1), u+j(k+1)$, and $u-j(k+1)$.

Update global variables $P, Q$, and $u$ :

$$
\begin{gathered}
\forall j=1, \ldots, n-1: \\
P_{j}(k+1)=\frac{1}{2}\left(P_{j}^{+}(k+1)+P_{j+1}^{-}(k+1)\right) \\
Q_{j}(k+1)=\frac{1}{2}\left(Q_{j}^{+}(k+1)+Q_{j+1}^{-}(k+1)\right) \\
u_{j}(k+1)=\frac{1}{2}\left(u_{j}^{+}(k+1)+u_{j+1}^{-}(k+1)\right) \\
P_{n}(k+1)=0, P_{0}(k+1)=P_{1}^{-}(k+1), \\
Q_{n}(k+1)=0, Q_{0}(k+1)=Q_{1}^{-}(k+1), u_{n}=u_{n}^{+} .
\end{gathered}
$$

Update Lagrangian multipliers:

$$
\begin{aligned}
& \lambda_{j}^{P^{+}}(k+1)=\lambda_{j}^{P^{+}}(k)+\rho\left(P_{j}^{+}(k+1)-P_{j}(k+1)\right) \\
& \lambda_{j}^{P-}(k+1)=\lambda_{j}^{P^{-}}(k)+\rho\left(P_{j}^{-}(k+1)-P_{j-1}(k+1)\right) \\
& \lambda_{j}^{Q^{+}}(k+1)=\lambda_{j}^{Q^{+}}(k)+\rho\left(Q_{j}^{+}(k+1)-Q_{j}(k+1)\right) \\
& \lambda_{j}^{Q-}(k+1)=\lambda_{j}^{Q^{-}}(k)+\rho\left(Q_{j}^{-}(k+1)-Q_{j-1}(k+1)\right) \\
& \lambda_{j}^{u^{+}}(k+1)=\lambda_{j}^{u^{+}}(k)+\rho\left(u_{j}^{+}(k+1)-u_{j}(k+1)\right) \\
& \lambda_{j}^{u^{-}}(k+1)=\lambda_{j}^{u^{-}}(k)+\rho\left(u_{j}^{-}(k+1)-u_{j-1}(k+1)\right) .
\end{aligned}
$$

When the primal and dual residuals converge to the preset thresholds or the number of iterations reaches its maximum, the solving process stops.

To accelerate the convergence and solving process, the team applied an over-relaxed ADMM algorithm and an adaptive penalty parameter method to solve the distributed optimal PV control problem faster and meet the requirements of practical applications. 


\section{Over-relaxed ADMM:}

The over-relaxed ADMM is a variant of ADMM [127-128], which introduces a relaxation parameter $\alpha \in$ $(0,2)$ and replaces $A x^{k+1}$ with $\alpha A x^{k+1}-(1-\alpha)\left(B z^{k}-c\right)$ in the update of $z$ and $\lambda$. For a general optimization problem:

$$
\begin{array}{cc}
\underset{x \in \mathrm{X}, x \in Z}{\operatorname{minimize}} & f(x)+g(z) \\
\text { subject to } & A x+B z=c
\end{array}
$$

The augmented Lagrangian function is:

$$
\begin{aligned}
L_{\rho}(x, z, \lambda):= & f(x)+g(z)+\lambda^{T}(A x+B z-c) \\
& +\frac{\rho}{2}\|A x+B z-c\|^{2}
\end{aligned}
$$

The update process of ADMM is:

$$
\begin{aligned}
& x^{k+1} \in \underset{x \in \mathrm{X}}{\arg \min } L_{\rho}\left(x, z^{k}, \lambda^{k}\right) \\
& z^{k+1} \in \underset{z \in Z}{\arg \min } L_{\rho}\left(x^{k+1}, z, \lambda^{k}\right) \\
& \lambda^{k+1}=\lambda^{k}+\rho\left(A x^{k+1}+B z^{k+1}-c\right)
\end{aligned} .
$$

The update process of over-relaxed ADMM is:

$$
\begin{aligned}
& x^{k+1} \in \underset{x \in \mathrm{X}}{\arg \min } L_{\rho}\left(x, z^{k}, \lambda^{k}\right) \\
& \hat{\lambda}^{k+1}=\lambda^{k}+\rho(\alpha-1)\left(A x^{k+1}+B z^{k}-c\right) \\
& z^{k+1} \in \underset{z \in Z}{\arg \min } L_{\rho}\left(x^{k+1}, z, \hat{\lambda}^{k+1}\right) \\
& \lambda^{k+1}=\hat{\lambda}^{k+1}+\rho\left(A x^{k+1}+B z^{k+1}-c\right)
\end{aligned}
$$

Compared with the general ADMM, Eqs. (A-35)-(A-36) show that the over-relaxed ADMM adds an additional step of calculating $\hat{\lambda}^{k+1}$ and using it to update $z$ and $\lambda$. When $\alpha=1.0$, Eqs. (A-35)-(A-36) are equivalent. In this case, the over-relaxed ADMM algorithm achieved good performance when $\alpha \in[1.5,1.8]$. The steps for the over-relaxed ADMM algorithm are described as follows. 


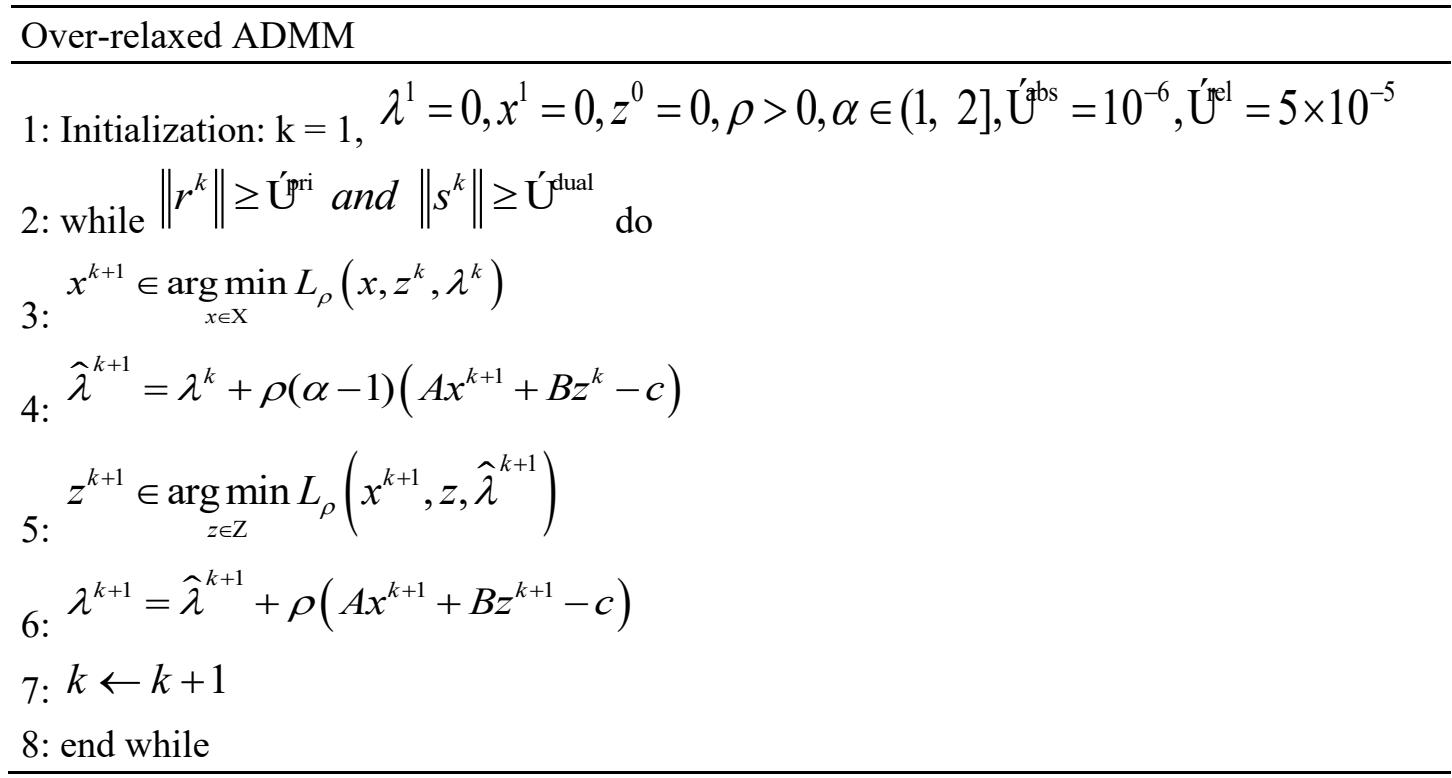

\section{Adaptive penalty parameter:}

The primal and dual residuals have different convergence speeds, which impacts the convergence of the overall solving process. The convergence of the two residuals can be coordinated or balanced by adjusting the penalty parameter $\rho$. When the penalty factor increases, the primal residual will decrease, but the dual residual will increase and vice versa. Therefore, the team applied an adaptive penalty parameter method [129-130] to further accelerate the convergence. Equation (62) was used to balance the primal and dual residual magnitudes to be within a factor of $\mu$ of one another as they both converge to zero.

$$
\begin{array}{ll}
\rho^{k+1}= \begin{cases}\rho^{k}+\tau^{\text {incr }} & ,\left\|r^{k+1}\right\|_{2}>\mu^{\text {incr }}\left\|s^{k+1}\right\|_{2} \\
\rho^{k}-\tau^{\text {decr }} & ,\left\|s^{k+1}\right\|_{2}>\mu^{\text {decr }}\left\|r^{k+1}\right\|_{2} \\
\rho^{k} & , \text { Other }\end{cases} \\
\rho^{k+1} \geq 1
\end{array}
$$

where $\mu^{\text {incr }}, \mu^{\text {decr }}, \tau^{\text {incr }}$, and $\tau^{\text {decr }}$ are parameters, which are set to 6 and 4 , respectively. $\rho^{k+1}$ is a penalty parameter associated with each consensus constraint. $r^{k+1}$ and $s^{k+1}$ are the local primal and dual residuals, respectively.

\section{Performance of the modified ADMM:}

The performance of the over-relaxed ADMM and adaptive parameter penalty method is demonstrated by comparing the general ADMM and modified ADMM algorithm. Figure A-43 shows that both methods have the same objective function value but that the modified ADMM takes fewer iterations to converge. The detailed comparison results are shown in Table A-3 in which the modified ADMM method is demonstrated to be more efficient in achieving faster convergence without losing any optimality. 


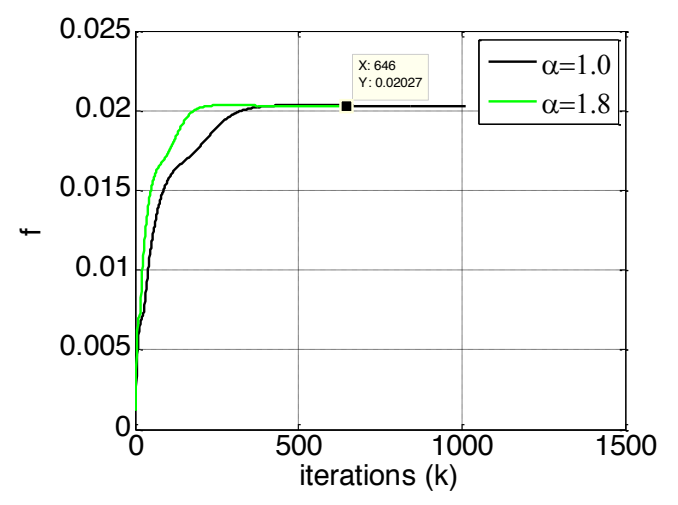

Figure A-43. Convergence of the objective functions. $\alpha=1.0$ is the general ADMM, and $\alpha=1.8$ is the modified ADMM.

Table A-3. Comparison results of the two methods.

\begin{tabular}{lcc}
\hline & $\alpha=1.0$ & $\alpha=1.8$ \\
\hline Number of iterations & 1,010 & 646 \\
Run time & $492.063 \mathrm{~s}$ & $310.233 \mathrm{~s}$ \\
\hline
\end{tabular}

The building control and PV control are at different timescales. The building loads, such HVAC units, are usually optimized and controlled for on/off status at 10 min time steps. The PV inverter can be controlled every minute or less. To mimic the online applications, the proposed distributed algorithm runs every minute to control the PV inverters for voltage regulation and loss reduction, and at every $10 \mathrm{~min}$, the team ran the optimal building control model, which is based on previous work [131]. The control logic is shown in Figure A-44. The building control aims to optimally control the on/off status of the HVAC units to track the variations of PV generations while meeting the temperature and comfort constraints. At each 10 min time step, the building control model optimizes and changes the shape of the total load curve at each node. Then, for every minute, the distributed optimal PV control algorithm optimizes the active and reactive power of each PV inverter for real-time voltage regulation and OPF. The optimization results are passed to OpenDSS [53] for simulation.

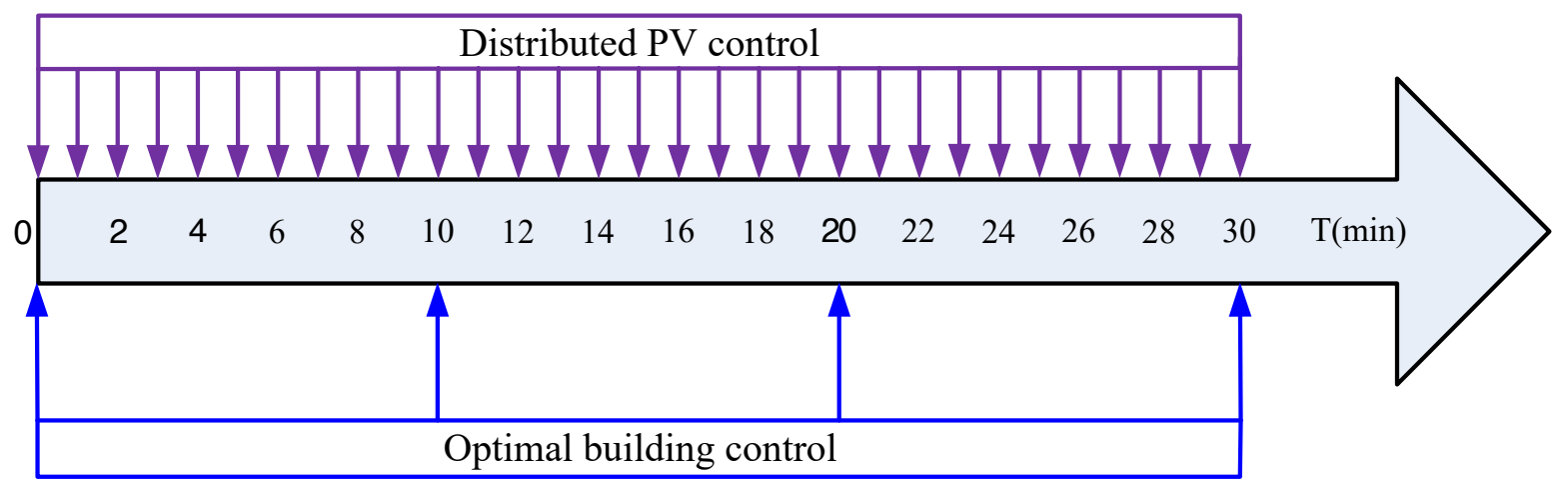

Figure A-44. The coordination between distributed optimal PV control and building control in distribution networks.

Then, the team wanted to validate the effectiveness of the optimization model and the modified ADMM algorithm in optimal PV control by comparing them with OpenDSS simulations. The proposed model was implemented in MATLAB and solved using CPLEX [57] on a personal computer. A modified IEEE 33 
node case [58] with 32 PVs were used for case studies. Second, the team conducted a comparative study to demonstrate the advantage of the proposed distributed optimal PV control over the traditional constant PF control. Third, the team conducted a comparative study to demonstrate the benefits of coordinating the optimal PV control and building control through time series simulations.

\section{Test Case:}

As shown in Figure A-45, there are 32 PVs in the IEEE 33 node testing system. The PV inverter capacity is $92 \mathrm{kVA}$. The team selected 11:00-15:00 as the study period and conducted distributed optimization for PV control for every minute. The PV generations and load curve are shown in Figures A-46 and A-47, respectively. As shown in Figure A-46, at 11:40, there was a significant drop of PV generations due to the cloud movement.

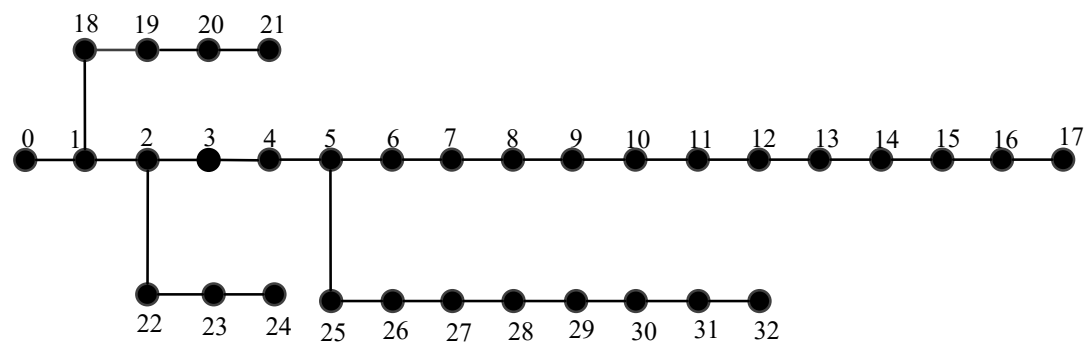

Figure A-45. IEEE 33 node testing system.

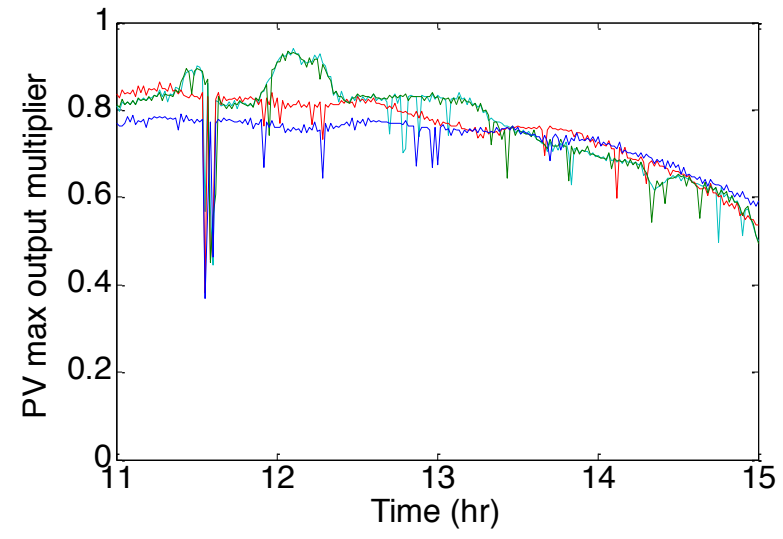

Figure A-46. Multipliers of PV generations.

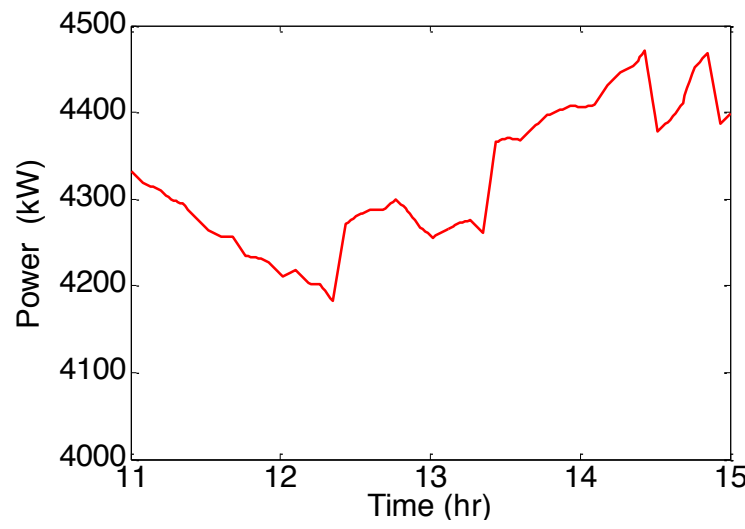

Figure A-47. Load curve.

\section{Comparison with OpenDSS simulation results:}

The results of the proposed distribution optimization model and modified ADMM algorithm were compared with those of the OpenDSS simulation to validate effectiveness and accuracy. The comparison results of nodal voltage and active/reactive power flow are shown in Figure A-48. In these figures, Vo is the result of OpenDSS, and Vd is the result of ADMM. The average errors were as follows: active power line flow was $0.5 \%$, reactive power line flow was $1.17 \%$, and nodal voltage was $2.9519 \mathrm{e}^{-3} \%$. The proposed model and algorithm cannot be concluded to be effective and accurate in distribution system optimization. 


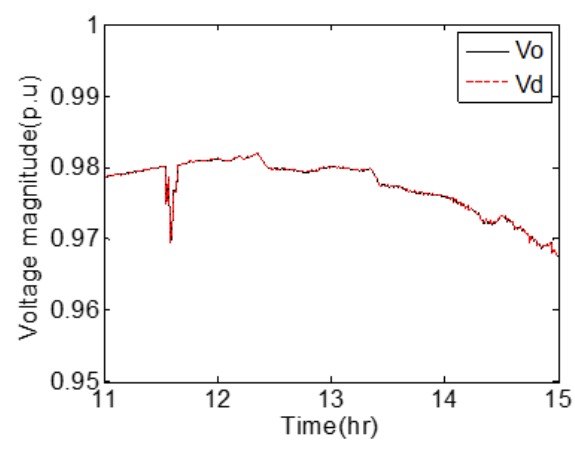

a) Voltage at node 18

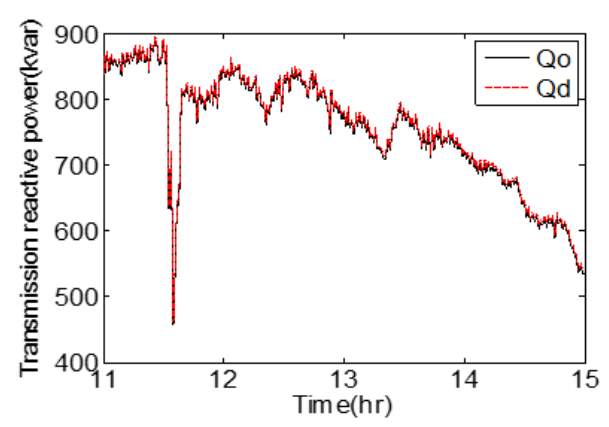

c) Reactive power flow in line 1-2

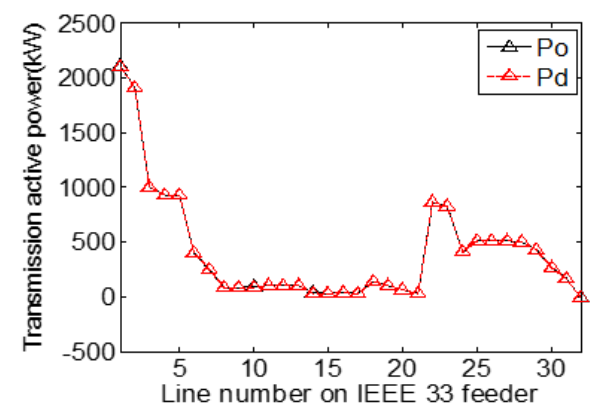

e) Active power flow at 13:00

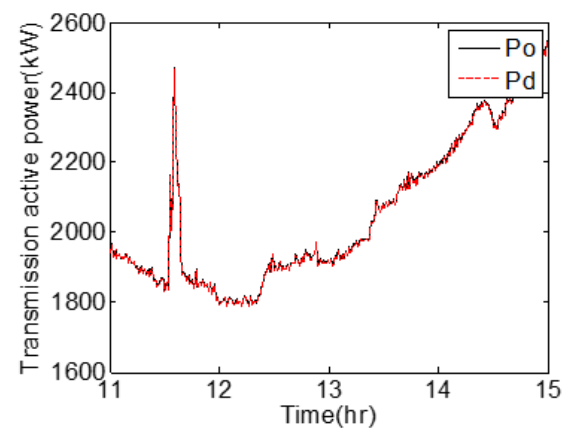

b) Active power flow in line 1-2

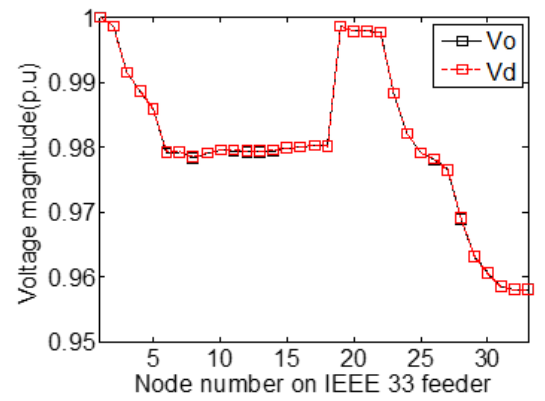

d) Nodal voltage at 13:00

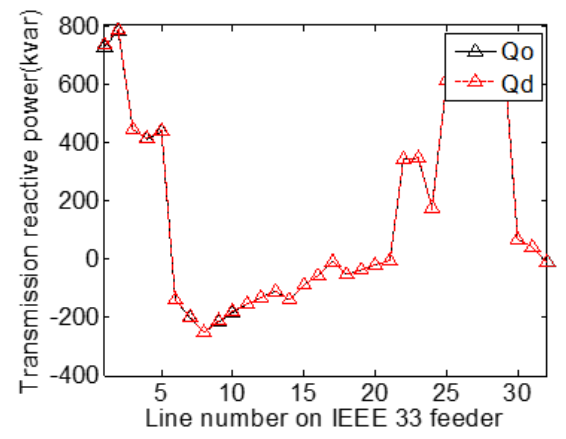

f) Reactive power flow at 13:00

Figure A-48. Comparison between the proposed model and OpenDSS.

\section{Comparison with constant PF control:}

Then, the team compared the results of the proposed optimal PV control and the traditional constant PF control. The two cases were designed as follows:

- Case 1: Constant PF control. The PF is set to 0.95 .

- Case 2: Modified ADMM-based distributed optimal PV control. $\alpha=1.8$ in the over-relaxed ADMM, and adaptive penalty method is used; the maximum number of iterations is set to 900 .

The comparison results are shown in Figure A-49. The simulation results show that in Case 2, the network loss and voltage deviations are smaller, indicating that the proposed model and algorithm are beneficial to secure and economic system operations. 


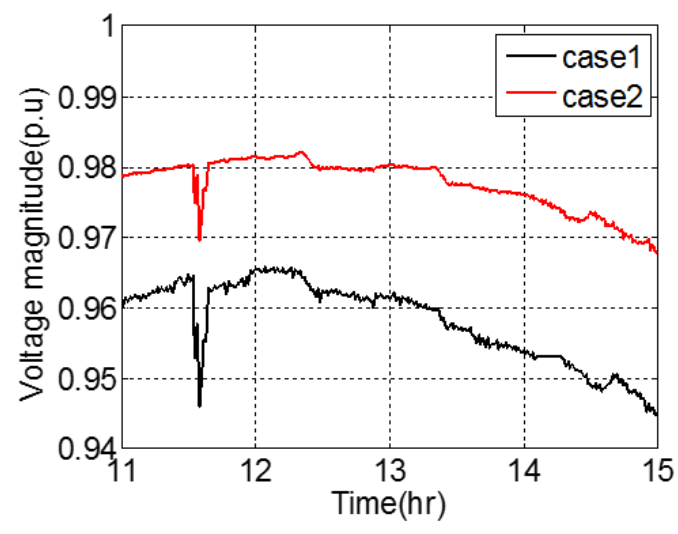

a) Voltage at node 17

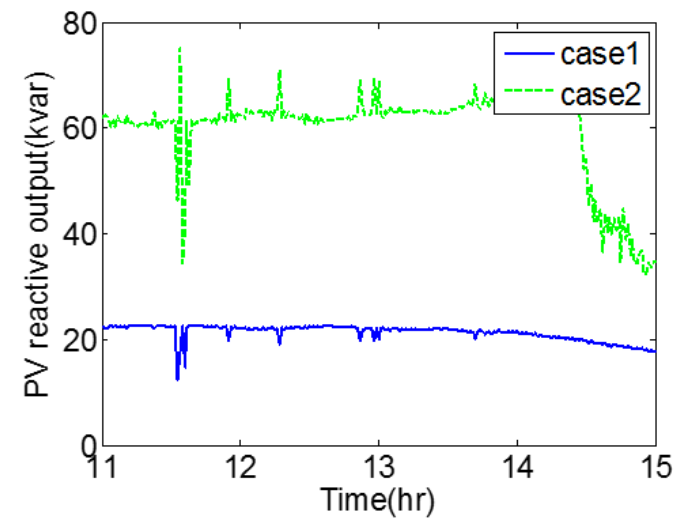

c) Reactive power output at node 17

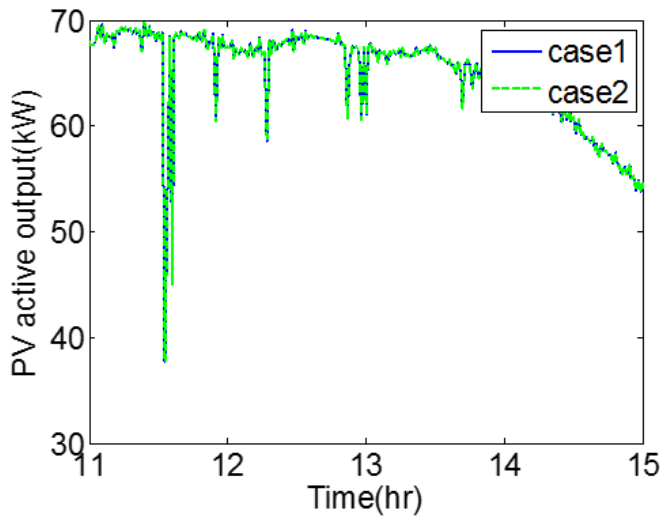

b) Active power output of PV at node 17

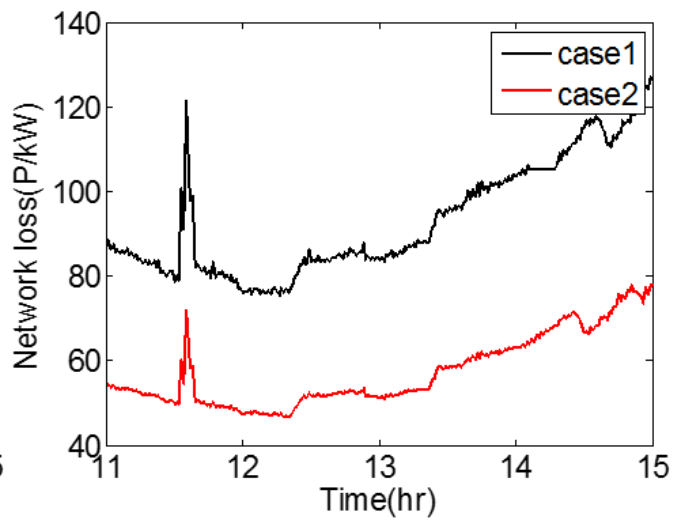

d) Total network loss

Figure A-49. Comparison between the proposed method and constant PF control.

Comparison between with and without building control:

To evaluate the benefits of PV-building coordination, we compare the results in the following two cases.

- Case 1: Distributed optimal PV control without building control.

- Case 2: Distributed optimal PV control in coordination with building control.

The simulation results shown in Figure A-50 indicate that the coordination between PV control and building control can help improve the voltage profiles and reduce total network loss compared with optimal PV control only. 


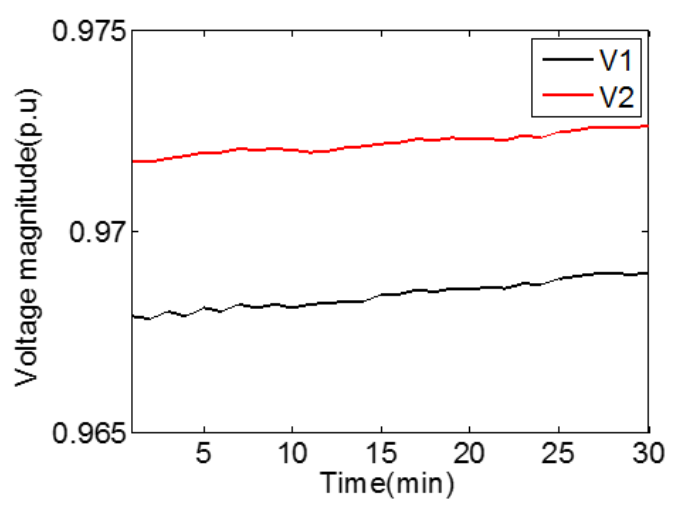

a) Voltage at node 28

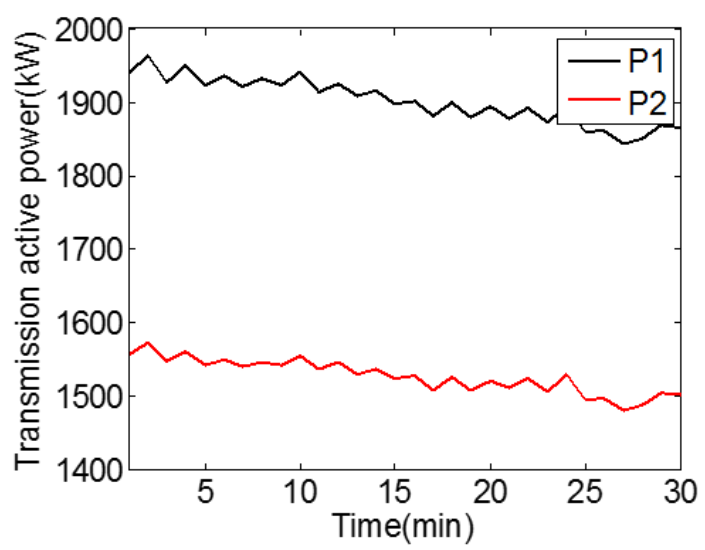

c) Active power flow in line 1-2

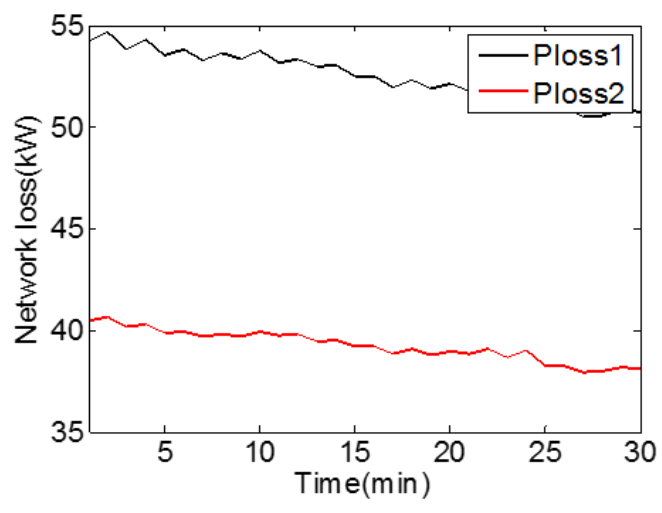

b) Total network loss

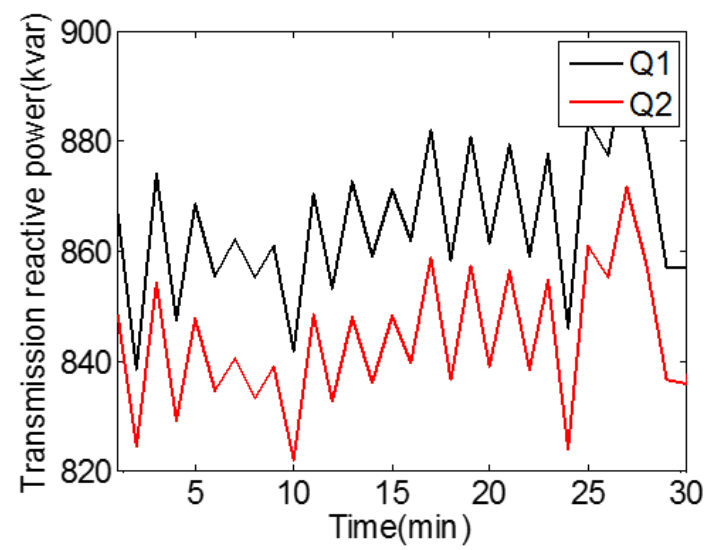

d) Reactive power flow in line 1-2

Figure A-50. Comparison of distributed optimal PV control with and without coordination with building control.

In summary, this work developed a modified ADMM-based algorithm to accelerate the convergence of distributed optimization for PV control. Through simulation and case studies, the proposed model and algorithm were demonstrated to be effective and efficient in the applications of voltage regulations and loss reduction in distribution systems. Additionally, this work designed a mechanism to coordinate the building control with the distributed PV control at different timescales. The simulation results demonstrate that the coordination between PV and building controls can further improve the voltage profile and reduce network losses.

\section{A.5 BUILDING MODEL PARAMETERS ESTIMATION}

System identification is crucial in a specific class of adaptive controllers in which the real-time identification of the underlying system is required [132]. One common approach to system identification in real-time is the class of prediction-error methods, but these are not easily extendable to multiple-input and multiple-output (MIMO) systems. Subspace identification (SID) methods mainly emerged as an alternative [133] approach for estimating MIMO systems. Thus, the team used an SID method.

The dataset used for identification refers to the real-life data collected starting on Tuesday, August 1, 2017 at 10:48:57 a.m. for 16,200 measurement values in Fountain City, Tennessee. The data were collected once every $15 \mathrm{~s}$. The first input is the HVAC state; it takes the discrete values $\{0,1,2\}$, where each value corresponds to the cooling power of the compressor. Accordingly, 0 reflects no cooling 
required $(0 \mathrm{~W}), 1$ reflects stage-one cooling $(4,200 \mathrm{~W})$, and 2 reflects stage-two cooling $(6,800 \mathrm{~W})$. The second input considered is the external outdoor temperature, and the unit used is degrees Fahrenheit. Based on the external temperature and indoor temperature, the HVAC operates at different states with an objective to maintain the indoor temperature within a specified band. The output is the resulting measured indoor temperature. The two inputs and the output for a typical summer day are shown in Figure A-51.
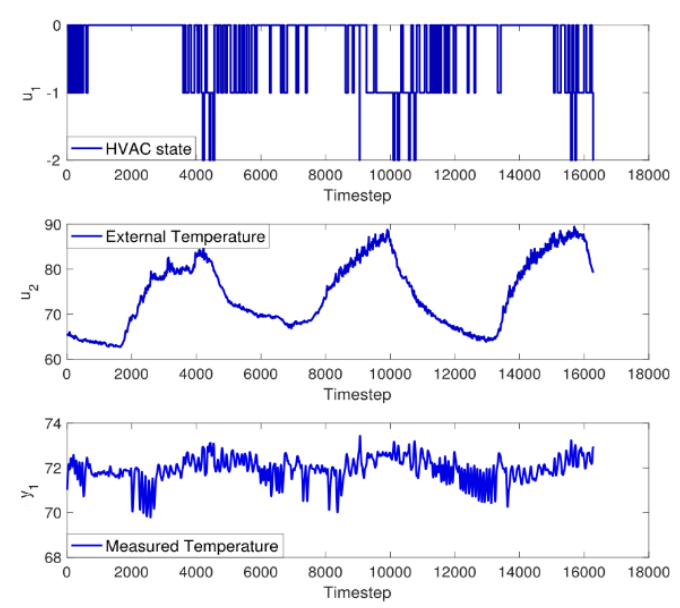

Figure A-51. Inputs and output of the HVAC model system.

If only the first input is considered, then the dataset of just one input and one output is not sufficiently informative to perform system identification. To understand this more clearly, consider the batch of data around the 2,000th time step. For a constant HVAC state, the output seems to be randomly changing. Having external temperature as a second input provides more insight into the system behavior. Hence, the system is modeled as a two-input, one-output system (i.e., MIMO system), and an SID algorithm is employed.

A batch of measurement values from the collected dataset is preprocessed and used to obtain an estimate of the underlying system. The algorithm used to obtain the estimated model is the nuclear norm-based SID (N2SID) that was formulated in Verhaegen and Hansson [134]. Traditional SID methods can be divided into three distinct steps [135]: (1) estimate high-order models, (2) reduce estimated models to lower dimensional subspace, and (3) realize a state-space system from the lower dimensional subspace. Unlike traditional SID methods, N2SID combines the first two steps by embedding the rank minimization criterion (step 2) directly into the identification problem. Essentially, system order estimation is automated. This step of rank minimization is an NP-hard problem, and hence nuclear norm is used as a heuristic for rank minimization [136]. Employing nuclear norm for rank minimization is attractive primarily because it forms a convex envelope on the rank function, rendering the identification problem a convex optimization problem.

To quantify the trade-off between model order reduction and accuracy, a regularization parameter $\lambda$ is employed in N2SID. The other parameter employed in N2SID that can be varied is the length of the dataset, $N_{\text {ide }}$. For this dataset, the team used a batch of 200 measurement values $\left(N_{\text {ide }}=200\right)$ and estimated a model for 15 different values of $\lambda \log$-spaced between 10 and 1,000. The resulting order of the estimated models and the corresponding accuracies are shown in Figure A-52. 

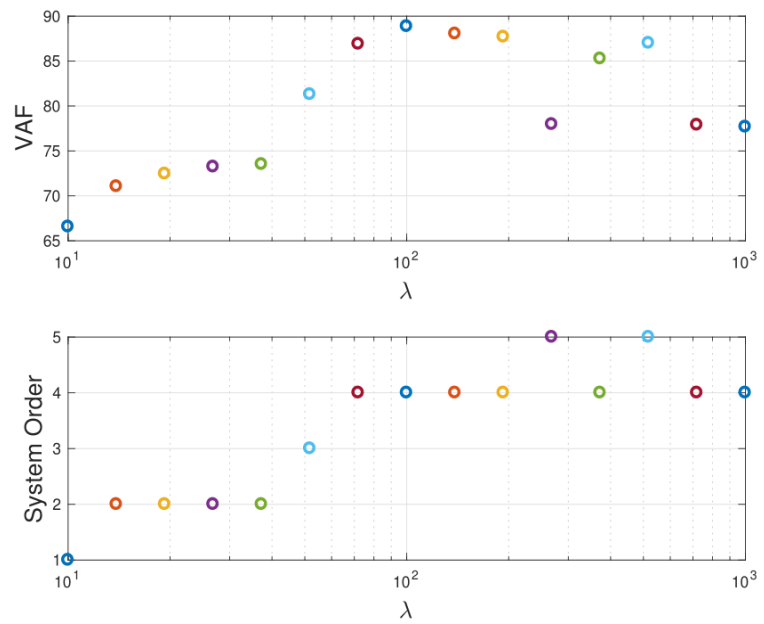

Figure A-52. Evaluating multiple estimated models, $\lambda$ is the regularization parameter of N2SID.

For $\lambda=100$, N2SID estimated a fourth-order model that had the highest accuracy (close to 90\%). The output of this estimated model is compared with the measured output (indoor temperature) in Figure A-53. For the simulation of the estimated model, zero initial state is used, and thus the output starts at a different value from the measured output. Within a few time steps, the behavior of the measured output is reflected in the output of the estimated model.

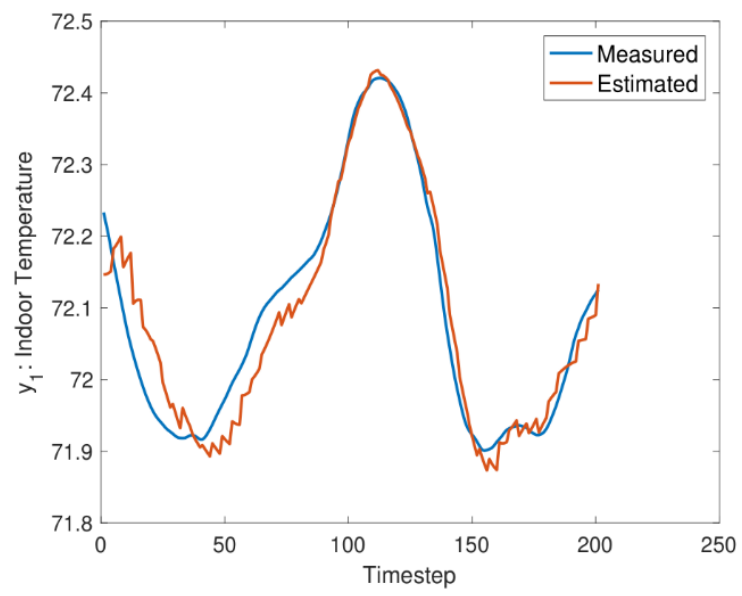

Figure A-53. Accuracy analysis of the estimated model.

The estimated model was accurate up to almost $90 \%$ for the selected batch of the dataset, which was a batch of 200 values. However, if the same model is validated with a larger dataset, then the accuracy of the model deteriorates (Figure A-54). This suggests that a constant simple fourth-order model is not sufficiently comprehensive to capture all the changing dynamics and uncertainties to which the HVAC system might be subjected for a longer period. 

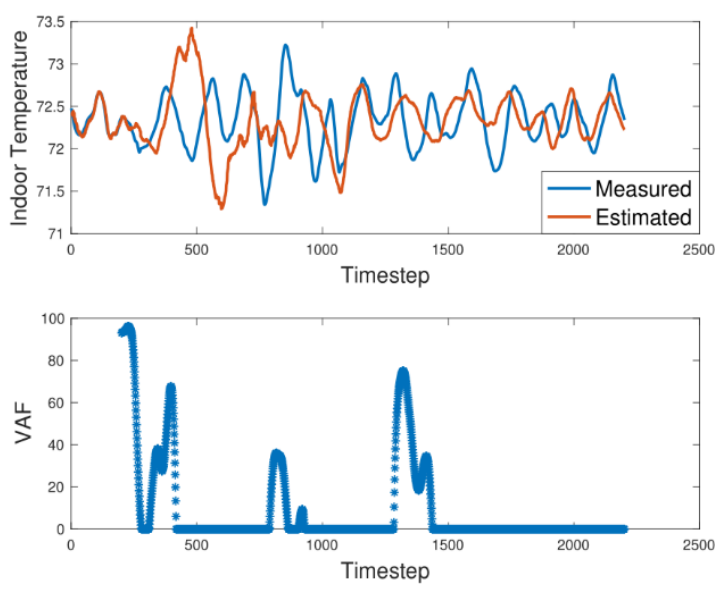

Figure A-54. Validating the entire dataset using a single model.

One way to tackle this problem is to obtain a high-order model that considers the HVAC system dynamics and includes all possible uncertainties. The resulting complicated model still might not be sufficiently exhaustive to cover all feasible scenarios. The other solution is to locally estimate the system every few time steps. The frequency of recursive estimation could be monitored through a criterion, such as if the accuracy of the previously estimated model drops below a certain threshold, and then system identification could be performed. Moreover, the frequency can be adjusted based on the computational constraints that will be imposed in the real-life implementation.

As a demonstration, the system is recursively identified at every time step by using the RN2SID algorithm [137], which is a recursive version of N2SID. The primary feature of RN2SID is that identification at any time step is performed by considering the past system dynamics. The corresponding results are shown in Figure A-55. The accuracy usually remains above or around $80 \%$ and seldom drops below $60 \%$. The order of the estimated model is shown in Figure A-56. Although the estimated models are usually around order 3 , the order is never above 4 . This implies that a recursively estimated model can efficiently capture the local dynamics in a fourth-order model.
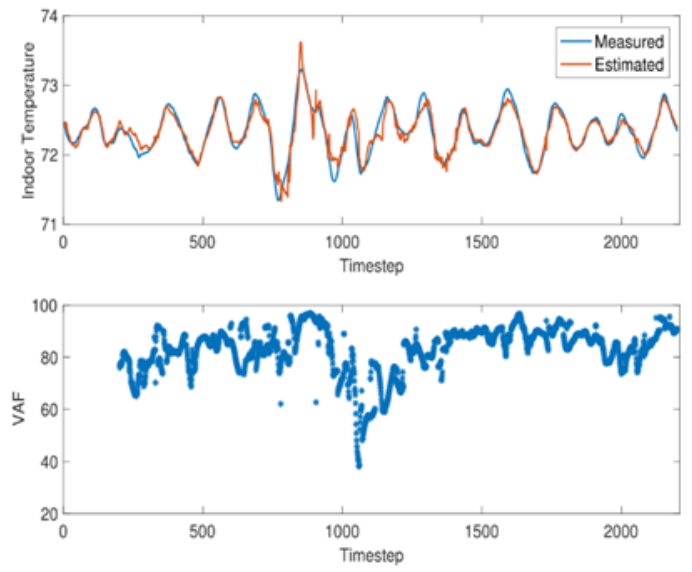

Figure A-55. Accuracy analysis of the model estimated with RN2SID.

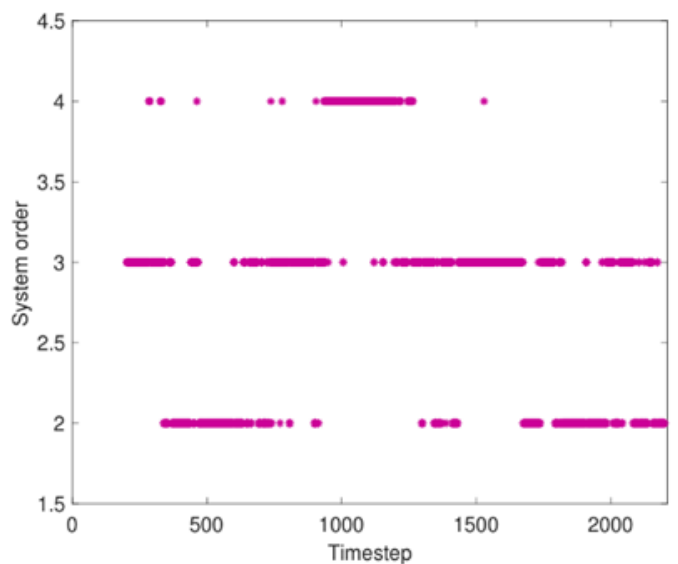

Figure A-56. Order of the model estimated with RN2SID. 
The simulation results presented in Figures A-52 through A-56 are for one-zone HVAC system. The same N2SID method is also tested for a multizone (i.e., four-zone) HVAC system. The dataset employed in this test corresponds to the measurements between August 10, 00:02, and September 3, 00:02, obtained every sampling interval 5 min from the same $\mathrm{CBC}$ church in Knoxville, Tennessee. The estimated model that resulted from system identification is a fifth-order state-space model that is validated through self- and cross-validation. The outputs obtained during self-validation are shown in Figure A-57(a), and the corresponding inputs employed are shown in Figure A-57(b). The self-validation VAF (accuracy) obtained is $80.75 \%$. Cross-validation is performed on the next $20.83 \mathrm{~h}$ of data, and the results are shown in Figures A-58(a)-(b). The cross-validation VAF (accuracy) obtained is 50.57\%. The results are summarized in Table A-4.

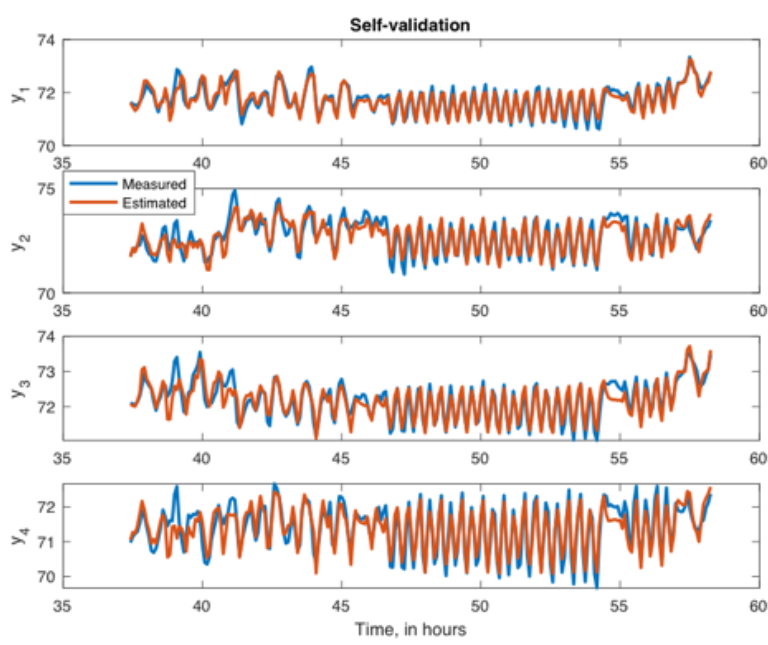

(a)

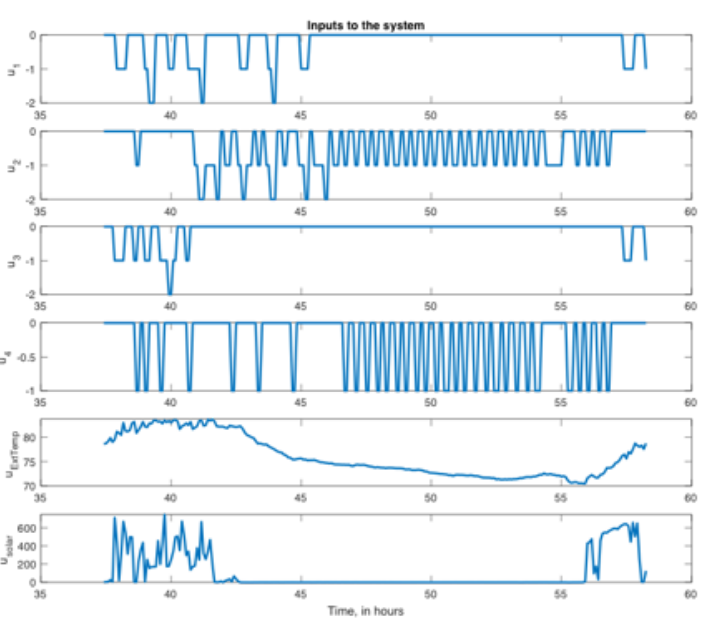

(b)

Figure A-57. (a) Self-validation and (b) inputs given to the plant and the estimated model.

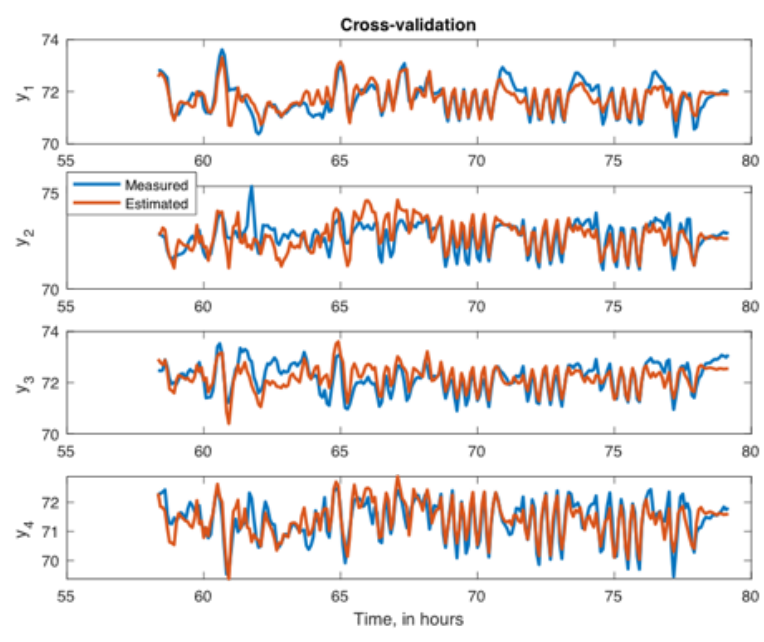

(a)

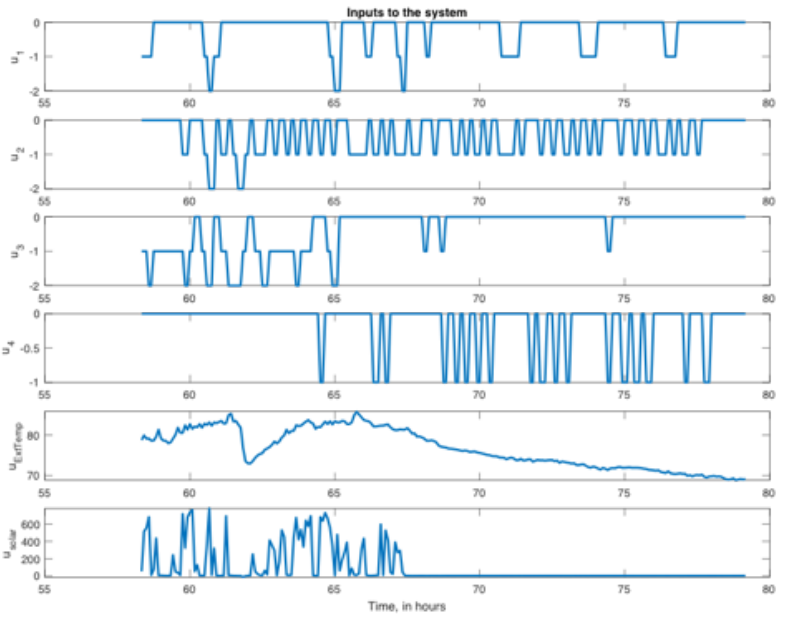

(b)

Figure A-58. (a) Cross-validation and (b) inputs given to the plant and the estimated model. 
Table A-4. Evaluation of the accuracy of the estimated model.

\begin{tabular}{|c|c|c|}
\hline Error & $\begin{array}{c}\text { Self-validation } \\
\left({ }^{\circ} \mathbf{F}\right)\end{array}$ & $\begin{array}{c}\text { Cross-validation } \\
\left({ }^{\circ} \mathbf{F}\right)\end{array}$ \\
\hline RMSE & 0.2779 & 0.4470 \\
\hline MAE & 0.2157 & 0.3466 \\
\hline $\begin{array}{c}\text { Maximum } \\
\text { error }\end{array}$ & 1.4852 & 3.2718 \\
\hline
\end{tabular}

Results showed that highly accurate thermal building models can be estimated by using the SID mechanism. However, this mechanism requires many computations that might slow its implementation and deployment processes. Thus, the team proposes a much simpler estimation mechanism, which is based on least squares (LS) methods [138] in which the indoor room temperatures are estimated by solving for $x$ in $Y=A x$, where $Y$ and $A$ contain previous measurement values, and $x$ is the vector of unknown coefficients. The estimated building model parameters are used to predict indoor temperatures that are needed for the developed building load controller to provide improved performance. Figure A-59 illustrates the predicted indoor temperatures for different prediction horizons using LS mechanism. High prediction accuracy was achieved for short-term prediction horizons (less than $10 \mathrm{~min}$ ), and the accuracy deteriorates for longer prediction horizons. Figure A-60 illustrates the root mean square error (RMSE) of indoor temperature prediction for different prediction horizons using LS and compares it with the SID method. The SID method provides higher accuracy but requires larger computations.

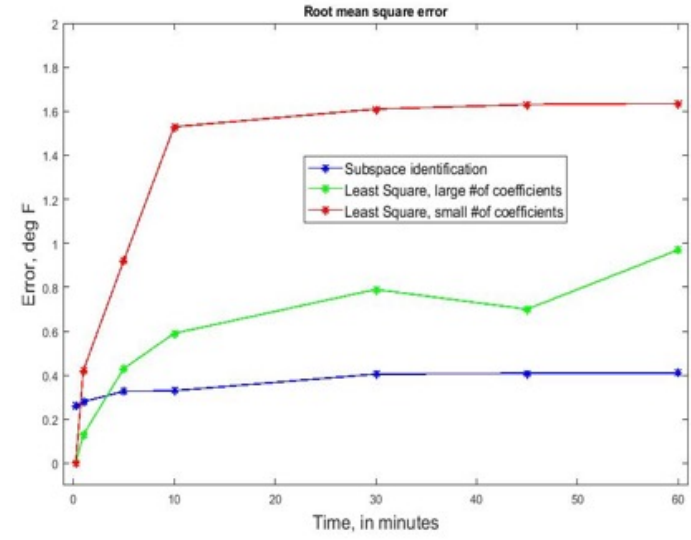

Figure A-59. RMSE of indoor temperature prediction for different prediction horizons using LS and compared with SID.
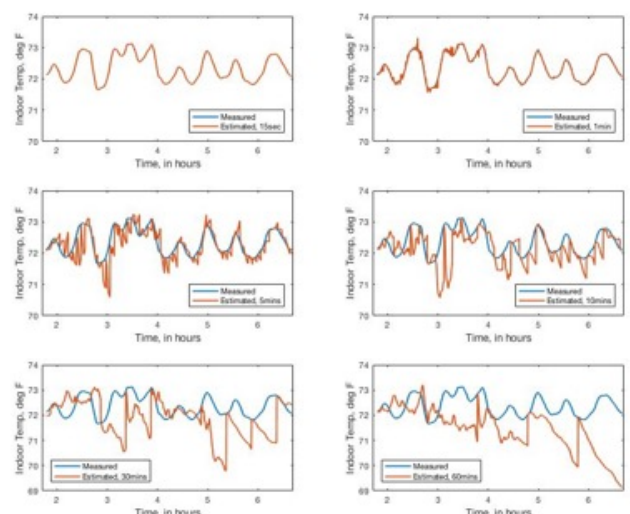

Figure A-60. Prediction of indoor temperatures using LS for several prediction horizons: $15 \mathrm{~s}, 1 \mathrm{~min}$, 5 mins, 10 mins, 30 mins, and 60 mins.

\section{A.6 PV POWER FORECAST}

In summary, the team reviewed the HVAC system dataset and decided to employ an SID algorithm accordingly. After obtaining satisfactory results for a batch of data, the team employed the estimated model to describe the HVAC dynamics. Because of uncertainties associated with the HVAC system, the team concluded that resorting to recursive identification was more appropriate, and the results were observed to be satisfactory. 
The real-time prediction of PV power output is necessary for overcoming the temporal variability challenges of solar radiation. Previous attempts to model solar irradiance can be classified into three general types [139]: physical, frequency distribution, and stochastic. The physical approach studies the physical processes that occur in the atmosphere and that influence solar radiation. It is exclusively based on physical considerations, allowing that the radiant energy exchanges occur within the earth-atmosphere system. This approach dictates models that account for the estimated solar irradiation at the ground in terms of a certain number of physical parameters, such as water vapor content, dust, aerosols, and clouds and cloud types [140]. For each place and period of the year, the frequency distribution approach provides a descriptive statistical analysis of the main quantities of interest, such as hourly or daily global, diffuse or beam solar irradiation, and statistical modeling of the observed empirical frequency distributions. The stochastic modeling approach is more flexible and can incorporate any nondeterministic influences, such as cloud movement and pollution levels, into the model, as well as any nonstandard features, such as shading specific to a particular location [139]. Thus, stochastic modeling can capture the intrinsically nondeterministic (i.e., uncertain) nature of irradiance fluctuations. For example, understanding the subminute behavior of PV generation will be necessary for developing realistic predictive models (i.e., controllers) for building HVAC systems.

Since the last decade, stochastic time series forecasting has been applied to different applications with higher degrees of success. A time series is a collection of time-ordered observations $x_{t}$, each being recorded at a specific time $t$ (period) [141]. Time series can appear in a wide set of domains, such as finance, production, or control. As a first approximation, a time series model assumes that past patterns will occur in the future. A time series model could be used to provide synthetic time series statistically similar to the original one. Time series modeling begins with the selection of a suitable mathematical model or class of models for the data. Then, future values of measurements [142] can be predicted. Moreover, time series methods have been applied to forecast solar PV power in several other works [139, 143-145]. These works provide accurate models and predictions that capture the hourly, daily, and seasonal solar PV trends.

An AR model is a type of random process that is often used to model and predict various types of natural phenomena. A typical AR process can be written as:

$$
X(k)=\sum_{i=1}^{p} \alpha_{i} X(k-i)+\epsilon(k)
$$

where $X(k)$ is a time series, $\alpha_{1}, \ldots, \alpha_{p}$ are parameters of the model corresponding to $X(k)$, and $\epsilon(k)$ represents the uncertain term or fluctuations modeled as white noise at time $k . p$ is the order of the AR model that represents how many past measurements upon which the current one depends. If $X(k)$ is used to represent the solar PV power metrics, then the future metrics can be predicted by Eq. (A-38). Before prediction, the parameters in Eq. (A-38) must be estimated based on the past metrics by using the LS mechanism. After model parameters are estimated, one-step prediction of the metrics can be computed by:

$$
\hat{X}(k+1)=\sum_{i=1}^{p} \alpha_{i} X(k+1-i)
$$

The AR integrated moving average (MA) (ARIMA) time series model is an extension of the AR model that comprises the AR model, integrating part 1, and the MA model. ARIMA is considered a good prediction model for forecasting the future values of provided time series with stable input variables. The ARIMA model refers to $(p ; i ; q)$, where $p$ represents the order of the AR model, $i$ represents the order of the differencing part, and $q$ represents the order of the MA model. A typical ARIMA $(1,1,1)$ model can be expressed as:

$$
z_{t}=\alpha+\emptyset z_{t-1}+\theta \epsilon_{t-1}+\epsilon_{t}
$$


where $z_{t}$ is the difference between the current value $X(k)$ and the previous value $X(k-1)$. The $(1,1,1)$ in the equation are the autoregressive $\left(z_{t}\right)$, differencing part, and MA $\left(\epsilon_{t}\right)$ lag orders, respectively. The intuitive understanding of Eq. (A-40) is straightforward. The current value of the time series $z_{t}$ will depend on the past value of the series $z_{t-1}$ and will correct itself to the error made in the last time period $\epsilon_{t-1}$. The initial differencing step that corresponds to the "integrated" part of the model is applied to eliminate any nonstationarity in the original data $X(k)$. The standard statistical methodology to construct AR and ARIMA models includes the steps listed in Table A-5. A detailed explanation for each step can be found in Ji and Chee [142]. This stochastic framework is selected to predict PV production and avoid requiring exogenous influential features, namely the cloud cover and temperature.

Table A-5. The standard statistical methodology to construct AR and ARIMA models.

\begin{tabular}{|c|l|}
\hline Step number & Task \\
\hline Step 0 & A class of models is formulated, assuming certain hypotheses. \\
\hline Step 1 & A model is identified for the series considered. \\
\hline Step 2 & The parameters of the model are estimated. \\
\hline Step 3 & $\begin{array}{l}\text { If the hypotheses of the model are validated, then the procedure continues in } \\
\text { Step 4; otherwise, the procedure continues in Step 1 to refine the model. }\end{array}$ \\
\hline Step 4 & The model is used to forecast. \\
\hline
\end{tabular}

The team tested the prediction algorithms on 12 month measurement of PV power output data collected from $13 \mathrm{~kW}$ PV panel at Distributed Energy Communications and Controls lab. The PV power data are downsampled to $10 \mathrm{~min}$ intervals to accommodate the controller time steps, which are every $10 \mathrm{~min}$.

Figures A-61 and A-62 demonstrate the performance of the $\operatorname{AR}(2)$ and $\operatorname{ARIMA}(2,1,2)$ prediction algorithms acting on real solar PV power output data for a particular summer. The PV power was predicted with high accuracy. The RMSE for the AR(2) model is 775, and the RMSE for the $\operatorname{ARIMA}(2,1,2)$ model is 759 . Because both models provide comparable performance, the team plans to use the simpler one, $\operatorname{AR}(2)$, in the implementation phase.

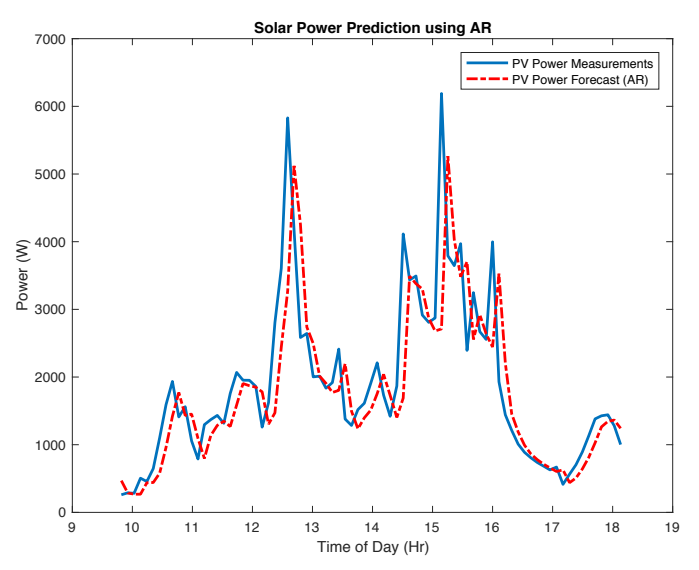

Figure A-61. Performance of PV power output prediction using $A R(2)$.

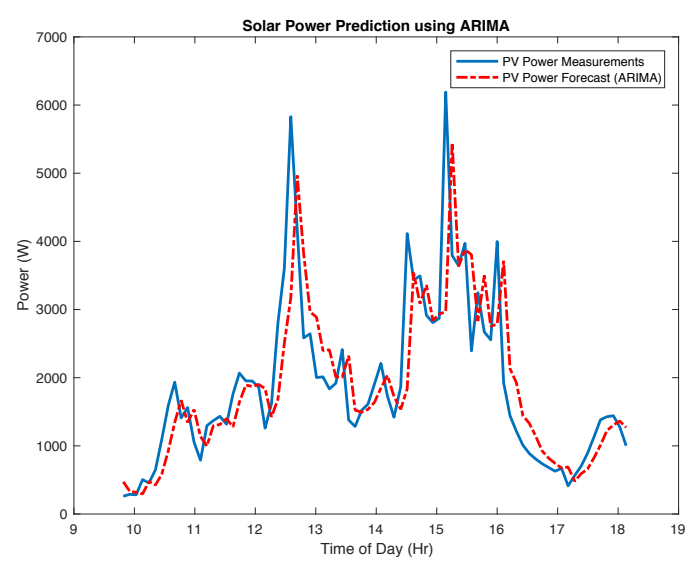

Figure A-62. Performance of PV power output prediction using ARIMA(2,1,2). 


\section{A.7 ADAPTIVE BUILDING LOAD CONTROL (MFC)}

\section{A.7.1 MFC Development}

This section discusses how the team used the MFC algorithm developed in Fliess and Join [146] as an alternative control strategy to MPC to allow most of the generated PV power to be consumed locally by building loads while maintaining occupant comfort. MPC methodologies are designed to handle constraints efficiently, but they are associated with relatively high computational cost. In contrast, MFC uses an ultra-local model (i.e., approximation) of the system that is estimated in real time. It circumvents the daunting task of deriving reliable building models and accounts for all the uncertainties and unknown weather disturbances and changes in occupancy pattern [147-150]. Thus, it is computationally efficient and easily deployable on small, embedded devices, and it can be implemented in real time or near-real time.

Like MPC strategies, the objective is to employ all the generated solar PV energy to control the indoor temperatures of the buildings. Here, the generated PV energy is accessible by all the considered buildings because the energy is divided among the community. A comfort band was defined for the desirable indoor temperatures, and the team's goal was to consume most of the generated energy without jeopardizing occupant comfort. This would minimize the impact on the grid, reduce the size of storage devices, and increase solar PV penetration levels.

As described in Eqs. (17)-(18), the thermal HVAC system is modeled as a single-input and single-output linear state-space representation. This HVAC model can be approximated by an ultra-local model as:

$$
\dot{y}=F+\alpha u,
$$

where $u$ and $y$ are the input and output of the system, respectively, and $F$ describes the poorly known or unknown parts of the system. The parameter $\alpha$ corrects the difference in the magnitudes of the input and output. $F$ is approximated by a piecewise constant function $\varphi$ that is given as:

$$
\varphi=\frac{-6}{L^{3}} \int_{t-L}^{t}[(L-2 \sigma) y(\sigma)+\alpha \sigma(L-\sigma) U(\sigma)] d \sigma .
$$

$\varphi$ is estimated by using the measurements of the system obtained in the last $L$ seconds, and $F$ is continuously updated accordingly. Using the latest $F$, the intelligent-proportional control law is given by:

$$
u=-\frac{F-\dot{y}^{*}+K_{p}\left(y-y^{*}\right)}{\alpha} \text {. }
$$

Here, $y^{*}$ is the desired reference trajectory, and $K_{p}$ is the proportional gain. Combining 7 and 9 provides the error dynamics:

$$
\dot{e}+K_{p} e=0
$$

where $e=y-y^{*}$ is the tracking error. The value of $K_{p}$ is obtained from the solution of the differential equation in Eq. (A-44) and is updated at every time step. Therefore, the only parameters that must be manually set are $\alpha$ and $L$, making the control design straightforward. The MFC method uses an ultra-local model that is estimated from the input and output measurements. Therefore, the state-space HVAC model is only used to simulate the building conditions and is not used in the design of the controller.

The objective is to control the indoor temperatures of $N$ number of buildings by using the generated solar PV energy. This implies that the generated energy is divided among all $N$ buildings. Let the generated 
solar PV energy be denoted as $E$. The constraint of employing all of the generated energy can be mathematically expressed as:

$$
E(t)-\varepsilon \leq \sum_{k=1}^{N} E_{k}(t) \leq E(t)+\varepsilon,
$$

where $E_{k}(t)$ is the energy consumed by the $k$ th building at time $t$, and $\varepsilon$ is the permissible tolerance in the tracking error. From the dynamics of the building HVAC model, $E_{k}$ is given as $E_{k}=3.5 u_{k}$, where $u_{k}$ is the control input of the $k$ th building HVAC system. Moreover, because the HVAC systems can only be turned on or off, there is a constraint on the control input: $u=\{0,1\}$, where 0 and 1 correspond to the HVAC system turned off and on, respectively. Because the team's focus included fully employing the generated PV energy, the analysis was limited to during the day when there is sufficient solar energy. Accordingly, when the HVAC system is turned off, the indoor temperature of the building increases, and when the HVAC system is turned on the building temperature decreases. The HVAC system considered here operates only in cooling mode.

The methodology to achieve the aforementioned objective is sequentially summarized in the following steps:

1. For each building $k$ at each time step $t$, feed the previous $L$ seconds of measurements to the MFC; that is, $\left.u_{k}(t+1)=\operatorname{MFC}\left(u_{k}(t-L): t\right) ; y_{k}((t-L): t)\right)$.

2. Calculate the corresponding energy that will be consumed with $u_{k}(t+1)$ as the input; that is, $E_{k}(t+1)=3.5 u_{k}(t+1)$.

3. Check whether the total energy to be consumed satisfies or violates the constraint Eq. (A-45).

4. If the constraint in Eq. (A-45) is satisfied, then the control input to the $k$ th building is $u_{k}(t+1)$; round off to satisfy $\{0,1\}$.

5. If the constraint in Eq. (A-45) is violated, then decide the buildings that most require cooling based on the value of $u_{k}(t+1)$. For example, if $u_{29}=0.9$ and $u_{87}=0.7$, then the 29th building will be turned on at this time step.

6. The previous step is performed until all the available energy is allotted.

As a further note to step 5, consider the case in which there are multiple buildings $\left(N_{1}\right.$ number of buildings) that have the same value of $u_{k}(t+1)$. Because the generated energy is not enough to cool all $N_{1}$ buildings, $N_{2}<N_{1}$ number of buildings are cooled, and the remainder are buffered for the next time step. Here, $N_{2}$ corresponds to the number of buildings that can be cooled by using the generated energy. When the available energy is more than the required energy, the aforementioned conflict resolution technique is mirrored and employed.

\section{A.7.2 Performance Analysis}

In this section, $\alpha=1, L=3,600 \mathrm{~s}$, the sampling time is $600 \mathrm{~s}$, and $\varepsilon$ is set as $10 \%$ of $E$. The external temperature and solar irradiance measurements are selected for a typical summer day in July 2017 in Knoxville, Tennessee. $N=100$ nonidentical buildings, starting at different initial conditions. To generate $N$ nonidentical buildings, the team sampled from a normal distribution in which the state-space model parameters are considered as the mean. $N$ values for each state-space parameter are sampled from the normal distributions, and each set is used as a model for the building. The MFC method is used in combination with the reviewed methodology to control the indoor temperatures while employing all the 
generated energy. Because the proportional gain $K_{p}$ is automatically computed based on the tracking error of the indoor temperature, it does not need to be manually tuned, even when the models are different. The desired indoor temperature is $23^{\circ} \mathrm{C}$, and the comfort band is defined as $23 \pm 1.5^{\circ} \mathrm{C}$. The indoor temperatures obtained in the 100 buildings with MFC are shown in Figure A-63(a), and the temperatures obtained using MPC under the same conditions as in MFC are shown in Figure A-63(b). The corresponding total energy consumed at each time step for both methods is compared in Figure A-63(c).

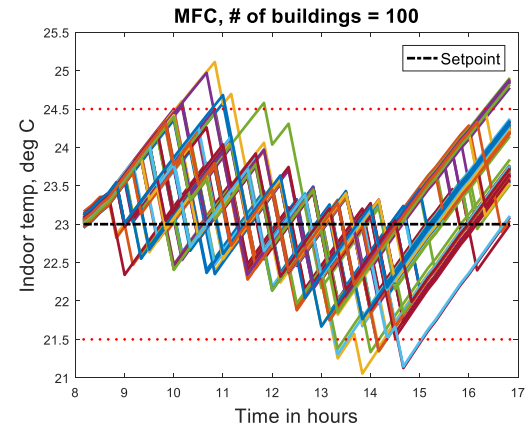

(a)

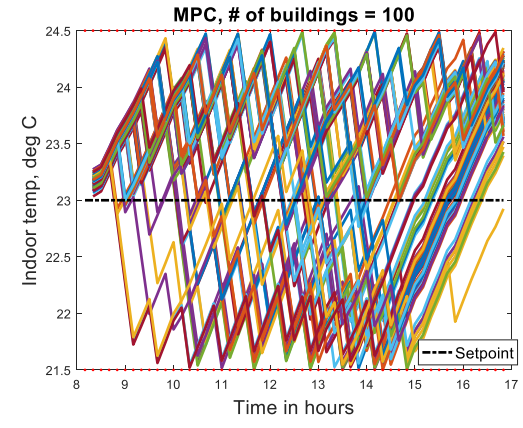

(b)

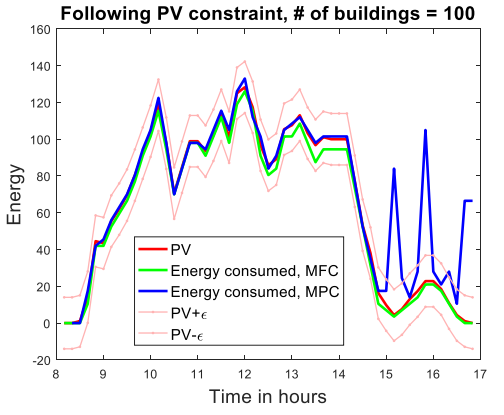

(c)

Figure A-63. Indoor temperatures obtained by using (a) MFC, (b) MPC, and (c) total energy consumption by 100 buildings obtained with MFC and MPC vs. generated PV energy.

Although MPC strictly maintains the indoor temperatures within the comfort band, the energy consumed is higher than the generated energy for a brief time. On the other hand, although MFC uses the generated energy within permissible limits, the indoor temperatures for a few buildings deviate from the comfort band by less than $0.5^{\circ} \mathrm{C}$ for a brief time. This is because the control algorithm implemented with MPC imposes hard constraints on maintaining the indoor temperature, whereas MFC imposes hard constraint on employing the generated energy within permissible limits. If following the energy constraint is more important than maintaining the indoor temperature, then the MPC algorithm can be easily reconfigured in a straightforward manner, whereas MFC is not as flexible with changing the constraints. The results are summarized in Table A-6 using different tracking error metrics.

Table A-6. Comparison of MFC and MPC using tracking error metrics.

\begin{tabular}{|c|c|c|c|c|}
\hline \multirow{2}{*}{ Tracking error metric } & \multicolumn{2}{|c|}{ Indoor temperature $\left({ }^{\circ} \mathbf{C}\right)$} & \multicolumn{2}{c|}{ Energy consumption (kW) } \\
\cline { 2 - 5 } & MFC & MPC & MFC & MPC \\
\hline RMSE & 0.60 & 0.82 & 3.64 & 20.94 \\
\hline MAE & 0.49 & 0.74 & 2.97 & 8.22 \\
\hline Maximum error & 2.11 & 1.50 & 9.24 & 82.17 \\
\hline
\end{tabular}

The duty cycle and number of HVAC state switching (from the on state to the off state or from the off state to the on state) for this simulation scenario for MFC and MPC are illustrated in Figures A-64 and A-65, respectively. These results are summarized in Table A-7. For this simulation scenario, MFC provides a duty cycle comparable with MPC with a smaller number of HVAC state switching. 
Duty Cycle with MFC

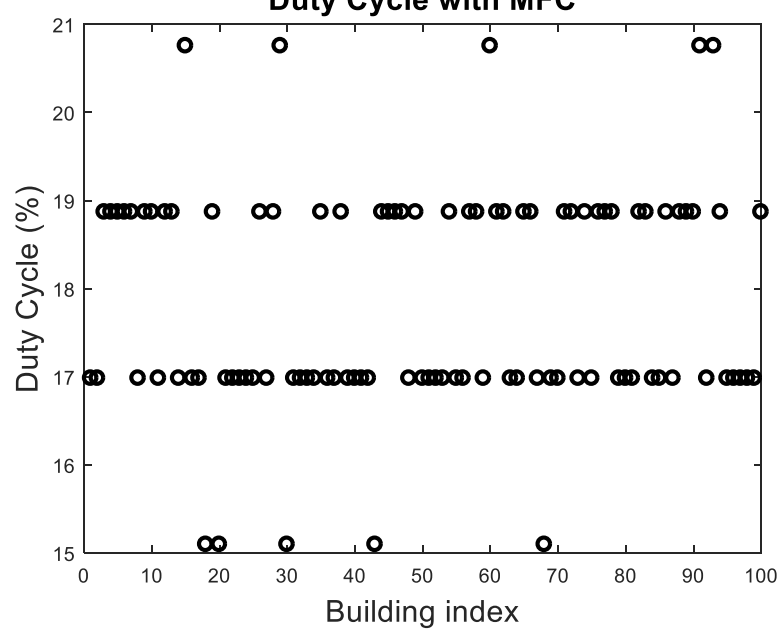

(a)

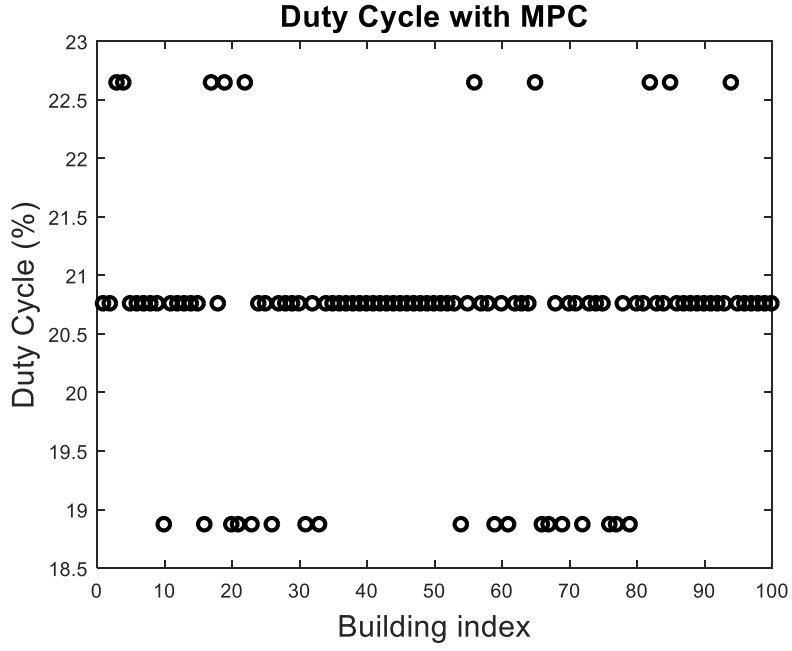

(b)

Figure A-64. The duty cycles for 100 HVAC systems using (a) MFC and (b) MPC.

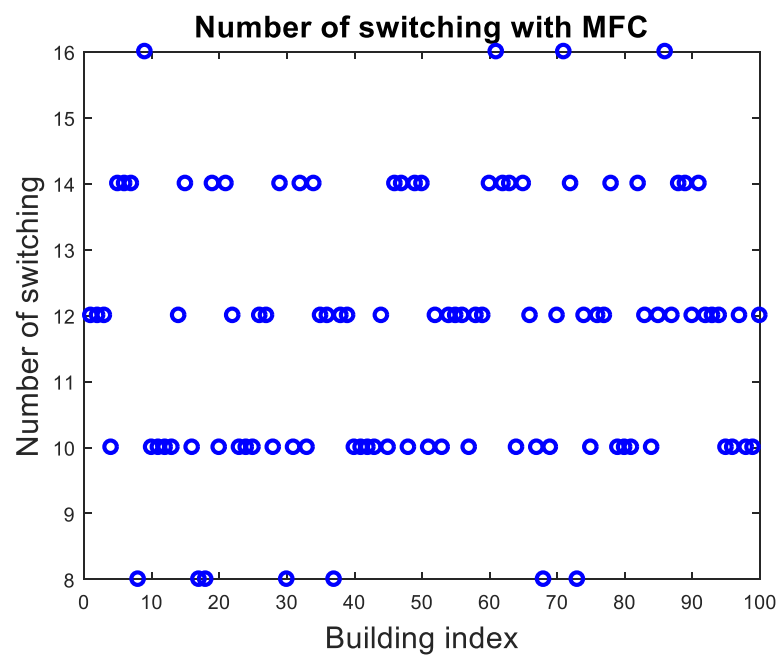

(a)

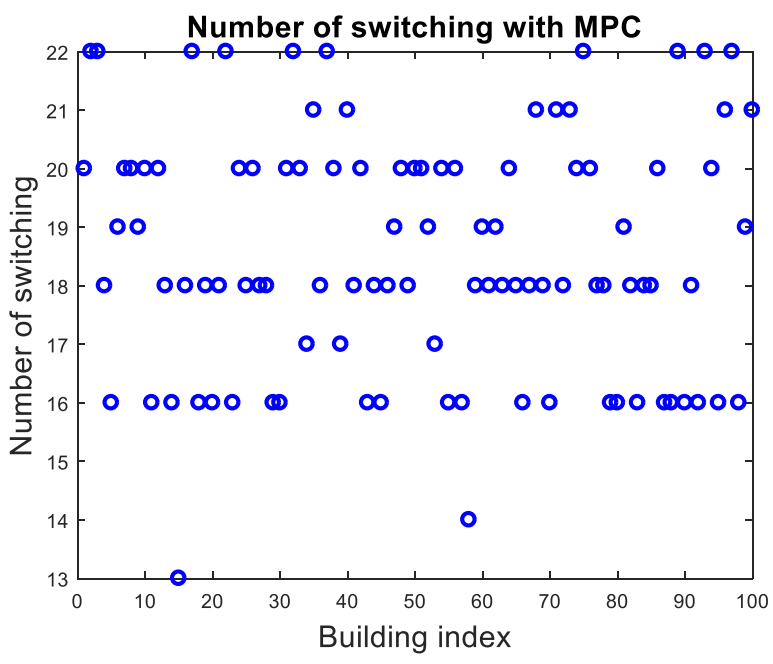

(b)

Figure A-65. The total number of state switching for 100 HVAC systems using (a) MFC and (b) MPC.

Table A-7. Comparison of MFC and MPC in terms of HVAC duty cycle and number of state switching.

\begin{tabular}{|c|c|c|c|c|}
\hline \multirow{2}{*}{ Amount } & \multicolumn{2}{|c|}{ Duty cycle (\%) } & \multicolumn{2}{c|}{ Number of switching } \\
\cline { 2 - 5 } & MFC & MPC & MFC & MPC \\
\hline Minimum & 15.09 & 18.87 & 6 & 13 \\
\hline Average & 17.81 & 20.60 & 11 & 18 \\
\hline Maximum & 22.64 & 22.64 & 16 & 22 \\
\hline
\end{tabular}

Because of the comparable performance of MFC compared with MPC but with much less computational requirements, the team used the MFC strategy as a backup plan in the implementation and deployment phase, especially when the computational complexity was not feasible (i.e., there was a scalability issue). 


\section{A.7.3 Stability Analysis}

The MFC system is asymptotically stable for continuous-time input. The asymptotic stability of the system is given under the conditions that the control input from Eq. (A-43) is fed to the system. However, in the case of controlling building indoor temperature with a HVAC unit that can be either only turned on or off, there are limitations on the control input. Therefore, the team needed to investigate the stability conditions for the case in which the control input is a discrete value. The constraints on the control input are embedded as follows. Let $u$ be the value obtained from Eq. (A-43) and $u_{r}$ be the control input actually fed to the system in Eq. (A-41). $u_{r}$ is obtained from $u$ as:

$$
u_{r}= \begin{cases}0 & \text { if } u<0.5 \\ 1 & \text { if } u \geq 0.5\end{cases}
$$

Because $u_{r}$ is supplied to the system, Eq. (A-41) will be transformed as:

$$
\dot{y}=F+\alpha u_{r}
$$

Case 1: $u<0.5$ :

$$
\Rightarrow \frac{-1}{\alpha}\left[F-\dot{y}^{*}+K_{p} e\right]<0.5
$$

Because $u_{r}=0$ in this case, Eq. (A-47) gives $\dot{y}=F$ :

$$
\begin{gathered}
\Rightarrow \frac{-1}{\alpha}\left[\dot{y}-\dot{y}^{*}+K_{p} e\right]<0.5 \\
\Rightarrow \frac{-1}{\alpha}\left[\dot{e}+K_{p} e\right]<0.5 \\
\Rightarrow \dot{e}+K_{p} e>-0.5 \alpha .
\end{gathered}
$$

Case 2: $u \geq 0.5$

$$
\Rightarrow \frac{-1}{\alpha}\left[F-\dot{y}^{*}+K_{p} e\right] \geq 0.5
$$

Because $u_{r}=0$ in this case, Eq. (A-47) gives $\dot{y}=F+\alpha$ :

$$
\begin{gathered}
\Rightarrow \frac{-1}{\alpha}\left[\dot{y}-\alpha-\dot{y}^{*}+K_{p} e\right] \geq 0.5 \\
\Rightarrow-\left[\dot{e}+K_{p} e\right]+\alpha \geq 0.5 \alpha \\
\Rightarrow \dot{e}+K_{p} e \leq 0.5 \alpha .
\end{gathered}
$$

Combining Eqs. (A-48) and (A-49) results in:

$$
-0.5 \alpha<\dot{e}+K_{p} e \leq 0.5 \alpha
$$

Therefore, when the control input is continuous, $\dot{e}+K_{p} e=0$. The solution of this differential equation is:

$$
e(t)=e\left(t_{0}\right) \exp \left(-K_{p}\left(t-t_{0}\right)\right)
$$


where $\exp (\cdot)$ is the exponential function, and $t_{0}$ is the initial time. Without loss of generality, let $t_{0}=0$. From Eq. (A-51), the system is asymptotically stable when the control input is unconstrained. When the control input is constrained, as in Eq. (A-46), the error dynamics are as in Eq. (A-50). To further investigate the stability conditions for constrained control input, the differential inequality in Eq. (A-50) must be solved. The team employed the results of the following lemma [151] to obtain the solution to Eq. (A-50).

Lemma: Let $x_{0}, y_{0} \in \mathbb{R}, I=\left[x_{0}, \infty\right\}, a, b \in C(I), y \in C^{1}(I)$ and:

$$
\dot{y} \leq a(x) y(x)+b(x), \quad \forall x \geq x_{0}, y\left(x_{0}\right)=y_{0} .
$$

Then the following holds:

$$
y(x) \leq y_{0} \exp \left(\int_{x_{0}}^{x} a(t) d t\right)+\int_{x_{0}}^{x} b(s) \exp \left(\int_{s}^{x} a(t) d t\right) d s, \quad \forall x \geq x_{0}
$$

If the converse holds in Eq. (A-52), then the converse holds in Eq. (A-53) also. Consider the right-hand part of Eq. (A-50):

$$
\begin{aligned}
& \dot{e}(t)+K_{p} e(t) \leq 0.5 \alpha \\
\Rightarrow & \dot{e}(t) \leq-K_{p} e(t)+0.5 \alpha .
\end{aligned}
$$

Using the results of the lemma, the team obtained:

$$
\begin{gathered}
e(t) \leq e(0) \exp \left(\int_{0}^{t}-K_{p} d \tau\right)+\int_{0}^{t} 0.5 \alpha \exp \left(\int_{s}^{t}-K_{p} d \tau\right) d s \\
\Rightarrow e(t) \leq e(0) \exp \left(-K_{p} t\right)+\int_{0}^{t} 0.5 \alpha \exp \left(-K_{p}(t-s)\right) d s \\
\Rightarrow e(t) \leq e(0) \exp \left(-K_{p} t\right)+\frac{0.5 \alpha}{K_{p}} \exp \left(-K_{p} t\right)\left[\exp \left(K_{p} t\right)-1\right] \\
\Rightarrow e(t) \leq e(0) \exp \left(-K_{p} t\right)+\frac{0.5 \alpha}{K_{p}}\left[1-\exp \left(-K_{p} t\right)\right]
\end{gathered}
$$

Similarly, consider the left-hand part of Eq. (A-50):

$$
\begin{gathered}
-0.5 \alpha<\dot{e}(t)+K_{p} e(t) \\
\Rightarrow \dot{e}(t)>-K_{p} e(t)-0.5 \alpha .
\end{gathered}
$$

Using the results of the lemma, the team obtained:

$$
\begin{gathered}
e(t)>e(0) \exp \left(\int_{0}^{t}-K_{p} d \tau\right)-\int_{0}^{t} 0.5 \alpha \exp \left(\int_{s}^{t}-K_{p} d \tau\right) d s \\
\Rightarrow e(t)>e(0) \exp \left(-K_{p} t\right)-\frac{0.5 \alpha}{K_{p}}\left[1-\exp \left(-K_{p} t\right)\right] .
\end{gathered}
$$

Combining Eqs. (A-54) and (A-55), the error dynamics for the case when the control input is constrained as in Eq. (A-46) is given by:

$e(0) \exp \left(-K_{p} t\right)-\frac{0.5 \alpha}{K_{p}}\left[1-\exp \left(-K_{p} t\right)\right]<e(t) \leq e(0) \exp \left(-K_{p} t\right)+\frac{0.5 \alpha}{K_{p}}\left[1-\exp \left(-K_{p} t\right)\right]$ 
By comparing Eq. (A-56) with the error dynamics associated with unconstrained control input, that is:

$$
e(t)=e(0) \exp \left(-K_{p} t\right)
$$

it can be observed that Eq. (A-56) is within $\pm \frac{0.5 \alpha}{K_{p}}\left[1-\exp \left(-K_{p} t\right)\right]$ of Eq. (A-50). As $t \rightarrow \infty$, $\left[1-\exp \left(-K_{p} t\right)\right] \rightarrow 1$. Therefore, even when the control input is constrained, the error will be within $\pm \frac{0.5 \alpha}{K_{p}}$ of the error obtained with unconstrained input. As noted previously, MFC leads to an asymptotically stable system when the control input is unconstrained. Because the error in the case of unconstrained control input is within a finite band of asymptotically decaying error, the team concluded that MFC design leads to a stable controlled system, even when the control input is constrained.

To verify the stability equations experimentally, the MFC is designed to control the indoor air temperature in a building. The building HVAC model employed for simulations is given as:

$$
\begin{gathered}
x(k+1)=A x(k)+B u(k)+G w(k) \\
y(k)=C x(k)+D u(k) .
\end{gathered}
$$

Here, $u, y \in \mathbb{R}$ are the input to the building HVAC and indoor air temperature, respectively; $w \in \mathbb{R}^{2}$ is the disturbance; and $x \in \mathbb{R}$ is the state of the system. It is a first-order system, and the disturbances considered are external temperature and solar irradiance. MFC is employed to maintain the indoor air temperature at a desired set point. Simulations are performed for two cases: unconstrained control input and control input constrained, as in Eq. (A-46). Figure A-66 shows the error in indoor air temperature obtained in these two cases. The blue line represents the error obtained when the control input is unconstrained. Although the blue line is theoretically expected to be asymptotically decaying, according to Eq. (A-57), there is a fluctuating decay. This is a consequence of estimation error (i.e., $\phi \neq F$ ). However, the pink line, which corresponds to the error obtained when the control input is constrained, is within $\pm \frac{0.5 \alpha}{K_{p}}$ of the blue line. This band is marked by dotted red lines. The corresponding control effort in both the cases is shown in Figure A-67(a), and the obtained indoor air temperatures are shown in Figure A-67(b).

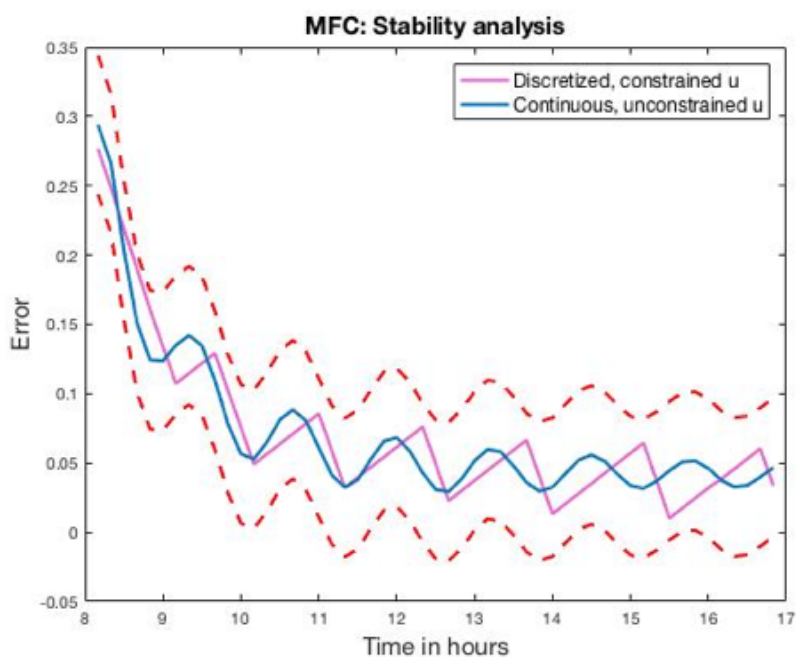

Figure A-66. Error in indoor air temperature for cases of unconstrained and constrained control input $\left({ }^{\circ} \mathrm{C}\right)$. 


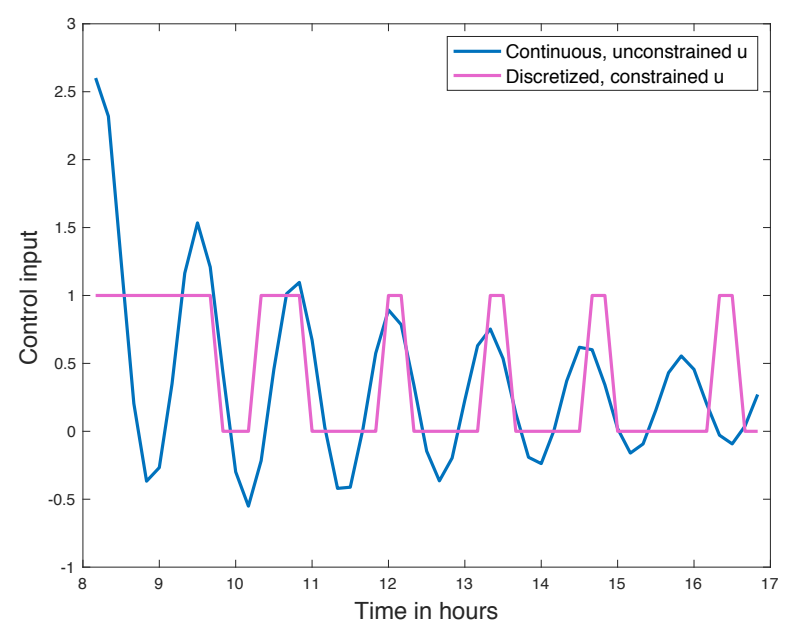

(a)

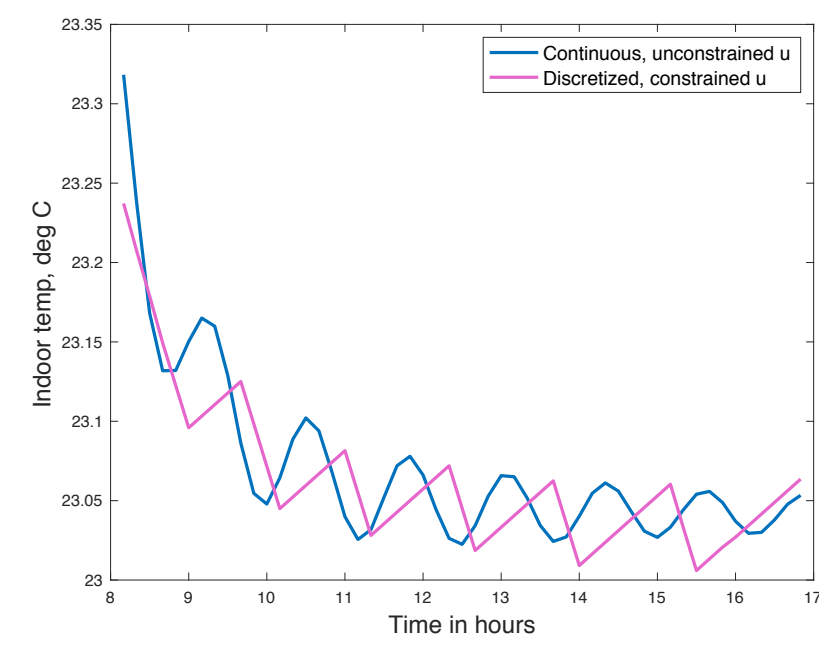

(b)

Figure A-67. (a) Control input for cases of unconstrained and constrained control input. (b) Indoor air temperature for cases of unconstrained and constrained control input.

\section{A.7.4 Generalization of MFC to Different Types of Loads}

The problem of allocating generated PV power to control the HVAC units of the same power load of $N$ number of buildings was handled with in the previous sections and was published in Telsang et al. [148] and Bara et al. [149]. This section considers a combination of residential and commercial buildings equipped with HVAC units of different power ratings. The precise problem considered is to allocate the generated solar PV power among residential and commercial buildings while maintaining the indoor temperatures near the desired set point.

The MFC methodology is employed to control the HVAC units. Because MFC generates a continuous value of the control input and an HVAC unit can be only on or off $(u=\{0,1\})$, the control input from MFC is rounded off and then fed to the HVAC unit. The details of implementation and stability constraints are described in the previous section and published in Telsang et al. [152]. Under this methodology, the obtained results are shown in Figures A-68 and A-69. The power rating is $2.25 \mathrm{~kW}$ for residential HVAC units and $4.5 \mathrm{~kW}$ for commercial HVAC units. In Figures A-68 and A-69, there was no PV power constraint on the amount of power that can be consumed by residential or commercial HVAC units. The power consumed is still shown as an additional insight into how much power would be "ideally required." 

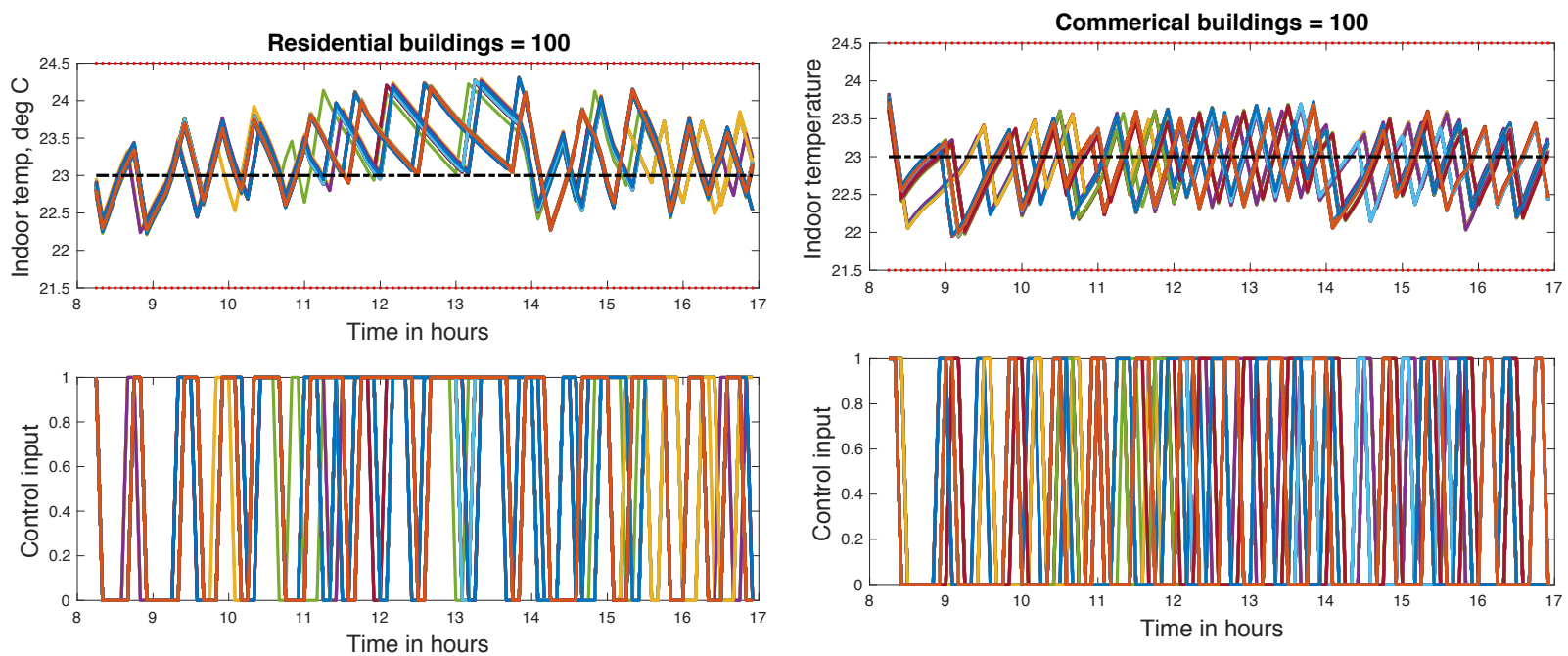

Figure A-68. Indoor temperatures and control inputs for 100 residential and 100 commercial buildings controlled by using MFC without PV power constraint.

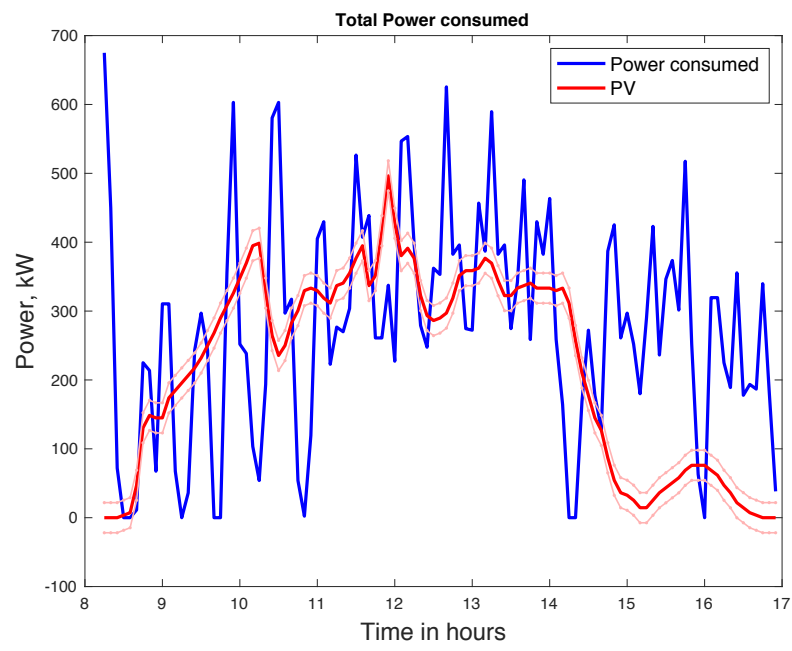

Figure A-69. Total power consumed by 100 residential and 100 commercial buildings controlled by using MFC without PV power constraint and compared with the generated PV power.

For simulation purposes, cooling with an HVAC unit is modeled as a first-order model with input $u=$ $\{0,1\}$, which is the state of the HVAC unit (i.e., on or off), and indoor temperature is modeled as the output. The external disturbances considered are external temperature, denoted $u_{e}$, and solar irradiation, denoted $u_{s}$.

$$
\begin{gathered}
\dot{x}(t)=A x(t)+B u(t)+G_{1} u_{s}+G_{2} u_{e} \\
y(t)=C x(t)+D u(t) .
\end{gathered}
$$

For a residential model, the state-space model parameters are:

$$
A=-1.1 \times 10^{-5}, B=-0.0011, G 1=3.4 \times 10^{-5}, G 2=0.0067, C=1, D=0 \text {, }
$$


whereas for a commercial model, the state-space model parameters are:

$$
A=-2.2 \times 10^{-5}, B=-0.0011, G 1=6.7 \times 10^{-5}, G 2=0.0134, C=1, D=0 .
$$

Let the number of residential loads that were turned on at time $t$ be $c_{1}(t)$ and the number of commercial loads that were turned on be $c_{2}(t)$. For the MFC results in Figure A-68, the indoor temperatures are within the permissible bounds, and these would be the "ideal" number of units that we would turn on if there were no constraints on the PV power consumed. However, there is a PV power constraint. At time $t$, let the amount of available (i.e., generated) PV power be $P V(t)$.

The constraint is to employ all of $P V(t)$ among the HVAC units of both the residential and commercial buildings while not straying from the desired set points. The definitions for some notations at time $t$ are provided as follows:

- Amount of available power: $P V$

- Power rating of a residential HVAC unit: $e_{r}$

- Power rating of a commercial HVAC unit: $e_{c}$

- Total number of residential HVAC units: $B l d_{r}$

- Total number of commercial HVAC units: $B_{l} d_{c}$

- Ideal number of residential HVAC units to be turned ON: $c_{1}$

- Ideal number of commercial HVAC units to be turned ON: $c_{2}$

- [Variable] Number of residential HVAC units to turn ON: $x_{1}$

- [Variable] Number of commercial HVAC units to turn ON: $x_{2}$

The power constraint can be mathematically expressed as:

$$
e_{r} x_{1}+e_{c} x_{2}=P V
$$

At a given time $t, P V, e_{r}$, and $e_{c}$ are constants. Hence, Eq. (A-62) represents a straight line, where $x_{1}$ is one dimension and $x_{2}$ is the second dimension. Because the ideal number of residential and commercial HVAC units to turn on is $\left(c_{1}, c_{2}\right)$ and the constraint is given by the straight line in Eq. (A-62), the optimal solution would be the orthogonal projection of $\left(c_{1}, c_{2}\right)$ onto the line in Eq. (A-62). This is shown in Figure A-70(a).

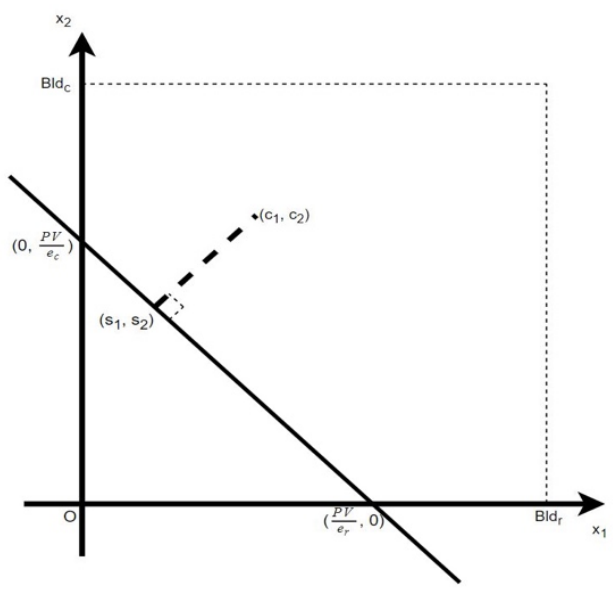

(a)

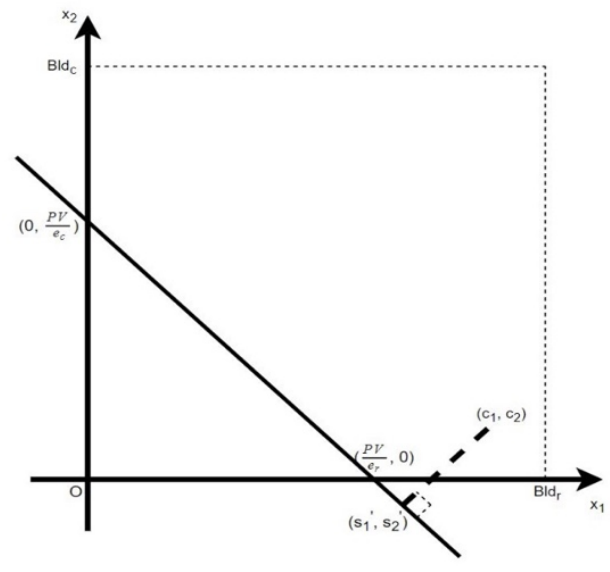

(b)

Figure A-70. Projection of the ideal number of residential and commercial HVAC units to turn on $\left(c_{1}, c_{2}\right)$ onto the constraint line in Eq. (A-62). 
The analytical solution of the orthogonal projection of $\left(c_{1}, c_{2}\right)$ onto the line in Eq. (A-62) is derived as follows. The coordinates of any point on the line in Eq. (A-62) are:

$$
\left(\frac{P V-e_{c} x_{2}}{e_{r}}, x_{2}\right)
$$

and the slope of the line in Eq. (A-62) is:

$$
m_{1}=-\frac{e_{r}}{e_{c}}
$$

So, the slope of a line connecting $\left(c_{1}, c_{2}\right)$ to any point in the line in Eq. (A-62) is described by:

$$
m_{2}=\frac{x_{2}-c_{2}}{\frac{P V-e_{c} x_{2}}{e_{r}}-c_{1}}
$$

Because the team was interested in the orthogonal projection of $\left(c_{1}, c_{2}\right)$ on the line in Eq. (A-62), the team considered the line orthogonal to the line in Eq. (A-62) that passes through $\left(c_{1}, c_{2}\right)$. Therefore, the team used of the property that the product of slopes of two orthogonal lines is -1 (i.e., $m_{1} . m_{2}=-1$ ). Substituting for the slopes gives:

$$
x_{2}=\frac{e_{c} P V-e_{c} e_{r} c_{1}+e_{r}^{2} c_{2}}{e_{r}^{2}+e_{c}^{2}}
$$

and substituting this in the line in Eq. (A-62) gives:

$$
x_{1}=\frac{e_{r}^{2} P V+e_{c}^{2} e_{r} c_{1}-e_{c} e_{r}^{2} c_{2}}{e_{r}\left(e_{r}^{2}+e_{c}^{2}\right)}
$$

Equations (A-66) and (A-67) yield the optimal solution for the number of HVAC units to be turned on in commercial and residential buildings, respectively. The optimal solution is denoted by $\left(s_{1}, s_{2}\right)$.

Additional constraints on $\left(x_{1}, x_{2}\right)$ are:

$$
\begin{aligned}
& 0 \leq x_{1} \leq B l d_{r} \\
& 0 \leq x_{2} \leq B l d_{c}
\end{aligned}
$$

An example of the cases in which the optimal solution violates these constraints is shown in Figure A-70(b). The optimal case is denoted by $\left(s_{1}^{\prime}, s_{2}^{\prime}\right)$. However, $s_{2}^{\prime}<0$, which violates the constraint in Eq. (A-69). In such a case, the nearest solution that does not violate constraints in Eqs. (A-68) and (A-69) would be chosen. In this case, it would be $x_{1}=s_{1}^{\prime}, x_{2}=0$.

The final solution (optimal or near optimal) denoted by $\left(s_{1}, s_{2}\right)$ will be the final number of HVAC units that will be turned on at time $t$ in residential and commercial buildings. Given $s_{1}$, the $s_{1}$ number of buildings with the highest control input value as recommended by MFC will be turned on. Similarly, the $s_{2}$ number of commercial units are turned on.

For simulation purposes, the following values were considered: $e_{r}=2.25 \mathrm{~kW}, e_{c}=4.5 \mathrm{~kW}$, and $B l d_{r}=$ $B l d_{c}=100$. The desired set point for temperature is $23^{\circ} \mathrm{C}$, and the permissible band is $\pm 1.5^{\circ} \mathrm{C}$. The results obtained with the developed MFC methodology for handling the power constraint are presented in Figure A-71. After 3:00 p.m., the problem is infeasible because there is not enough generated PV power to support the cooling of all the buildings within the desired temperature bands. Moreover, the ideal 
available power is between 10:00 a.m. and 2:00 p.m. wherein both the temperature and power constraints are met. Because the power available is insufficient after 3:00 p.m., PV power constraint is not implemented after 3:00 p.m. The results obtained when the PV constraint is implemented until 3:00 p.m. and turned off after 3:00 p.m. are shown in Figure A-72.
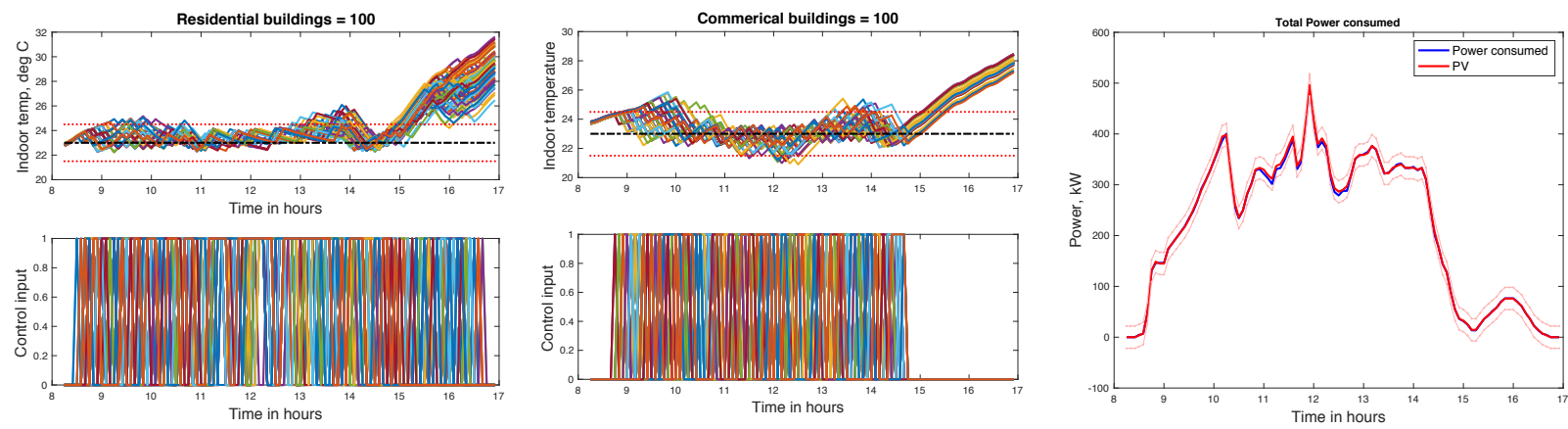

Figure A-71. Indoor temperatures, control inputs, and total power consumed for 100 residential and 100 commercial buildings controlled by using MFC with PV power constraint.
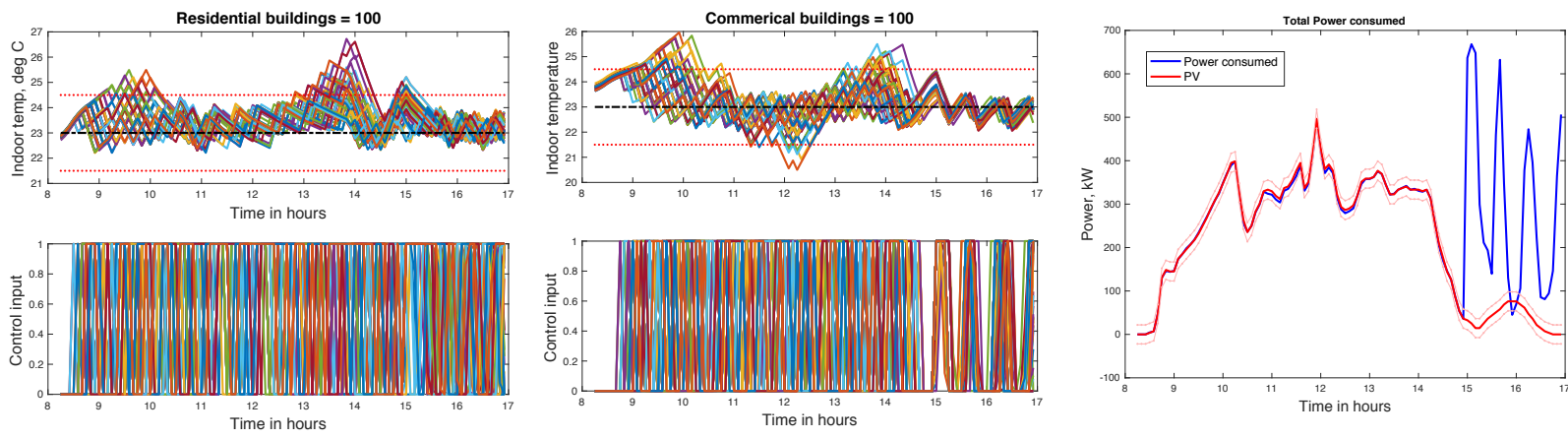

Figure A-72. Indoor temperatures, control inputs, and total power consumed for 100 residential and 100 commercial buildings controlled by using MFC with PV power constraint.

In the previous section, the power consumption of all the buildings was restricted to the available power. However, as seen in the simulation results, although the power constraint was impeccable, there were considerable indoor temperature deviations from the comfort band. Therefore, the power constraint can be relaxed as follows. The power consumed should be within a threshold band of the available power. Denote the threshold as $\epsilon$. Then, the relaxed PV power constraint is that the power consumed must be within $\pm \epsilon$ of the available power. Mathematically, the power constraint in Eq. (A-62) will be relaxed as:

$$
\begin{gathered}
P V-\epsilon \leq e_{r} x_{1}+e_{c} x_{2} \leq P V+\epsilon, \\
e_{r} x_{1}+e_{c} x_{2}=P V+\epsilon, \\
e_{r} x_{1}+e_{c} x_{2}=P V-\epsilon .
\end{gathered}
$$

This relaxation is implemented by finding the distance of $\left(c_{1}, c_{2}\right)$ to the lines in Eqs. (A-62), (A-71), and (72). Then, the line that corresponds to the shortest of the three distances will be closest to $\left(c_{1}, c_{2}\right)$, and hence the orthogonal projection of $\left(c_{1}, c_{2}\right)$ onto that line will be the final solution. The results under the relaxed PV constraint are shown in Figure A-73. Moreover, because the power available is insufficient 
after 3:00 p.m., PV constraint is not implemented after 3:00 p.m. The temperature deviations under relaxed PV constraint are lesser than the results obtained under strict PV constraint. Also, the power consumed stays within the threshold of the available power.
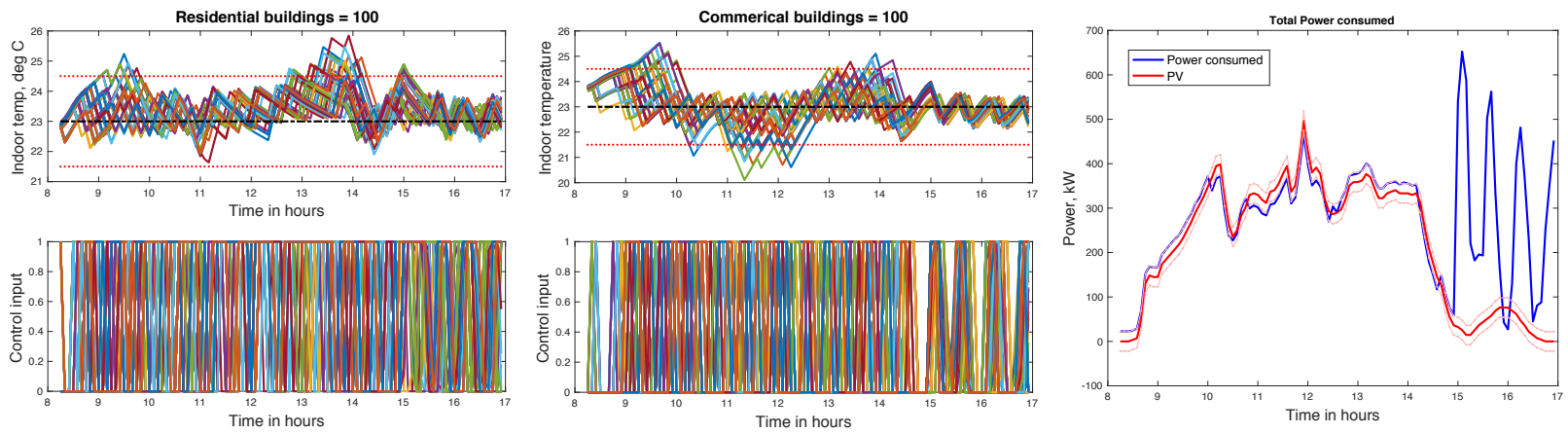

Figure A-73. Indoor temperatures, control inputs, and total power consumed for 100 residential and 100 commercial buildings controlled by using MFC with PV power constraint.

Next, the team considered the MFC problem under the scenario in which one kind of load should be prioritized over another. For example, if there is a constraint that during afternoons, it is more important for commercial building indoor temperatures to be within the temperature comfort band than it is for indoor temperatures in residential buildings, the team would assign a higher weight to commercial loads. Let the weight of the commercial load be $w \in[0,1]$, where a higher value of $w$ implies more importance on the commercial load and correspondingly less importance on the residential load. That is, the weight on the residential load would be $1-w$. For a given weight $w$, this problem is solved by using following steps (Figure A-74).

1. Determine the corresponding angle of projection from $\theta=f(w)$.

2. Find the points $\left(x_{0}, y_{0}\right)$ on the power constraint line so that the angle between the line segment connecting $\left(x_{0}, y_{0}\right)$ and $\left(c_{1}, c_{2}\right)$ and the power constraint line is $\theta$.

3. The coordinate $\left(x_{0}, y_{0}\right)$ would be optimal solution for a given weight. 


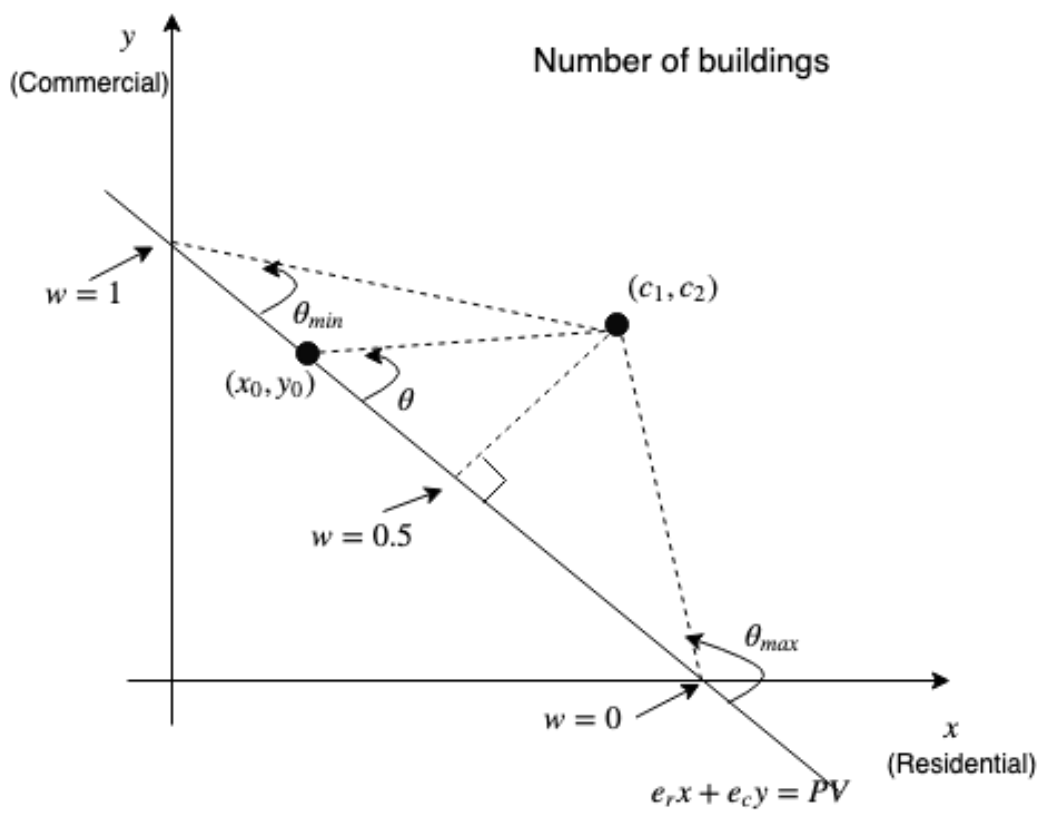

Figure A-74. Mapping importance weight to projection angle.

Consider a piecewise-continuous function $f:[0,1] \rightarrow\left[\theta_{\min }, \theta_{\max }\right]$ so that:

$$
f(0)=\theta_{\max }, f\left(\frac{1}{2}\right)=\frac{\pi}{2}, f(1)=\theta_{\min }
$$

Here, $\theta_{\min }$ corresponds to the projection angle in the case of maximum importance on the indoor temperatures of commercial buildings, and $\theta_{\max }$ corresponds to the case of least importance on commercial loads.

For a given time $t$, for a computed $\left(c_{1}, c_{2}\right)$, and the power constraint line $e_{r} x+e_{c} y=P V, \theta_{\min }$ and $\theta_{\text {max }}$ are shown in Figure A-74. The function $f$ is defined as:

$$
f(w)= \begin{cases}\left(\pi-2 \theta_{\max }\right) w+\theta_{\max }, & \text { if } w \in[0,0.5] \\ \left(2 \theta_{\min }-\pi\right) w-\theta_{\min }+p i, & \text { if } w \in[0.5,1]\end{cases}
$$

Let $\theta_{1}$ be the angle made by the constraint line with $x$-axis. Then:

$$
\tan \theta_{1}=-\frac{e_{r}}{e_{c}}
$$

Denote the line segment joining any point $(x, y)$ on the constraint line and the point $\left(c_{1}, c_{2}\right)$ by $l(x, y)$. Let $\theta_{2}$ be the angle made by $l$ with the $x$-axis. Correspondingly, let $\theta_{2 \min }$ be the angle between $l\left(0, P V / e_{c}\right)$ and the $x$-axis, and let $\theta_{2 \max }$ be the angle between $l\left(P V / e_{r}, 0\right)$ and the $x$-axis. Ideally, the required power would be $P V_{r e q}=c_{1} e_{r}+c_{2} e_{c}$. Depending on whether $P V_{r e q}-P V$ is positive or negative, the point $\left(c_{1}, c_{2}\right)$ will be above or below the line. Moreover, the wrapping of angles poses additional difficulty. Therefore, accounting for all possible scenarios, $\theta_{\min }$ and $\theta_{\max }$ are calculated as follows:

$$
\theta_{\text {min }}=\left|\theta_{1}-\theta_{2 \min }\right| .
$$


Case 1: $P V_{\text {req }}-P V \geq 0$ :

$$
\begin{gathered}
\theta_{\max }=\left\{\begin{array}{c}
\left|\theta_{1}-\theta_{2 \max }\right|, \text { if } \theta_{2 \max }>0 \\
|\pi-| \theta_{2 \max }\left|-\theta_{1}\right|, \text { if } \theta_{2 \max }<0
\end{array}\right. \\
\theta=f(w), \\
m=\tan \left(\theta+\theta_{1}\right) .
\end{gathered}
$$

Case 2: $P V_{\text {req }}-P V<0$ :

$$
\begin{gathered}
\theta_{\max }=\left|\theta_{1}-\theta_{2 \max }\right|, \\
\theta=f(w), \\
m=\tan \left(\theta_{1}-\theta\right) .
\end{gathered}
$$

Then, the coordinates $x_{0}, y_{0}$ are computed as follows:

$$
\begin{gathered}
x_{0}=\frac{e_{c}\left(c_{1} m-c_{2}\right)+P V}{e_{r}+e_{c} m} \\
y_{0}=\frac{P V-e_{r} x_{0}}{e_{c}}
\end{gathered}
$$

Simulations are carried with the developed strategy for 100 commercial and residential buildings each for a given generated power profile. Results are shown in Figures A-75 through A-77 for three different cases of weights: $w=0.15,0.5$, and 0.9 , respectively. Note that $w=0.5$ corresponds to equal importance on both commercial and residential loads, corresponding to the orthogonal projection of the point $\left(c_{1}, c_{2}\right)$ on the constraint line. The strategy is not implemented after 3:00 p.m. because the available power is much lesser than required, making the solution infeasible. In each of the three simulation cases of different weights, the consumed power is within the threshold of that available. On the other hand, the indoor temperatures of residential and commercial buildings stay within or deviate from the comfort band, depending on the applied importance. 

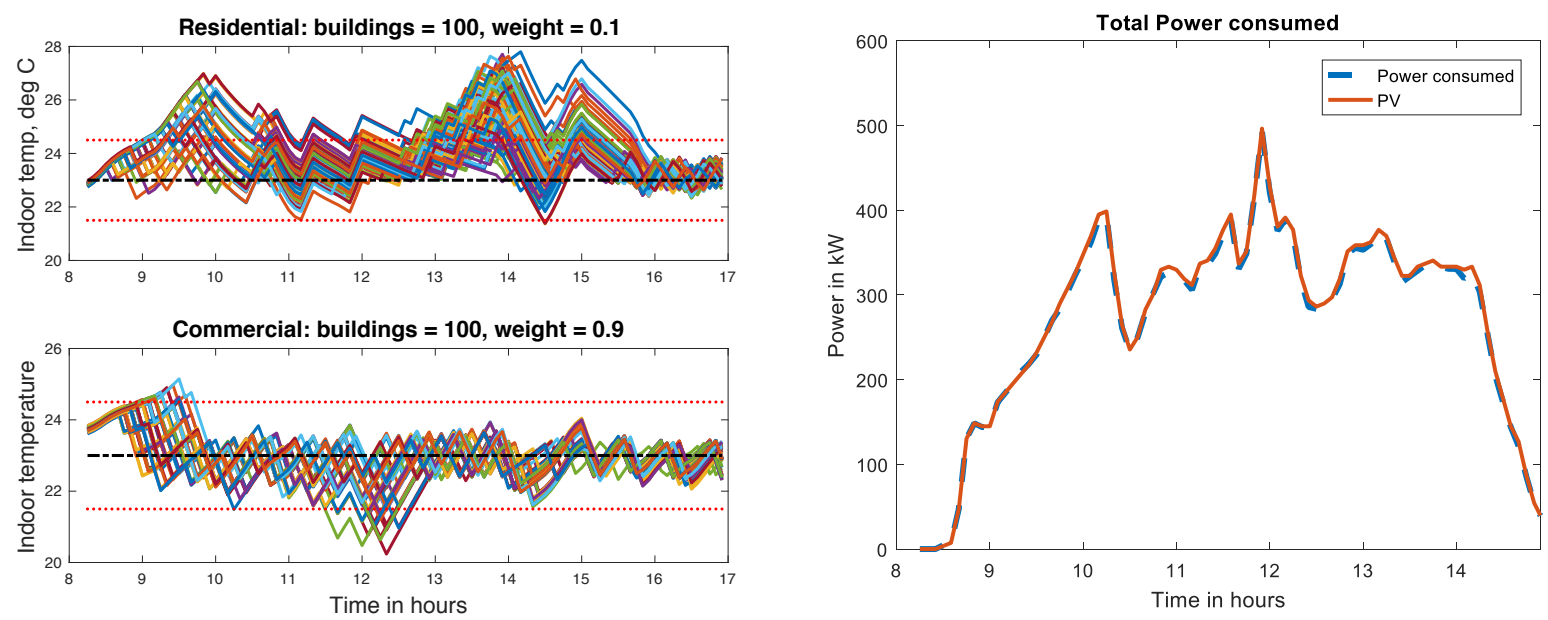

Figure A-75. Indoor temperatures and total power consumed for 100 residential and 100 commercial buildings controlled by using MFC with PV power constraint $(w=0.9)$.
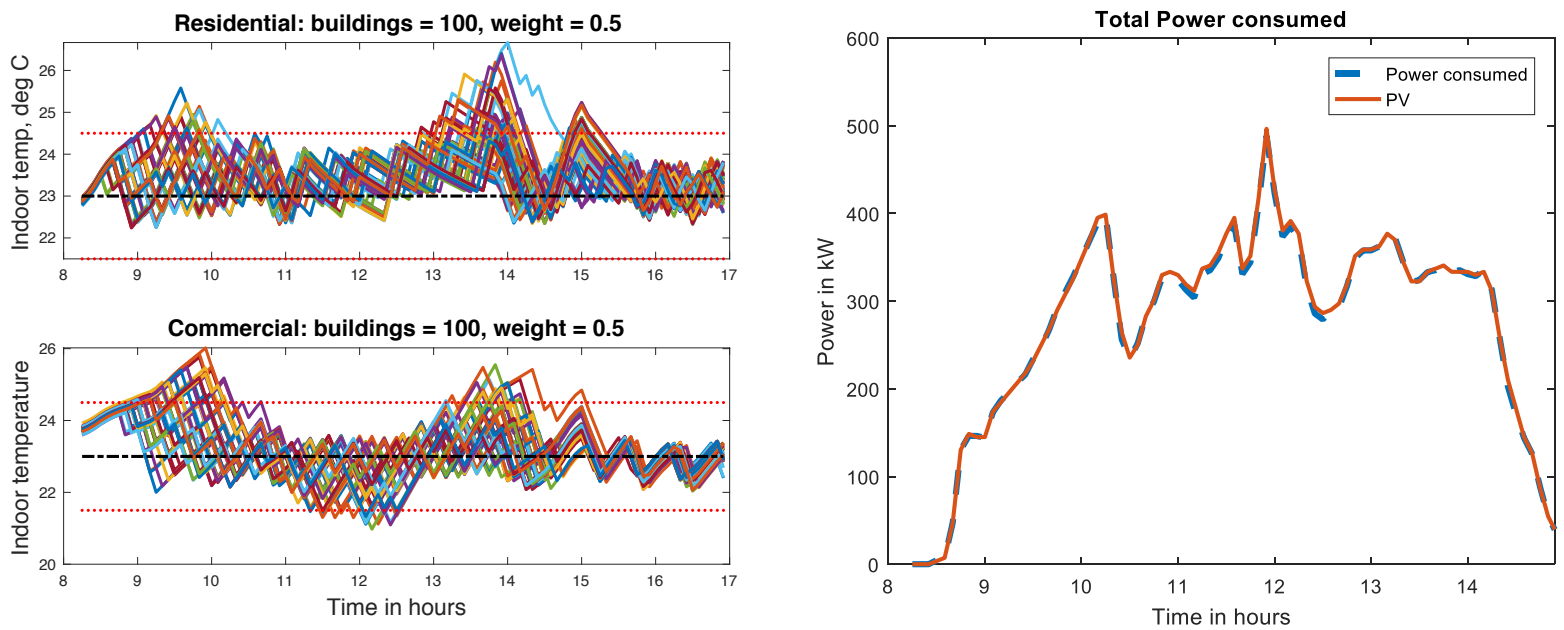

Figure A-76. Indoor temperatures and total power consumed for 100 residential and 100 commercial buildings controlled by using MFC with PV power constraint $(w=0.5)$. 

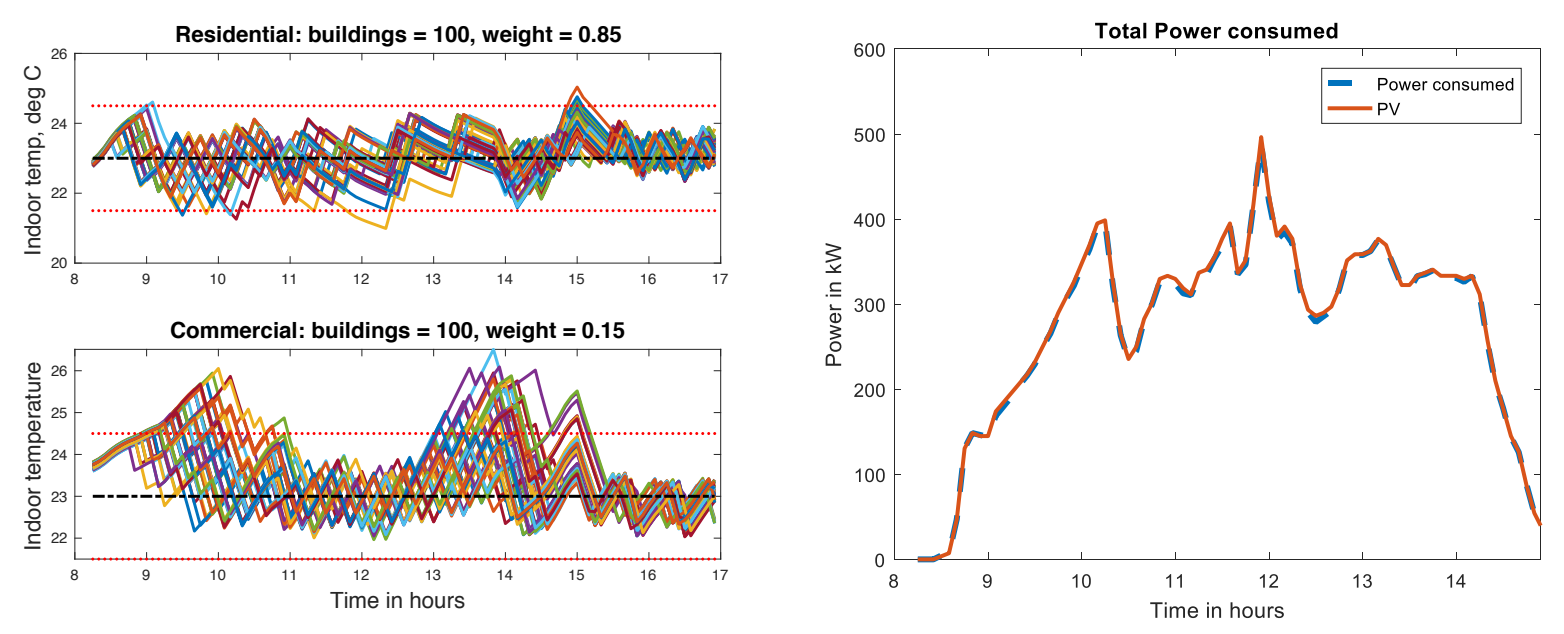

Figure A-77. Indoor temperatures and total power consumed for 100 residential and 100 commercial buildings controlled by using MFC with PV power constraint $(w=0.15)$.

Then, the team extended the problem to multiple loads (not necessary two different loads), each with different importance (i.e., priorities). The theoretical derivations are extended to $n$ types of loads. Simulation results for three different types of loads are presented next.

Simulations were carried with the developed strategy for 100 commercial HVAC units, 100 residential HVAC units, and $100 \mathrm{WHs}$ for a given generated PV power profile. Figure A-78 shows indoor temperatures and total power consumed for 400 residential HVAC units split into four sets of equal importance/priorities. Figure A-79 shows the results for the same scenario but with different importance/priorities. The indoor temperatures for a few of the100 HVAC units with lesser priority $(w=$ 0 ) deviated outside the comfort band for some time, whereas the indoor temperatures for the100 HVAC units with higher priority $(w=0.5)$ became closer to the desired temperature set point.
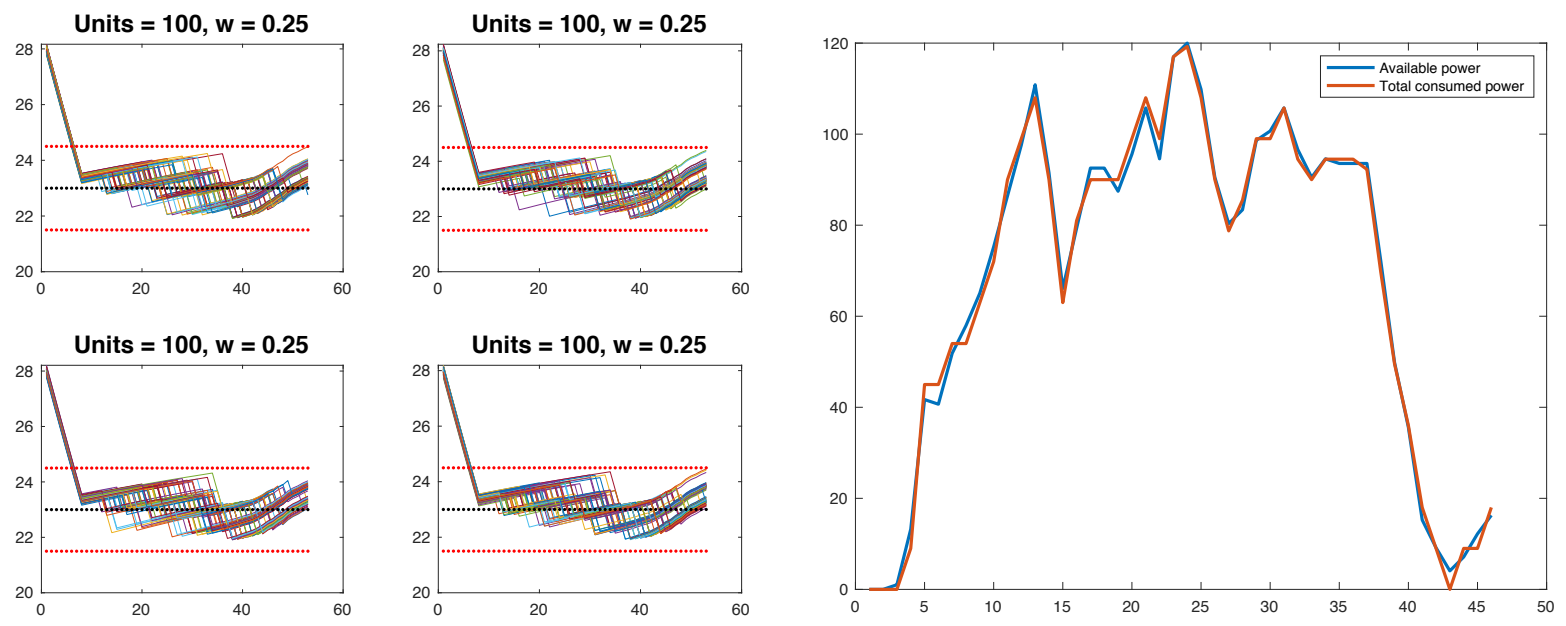

Figure A-78. Indoor temperatures and total power consumed for 400 residential HVAC units controlled by using MFC with equal priorities and PV power constraint. 

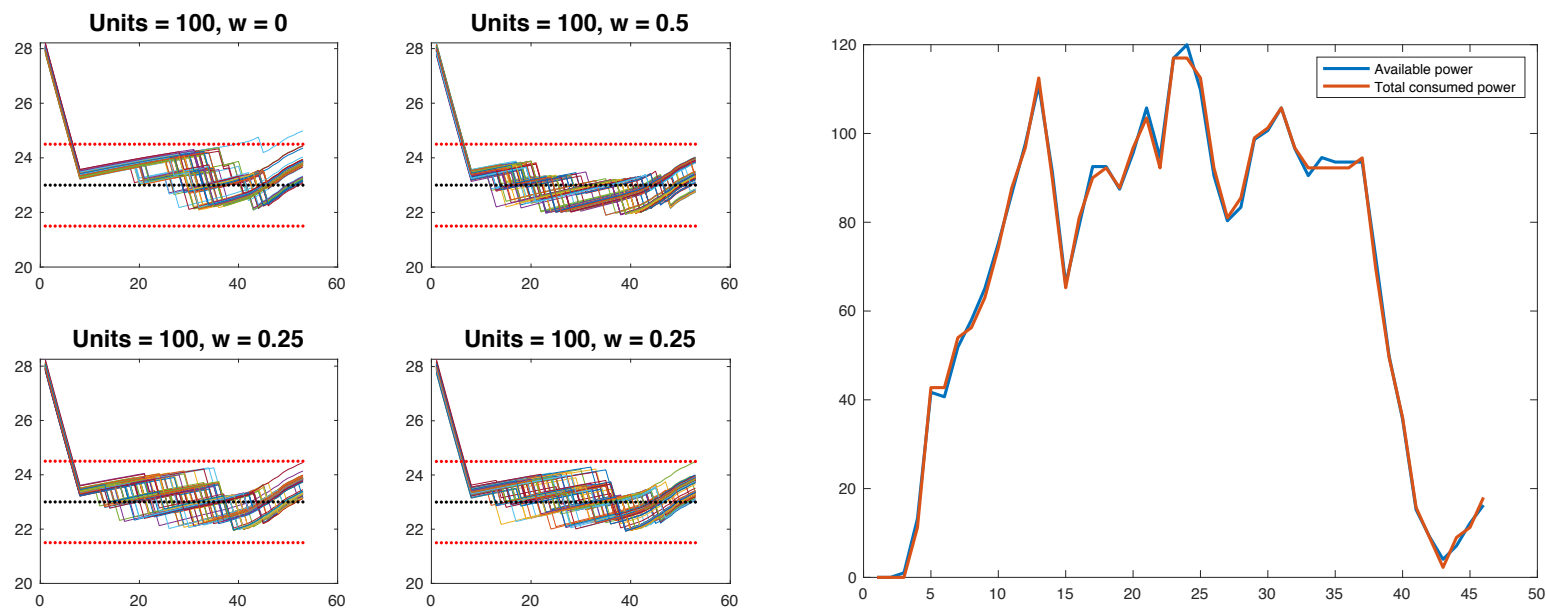

Figure A-79. Indoor temperatures and total power consumed for 400 residential HVAC units controlled by using MFC with different priorities and PV power constraint.

Figure A-80 shows the indoor temperatures and total power consumed for 100 residential HVAC units, 100 commercial HVAC units, and 100 WHs controlled by using MFC with equal priorities and PV power constraint. Indoor temperatures for residential and commercial buildings went out of the comfort band for some time. To provide higher priority for residential buildings, the weight for residential buildings was increased to $w=1$ and, as shown in Figure A-81, indoor temperatures for such buildings have always become within the comfort band. But to provide higher priority for commercial buildings, the weight for commercial buildings was increased to $w=1$ and, as shown in Figure A-82, indoor temperatures for such buildings have always been within the comfort band.

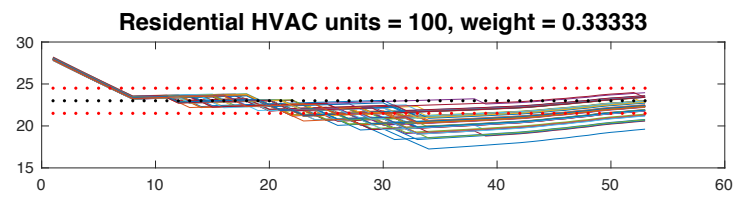

Water heater units $=100$, weight $=0.33333$

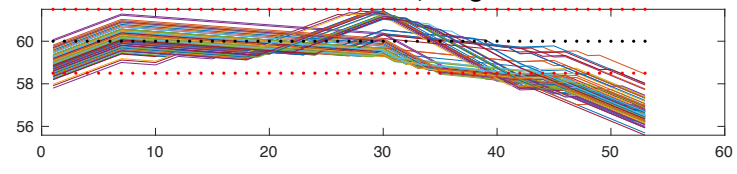

Commercial HVAC units $=100$, weight $=0.33333$
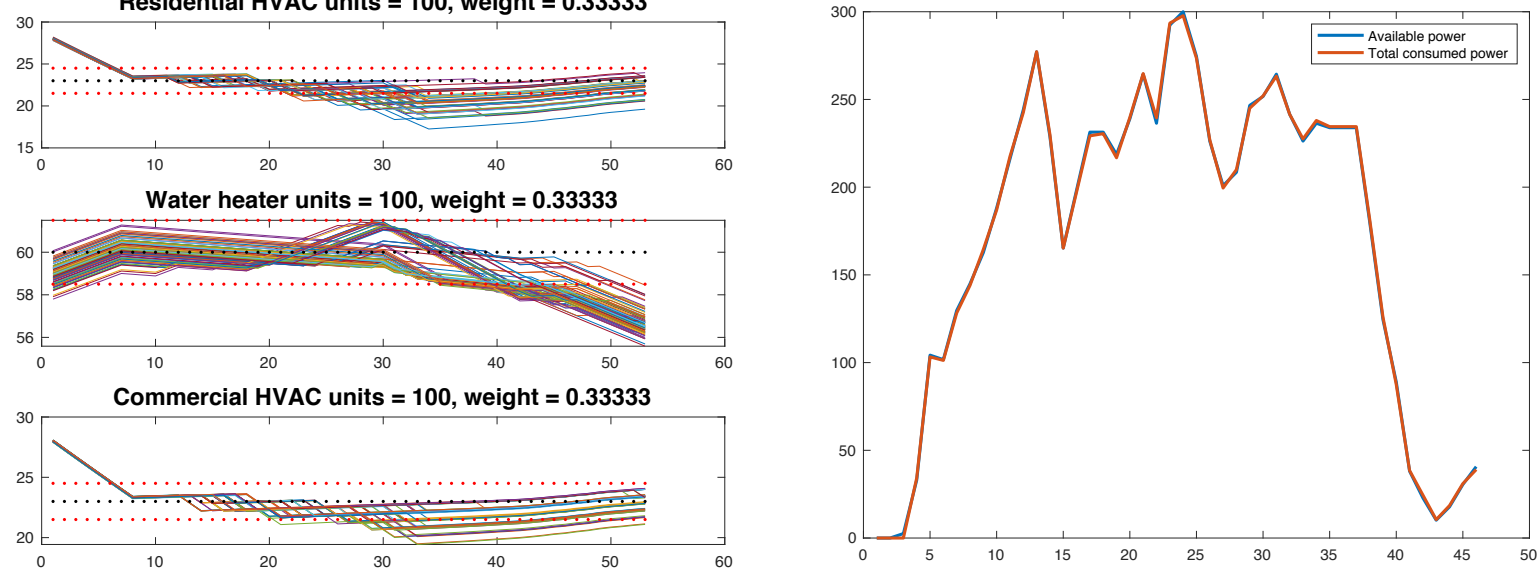

Figure A-80. Indoor temperatures and total power consumed for 100 residential HVAC units, 100 commercial HVAC units, and 100 WHs controlled by using MFC with equal priorities and PV power constraint. 

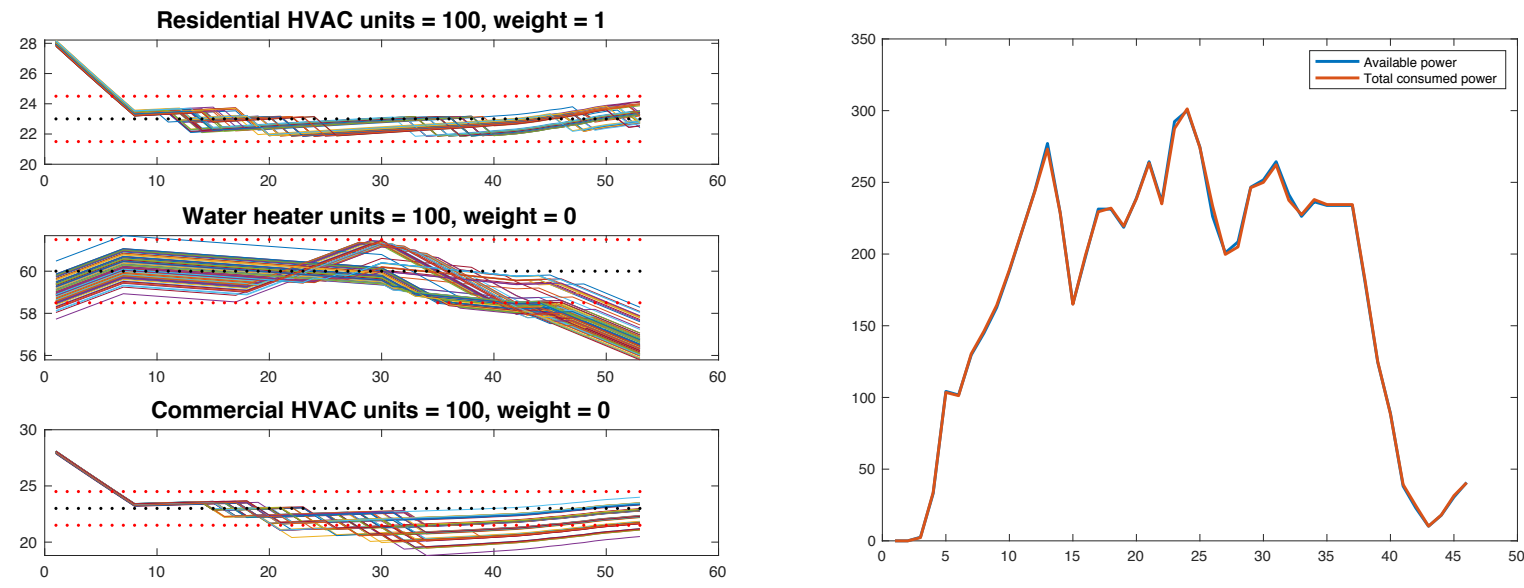

Figure A-81. Indoor temperatures and total power consumed for 100 residential HVAC units, 100 commercial HVAC units, and 100 WHs controlled by using MFC with different priorities and PV power constraint.
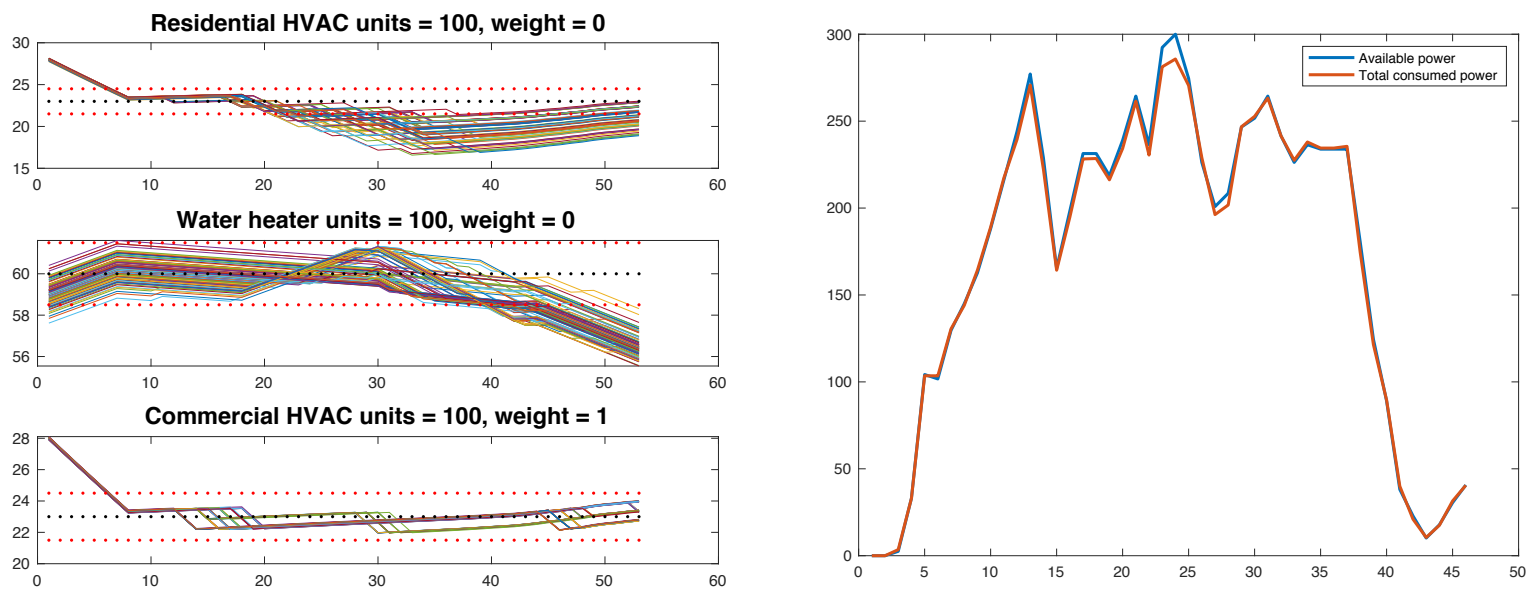

Figure A-82. Indoor temperatures and total power consumed for 100 residential HVAC units, 100 commercial HVAC units, and 100 WHs controlled by using MFC with different priorities and PV power constraint.

\section{A.8 DETAILS ON CONTROLLER DEPLOYMENT}

The current controller is a centralized Python3 program. The endpoints are the Ecobee thermostats mounted to the wall of each zone. The controller has five different parts.

1. Main controller loop: This coordinates the other modules and controls the timing of all data gathering and decisions. It also manages the API credentials for the Ecobee API. Outdoor weather data are also gathered by this module

2. State measurement modules: These are internal representations of the Ecobee thermostats. They query the state and temperature data and enforce the controller decisions on the Ecobee thermostats. The 
temperature data are queried every $3 \mathrm{~min}$, and the data are smoothed for system identification and forecasting.

3. Forecasting module: ARIMA forecasting is used to predict the temperature and PV input to be used in computing the optimal control.

4. System identification module: This module is responsible for tracking histories and computing the state matrices used by the optimization function. This is done by solving an LS regression.

5. Optimization module: This module is responsible for computing the optimal control. A mixed integer linear programming problem is solved via branch-and-cut to calculate the control over the control horizon $(1 \mathrm{~h})$. The optimization is implemented by using the COIN-OR solver and the Pulp Python interface.

Additionally, all data input and control output are logged into a MySQL historian. The controller architecture is shown in Figure . One priority for the current MPC controller was to move to a cloudbased application. This eased deployments and system status monitoring as opposed to on-site hardware deployments. The cloud infrastructure used by the ORNL team is AWS. In AWS, the controller will be deployed on a virtual machine via an EC2 instance. Because the control endpoints are Ecobee controllers accessed through the Ecobee API, the virtual machine will have no difficulty in reaching these points if the Ecobee controllers maintain an internet connection.

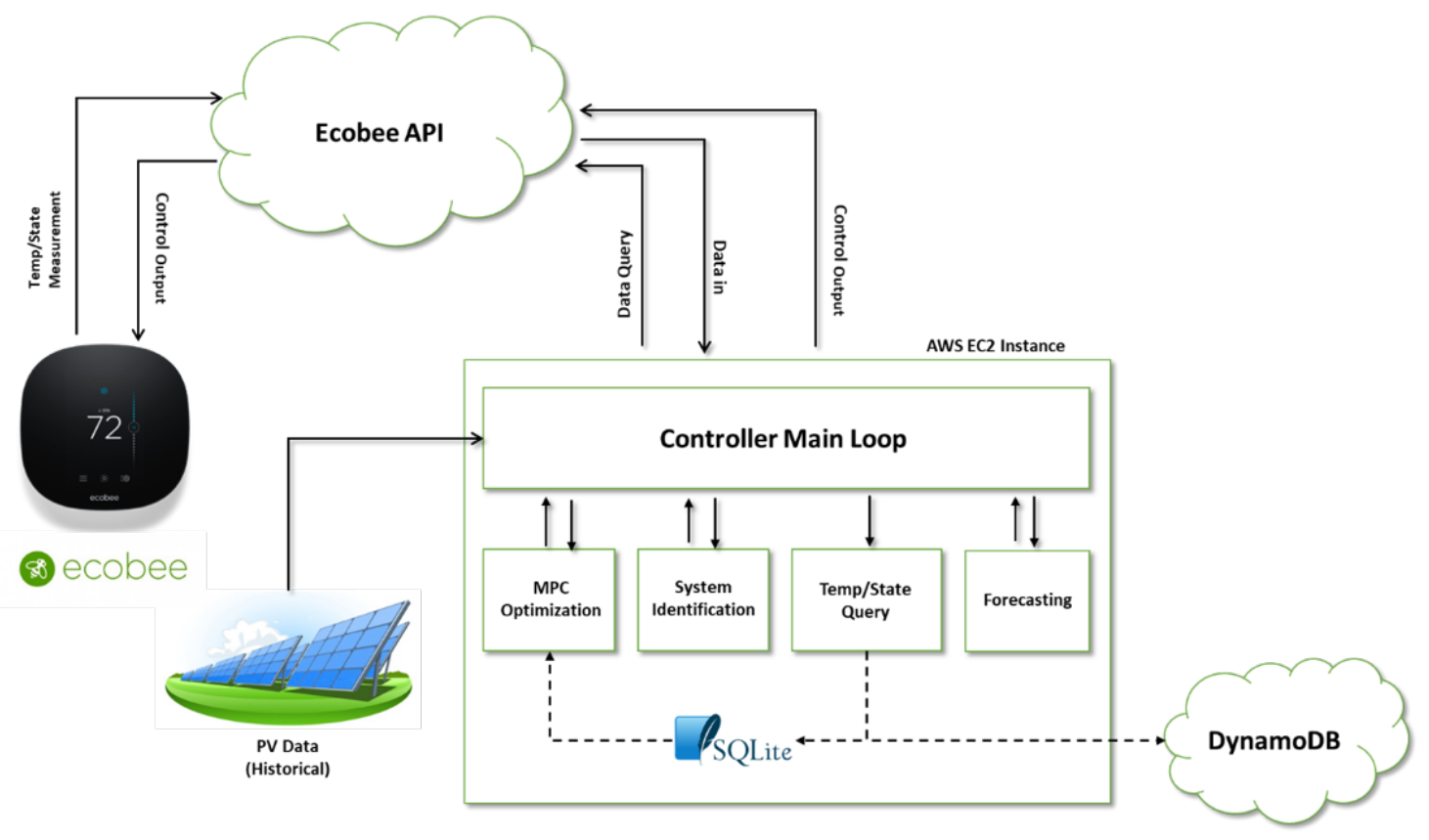

Figure A-83. Control architecture.

The fundamental controller deployment will remain the same, using a Python application with components responsible for gathering endpoint data, performing forecasting data forecasting, and solving the optimization problem. Aside from being hosted on an Amazon EC2 instance, an additional change is that data gathering is linked to the optimization computation through a local, short-time line SQLite database. This database easily allows for the validation and preprocessing of data before the control computation. These measurements are also linked to the AWS service DynamoDB, in which they are held for a longer period of time. 
In addition to moving to the cloud deployment, various features were added, such as better data preprocessing, to assist with forecasting solar data and system identification. In the future, live PV measurements will be implemented for the PV gathering. However, this measurement is currently taken from historical measurements. This offers a consistent baseline to test the control against. All application components can be easily transferred to any single-board computer, acting as a local site controller, such as a Raspberry Pi or Intel Nuc. Alternatively, the control is operated from a cloud infrastructure, such as an Amazon EC2 instance.

Because control actions are only taken once every 10-15 min, there is an interval of time within which the controller is only passively monitoring the endpoints but has no mechanism to compile an image of the system at large or take corrective action. The development team investigated ways in which a system monitoring process can be put in place. This included hard boundaries to ensure that the building's comfort boundaries are being enforced outside the controller and monitoring grid state to take unexpected corrective action in the event of a sudden change in that state.

Another development is that the data gathering component and optimization component were formerly separated in execution and are now linked by an underlying relational database. Previously, this relationship was a dependency in the execution. That is, the data management component gathered the data and passed it to the optimization module. If an externality, such as a network error, interrupts the first stage in this process, then optimization success will be less reliable. By using an application database, these two stages can be unlinked temporally. The data management module is responsible for gathering data, storing the data, and dealing with any external disruptions. The optimization module can then be responsible for interpreting the data; performing any preprocessing required, such as interpolation or extrapolation; and computing the control. The control can then be passed back to data management for dispatch to the devices; this relationship is illustrated in Figure A-84. This controller is written as a Python application with an underlying data basis layer for managing traffic. The controller can interface with several commercial smart devices but has been primarily tested with the Ecobee smart thermostat. For smooth deployment, the application is being containerized and will be deployable through Docker or a distributed production deployment service, such as Kubernetes. Testing has shown this to be a suitable deployment architecture, supporting the reliability and compartmentalization of the software components.

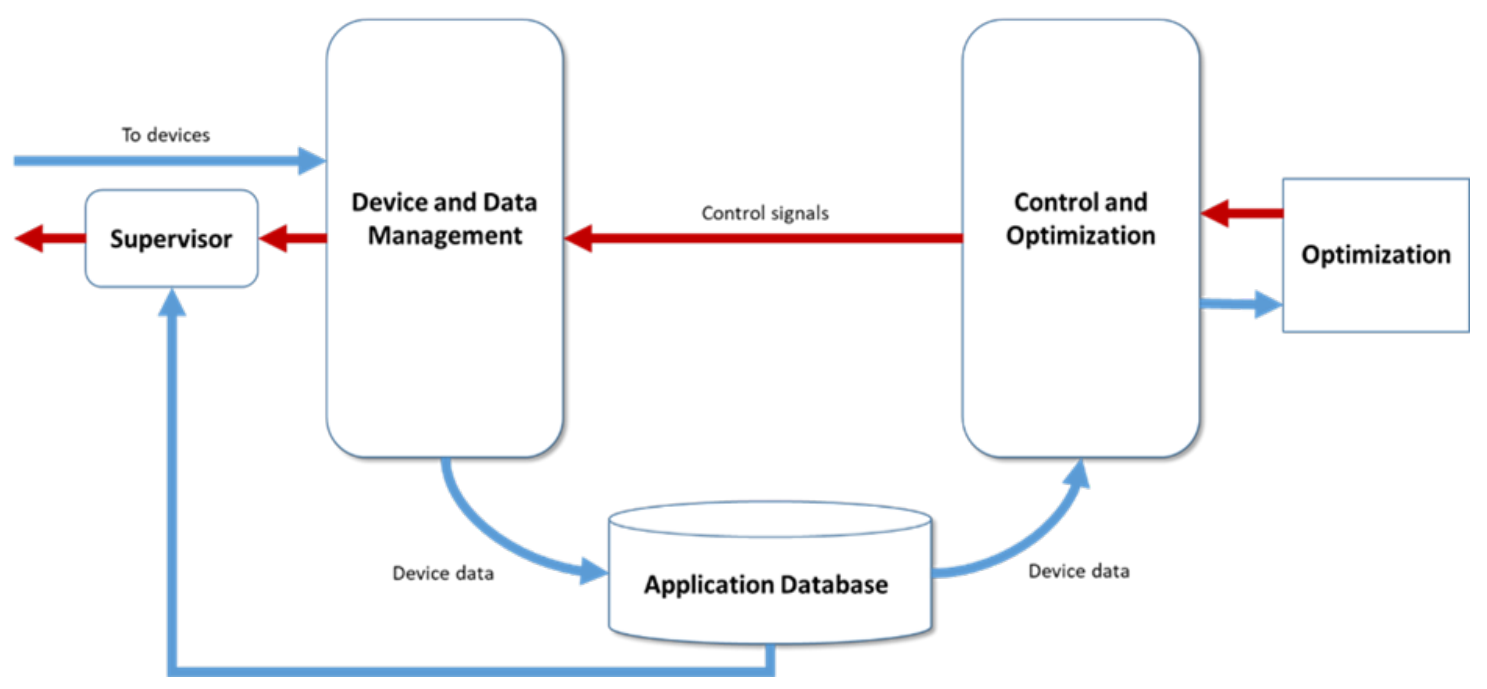

Figure A-84. Device/data management and optimization linked by the database. 
The final system architecture is shown in Figure A-84. Five distinct modules exist: the device and data management, the control and optimization, the optimization module, the application database, and the supervisor.

The device and data management module is responsible for interfacing with the devices that control the HVAC units. Primarily, this has been Ecobee smart thermostats, but the system is modular enough to allow interfaces to other devices if those interfaces conform to the standard of having several required methods. Examples of these methods are get_temp and set_state. The actual implementation of these methods is left to the deployment considerations.

Once the application starts, the system creates virtualized objects that represent the thermostats and then creates a process by which the physical devices are polled through the interfaces at regular intervals. The gathered data are then deposited into the application database. Additionally, the poll process checks for scheduled control actions and dispatches to the physical devices, if necessary.

The control and optimization module is responsible for coordinating the control process at each control period. The control process is a separate process from the device polling and works at a different timescale. At the start of each control period, the control module pulls in required data from the application databases, such as state information and environmental data, then performs system identification and forecasting, and finally calls the optimization module to compute the control. This flow is illustrated in Figure A-85.

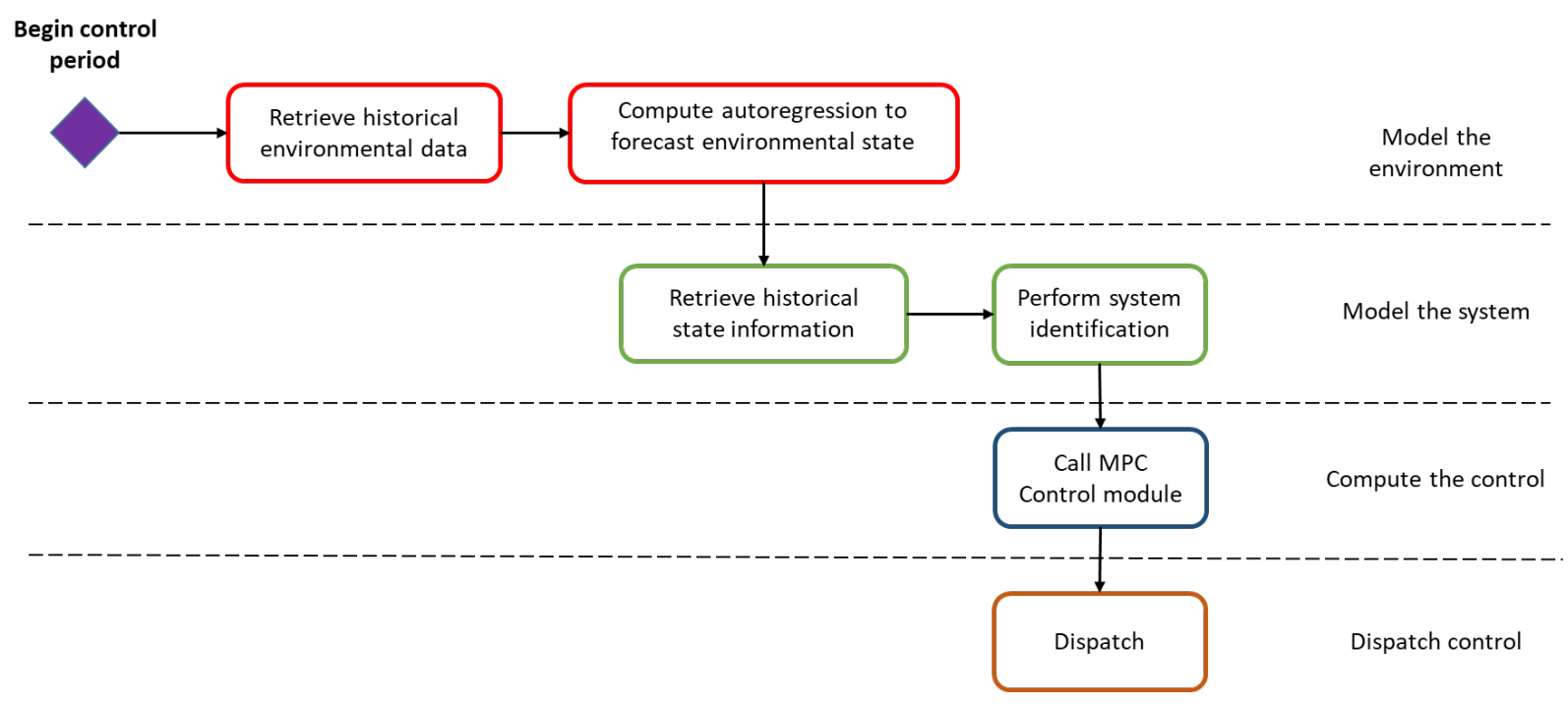

Figure A-85. Control flow.

The optimization module is the code that directly computes the optimal control by using the data aggregated by the control module. The optimization is written by using the Pulp Python interface and connects to the cut-and-branch COIN-OR solver. This is an open-source solver. Although restricted to a subclass of convex problems, the solver does not require the expensive license subscriptions that commercial solvers require. However, if a more complex optimization is formulated, then the new solver can be interchanged.

The application database is an SQL database and accessible to all code modules. Historical data are stored here, and older data are purged from the system at intervals. Including the database allows for the asynchronous operation of the control and data gathering processes because the control module can examine and validate the data in the database at its chosen time to make the correct control decisions. 
Breaking the temporal dependency of the control on data gathering has led to a more robust and resilient system in controller testing. The database is also fully interchangeable, depending on the deployment requirements.

The supervisor module sits outside the framework of the Sunlamp controller and exists to ensure that the controlled environment remains stable in the face of inaccurate data or unpredicted behavior. That is, if the building moves outside acceptable comfort boundaries, then the supervisor will step in to force the system back to comfort boundaries and then relinquish control back to the MPC controller.

The final workflow comprises three main stages: controller configuration, data gathering, and control computation and dispatch. This system timeline is described in more detail in Figure A- 86.

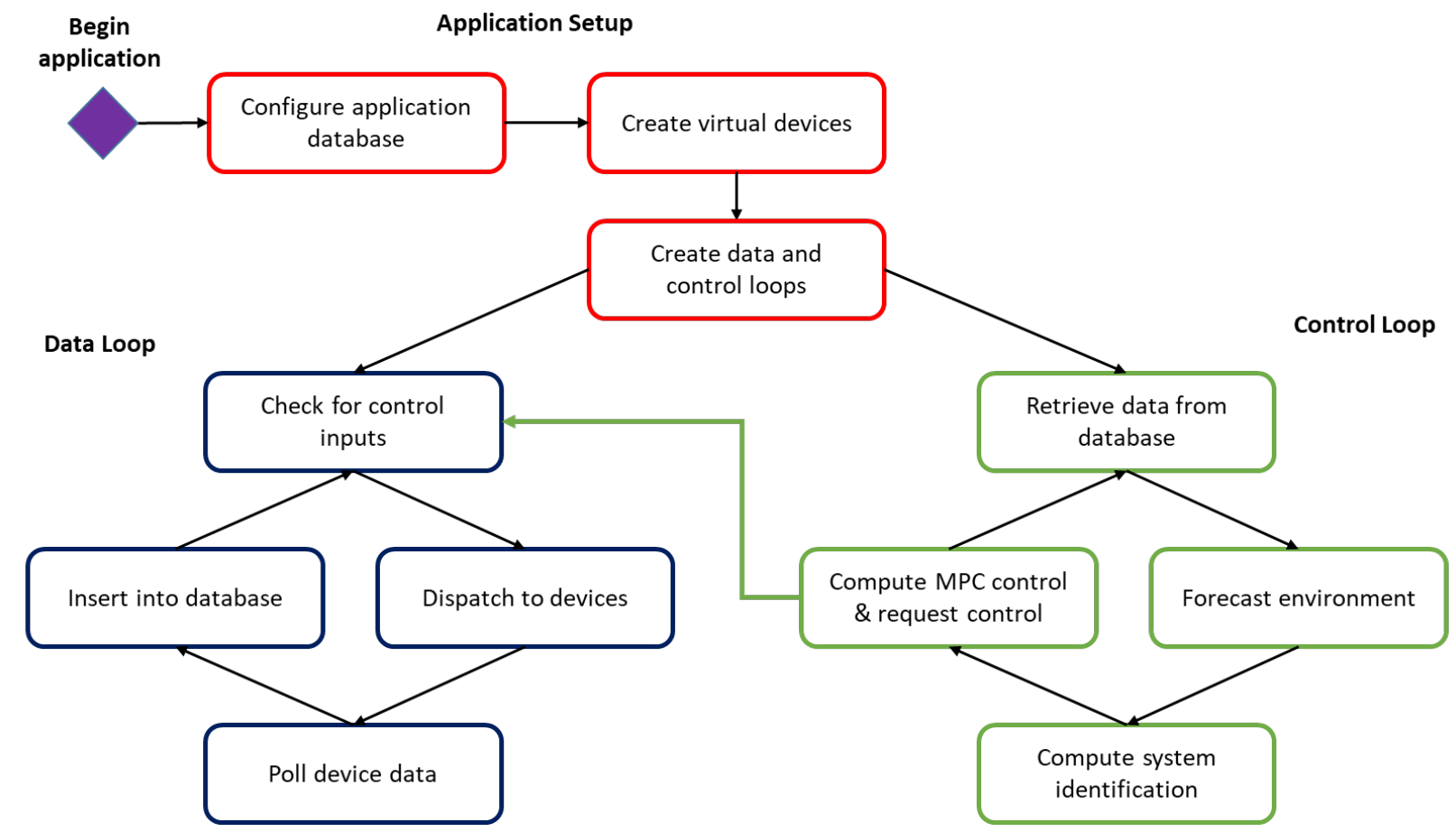

Figure A-86. Application flow diagram.

The final software package comprises 2,235 lines of Python code, not including any additional code required to deploy the software into different environments. For distribution, this code will be uploaded to a public code repository, such as GitHub or GitLab. In the future, testing will continue while weather is favorable alongside incremental improvements to any software bugs that arise.

Control periods occur every 12 min and comprise a data-gathering phase, computing the control and then enforcing the control decisions. The control workflow can be seen in Figures A-87 and A-88. 


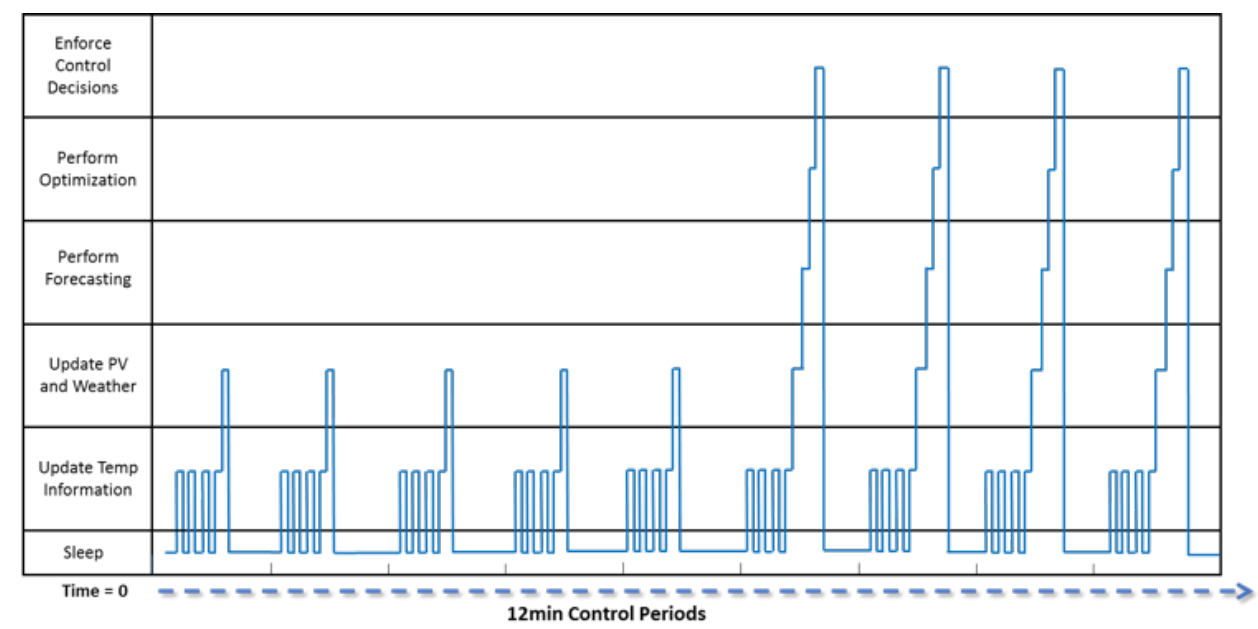

Figure A-87. Timing diagram for the MPC controller.

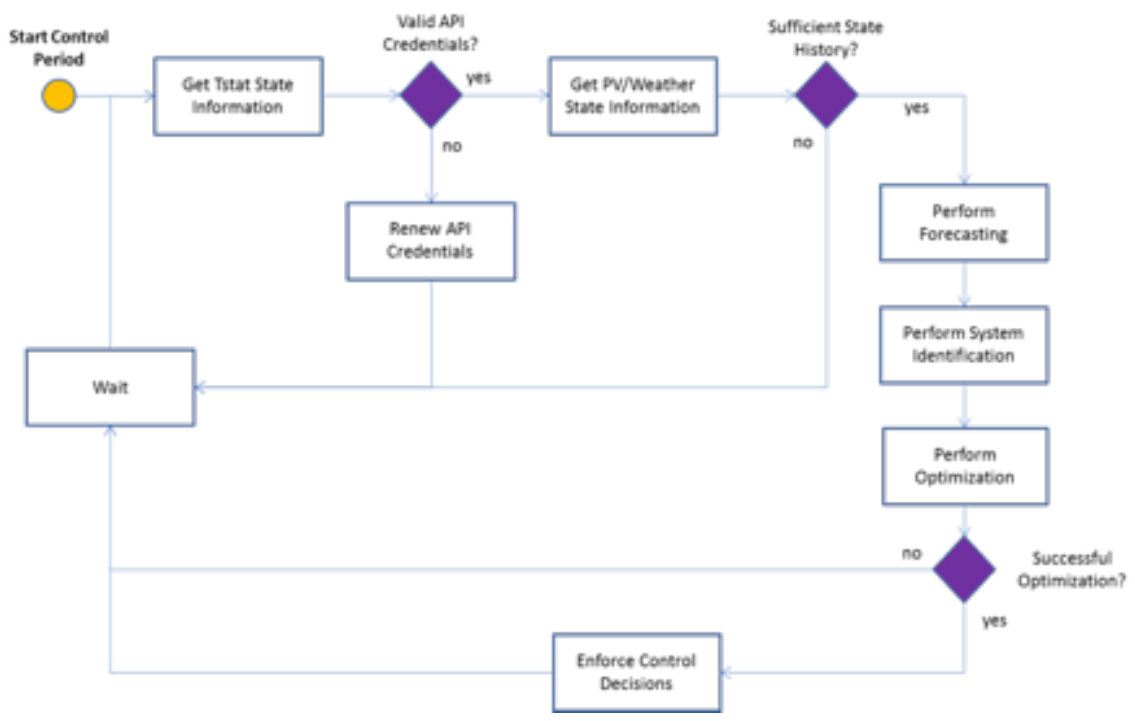

Figure A-88. Control workflow for the MPC control.

\section{A.9 UNCERTAINTY ANALYSIS}

\section{A.9.1 Uncertainty Analysis for Building Power Consumption}

This section assesses the variability in the building power consumption as a result of weather, occupancy variables, and various sources of uncertainty in model parameters and model fidelity. The team started from an EnergyPlus model of the building, which was subjected to an uncertainty analysis (UA). The UA is based on the well-established propagation of sampled values of all uncertain variables.

Building on prior efforts, the Georgia Tech team established an Uncertainty and Risk Analysis Workbench (GURA-W) [153-154] to add rigor to the process based on generic preestablished uncertainty ranges of EnergyPlus model parameters. Moreover, the workbench allows the modeler to add model form uncertainties that represent discrepancies in various EnergyPlus modules, define time series for weather and occupancy variables, and conduct a sensitivity analysis for selected outcomes of the simulation. Figure A-89 shows the architecture of GURA-W. 


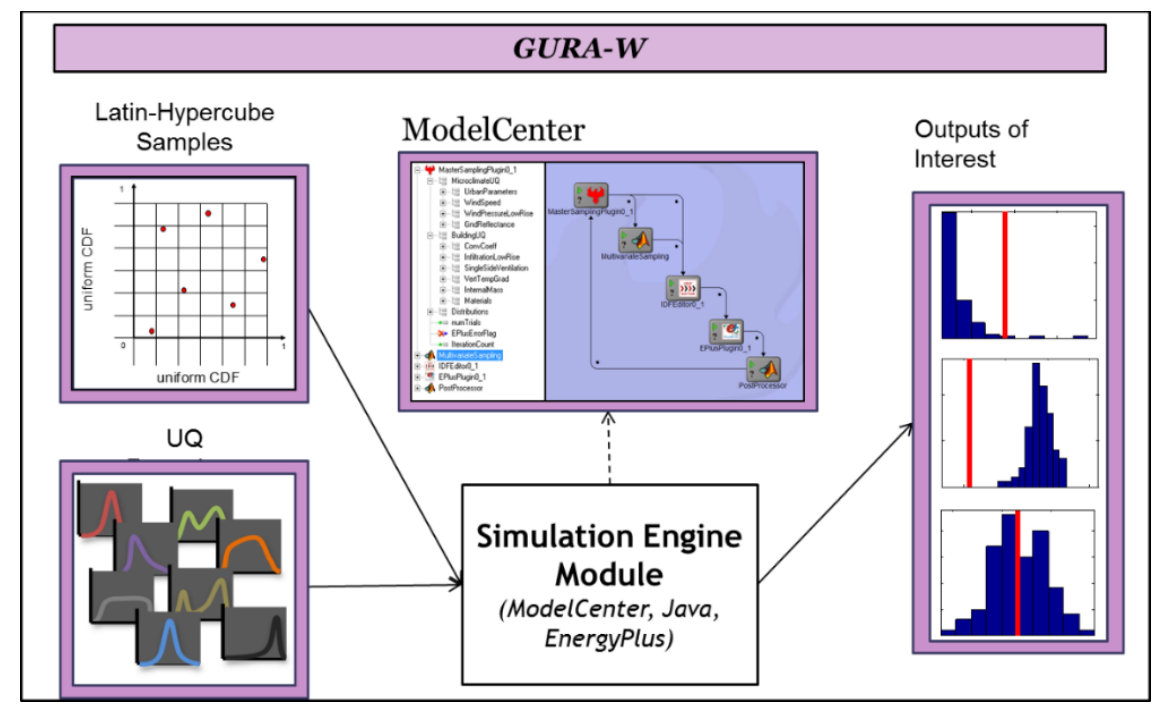

Figure A-89. The architecture of the UA tool (GURA-W).

One main deliverable of the EFRI project is the uncertainty quantification (UQ) repository that contains quantified and validated uncertainty distributions, as well as stochastic models for weather and occupancy variables. Additionally, model form uncertainties are seeded within the EnergyPlus model because internal stochastic processes representing model discrepancies. For this reason, a customized (i.e., recompiled) version of EnergyPlus is embedded in GURA-W. Examples of model form uncertainties are the urban heat island effect, local wind speed and pressure, temperature stratification, and convective heat transfer. This approach was used on the DOE small office reference building to inspect the variability of power consumption patterns, particularly to verify the effect on the dominant frequencies in the patterns. Figure A-90 illustrates the power duration curve in which the shaded band shows the effect of uncertainties. Figure A-91 illustrates the sensitivity analysis results. The team observed that the uncertainty in power peak is about $20-30 \%$ and that the variability in occupancy, lighting, and appliance scenarios has the largest influence. Additionally, only a small set of building parameters has a relevant impact on resulting power uncertainty.

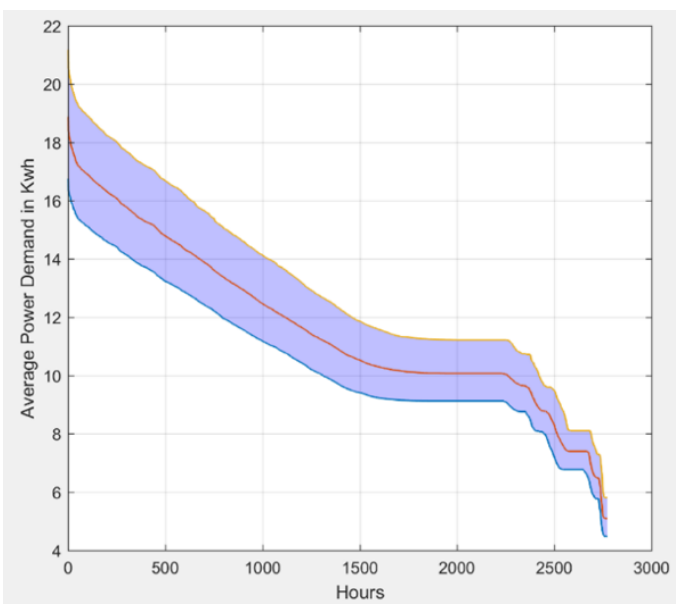

Figure A-90. Power duration curve for a building power consumption.

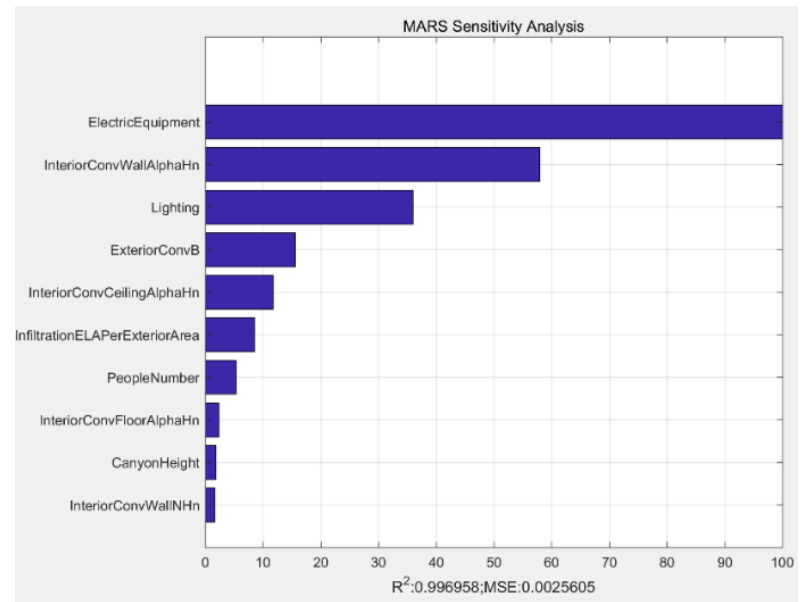

Figure A-91. Sensitivity analysis results for a building power consumption. 
Moreover, the frequencies in the power consumption were also investigated. Figure A-92 illustrates a typical outcome.
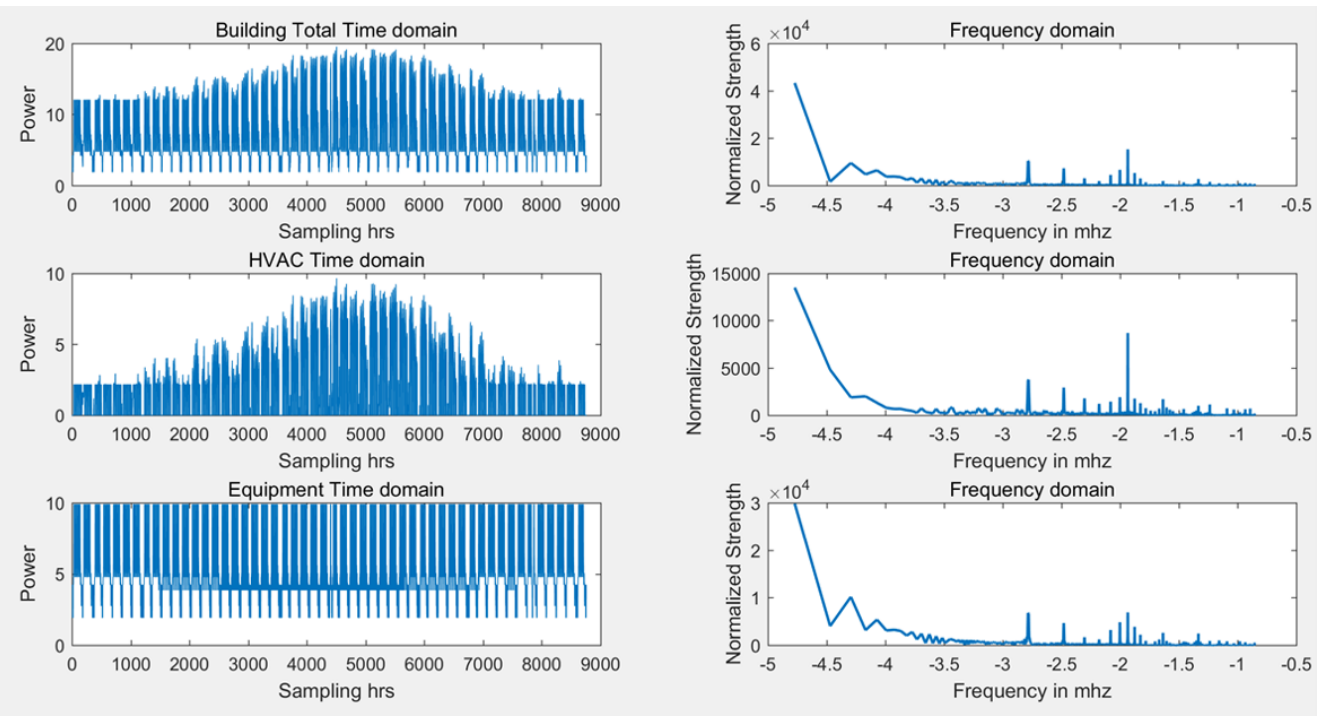

Figure A-92. Frequency-domain analysis of frequencies for a building power consumption.

When subjected to an UA for the mentioned sources of uncertainty in the small office reference building, a relatively small impact on the frequency distribution was found, as illustrated in Figure A-93 for summer.
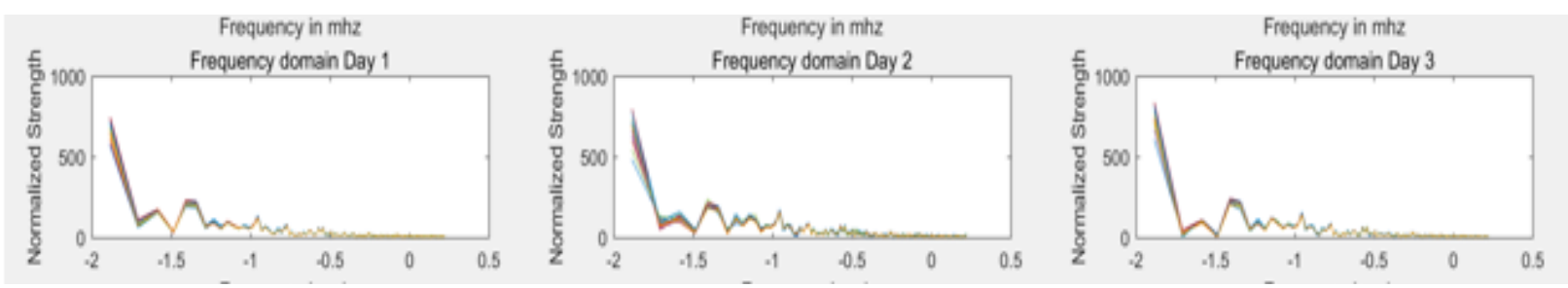

Figure A-93. Sensitivity analysis results for the frequencies in the building power consumption.

The results in this section show that a dynamic simulation with embedded uncertainty adequately reveals the magnitude of power consumption variability in the demand side.

\section{A.9.2 The Analysis of Zonal Controllability in HVAC System Power Interruption}

The developed controllers in this project schedule buildings HVAC systems based on the fact that they can operate without HVAC service for a specific length of time due to their slow dynamics (i.e., time constants). It will cause the space temperature to exceed the set point and increase gradually. The speed at which this and the recovery afterwards occur depends on the characteristics of building. To capture this "controllability" phenomenon, a controllability measure was introduced. From a high-level point of view, it is reasonable to assume that a measure of building controllability is related to three main building characteristics: the building active thermal mass, the daily load profile, and the capacity of the HVAC system.

As a measure of building controllability, defining three performance indicators is appropriate:

1) Unmet space temperature period, which is defined as the time that: 


$$
T_{i}>T_{\text {set }}+1 K
$$

2) Maximum temperature difference between $T_{i}$ and $T_{\text {set }}$.

3) The 12th $(1 \mathrm{~h})$ highest value over time steps of the temperature difference between $T_{i}$ and $T_{\text {set }}$.

In this study, all input parameters are normalized per $1 \mathrm{~m}^{2}$ of floor area. The cooling system is controlled based on on/off strategy to reflect the central control logic. The governing equation is shown as follows:

$$
\begin{gathered}
C \frac{d T_{i}}{d t}=Q_{\text {load }}-\alpha Q_{\text {cool }}, \\
Q_{\text {cool }}=Q_{\text {cap }} * \eta *\left(T_{\text {space }}-T_{\text {set }}\right) \| Q_{\text {cool }} \leq Q_{\text {cap }},
\end{gathered}
$$

where $C\left[\mathrm{~J} / \mathrm{m}^{2}\right]$ is the equivalent heat capacity of building mass; $Q_{\text {load }}$ is the total building heat gain, which is generated for a typical office building in a given climate zone in EnergyPlus; $Q_{\text {cool }}$ is the cooling supplied by the HVAC system; $\eta$ is the HVAC response coefficient; and $\alpha$ indicates the availability of the HVAC system, which is 0 during a power interruption period. t $Q_{c o o l}$ is capped between zero and the HVAC cooling capacity, which is known based on the design or nameplate information.

The hypothesis is that the value of the controllability measure generated with this simple model with the values for a given building substituted in the model can be used as a suitable indicator to benchmark the controllability of any building. Figure A-94 shows the correlation between the value of the postulated measure with the value of the measure based on EnergyPlus results. If the value of the measure meets the benchmark, then it will be deemed a good candidate for the developed control approach.

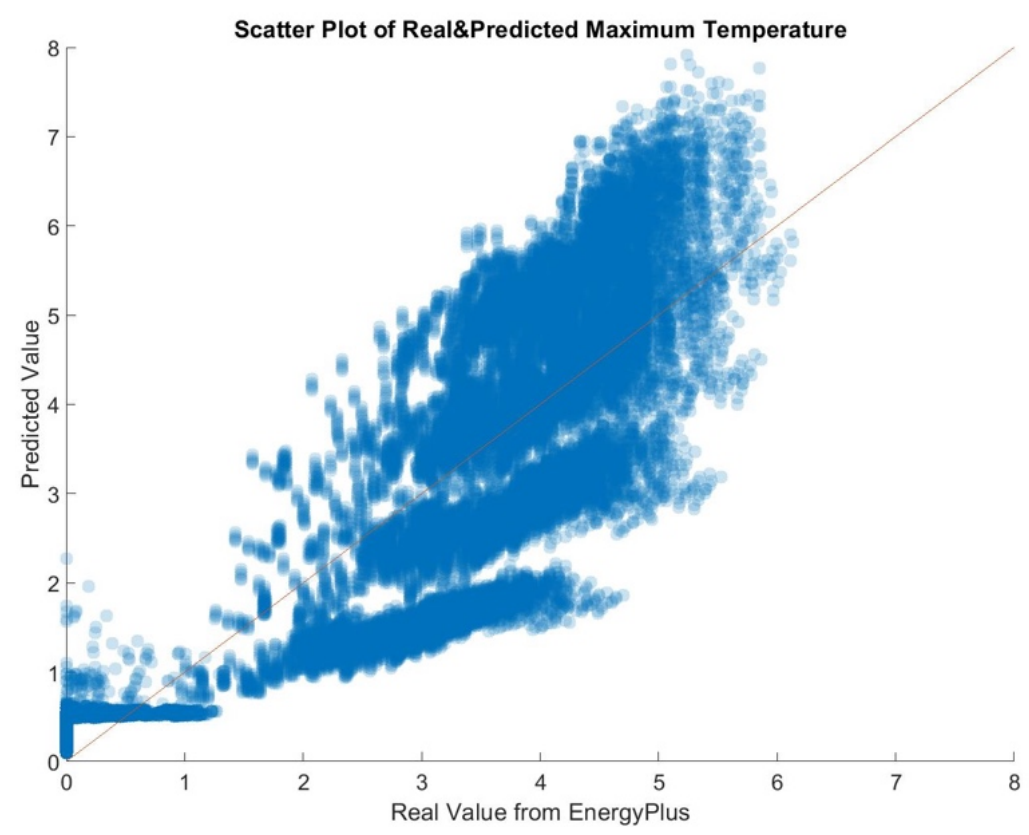

Figure A-94. Value of the postulated measure with the value of the measure based on EnergyPlus results.

Based on the hypothesis made in the previous section, four key variables were chosen as the input: HVAC capacity, building active thermal mass, HVAC nonavailable starting point, and HVAC nonavailable duration. Each input variable was categorized into five levels, and the simulation period was the 64 
weekdays from June to August. This led to roughly 40,000 data points. The supervised learning method, k-nearest neighbors (KNN), was used to train a model to compute the controllability measure. To benchmark a given building, a threshold that indicates the minimum necessary controllability is defined. The inputs are the four key variables, and the output is the controllability measure.

Two experiments were conducted with minimum unavailability duration as $15 \mathrm{~min}$ and $30 \mathrm{~min}$, respectively. The experimental setup and results are shown in Tables A-8 through A-10.

Table A-8. Experimental setup for the controllability test.

\begin{tabular}{|c|c|c|c|c|}
\hline Level & $\begin{array}{c}\text { Cooling capacity } \\
(\%)\end{array}$ & Thermal mass level & Nonavailable hour & Nonavailable duration \\
\hline $\mathrm{I}$ & 90 & Very light & 5:00 & $0 \min (0 \mathrm{~min})$ \\
\hline II & 100 & Light & $8: 00$ & $15 \min (30 \mathrm{~min})$ \\
\hline III & 110 & Medium & 11:00 & $30 \min (60 \mathrm{~min})$ \\
\hline IV & 120 & Heavy & $14: 00$ & $45 \min (90 \mathrm{~min})$ \\
\hline $\mathrm{V}$ & 130 & Very heavy & $17: 00$ & $1 \mathrm{~h}(2 \mathrm{~h})$ \\
\hline \multicolumn{2}{|c|}{ Threshold of $15 \mathrm{~min}$} & \multicolumn{2}{|l|}{ Unmet hour $<0.5 \mathrm{~h}$} & $\max <$ Tset $+4 \mathrm{~K}$ \\
\hline \multicolumn{2}{|c|}{ Threshold of $30 \mathrm{~min}$} & Unmet hour $<1.5 \mathrm{~h}$ & \multicolumn{2}{|c|}{ Temperature $\max <$ Tset $+4 \mathrm{~K}$} \\
\hline
\end{tabular}

Table A-9. Prediction accuracy for $15 \mathrm{~min}$.

\begin{tabular}{|c|c|c|}
\hline \multicolumn{3}{|c|}{ Prediction accuracy in 15 min experiment } \\
\hline & Predicted good & Predicted bad \\
\hline Actual good & 6430 & 125 \\
\hline Actual bad & 220 & 5100 \\
\hline Accuracy & \multicolumn{2}{|c|}{$97 \%$} \\
\hline
\end{tabular}

Table A-10. Prediction accuracy for 30 min.

\begin{tabular}{|c|c|c|}
\hline \multicolumn{3}{|c|}{ Prediction accuracy in 30 min experiment } \\
\hline & Predicted good & Predicted bad \\
\hline Actual good & 6853 & 624 \\
\hline Actual bad & 386 & 4012 \\
\hline Accuracy & \multicolumn{2}{|c|}{$91 \%$} \\
\hline
\end{tabular}

The results show that the KNN model can accurately predict the controllability of the building. However, the supervised learning method requires an EnergyPlus model and simulation for every combination of the four building parameters. This requires a large modeling effort and expensive simulations. Therefore, the team attempted to develop a reduced building model (i.e., a one-node RC model to reduce the modeling and simulation effort). Although the RC model was not an accurate representation of each building, the generation of a controllability measure with the RC model could still be sufficiently accurate. To test this assumption, the team conducted an experiment with the RC model, generating many data samples by choosing different combinations of HVAC capacity, building active thermal mass, and weather conditions, which were represented by a load shape function, in combination with different interruption scenarios (i.e., different HVAC nonavailability starting points and durations). The result based on this experiment indicate the control potential of a building under any given load, HVAC capacity, and mass availability operating in different HVAC nonavailability scenarios. The results show that the first-order RC model can predict the controllability correctly in $80 \%$ of all cases. In future work, the experiment could be repeated with a higher order RC model to see whether the $80 \%$ accuracy could be increased significantly. 


\section{A.9.3 Uncertainty Quantification of RC Model}

To develop a fast-reacting building energy model for predictive control, the team developed an RC model calibrated on simulation results generated with EnergyPlus. The DOE reference building EnergyPlus model of the small office was used for generating simulated results that mimic the actual behavior of the building under a designated control. The team applied 212 uncertainty factors to the simulation with GURA-W to represent all possible realizations and usages of a building. The number of unmet hours calculated with the RC model was compared with the unmet hours generated with EnergyPlus. The comparison was used to examine the RC model's accuracy under various hypothetical power availability scenarios.

The following two RC model usage variants were examined in the development of the RC model:

- the RC model used to predict room temperature in 30 min intervals (one time step) and

- the RC model used to predict room temperature for $3 \mathrm{~h}$ (six time steps).

The following assumptions were made.

- All input variables used in the RC model training are measurable during the application to a real building.

- The relationship between thermal cooling energy and HVAC electricity consumption is known.

In a future stage of the development, these assumptions will be further analyzed to characterize the uncertainties in these assumptions and propagate them in the use of the RC model to verify suitability.

The RC model is developed as follows:

$$
C * \frac{d T}{d t}=\frac{1}{R} *\left(T_{a}-T_{i}\right)+\alpha_{1} * Q_{S o l}+\alpha_{2} * Q_{\text {Int }}+Q_{\text {cool }},
$$

where:

$T_{a}$ is ambient temperature,

$T_{i}$ is room air temperature at the target zone,

$Q_{\text {Int }}$ is hourly reference value for internal heat gain (no uncertainty included),

$Q_{\text {hvac }}$ is cooling rate for the target zone, and

$Q_{S o l}$ is global solar radiation on the façades the target zone.

In an actual field application, these RC model parameters are calibrated in real time with temperature and power data that are continuously collected from the actual building. These measurements must be augmented by predictions of weather and usage scenarios. Together with the imprecise conversion from power to thermal cooling, it will introduce uncertainties that must be considered in the calibration of the parameters. As mentioned, this will be studied in the next stage of the project.

In the current stage, a UA with EnergyPlus was used to create a synthetic training dataset for the RC model calibration. The UA (i.e., the propagation of the regular set of "design uncertainties") serves a special purpose that is very different from the real-time calibration setting. In those settings, the actual building is available and observable; hence, the set of uncertainties and their ranges are much smaller. The team used the "full" UA to reflect the design situation in which the building was only designed and not yet realized. In such cases, the RC model analysis is done to reveal the utility of the RC model approach during a not yet constructed facility (i.e., in the design of the overall control system). For this 
purpose, the team used the EnergyPlus+GURA-W simulation outcomes as the training data for the RC model parameters $\left(R, C, \alpha_{1}, \alpha_{2}\right)$. Given the uncertainties in the simulation outcomes, this will result in distributions of the value of these parameters. The results show that in some cases, the RC model is not trainable or shows incorrect prediction for extreme outliers (5-20\%). The RC model must be trained on the last available inputs within a rolling window of $72 \mathrm{~h}$ to avoid too much impact of scenario uncertainty (SU) (e.g., set point schedule). The analysis is done for a five-zone simulation in which the RC model is trained for each zone separately with the temperature data of that zone. Obviously, the accuracy of the RC model prediction of unmet hours varies per zone, as shown in Table A-11. Table A-11 shows the average $3 \mathrm{~h}$ prediction accuracy of the RC model over all uncertainty runs in the test period. The RC model generally exaggerates the number of predicted unmet hours.

Table A-11. The average prediction accuracy of the RC model over all uncertainty runs.

\begin{tabular}{|c|c|c|c|c|c|}
\hline Zone & Unmet hour & $\begin{array}{c}\text { Correct } \\
\text { prediction }\end{array}$ & $\begin{array}{c}\text { Wrong } \\
\text { prediction }\end{array}$ & $\begin{array}{c}\text { Correct } \\
\text { percentage (\%) }\end{array}$ & $\begin{array}{c}\text { Wrong } \\
\text { percentage (\%) }\end{array}$ \\
\hline South & 104.5 & 82 & 69 & 78.5 & 66.0 \\
\hline East & 103.5 & 94 & 50 & 90.8 & 48.3 \\
\hline North & 108 & 83 & 72 & 76.9 & 66.7 \\
\hline West & 103 & 7805 & 70 & 76.2 & 68.0 \\
\hline Center & 109 & 108.5 & 20 & 99.5 & 18.3 \\
\hline
\end{tabular}

In the building thermal model in Eq. (A-98), the cooling amount from HVAC system is usually assumed to be known and treated as an input variable for both model training and prediction. The $Q_{\text {cool }}$ in Eq. (A-98) represents the cooling amount that goes to the room. There are some weaknesses of using the term $Q_{\text {cool, }}$ including the following.

1. The cooling delivered by the HVAC system to a zone cannot be measured directly. The best alternative is to express it as $Q_{e l e}{ }^{*} \mathrm{COP}$, which is only correct for the thermal cooling delivered to the whole building.

2. The COP is not constant over time, hence the calculated cooling amount is inaccurate.

3. The amount of cooling generated by the HVAC system is distributed to the separate zones of a building in a way that is dependent on the distribution layout and control. At best, the actual $Q_{\text {cool }}$ to a zone of the building can be approximated given the attributes and controls of the distribution system.

To obtain the exact $Q_{\text {cool }}$ in model training and predictive control, an auto feedback loop was applied in the RC model to mimic the real control logic in the HVAC system. This leads to:

$$
C \frac{d T}{d t}=\frac{1}{R}\left(T_{a}-T_{i}\right)+\alpha_{1} Q_{\text {Sol }}+\alpha_{2} Q_{\text {Int }}+\eta\left(T_{i}-T_{\text {set }}+\theta\right) .
$$

In the feedback loop, $T_{i}$ is the room air temperature, $T_{\text {set }}$ is the set point temperature, $\theta$ is the setback temperature, and $\eta$ is the conversion factor to $Q_{\text {cool }} . \theta$ and $\eta$ are the parameters calibrated from model training. The model training is accomplished with a calibration over a rolling window of $72 \mathrm{~h}$, which means that the parameters in the RC model are recalibrated every hour with the newest $72 \mathrm{~h}$ data points. 
This is currently conducted with simulation (EnergyPlus)-generated "sensor data" because as the real sensor data are not available. The temperature set point is used as an input variable. Compared with $\mathrm{Q}_{\text {cool, }}$, the temperature set point over time is much easier to obtain and is usually kept on a constant schedule in office buildings. In this form, the cooling amount calculated from the auto feedback loop can capture the required cooling amount that in this simple one-node $\mathrm{RC}$ model goes to the active thermal mass.

Based on the same training and prediction setup introduced previously, the newer model with autofeedback loop was compared with the original one. In Table A-12, there is $20-50 \%$ reduction of the mean absolute error (MAE) prediction by implementing the autofeedback loop. The distribution of the prediction error after a certain time is shown in Figure A-95.

Table A-12. Temperature prediction accuracy.

\begin{tabular}{|c|l|r|r|r|r|r|r|}
\hline \multicolumn{6}{|c|}{ Temperature Prediction Mean Absolute Error (1-6 steps) $(30-180 \mathrm{~min})$ (C) } \\
\hline \multirow{3}{*}{ Orignal } & Steps & 1 & 2 & 3 & 4 & 5 & 6 \\
& All Hours & 0.40 & 0.71 & 0.77 & 0.76 & 0.85 & 0.92 \\
& Office Hour & 0.82 & 1.39 & 1.40 & 1.30 & 1.41 & 1.47 \\
\hline \multirow{3}{*}{ Auto Feedback } & Steps & 1 & 2 & 3 & 4 & 5 & 6 \\
& All Hours & 0.27 & 0.43 & 0.53 & 0.60 & 0.66 & 0.72 \\
\hline & Office Hour & 0.43 & 0.64 & 0.71 & 0.74 & 0.79 & 0.83
\end{tabular}
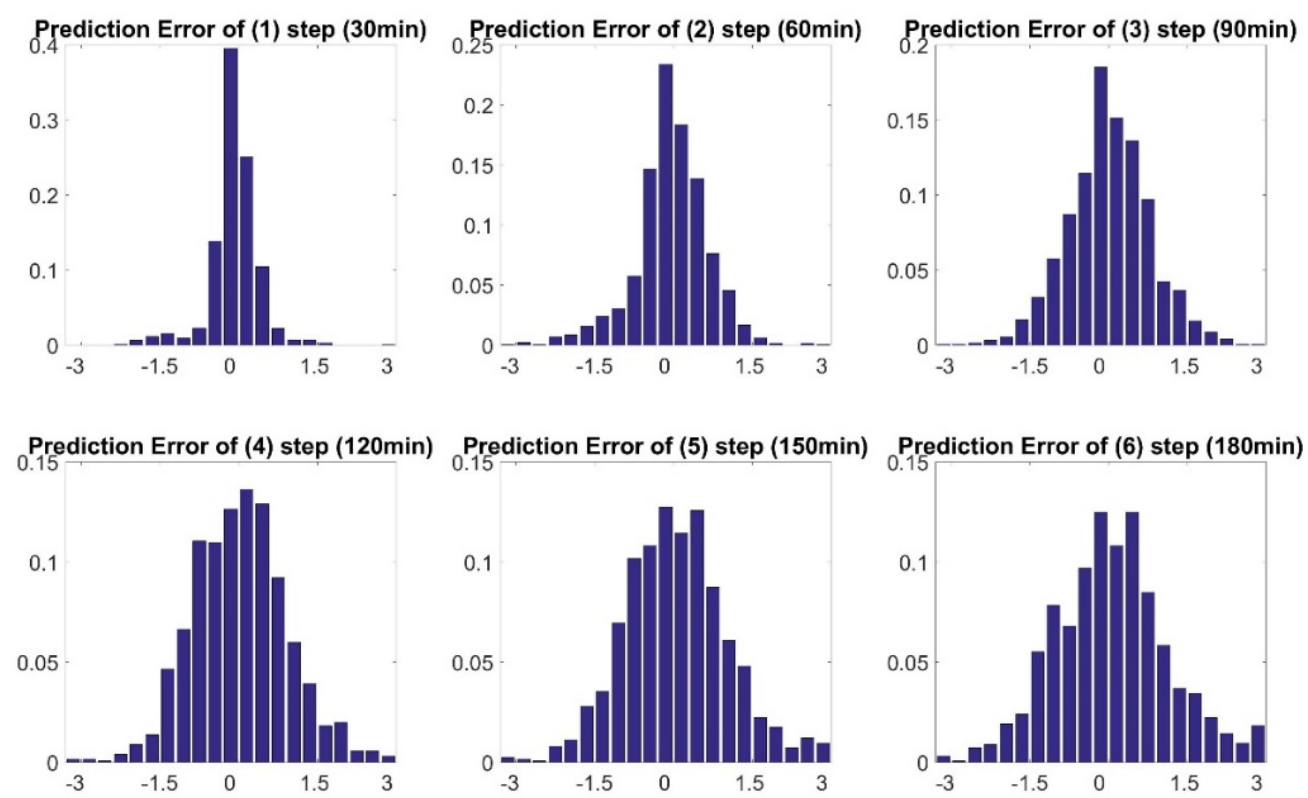

Figure A-95. Distribution of the prediction error after a certain time.

The simple one-node RC model is obviously unable to represent the building dynamics very accurately. This discrepancy can be expressed as $R C$ model form uncertainty (MFU). The Georgia Tech team set out to quantify MFU based on a comparison between EnergyPlus outcomes with embedded uncertainty and $\mathrm{RC}$ model outcomes. This was done for one specific zone of the small commercial DOE reference building. The team assumed that this setup would lead to a quantified MFU of the RC model that is indicative of the MFU magnitude for a large class of commercial buildings. Further work must confirm this assumption, but first it must be established whether the MFU represents a significant source of error in using the RC model to predict building behavior. This MFU quantification was done for the one-node 
RC model introduced previously and calibrated according to the procedure explained there. The process is shown in Figure A-96.

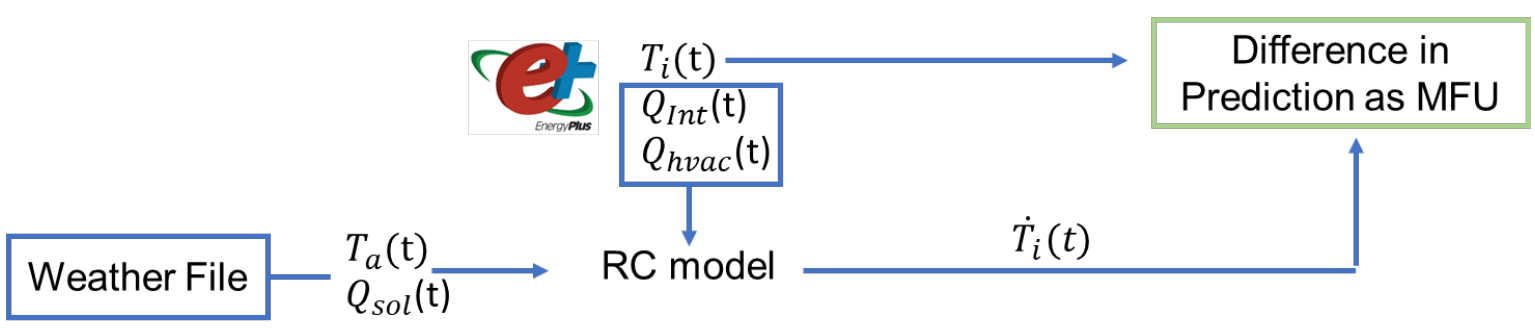

Figure A-96. RC building model calibration using the MFU quantification process.

The team performed the MFU quantification for each EnergyPlus simulation sample outcome generated by the GURA-W UA of the building. MFU was thus quantified based on the results of 360 EnergyPlus uncertainty samples, resulting in 360 outcomes for July with all typical-212, in this case - uncertainty parameters performed in GURA-W. The RC model was trained in the rolling window of 3 days and then used to produce the temperature prediction for the following period. The MFU was then quantified by using a statistical training technique that expresses the error between the two models. The quantified discrepancy can now be added to the $\mathrm{RC}$ model equation as a $\delta$, leading to:

$$
C \frac{d T}{d t}=\frac{1}{R}\left(T_{a}-T_{i}\right)+\alpha_{1} Q_{\text {Sol }}+\alpha_{2} Q_{\text {Int }}+\eta\left(T_{i}-T_{\text {set }}+\theta\right)+\boldsymbol{\delta} .
$$

The MFU quantification in the RC model shows a $-0.3^{\circ} \mathrm{C}$ mean error with a $1.2^{\circ} \mathrm{C}$ standard deviation over 144 time steps, as shown in Figure A-97. As expected, because of the effect of the autofeedback loop, the mean prediction error was maintained at a relatively small value and did not increase over time; the standard deviation increased rapidly in the first 10 time steps before it converges to a constant value.
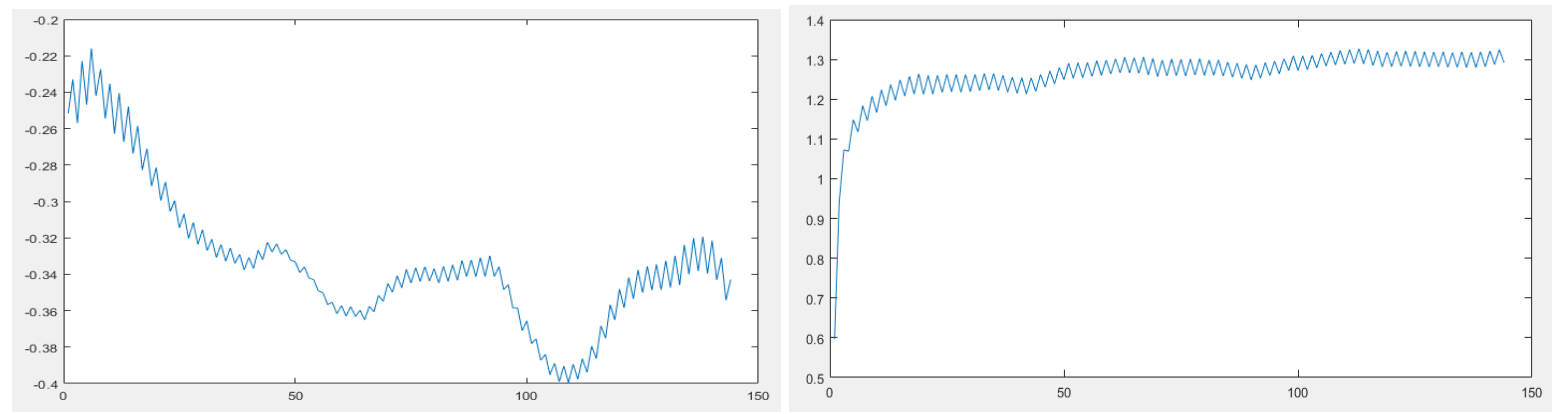

Figure A-97. The MFU quantification in the RC model over 144 time steps.

Several statistical methods were used in the quantification, including regression methods, Gaussian process, Brownian motion, and time series models. The outcomes show that: (1) the regression methods are inappropriate for quantifying MFU because the correlations between the parameters and inputs of the $\mathrm{RC}$ model and the prediction error are insignificant, (2) the autofeedback loop bounds the variation of the prediction error to a form of Brownian motion, and (3) the most effective method proves to be a time series model with a lag-2 autocorrelation based on the current training data (i.e., the difference between the two model outcomes).

SU captures the uncertainty of input variables related to internal scenarios (e.g., usage) and external scenarios (e.g., weather) to which the building is subjected. These can often not be known exactly, which is the case for the varying internal heat gain $\left(Q_{i n t}\right)$ and cannot be measured directly. In practice, at best, the 
mean people and plug load density and schedule can be derived from the building type and zone function from which a mean profile of the internal heat gain can be derived. However, the dynamics around the mean are usually unknown. The outdoor air temperature $\left(T_{a}\right)$ and global horizontal solar radiation intensity $\left(Q_{\text {sol }}\right)$ are obtained from weather forecasts or a local prediction model. However, they are not exact, and the resulting prediction errors must be introduced into the RC model. For the RC model calibration, the measured value of $T_{a}$ and $Q_{s o l}$ can be used, but in the consequent use of the RC model, only the forecast weather can be used for prediction. For $Q_{i n t}$, there is no measured value available, so an approximation of $Q_{i n t}$ based on average profile plus uncertain dynamic fluctuations should be used for training and prediction. To study the influence of SU on the RC prediction accuracy, a 100 run RC model training and prediction was performed with uncertainty in the weather and scenario. Table A-13 shows the MAE with SU over all runs; the SU only led to a 5-15\% MAE increase.

Table A-13. Temperature prediction accuracy.

\begin{tabular}{|l|l|r|r|r|r|r|r|}
\multicolumn{7}{|c|}{ Temperature Prediction Mean Absolute Error (1-6 steps) (30-180min) (C) } \\
\multirow{3}{*}{ Without SU } & Steps & 1 & 2 & 3 & 4 & 5 & 6 \\
& All Hours & 0.27 & 0.43 & 0.53 & 0.60 & 0.66 & 0.72 \\
& Office Hour & 0.43 & 0.64 & 0.71 & 0.74 & 0.79 & 0.83 \\
\hline \multirow{3}{*}{ with All SU } & Steps & 1 & 2 & 3 & 4 & 5 & 6 \\
& All Hours & 0.29 & 0.46 & 0.56 & 0.64 & 0.71 & 0.79 \\
& Office Hour & 0.45 & 0.67 & 0.74 & 0.80 & 0.85 & 0.91
\end{tabular}

Figure A-98 shows the band of temperature prediction with SU compared with the actual temperature. Along with the lengthening of the prediction period, the error in the temperature prediction increased significantly.
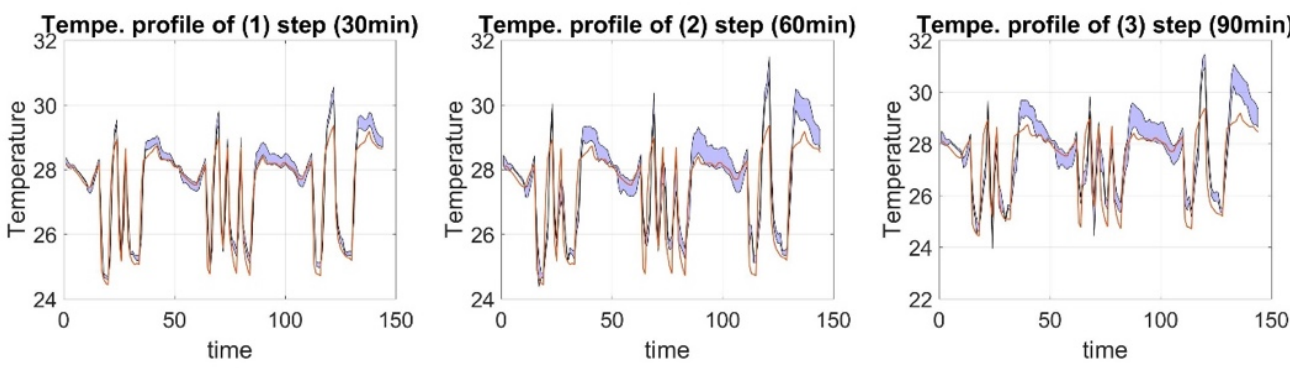

Tempe. profile of (4) step (120min)
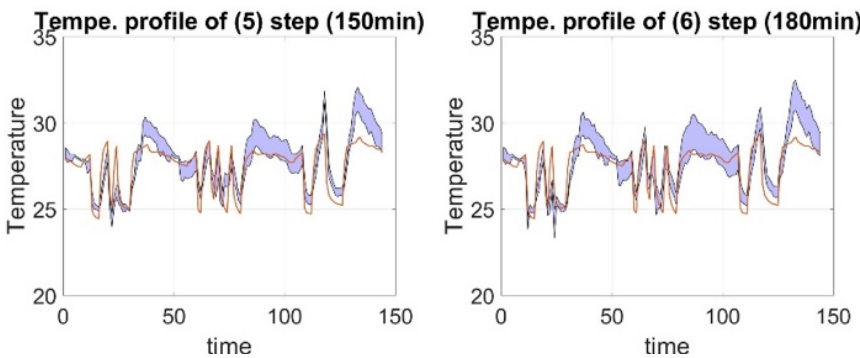

Figure A-98. The temperature prediction with SU compared with the actual temperature.

From this analysis, the error introduced by SU is relatively small compared with the error introduced by MFU. The relative dominance of the role of MFU indicates that it makes sense to try to decrease the magnitude of MFU, which could be achieved by an RC model improvement (e.g., by a two-node RC model). 


\section{A.9.4 Integrating the Building Energy Model Inside the Overall System Architecture}

The work in this quarter focused on integrating the building energy model inside the total system architecture, as proposed by ORNL (Figure A-99). The team used this architecture to develop in vitro simulation testing for the embedded system that will be deployed later for real-time decision-making.

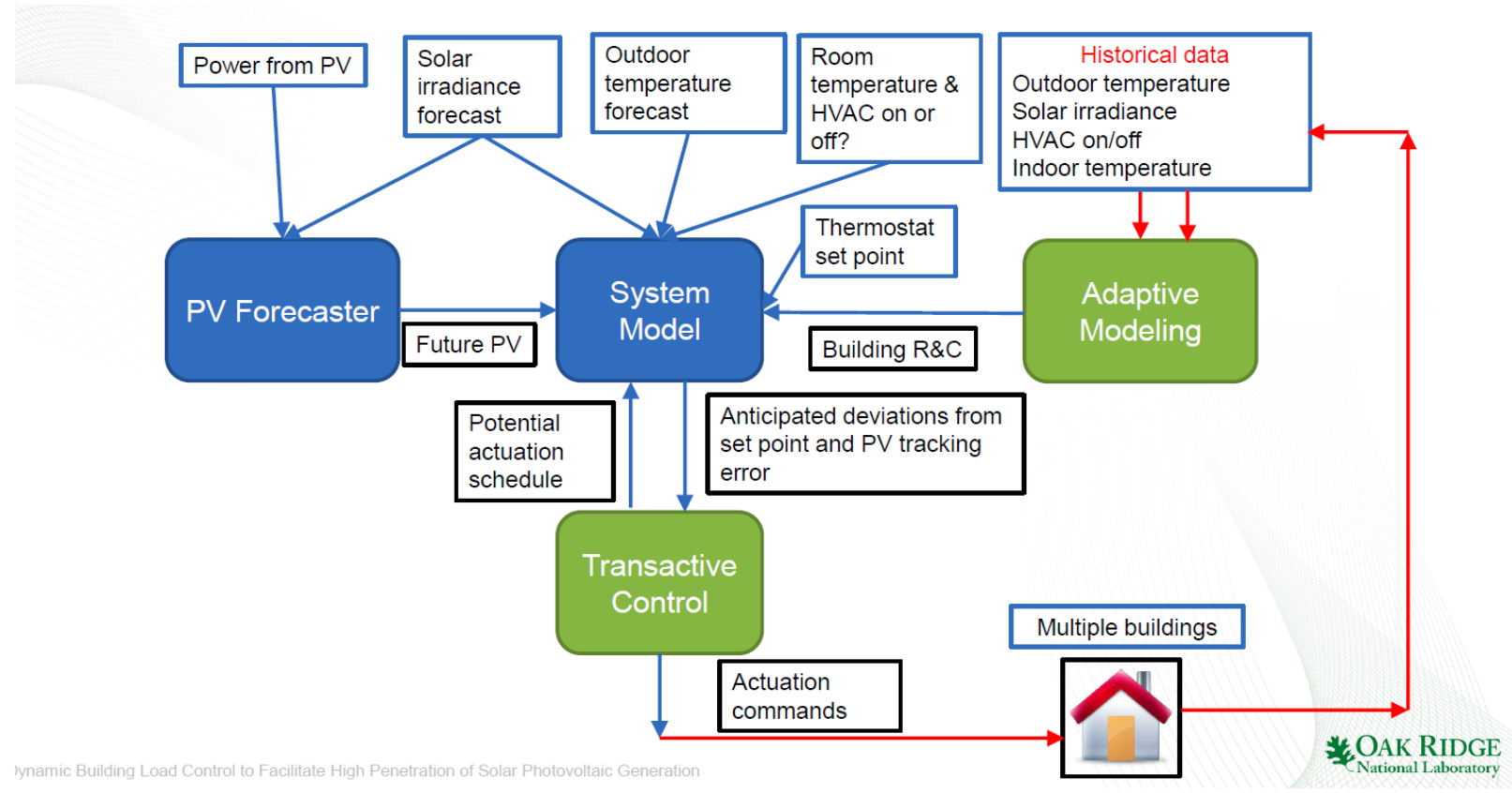

Figure A-99. Control information flow.

The main purpose of the testing environment is to simulate variable building behavior and test the prediction and control algorithms (Figure A-100). 


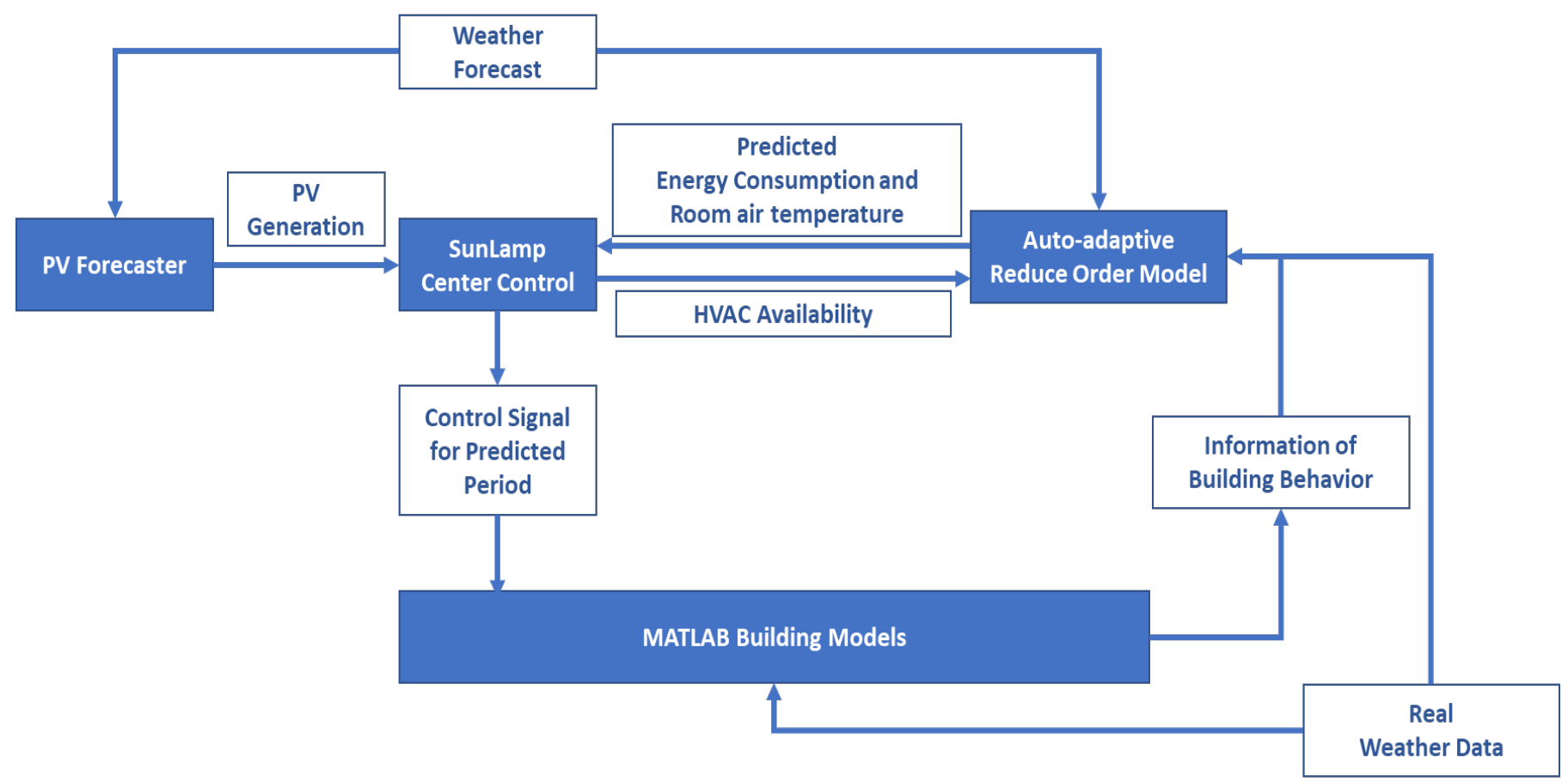

Figure A-100. In vitro testing in simulation test bank.

There are four modules in the test bank. These modules exchange the information recurrently in every iteration. In the current study, $1 \mathrm{~h}$ is used as the interval for one iteration. The interval for the on/off control signals for each HVAC unit is 10 min (i.e., six control steps per hour).

The modules are detailed as follows.

1) Building Module in MATLAB

A building module was developed in MATLAB to mimic real building behavior in the test bank. It runs 10 small office buildings with five zones in each building.

The MATLAB building module is a high-order finite element model that captures the heat transfer process in the buildings. In this model, each building component is represented by a node, and the thermal interactions between them form a network between nodes. The approach is described in detail in Augenbroe et al. [155].

Variability over buildings and zones comes from different geometry and internal gain features. Each zone contains a unitary cooling unit with modulated control that responds to the thermal load. The availability of the cooling units is controlled by the center control. The MATLAB model takes real weather data as input and provides the information of building behavior for training an auto-adaptive reduced order model for each zone. Building behavior includes zone temperatures over time, the mean profiles of zonal internal heat gains, and HVAC energy consumption profiles. At the end of each iteration, the MATLAB building model receives the control signals provided by the center control and simulates the resulting building behavior. 


\section{2) Auto-Adaptive Reduced-Order Module}

The auto-adaptive reduce order module is a fast-reacting component in the test bank for predicting zone temperature and energy consumption. It is autotrained from the information of building behavior and real weather data. Based on the work reported in the previous quarter, a two-node RC model is proved to have the highest performance for balancing prediction accuracy and model complexity. The formulation of the two-node RC model is shown as follows:

$$
\left[\begin{array}{ccc}
C_{i} & 0 & 0 \\
0 & C_{w} & 0 \\
0 & 0 & 0
\end{array}\right] *\left[\begin{array}{c}
\dot{T}_{i} \\
T_{w} \\
\dot{T}_{a}
\end{array}\right]=\left[\begin{array}{ccc}
-\frac{1}{R_{i}} & \frac{1}{R_{i}} & 0 \\
\frac{1}{R_{i}} & -\left(\frac{1}{R_{i}}+\frac{1}{R_{w}}\right) & \frac{1}{R_{w}} \\
0 & \frac{1}{R_{w}} & -\frac{1}{R_{w}}
\end{array}\right] *\left[\begin{array}{c}
T_{i} \\
T_{w} \\
T_{a}
\end{array}\right]+\left[\begin{array}{c}
\alpha_{1 i} \\
\alpha_{1 w} \\
0
\end{array}\right] * Q_{s o l}+\left[\begin{array}{c}
\alpha_{2 i} \\
0 \\
0
\end{array}\right] * Q_{i n t}-\left[\begin{array}{ccc}
\eta & 0 & 0 \\
0 & 0 & 0 \\
0 & 0 & 0
\end{array}\right] *\left(\left[\begin{array}{c}
T_{i} \\
T_{w} \\
T_{a}
\end{array}\right]-\left[\begin{array}{c}
T_{\text {set }} \\
0 \\
0
\end{array}\right]+\left[\begin{array}{c}
\Theta \\
0 \\
0
\end{array}\right]\right)
$$

This two-node RC model is retrained every hour by using the training data from the last 3 day moving window. Because all 50 zones have different behavior, there are 50 two-node RC models trained in every time step with the building behavior generated by the simulation. After training, each RC model is used to predict the room air temperature and energy consumption based on the weather forecast and predicted internal gains with or without the running HVAC unit, as dictated by the center control.

\section{3) PV Forecast Module}

The team at ORNL provides this model for the prediction of hourly PV generation based on the solar radiation forecast.

\section{4) Center Control}

The center control performs the optimization to match the HVAC consumption with PV generation while maintaining a suitable zone comfort. The objective function is shown as follows:

$$
\arg \min _{u_{i, k}} \sum_{k=1}^{N_{p}}\left\{Q\left(\sum_{i=1}^{N_{s}} f\left(u_{i, k}\right)-P_{p v, k}\right)^{2}+R \sum_{i=1}^{N_{s}} \varepsilon_{i}^{2}\right\}
$$

where:

$i \quad$ is the zone identity (from 1 to 50 ).

$k \quad$ is the time steps to reach the prediction horizon (for now assumed to be from 1 to 36 , which is $10 \mathrm{~min}$ to $6 \mathrm{~h}$ ).

$u_{i, k} \quad$ are the control sequences for each HVAC unit. The control options include forced on, forced off, and no forced control.

$f\left(u_{i, k}\right)$ is HVAC energy consumption of each zone at each step.

$P_{p v, k} \quad$ is the PV generation at each step.

$\varepsilon_{i} \quad$ is the temperature deviation from the original set point.

$Q \& R \quad$ are weighting factors.

Because of the linear formulation of the two-node RC mode, this multi-objective optimization will be realized by quadratic programming to minimize the run time. 